UNIVERSIDADE DE SÃO PAULO

INSTITUTO DE FÍSICA

\title{
Ensembles de matrizes aleatórias normais: projeção, comportamento assintótico e universalidade dos autovalores.
}

\author{
Alexei Magalhães Veneziani \\ Orientador: Prof. Dr. Domingos Humberto Urbano Marchetti \\ Tese de Doutorado \\ submetida ao Instituto de Física \\ da Universidade de São Paulo \\ para a obtenção do título de \\ Doutor em Ciências.
}

Banca Examinadora:

Prof. Dr. Domingos Humberto Urbano Marchetti (IFUSP)

Prof. Dr. João Carlos Alves Barata (IFUSP)

Prof. Dr. Nestor Felipe Caticha Alfonso (IFUSP)

Prof. Dr. Luiz Renato Gonçalves Fontes (IMEUSP)

Profa. Dra. Maria Eulalia Vares (CBPF)

São Paulo

2008 


\section{FICHA CATALOGRÁFICA \\ Preparada pelo Serviço de Biblioteca e Informação do Instituto de Física da Universidade de São Paulo}

Veneziani, Alexei Magalhães

Ensembles de matrizes aleatórias normais: projeção, comportamento assintótico e universalidade dos autovalores - São Paulo, 2008.

Tese (Doutorado) - Universidade de São Paulo. Instituto de Física, Depto. de Física Geral

Orientador: Prof. Dr. Domingos Humberto Urbano Marchetti

Área de Concentração: Física

Unitermos: 1. Matrizes Aleatórias Normais;

2. Distribuição dos autovalores; 3 . Núcleo Integral e Reprodutor; 4. expansão assintótica; 5. Universalidade. 

Dedico esta tese aos meus pais Cesar Luiz Veneziani e Roseli Pinto de Magalhães, à minha avó Margarida Assumpção Veneziani e a Mariana Pojar. 


\section{Agradecimentos}

Ao meu orientador Domingos Humberto Urbano Marchetti, pela paciência, serenidade, atenção, excelente orientação e sobre tudo pelo grande exemplo de pesquisador que é. Foi uma honra ser seu aluno e sempre será um privilégio poder trabalhar contigo.

Aos professores João Carlos Alves Barata, Walter Felipe Wreszinski, José Fernando Perez, Nestor Felipe Caticha Alfonso, Vera Bohomoletz Henriques, Leonardo Fernandes Guide e Roberto da Silva por terem contribuído significativamente para minha formação acadêmica e à professora Carla Goldman pela simpatia.

Aos professores e amigos Max Welcman e Tiago Pereira por terem revisado minha tese.

Aos colegas Flávio Vieira, Evandro Freire, Silas Carvalho, Áttila Leaes, Danilo Liarte, Andre Barato, Renato Germano, Henrique Guide e a muitos outros que de alguma forma contribuíram tanto para minha formação acadêmica quanto para o desenvolvimento deste trabalho.

Aos amigos do bairro onde cresci Tiago, Felipe e Mairo Paes, Eduardo Takeuchi, Tiago Zoia e Fabiano Ferreira, amigos do Objetivo Carlos Brasil, Marcelo Mauziner, Danilo Policastro, Pedro Ross, Osvaldo Peixoto, Fábio Luz e tantos outros que proporcionaram horas inesquecíveis de lazer.

Aos funcionários do Departamento de Física Geral do Instituto de Física da USP, que forneceram um suporte básico para a realização desta tese.

À Fundação de Amparo à Pesquisa do Estado de São Paulo (FAPESP), pelo fundamental apoio financeiro. 


\section{Resumo}

Uma matriz $A_{N}$ de ordem $N$ é normal se e somente se comuta com sua adjunta. Nesta tese investigamos a estatística dos autovalores (no plano complexo) de ensembles de matrizes aleatórias normais quando a ordem $N$ destas tende a infinito. A função distribuição de probabilidade no espaço das matrizes normais atribui, como na mecânica estatística, um peso de Boltzmann $e^{-N F\left(A_{N}\right)}$ a cada realização $A_{N}$ destas matrizes, onde $F$ é uma função a valores reais invariante por transformações unitárias. Realizando uma mudança de variáveis (das variáveis de entrada para as variáveis espectrais), escrevemos a distribuição marginal conjunta dos autovalores $\left\{z_{i}\right\}_{i=1}^{N}$, bem como a função de $n$-pontos correspondente a vários ensembles, como o determinante de um núcleo integral associado.

A partir deste formalismo bem estabelecido na literatura, apresentaremos nesta tese dois tipos de resultados:

Primeiramente, explorando a semelhança da distribuição conjunta dos autovalores a um problema variacional sobre as medidas de equilíbrio eletrostático de cargas sujeitas a um potencial externo $V: \mathbb{C} \rightarrow \mathbb{R}$ (escolhendo $F\left(A_{N}\right) \equiv \sum_{i=1}^{N} V\left(z_{i}\right)$ ), podemos aplicar a teoria de potenciais logarítmicos para obter a única medida de equilíbrio coincidente com a função de 1-ponto destes ensembles. Com base nesta teoria, propomos nesta tese um método de interpolação analítica capaz de projetar a medida de equilíbrio dos ensembles normais em medidas de equilíbrio dos ensembles hermitianos e unitários correspondentes. Ilustramos o procedimento com várias aplicações.

O segundo tipo de resultados utiliza o método de ponto de sela ao núcleo integral da família de ensembles de matrizes normais com potenciais $V_{\alpha}(z)=|z|^{\alpha}, z \in \mathbb{C}$ e $\left.\alpha \in\right] 0, \infty[$. Analogamente ao que foi demonstrado em ensembles hermitianos por Deift, estabelecemos por intermédio desta expansão um conceito similar de universalidade para esta família, fazendo uso de mapas conformes e a teoria de espaços de Segal-Bargmann. Sobre o sentido de universalidade definido por G. Oas, mostramos que a afirmação de universalidade neste sentido por este autor é incorreta quando a cauda desta probabilidade é levada em conta. 


\section{Abstract}

A matrix $A_{N}$ of order $N$ is normal if and only if it commutes with its adjoint. In the present thesis we investigate the eigenvalues statistics (in the complex plane) of ensembles of normal random matrices when their order $N$ tends to infinite. The probability distribution function in the space of normal matrices attributes, as in statistical mechanics, a Boltzmann weight $e^{-N F\left(A_{N}\right)}$ at each matrix realization $A_{N}$, where $F$ is a real-valued function invariant by unitary transformations. By performing a change of variables (from entry variables to spectral variables) we write the marginal joint distribution of eigenvalues $\left\{z_{i}\right\}_{i=1}^{N}$, as well as the $n$-points functions corresponding to several ensembles, as the determinant of an associated integral kernel.

From this formalism well-established in the literature, we shall present in this thesis two types of results:

Firstly, exploiting the similarity of joint distribution of eigenvalues to a variational problem on electrostatic equilibrium measures of charges subjected to an external potential $V: \mathbb{C} \rightarrow \mathbb{R}$ (by choosing $F\left(A_{N}\right) \equiv \sum_{i=1}^{N} V\left(z_{i}\right)$ ), we can apply the theory of logarithmic potentials to obtain the unique equilibrium measure coinciding with the 1-point function of these ensembles. Based on this theory, we propose in this thesis a method of analytical interpolation capable of projecting the equilibrium measure of normal ensembles in equilibrium measures of corresponding Hermitian and unitary ensembles. We give several applications of this procedure.

The second type of results utilizes the saddle point method applied to integral kernel of a family of normal matrix ensembles with potentials $V_{\alpha}(z)=|z|^{\alpha}, z \in \mathbb{C}$ and $\left.\alpha \in\right] 0, \infty[$. Similarly to what has been shown in hermitian ensembles by Deift, we established by mean of this expansion a similar concept of universality for this family, making use of conformal maps and theory of Segal-Bargmann space. Concerning the universality defined by G. Oas, we show that the universality claimed by this author is incorrect when the tail of this probability is taking into account. 


\section{Índice}

1 Introdução $\quad 10$

1.1 Motivações e heurística dos resultados . . . . . . . . . . . . . . . . 10

1.2 Ensembles de matrizes aleatórias . . . . . . . . . . . . . . . . . . . 12

1.2.1 Ensemble de Ginibre . . . . . . . . . . . . . . . . . . . 12

1.2.2 Ensemble Circular . . . . . . . . . . . . . . . . . . 15

1.2.3 Ensemble unitário hermitiano . . . . . . . . . . . . . . . . 16

1.2.4 Ensemble unitário normal . . . . . . . . . . . . . . . . . 17

1.3 Problema de balayage . . . . . . . . . . . . . . . . . . . . 24

1.4 Resumo dos resultados obtidos e roteiro de leitura . . . . . . . . . . . . 25

1.4.1 Relação entre ensembles de matrizes aleatórias . . . . . . . . . . . 25

1.4.2 Núcleo integral de alguns ensembles normais . . . . . . . . . . . . . 29

1.4.3 Universalidade para o ensemble unitário normal . . . . . . . . . . . 31

2 Medidas de equilíbrio e balayage $\quad 38$

2.1 Resultados preliminares conhecidos . . . . . . . . . . . . . . . . 38

2.2 Fórmula explícita para medida de balayage . . . . . . . . . . . . . . . . . . 42

2.3 Ensemble de Dyson e unitário normal circularmente simétrico . . . . . . . 44

2.4 Potenciais de Elbau-Felder . . . . . . . . . . . . . . . . . . . . . . . 47

2.5 Interpolação entre os ensembles de Ginibre-Girko e o de Wigner . . . . . . 48

2.6 Outras relações entre os ensembles normal e hermitiano: Projeção simples (medida marginal $) \ldots \ldots \ldots \ldots \ldots$

$3 \quad$ Núcleo integral para alguns tipos de potenciais $\quad 56$

3.1 Introdução . . . . . . . . . . . . . . . . . . . 56

3.1 Polinômios ortogonais . . . . . . . . . . . . 56

3.1 .2 Núcleo Integral . . . . . . . . . . . . . . . . . . . 57

3.1.3 Função de correlação de n-pontos . . . . . . . . . . . . . . . . 62 
3.2 Potenciais radialmente simétricos . . . . . . . . . . . . . . 63

3.2.1 Potenciais do tipo $V_{\alpha}(z)=|z|^{\alpha} \ldots \ldots \ldots$. . . . . . 64

4 Universalidade para o ensemble unitário normal 88

4.1 Universalidade para o ensemble unitário hermitiano . . . . . . . . . . 88

4.2 Escala para o ensemble unitário normal . . . . . . . . . . . . . . . . 91

4.3 Limite do núcleo integral escalado . . . . . . . . . . . . . . . . . 93

4.3.1 Probabilidade de um disco aberto na vizinhança da origem não conter autovalores . . . . . . . . . . . . . . . . 93

4.3.2 Probabilidade escalada de um disco aberto não conter autovalores . 98

4.4 Universalidade Conforme . . . . . . . . . . . . . . . . . . . . . . . . . . 99

4.5 Universalidade de G. Oas . . . . . . . . . . . . . . . . . . . . . . . . 101

5 Conclusões $\quad 112$

A Ensemble unitário normal $\quad 114$

A.1 Álgebra linear e cálculo diferencial no plano complexo. . . . . . . . . . . . 114

A.2 Jacobiano de $\varphi \ldots \ldots \ldots \ldots \ldots$

B Prova do Teorema 40 com hipóteses restritas $\quad 124$

B.1 Prova do item a . . . . . . . . . . . . . . . . . . . . . 125

B.2 Prova do item b . . . . . . . . . . . . . . . . . . 126

B.3 Prova do item c . . . . . . . . . . . . . . . . . . . . . . . 128

B.3.1 Existência . . . . . . . . . . . . . . . . . . . . 128

B.3.2 Unicidade. . . . . . . . . . . . . . . . . . . . . . 132

B.4 Prova do item d . . . . . . . . . . . . . . . . . . 138

B.5 Prova do item e . . . . . . . . . . . . . . . . . . . 139

C Prova dos Teoremas citados no Capítulo $2 \quad 140$

$\begin{array}{ll}\text { D Cálculo de algumas medidas de equilíbrio } & 147\end{array}$

D.1 Potenciais Radialmente simétricos . . . . . . . . . . . . . . . . . . . . 147

D.2 Ensemble de Dyson no formalismo da Medida de Equilíbrio . . . . . . . . . 149

D.3 Potenciais do tipo $V_{H}(x)=\gamma_{\alpha}|x|^{\alpha} \ldots \ldots \ldots \ldots$. . . . . . . . . . . . .

E Ensemble normal de Ginibre-Girko 156

E.1 Comportamento assintótico. . . . . . . . . . . . . . . . . . . . . . . 159 



\section{Capítulo 1}

\section{Introdução}

\subsection{Motivações e heurística dos resultados}

A investigação sobre a distribuição dos autovalores de matrizes aleatórias teve início na década de 50 com uma série de trabalhos de Wigner [1] motivados por uma aplicação em Física Nuclear. O desenvolvimento subseqüente deveu-se principalmente a Porter [2], Dyson [3], [4] e Mehta [5] e, desde então, a variedade e quantidade de trabalhos em matrizes aleatórias, bem como suas aplicações, cresceram vertiginosamente. Entre as áreas do conhecimento que possuem aplicações de teoria de matrizes aleatórias citamos a conjectura de Riemann, teoria quântica de campos, sistemas integráveis, estatística, sistemas desordenados, gravitação quântica em duas dimensões, caos clássico e quântico. Para uma revisão sobre estes diversos assuntos onde a teoria de matrizes aleatórias pode ser empregada, citamos [6]. Citamos as notas de aula de Percy Deift [7] para contextualizarmos nossa linha de pesquisa em matrizes aleatórias.

Wigner supunha que o fenômeno de captura de nêutrons em núcleos complexos poderia ser descrito somente pela estatística de seus níveis energéticos. Segundo esta hipótese, a descrição detalhada dos níveis excitados do núcleo, além de ser inacessível, não é intrinsecamente relevante para caracterização do fenômeno, mas sim as leis que descrevem o comportamento estatístico dos níveis energéticos. Assim, inspirado na maneira com que a Mecânica Estatística aborda as grandezas macroscópicas em equilíbrio termodinâmico, Wigner propôs descrever os níveis de energia de um núcleo complexo pelos autovalores de um ensemble de matrizes aleatórias, com entradas escolhidas independentemente segundo uma distribuição Gaussiana, porém satisfazendo vínculos atribuídos a simetrias do sistema. 
Em 1965, Ginibre [8] estudou matrizes aleatórias cujas entradas (elementos de matriz) são variáveis aleatórias complexas independentes sem qualquer vínculo e com distribuição gaussiana. Ginibre constatou que a distribuição marginal conjunta dos autovalores podia ser obtida por uma mudança de coordenadas que desacoplava as variáveis dos autovalores das demais variáveis associadas aos autovetores. O jacobiano desta mudança de coordenadas gera um termo de interação logarítmica entre os autovalores, já observado por Dyson nos ensembles de matrizes unitárias, e a normalização da distribuição marginal conjunta destes pode então ser interpretado como sendo a função de partição de um sistema de $N$ cargas de uma única espécie no plano interagindo via um potencial coulombiano (repulsivo, devido às cargas serem de mesmo sinal) e um potencial externo gerado por um fundo (background) contínuo e uniforme de cargas de sinal oposto de forma a estabelecer neutralidade. O potencial externo é devido às entradas das matrizes serem escolhidas independentemente e igualmente distribuídas com distribuição gaussiana.

Segundo esta interpretação, Monvel, Pastur e M. Shcherbina [9] sugerem em sua abordagem para matrizes simétricas que a temperatura neste sistema é proporcional a $N^{-1}$ e, portanto, quando tomamos o limite termodinâmico $(N \rightarrow \infty)$ estamos fazendo simultaneamente o limite $T \rightarrow 0$.

Nossas principais motivações para o estudo de matrizes aleatórias normais são as seguintes:

1. Supondo apenas integrabilidade e invariância por transformações unitárias na função $F$ (veja resumo), a distribuição marginal conjunta dos autovalores destes ensembles de matrizes normais possui a mesma interpretação eletrostática enunciada anteriormente.

2. O potencial coulombiano repulsivo em duas dimensões (logarítmico) é consistente com o fato dos autovalores (cargas) estarem no plano complexo; a interpretação eletrostática de ensembles de matrizes hermitianas ou simétricas, por outro lado, pode ser entendida como caso limite de ensembles de matrizes normais;

3. Ensembles de matrizes hermitianas, simétricas e unitárias são ensembles normais, porém com autovalores projetados sobre curvas no plano complexo; chamaremos os ensembles desse tipo de ensembles normais singulares.

4. A noção de universalidade da distribuição dos espaçamentos entre autovalores de ensemble de matrizes hermitianas pode ser estendida para ensembles de matrizes 
normais. Ao invés de espaçamento entre autovalores investigaremos, alternativamente, a probabilidade de não encontrarmos autovalores em discos centrados na origem no plano complexo para uma família de ensembles de matrizes normais com potenciais $V_{\alpha}(z)=|z|^{\alpha}, z \in \mathbb{N}$ e $\left.\alpha \in\right] 0, \infty[$.

Nesta tese concluiremos que a universalidade se verifica na família de ensembles normais mencionada acima de uma forma ligeiramente diferente da universalidade do ensemble unitário hermitiano proposta em [7]. Um argumento heurístico sobre esta diferença pode ser a diferença de dimensão onde os autovalores se encontram, fazendo com que a influência da repulsão entre os autovalores no ensemble hermitiano, confinados na reta real, seja muito mais relevante que no caso normal $\left(V_{\alpha}\right)$ e, além disso, a maior variedade de arranjos estáveis para o equilíbrio dos autovalores no caso normal, por estarem no plano complexo.

\subsection{Ensembles de matrizes aleatórias}

A teoria de matrizes aleatórias estuda os ensembles de matrizes $M_{N}$ de ordem $N$, cujas entradas (elementos $\left(M_{N}\right)_{i, j}$ da matriz $\left.M_{N}\right)$ são variáveis aleatórias escolhidos de acordo com uma distribuição de probabilidade.

Estamos interessados na distribuição dos autovalores destas matrizes, induzida pela distribuição de suas entradas, quando a ordem $N$ destas tende a infinito. É conveniente, portanto, escalarmos as entradas da matriz, pois geralmente o módulo dos autovalores cresce com a ordem desta (com exceção do ensemble circular definido a seguir). A escala adotada deve ser tal que os autovalores permaneçam num domínio limitado quando $N \rightarrow$ $\infty$.

Estes ensembles dependem, a princípio, das simetrias que a matriz $M_{N}$ possui, do corpo ao qual as entradas pertencem e do tipo de distribuição de probabilidade das entradas. A seguir daremos exemplos de ensembles de matrizes aleatórias devidamente escalados.

\subsubsection{Ensemble de Ginibre}

Notação 1 Denotaremos um número complexo $z \in \mathbb{C}$ por

$$
z=z^{R}+i z^{I}=r e^{i \theta}
$$


com $i^{2}=-1, \operatorname{Re} z=z^{R}, \operatorname{Im} z=z^{I} \in \mathbb{R}, r \in \mathbb{R}^{+} \cup\{0\}$ e $\left.\left.\theta \in\right]-\pi, \pi\right]$ e o complexo conjugado de z por

$$
\bar{z}=z^{R}-i z^{I}=r e^{-i \theta} .
$$

O traço de uma matriz quadrada A de ordem $N$ como sendo a soma dos elementos de sua diagonal principal:

$$
\operatorname{Tr}[A]=\sum_{i=1}^{N} A_{i i}
$$

A matriz transposta de $A$ será denotada por $A^{t}$ e por $A^{*}$ a matriz adjunta da matriz $A$ :

$$
A_{i, j}^{*}=\bar{A}_{j, i} \text { ou } A^{*}=\bar{A}^{t}
$$

Se $\mu$ é uma medida de Borel positiva tal que

$$
\int_{\mathbb{K}} d \mu=1
$$

onde $\mathbb{K}=\mathbb{C}$ ou $\mathbb{R}$, então chamaremos $\mu$ de medida de probabilidade.

Definição 2 O ensemble de Ginibre é formado pelo conjunto das matrizes complexas $G_{N}$ (sem simetria) com elementos independentes

$$
\left(G_{N}\right)_{i, j}=\frac{g_{i, j}}{\sqrt{N}}
$$

cuja distribuição de probabilidade é dada por

$$
P\left(G_{N}\right) d G_{N}=\frac{1}{Z_{N}} e^{-N \operatorname{Tr}\left[G_{N}^{*} G_{N}\right]} \prod_{1 \leq i, j \leq N}\left(d^{2} G_{N}\right)_{i j}
$$

onde $Z_{N}$ é a normalização de $P$.

Observação 3 Note que de (1.2) obtemos

$$
\begin{aligned}
P\left(G_{N}\right) d G_{N} & =\frac{1}{Z_{N}} e^{-N \sum_{i, j=1}^{N} \frac{\bar{g}_{i, j}}{\sqrt{N}} \frac{g_{i, j}}{\sqrt{N}}} \prod_{1 \leq i, j \leq N} \frac{d^{2} g_{i j}}{N} \\
& =\frac{1}{Z_{N} N^{N}} e^{-\sum_{i, j=1}^{N}\left(\left(g_{i, j}^{R}\right)^{2}+\left(g_{i, j}^{I}\right)^{2}\right)} \prod_{1 \leq i, j \leq N} d g_{i j}^{R} d g_{i j}^{I} \\
& =\frac{1}{Z_{N} N^{N}} \prod_{1 \leq i, j \leq N} e^{-\left(g_{i, j}^{R}\right)^{2}} d g_{i j}^{R} e^{-\left(g_{i, j}^{I}\right)^{2}} d g_{i j}^{I}
\end{aligned}
$$

e, portanto, $\left\{g_{i, j}\right\}_{i, j=1}^{N}$ são variáveis aleatórias complexas estatisticamente independentes (pois a medida de probabilidade das entradas é da forma produto) com distribuição gaussiana. 
Observação 4 O fator $N$ em $e^{-N \operatorname{Tr}\left[G_{N}^{*} G_{N}\right]}$ na expressão (1.2) acima é um fator de escala para garantir que os autovalores permaneçam com módulo finito quando a ordem $N$ da matriz tende a infinito. Neste caso vemos explicitamente que esta escala induz em cada elemento de matriz a escala $\frac{1}{\sqrt{N}}$ em (1.1). Se $\left\{z_{k}\right\}_{k=1}^{N}$ e $r_{N}=\max _{1 \leq k \leq N}\left|z_{k}\right|$ são, respectivamente, os autovalores e o raio espectral (escalado como antes) de $G_{N}$, então

$$
r_{N}=1+O\left(\sqrt{\frac{\log N}{N}}\right)
$$

em probabilidade [10]. Mais precisamente

$$
\lim _{N \rightarrow \infty} P_{N}^{G}\left(\sqrt{4 N \gamma_{N}}\left(r_{N}-1-\sqrt{\frac{\gamma_{N}}{4 N}}\right) \leq x\right)=e^{-e^{-x}}
$$

onde

$$
\gamma_{N}=\log \frac{N}{2 \pi}-2 \log \log N
$$

e $P_{N}^{G}$, a distribuição conjunta dos autovalores escalados $\left\{z_{k}\right\}_{k=1}^{N}$ de $G_{N}$, é dada por

$$
P_{N}^{G}\left(z_{1}, \cdots, z_{N}\right) \prod_{1 \leq i \leq N} d^{2} z_{i}=\frac{1}{Z_{N}^{G}} \mathrm{e}^{-N \sum_{i=1}^{N}\left|z_{i}\right|^{2}} \prod_{1 \leq i<j \leq N}\left|z_{i}-z_{j}\right|^{2} \prod_{1 \leq i \leq N} d^{2} z_{i} .
$$

Ginibre [8] demonstrou pela primeira vez que a distribuição conjunta dos autovalores escalados de $G_{N}$ é de fato dada pela expressão acima. Também que a função correlação de 1 ponto normalizada (interpretada por Ginibre como a densidade dos autovalores):

$$
\rho_{N}^{G}(z)=\frac{1}{N} R_{1}^{G}(z)=\int_{\mathbb{C}^{N-1}} P_{N}^{G}\left(z, z_{2}, \cdots, z_{N}\right) \prod_{2 \leq i \leq N} d^{2} z_{i}
$$

converge em distribuição (quando $N \rightarrow \infty$ ) para a distribuição uniforme no disco unitário do plano complexo:

$$
\lim _{N \rightarrow \infty} \rho_{N}^{G}(z)=\frac{1}{\pi} \chi_{\mathbb{D}}(z)
$$

onde $\chi_{\mathbb{D}}$ é a função indicadora do conjunto

$$
\mathbb{D}=\{z \in \mathbb{C}:|z| \leq 1\}
$$

Bai [11] demonstrou que este resultado permanece válido em um contexto mais geral. Se $A_{N}$ é uma matriz aleatória da forma

$$
\left(A_{N}\right)_{i j}=\frac{a_{i j}}{\sqrt{N}}
$$


com $\left\{a_{i j}\right\}_{i, j=1}^{N}$ variáveis aleatórias igualmente distribuídas e estatisticamente independentes, com distribuição com média zero, variância unitária e sexto momento finito, então a medida empírica dos autovalores de $A_{N}$

$$
d \mu_{N}(z)=N^{-1} \sum_{i=1}^{N} \delta\left(z-z_{i}\right) d^{2} z
$$

converge com probabilidade 1 quando $N \rightarrow \infty$ para a medida uniforme em $\mathbb{D}(1.5)$.

\subsubsection{Ensemble Circular}

Uma matriz $U_{N}$ de ordem $N$ é unitária se e somente se

$$
U_{N}^{-1}=U_{N}^{*} \equiv \bar{U}_{N}^{t}
$$

onde $U_{N}^{-1}$ é a matriz inversa de $U_{N}$.

Notemos que uma matriz complexa possui $2 N^{2}$ variáveis reais independentes e, portanto, uma matriz complexa unitária possui

$$
2 N^{2}-N^{2}=N^{2}
$$

elementos reais independentes devido à equação (1.6).

Definição 5 O ensemble circular é formado pelo conjunto das matrizes $U_{N}$ unitárias cujos elementos independentes são variáveis aleatórias complexas com distribuição de probabilidade invariante por automorfismos, ou seja,

$$
P\left(U_{N}\right) d U_{N}=P\left(\widetilde{U}_{N}\right) d \widetilde{U}_{N}
$$

onde

$$
\widetilde{U}_{N}=S_{N} U_{N} V_{N}
$$

para toda matriz $S_{N}$ e $V_{N}$ unitária e $d U_{N}$ é a medida invariante no grupo das matrizes unitárias de ordem $N$ (medida de Haar).

Podemos notar que os autovalores de $U_{N}$ têm módulo 1 e, portanto, não é necessário escalar suas entradas. Este ensemble também é conhecido como ensemble circular ou simplesmente ensemble de Dyson.

A distribuição conjunta dos autovalores no ensemble circular é

$$
P_{N}^{D}\left(z_{1}, \cdots, z_{N}\right)=\frac{1}{Z_{N}^{D}} \prod_{1 \leq k<j \leq N}\left|e^{i \theta_{k}}-e^{i \theta_{j}}\right|^{2}, z_{k}=e^{i \theta_{k}}, k=1, \cdots, N
$$


e a função correlação de 1 ponto

$$
R_{1}^{D}(z)=N \int_{\partial \mathbb{D}^{N-1}} P_{N}^{D}\left(\theta, \theta_{2}, \cdots, \theta_{N}\right) \prod_{2 \leq i \leq N} d \theta_{i}=\frac{N}{2 \pi}
$$

foram calculadas por Dyson em [3, 4].

Se interpretarmos a função correlação de 1 ponto normalizada como a densidade dos autovalores

$$
\rho_{N}^{D}(z)=\frac{1}{N} R_{1}^{D}(z)=\frac{1}{2 \pi}
$$

então $\rho_{N}^{D}(z)$ converge em distribuição (quando $N \rightarrow \infty$ ) para a medida uniforme na circunferência unitária

$$
\partial \mathbb{D}=\{z \in \mathbb{C}:|z|=1\}
$$

\subsubsection{Ensemble unitário hermitiano}

Uma matriz $N \times N$ complexa $H_{N}$ é chamada de hermitiana se e somente se

$$
H_{N}=H_{N}^{*}
$$

Neste caso, $H_{N}$ possui $N^{2}$ elementos reais independentes, pois a equação (1.8) estabelece $N^{2}$ vínculos:

$$
\left(H_{N}\right)_{i j}=\left(\bar{H}_{N}\right)_{j i} \text { com } 1 \leq i \leq j \leq N .
$$

Além disso, tanto os autovalores quanto os elementos da diagonal principal de uma matriz complexa hermitiana são reais.

Definição 6 O ensemble unitário hermitiano é formado pelo conjunto das matrizes hermitianas cujos elementos independentes $\left\{\left(H_{N}\right)_{i, j}\right\}_{1 \leq i \leq j \leq N}$ são variáveis aleatórias complexas com distribuição de probabilidade invariante por transformações unitárias:

$$
P\left(H_{N}\right) d H_{N}=P\left(\widetilde{H}_{N}\right) d \widetilde{H}_{N}, d H_{N}=\prod_{1 \leq i \leq N}\left(d H_{N}\right)_{i i} \prod_{1 \leq i<j \leq N}\left(d H_{N}^{R}\right)_{i j}\left(d H_{N}^{I}\right)_{i j}
$$

onde

$$
\widetilde{H}_{N}=U_{N}^{*} H_{N} U_{N}
$$

para toda matriz unitária $U_{N}$.

No caso em que

$$
\begin{aligned}
P\left(H_{N}\right) d H_{N} & =\frac{1}{Z_{N}} e^{-\frac{N}{4} \operatorname{Tr}\left[H_{N} H_{N}^{*}\right]} \prod_{1 \leq i \leq j \leq N}\left(d H_{N}\right)_{i j} \\
& =\frac{1}{Z_{N}} \prod_{1 \leq i \leq N} e^{-\frac{N}{4}\left(H_{N}^{2}\right)_{i i}\left(d H_{N}\right)_{i i}} \prod_{1 \leq i<j \leq N} e^{-\frac{N}{2}\left(H_{N}^{R}\right)_{i j}^{2}}\left(d H_{N}^{R}\right)_{i j} e^{-\frac{N}{2}\left(H_{N}^{I}\right)_{i j}^{2}}\left(d H_{N}^{I}\right)_{i j}
\end{aligned}
$$


temos explicitamente que as entradas não diagonais

$$
\left(H_{N}\right)_{i, j}=\frac{h_{i, j}}{\sqrt{N}}
$$

onde $\left\{h_{i, j}\right\}_{1 \leq i<j \leq N}$ são variáveis aleatórias complexas estatisticamente independentes com distribuição gaussiana de média zero e variância unitária. A distribuição conjunta dos autovalores $\left\{\lambda_{i}\right\}_{i=1}^{N}$ deste ensemble é

$$
P_{N}^{W}\left(\lambda_{1}, \cdots, \lambda_{N}\right) \prod_{1 \leq i \leq N} d \lambda_{i}=\frac{1}{Z_{N}^{W}} e^{-N \sum_{i=1}^{N} \frac{\left|\lambda_{i}\right|^{2}}{2}} \prod_{1 \leq i<j \leq N}\left(\lambda_{i}-\lambda_{j}\right)^{2} \prod_{1 \leq i \leq N} d \lambda_{i}
$$

e a função correlação de 1 ponto normalizada

$$
\rho_{N}^{W}(\lambda)=\frac{1}{N} R_{1}^{W}(\lambda)=\int_{\mathbb{R}^{N-1}} P_{N}^{W}\left(\lambda, \lambda_{2}, \cdots, \lambda_{N}\right) \prod_{2 \leq i \leq N} d \lambda_{i}
$$

no limite $N \rightarrow \infty$ converge em distribuição para a lei do semicírculo:

$$
\lim _{N \rightarrow \infty} \rho_{N}^{W}(\lambda)=\frac{1}{2 \pi} \sqrt{4-x^{2}} \chi_{[-2,2]}(x) .
$$

Podemos citar [1] para uma revisão destes resultados.

Observação 7 Bai [12] também estendeu a validade deste resultado. Se $H_{N}$ (como em (1.9)) possuir $\left\{h_{i, j}\right\}_{1 \leq i<j \leq N}$ variáveis aleatórias complexas igualmente distribuídas e estatisticamente independentes, com média zero, variância unitária (não é necessário este requerimento para os elementos da diagonal principal de $\left.H_{N}\right)$, então a medida empírica dos autovalores no limite $N \rightarrow \infty$ converge com probabilidade 1 para a lei do semicírculo (1.10).

\subsubsection{Ensemble unitário normal}

Nesta tese estudamos o ensemble unitário normal bem como sua relação com alguns ensembles unitários normais singulares (ensembles circular e unitário hermitiano). Assim, daremos mais detalhes sobre este ensemble.

Definição 8 Uma matriz $M_{N}$ complexa de ordem $N$ é normal se e somente se existe uma matriz unitária $U_{N}$ tal que

$$
U_{N}^{*} M_{N} U_{N}=\Lambda_{N}
$$

onde

$$
\Lambda_{N}=\left(\begin{array}{ccc}
z_{1} & \cdots & 0 \\
\vdots & \ddots & \vdots \\
0 & \cdots & z_{N}
\end{array}\right)=\operatorname{diag}\left\{z_{1}, \cdots, z_{N}\right\}
$$


é uma matriz diagonal de ordem $N$. Alternativamente, uma matriz $M_{N}$ é normal se e somente se

$$
\left[M_{N}, M_{N}^{*}\right]=M_{N} M_{N}^{*}-M_{N}^{*} M_{N}=0
$$

(veja Seção 5.2 de [13]).

Observação $9 \mathrm{Na}$ equação (1.11), $\Lambda_{N}$ é a matriz dos autovalores de $M_{N}$ e $U_{N}$ como a matriz cujas colunas são os autovetores ortonormais de $M_{N}$. É conveniente ordenar os autovalores e correspondentes autovetores da seguinte forma: Defina $\prec(e \preccurlyeq)$ uma relação ordem total no conjunto dos números complexos definida por

1. $z \prec w$ se $|z|<|w|$

2. $z \prec w$ se $|z|=|w| e \arg (z)<\arg (w)$

3. $z \preccurlyeq w$ se $|z|=|w| e \arg (z) \leq \arg (w)$

onde $|z| e \arg (z)$ são, respectivamente, o módulo e o argumento do número complexo z. Escolha $\Lambda_{N}$ de forma que $\left\{z_{1}, \cdots, z_{N}\right\}$ são ordenados neste sentido. Este ordenamento induz um ordenamento nas colunas de $U_{N}$ (que são os autovetores de $M_{N}$ ) via equação (1.11). Doravante sempre escolheremos $U_{N}$ e $\Lambda_{N}$ da forma mencionada nesta Observação. A escolha de $U_{N}$ não é única, pois

$$
V_{N}=U_{N} \operatorname{diag}\left\{e^{i \theta_{1}}, \cdots, e^{i \theta_{N}}\right\} \text { com }-\pi<\theta_{i} \leq \pi \text { para todo } i=1, \cdots, N
$$

também satisfaz (1.11). Notamos também (por (1.11) e (1.12)) que $\Lambda_{N}$ tem $2 N$ coordenadas reais independentes e $U_{N} \bmod \mathbb{T}^{N}$, onde

$$
\mathbb{T}^{N}=\left\{\left(e^{i \theta_{1}}, \cdots, e^{i \theta_{N}}\right), 0 \leq \theta_{i}<2 \pi\right\}
$$

possui $N^{2}-N$ coordenadas reais independentes. Os autovetores de $U_{N}$ são normalizados e, portanto, definidos a menos de uma fase.

Observação 10 Toda matriz hermitiana é normal. De fato, se $H_{N}$ é uma matriz hermitiana, então

$$
\left[H_{N}, H_{N}^{*}\right]=H_{N} H_{N}-H_{N} H_{N}=0 .
$$

Além disso, toda matriz unitária é normal:

$$
\left[U_{N}, U_{N}^{*}\right]=U_{N} U_{N}^{-1}-U_{N}^{-1} U_{N}=1_{N}-1_{N}=0
$$

Estes fatos fazem com que o ensemble unitário hermitiano e o ensemble circular de Dyson sejam ensembles unitários normais sujeitos a vínculos adicionais. 
Definição 11 O ensemble unitário normal é formado pelo conjunto das matrizes normais cujos elementos independentes são variáveis aleatórias complexas com distribuição de probabilidade invariante por transformações unitárias, ou seja,

$$
P\left(M_{N}\right) d M_{N}=P\left(\widetilde{M}_{N}\right) d \widetilde{M}_{N}, d M_{N}=\prod_{1 \leq i \leq j \leq N}\left(d M_{N}^{R}\right)_{i j}\left(d M_{N}^{I}\right)_{i j}
$$

onde

$$
\widetilde{M}_{N}=U_{N}^{*} M_{N} U_{N}
$$

para toda matriz unitária $U_{N}$.

Trataremos ensembles normais com distribuição de probabilidade da forma

$$
P\left(M_{N}\right) d M_{N}=Z_{N}^{-1} e^{-N F\left(M_{N}\right)} d M_{N}
$$

onde $N$ é o fator de escala, $Z_{N}^{-1}$ é tal que

$$
\int P\left(M_{N}\right) d M_{N}=1
$$

$d M_{N}$ é como em (1.13) e $F$ uma função a valores reais tal que $F$ é invariante por transformações unitárias, ou seja,

$$
F\left(M_{N}\right)=F\left(U_{N} M_{N} U_{N}^{*}\right)
$$

para qualquer matriz $U_{N}$ unitária.

Observação 12 A medida de probabilidade (1.14) é de fato invariante por transformações unitárias devido à (1.15) e

$$
d \widetilde{M}_{N}=d M_{N}
$$

Veja a prova desta identidade na Seção A.2 do Apêndice. Devido à propriedade (1.15) atribuída a $F$, podemos escolher a matriz unitária $U_{N}$ que diagonaliza $M_{N}$ para obtermos

$$
F\left(M_{N}\right)=F\left(U_{N}^{*} M_{N} U_{N}\right)=F\left(\Lambda_{N}\right) \equiv \digamma\left(z_{1}, \cdots, z_{N}\right),
$$

ou seja, $F$ depende apenas dos autovalores de $M_{N}$. Doravante escolheremos $F$ da forma

$$
\digamma\left(z_{1}, \cdots, z_{N}\right)=\sum_{i=1}^{N} V\left(z_{i}\right)
$$

onde $V: \Sigma \subset \mathbb{C} \rightarrow \mathbb{R}$ é uma função (doravante denominada potencial) com certas propriedades, enunciadas no Capítulo 2. 
Seja $U_{N}$ a matriz unitária dos autovetores de $M_{N}$ e consideramos a representação espectral (1.11) como uma mudança de coordenadas $\varphi$, que leva as entradas independentes de $M_{N}$ nas variáveis espectrais

$$
M_{N} \stackrel{\varphi}{\longrightarrow}\left(\Lambda_{N}, U_{N} \bmod \mathbb{T}^{N}\right)
$$

Como o conjunto das matrizes normais com autovalores simples $\left(z_{i} \neq z_{j}\right.$ para $\left.i \neq j\right)$ tem medida de Lebesgue total no espaço $\mathcal{N} \simeq \mathbb{R}^{N^{2}+N}$ das matrizes normais e o ordenamento dos autovalores induz um ordenamento dos autovetores normalizados nas colunas da matriz $U_{N}$, a transformação (1.16) e sua inversa estão bem definidas localmente, de forma que as variáveis espectrais dão origem a uma maneira conveniente de parametrizar localmente as matrizes normais de forma suave. Referimos à Seção 5.3 de [7] para uma construção de $\varphi$ para o ensemble unitário hermitiano. Esta construção pode ser generalizada de maneira direta para o caso do ensemble unitário normal, tendo em vista que:

1. a estrutura dos autovetores em ambos os ensembles é idêntica;

2. a diferença na parte dos autovalores pode ser resolvida aumentando em $N$ a dimensão do espaço das matrizes hermitianas $\mathcal{H} \simeq \mathbb{R}^{N^{2}}$;

3. a questão do ordenamento dos autovalores complexos das matrizes normais é solucionada pela relação de ordem total discutida na Observação 9.

Na Seção A.2 do Apêndice calculamos explicitamente o jacobiano da mudança das $N^{2}+N$ coordenadas independentes $\left\{M_{k l}, \overline{M_{k l}}\right\}_{k \geq l=1}^{N}$ das entradas da matriz normal $M_{N}$ para $2 N$ coordenadas espectrais $\left\{z_{i}, \bar{z}_{i}\right\}_{i=1}^{N}$ que representam os autovalores complexos de $M_{N}$ e as $2 l=N(N-1)$ coordenadas complexas $\left\{p_{i}, \bar{p}_{i}\right\}_{i=1}^{l} \subset U_{N}$ (que estão associadas aos autovetores normalizados de $M_{N}$ ).

Observação 13 A independência das coordenadas $\{z, \bar{z}\}$ utilizado acima é no seguinte sentido: o plano complexo $\mathbb{C}$ coincide com o plano cartesiano $\mathbb{R}^{2}$ pela identificação usual

$$
\mathbb{C} \ni z=x+i y \equiv(x, y) \in \mathbb{R}^{2} .
$$

e, portanto, tem duas estruturas de espaço vetorial: uma como um espaço vetorial de dimensão 2 sobre $\mathbb{R}$ e outra como um espaço vetorial de dimensão 1 sobre $\mathbb{C}$. A partir deste fato, podemos usar as coordenadas

$$
z=x+i y, \bar{z}=x-i y
$$


ao invés das coordenadas $x, y$, as 1-formas

$$
d z=d x+i d y, d \bar{z}=d x-i d y
$$

ao invés das 1-formas $d x, d y$, a 2-forma

$$
d^{2} z=d x \wedge d y=\frac{i}{2} d z \wedge d \bar{z}
$$

(onde $\wedge$ é o produto exterior) e os operadores diferenciais

$$
\frac{\partial}{\partial z}=\frac{1}{2}\left(\frac{\partial}{\partial x}-i \frac{\partial}{\partial y}\right), \frac{\partial}{\partial \bar{z}}=\frac{1}{2}\left(\frac{\partial}{\partial x}+i \frac{\partial}{\partial y}\right)
$$

ao invés dos operadores

$$
\frac{\partial}{\partial x}, \frac{\partial}{\partial y}
$$

Para detalhes veja Seção A.1 do Apêndice.

Realizando a transformação de coordenadas (1.16) em (1.14) obtemos (veja Seção A.2 do Apêndice):

$$
P\left(M_{N}\right) d M_{N}=\widetilde{Z}_{N}^{-1} e^{-N \sum_{i=1}^{N} V\left(z_{i}\right)} J(\varphi) \prod_{1 \leq i \leq N} d^{2} z_{i} \prod_{1 \leq j \leq l} d^{2} p_{k}
$$

onde $d^{2} z, z \in \mathbb{C}$, é a medida de Lebesgue em $\mathbb{C}$ e

$$
J(\varphi)=\left|\Delta_{N}\right|^{2} f(p)
$$

é o Jacobiano da transformação de coordenadas $\varphi$ calculado na Seção A.2 do Apêndice, onde $f(p)$ é uma função positiva que depende apenas das coordenadas $\left\{p_{i}, \bar{p}_{i}\right\}_{i=1}^{l} \mathrm{e}$

$$
\Delta_{N}=\prod_{1 \leq i<j \leq N}\left(z_{i}-z_{j}\right)
$$

é o determinante da matriz de Vandermonde de ordem $N$ formada pelos autovalores $\left\{z_{i}\right\}_{i=1}^{N}$. A distribuição de probabilidade conjunta dos autovalores no ensemble unitário normal é obtida integrando (1.17) com respeito às variáveis $\left\{p_{i}, \bar{p}_{i}\right\}_{i=1}^{l}$ :

$$
P_{N}\left(z_{1}, \cdots, z_{N}\right)=\int_{\mathbb{C}^{l}} P\left(M_{N}\right) \prod_{1 \leq j \leq l} d^{2} p_{j}=\frac{1}{Z_{N}} e^{-N \sum_{i=1}^{N} V\left(z_{i}\right)} \prod_{1 \leq i<j \leq N}\left|z_{i}-z_{j}\right|^{2}
$$

onde

$$
Z_{N}=\int_{z_{1} \prec \cdots \prec z_{N}} e^{-\sum_{i=1}^{N} V\left(z_{i}\right)} \prod_{1 \leq i<j \leq N}\left|z_{i}-z_{j}\right|^{2} \prod_{1 \leq i \leq N} d^{2} z_{i} .
$$

De (1.17) e (1.18) obtemos como conseqüência o seguinte Teorema: 
Teorema 14 Seja $f$ uma função a valores reais positivos tal que $f$ é invariante por transformações unitárias, ou seja, se $M_{N}$ é uma matriz normal, então

$$
f\left(M_{N}\right)=f\left(U_{N} M_{N} U_{N}^{*}\right)
$$

para qualquer matriz unitária $U_{N}$. Então

$$
f\left(M_{N}\right)=g\left(z_{1}, \cdots, z_{N}\right)
$$

é uma função simétrica dos autovalores de $M_{N}$, e

$$
\begin{aligned}
\mathbb{E}[f] & \equiv \int f\left(M_{N}\right) P\left(M_{N}\right) d M_{N} \\
& =\frac{1}{Z_{N}} \int_{z_{1} \prec \cdots \prec z_{N}} g\left(z_{1}, \cdots, z_{N}\right) P_{N}\left(z_{1}, \cdots, z_{N}\right) \prod_{1 \leq i \leq N} d^{2} z_{i} \\
& =\frac{1}{N ! Z_{N}} \int_{\mathbb{C}^{N}} g\left(z_{1}, \cdots, z_{N}\right) P_{N}\left(z_{1}, \cdots, z_{N}\right) \prod_{1 \leq i \leq N} d^{2} z_{i}
\end{aligned}
$$

Prova. A simetria de $f$ decorre diretamente da propriedade (1.19), pois uma permutação da ordem de $\left\{z_{1}, \cdots, z_{N}\right\}$ pode ser implementada por uma conjugação unitária. A prova de (1.20) pode ser encontrada em [7] Seção 5.3 para o caso hermitiano e generalizada trivialmente para o nosso caso fazendo uso da relação de ordem total $\prec$ mencionada na Observação 9.

Observação 15 Comparando (1.18) com (1.3) notamos que a distribuição conjunta dos autovalores do ensemble Ginibre coincide com a distribuição de probabilidade (1.18) quando escolhemos o potencial $V$ da forma

$$
V_{G}(z)=|z|^{2} .
$$

Desta forma definimos o ensemble normal de Ginibre.

\section{Medida de equilíbrio para o ensemble unitário normal.}

Notamos que a expressão (1.18) pode ser escrita como

$$
\begin{aligned}
P_{N}\left(z_{1}, \cdots, z_{N}\right) & =Z_{N}^{-1} \prod_{1 \leq i<j \leq N}\left|z_{i}-z_{j}\right|^{2} e^{-N \sum_{i=1}^{N} V\left(z_{i}\right)} \\
& =Z_{N}^{-1} e^{-\left(\sum_{1 \leq i \neq j \leq N} \log \left|z_{i}-z_{j}\right|^{-1}+N \sum_{i=1}^{N} V\left(z_{i}\right)\right)} \\
& =Z_{N}^{-1} e^{-N^{2} I^{V}\left(\mu_{N}\right)}
\end{aligned}
$$


onde

$$
d \mu_{N}(z)=N^{-1} \sum_{i=1}^{N} \delta\left(z-z_{i}\right) d^{2} z
$$

é a medida empírica dos autovalores de $M_{N}$,

$$
I^{V}(\mu) \equiv \int\left(V(z)+U^{\mu}(z)\right) d \mu(z)
$$

é a "energia eletrostática" associada a $\mu$ (veja Observação a seguir) e

$$
U^{\mu}(z)=\int \log |z-w|^{-1} d \mu(w)
$$

é o potencial logarítmico associado a $\mu$.

Observação 16 Interpretamos a equação (1.25) como sendo o funcional energia de $N$ cargas distribuídas de acordo com a medida empírica $\mu_{N}$ sob a ação de um potencial externo $V$. Assim, a primeira parcela de (1.25) é a energia de interação das $N$ cargas com o potencial $V$ e a segunda parcela é a energia coulombiana de interação entre as $N$ cargas. Notemos que a escala $N$ à frente do potencial $V$ em (1.22) faz com que a energia de interação das cargas com o potencial e a energia de interação entre as cargas fiquem com a mesma ordem em $N$ : este é o argumento físico que justifica a escala dos autovalores de $M_{N}$ para que o suporte da medida empírica (1.24) permaneça em um domínio limitado do plano complexo no limite $N \rightarrow \infty$.

A contribuição dominante a integrais com respeito à (1.23) no limite $N \rightarrow \infty$ é dada pelo seguinte problema variacional:

$$
E^{V} \equiv \inf _{\mu \in \mathcal{M}(\mathbb{C})} I^{V}(\mu), \quad E<\infty
$$

onde o ínfimo é tomado sobre o conjunto $\mathcal{M}(\mathbb{C})$ de todas as medidas de probabilidade em $\mathbb{C}$. No caso de existir uma medida de probabilidade $\mu^{V}$ para cada potencial $V$ tal que

$$
E^{V}=I\left(\mu^{V}\right)
$$

denominamos $\mu^{V}$ a medida de equilíbrio associada à $V$.

Observação 17 De acordo com a Observação 16, $\mu^{V}$ é a distribuição limite de cargas correspondente ao limite termodinâmico $(N \rightarrow \infty)$ e de temperatura zero $\left(T^{-1} \rightarrow \infty\right)$ de um gás de Coulomb bidimensional à temperatura $T=N^{-1}$ (para uma discussão mais detalhada, veja [14, 9, 15]). 
Para uma classe ampla de potenciais $V$ satisfazendo certas hipóteses que enunciaremos no Capítulo 2, existe uma única medida de equilíbrio $\mu^{V}$ com suporte $\mathbb{S}^{V} \subset \mathbb{C}$ compacto (ou seja, $\mathbb{S}^{V}$ é um conjunto fechado e limitado). Além disso, caso $V$ tenha segundas derivadas parciais contínuas, $\mu^{V}$ é absolutamente contínua com respeito à medida de Lebesgue no plano complexo e pode ser escrita em termos do potencial $V$.

A conexão entre matrizes aleatórias e polinômios ortogonais se dá quando escrevemos a equação (1.21) como um determinante

$$
P_{N}\left(z_{1}, \cdots, z_{N}\right)=\frac{1}{N !} \operatorname{det}\left(K_{N}\left(z_{i}, z_{j}\right)\right)_{i, j=1}^{N}
$$

do núcleo integral

$$
K_{N}(z, w)=e^{-\frac{N}{2} V(z)} e^{-\frac{N}{2} \overline{V(w)}} \sum_{j=1}^{N} \phi_{j}^{N}(z) \overline{\phi_{j}^{N}(w)}
$$

onde $\left\{\phi_{j}^{N}\right\}_{j=1}^{N}$ é o conjunto dos polinômios ortonormais com respeito ao peso

$$
d \nu(z)=e^{-N V(z)} d^{2} z
$$

gerados a partir dos monômios pelo método de Gram-Schmidt. A demonstração deste fato se encontra na Seção 3.1.2.

Definindo a função de correlação de $n$-pontos associada à $P_{N}$ da mesma forma que é definido em sistemas contínuos em Mecânica Estatística (veja, por exemplo, [16])

$$
R_{n}^{N}\left(z_{1}, \cdots, z_{n}\right)=\frac{N !}{(N-n) !} \int P_{N}\left(z_{1}, \cdots, z_{N}\right) \prod_{i=n+1}^{N} d^{2} z_{i}
$$

com $P_{N}$ dado por (1.28), demonstramos na Seção 3.1.3 que a função de n-pontos é também um determinante do mesmo núcleo integral:

$$
R_{n}^{N}\left(z_{1}, \cdots, z_{n}\right)=\operatorname{det}\left(K_{N}\left(z_{i}, z_{j}\right)\right)_{i, j=1}^{n}
$$

Conseqüentemente, o comportamento assintótico do núcleo integral $K_{N}$ governa a estatística dos autovalores de um ensemble unitário normal quando $N \rightarrow \infty$.

\subsection{Problema de balayage}

O problema de balayage ${ }^{1}$ pode ser enunciado da seguinte forma: encontre uma medida de probabilidade $\widehat{\mu}^{V}$ (medida de balayage associada à $\mu^{V}$ ) com suporte em $\partial \mathbb{S}^{V}$ (borda

\footnotetext{
${ }^{1}$ Balayage é uma palavra francesa para varrição ou sweeping out do Inglês. O sentido empregado é varrer as cargas do interior do domínio para a fronteira.
} 
do suporte $\mathbb{S}^{V}$ de $\mu^{V}$ ) tal que o potencial logarítmico (1.26) não se altere:

$$
U^{\mu^{V}}=U^{\widehat{\mu}^{V}} \text { quase por toda parte em } \partial \mathbb{S}^{V} .
$$

A interpretação eletrostática para a medida de balayage $\widehat{\mu}^{V}$ é a seguinte: dada a medida de equilíbrio $\mu^{V}$ com suporte $\mathbb{S}^{V}, \widehat{\mu}^{V}$ é a densidade de cargas associadas a $\mu^{V}$ tal que o potencial eletrostático devido a estas se mantenha inalterado em $\partial \mathbb{S}^{V}$ quando a "varremos" de $\mathbb{S}^{V}$ para a borda $\partial \mathbb{S}^{V}$. Este conceito de medida de balayage será importante para estabelecermos uma relação entre o ensemble unitário normal e os ensembles unitário hermitiano e circular.

\subsection{Resumo dos resultados obtidos e roteiro de leitura}

Todos os teoremas, corolários e definições enunciados nessa seção são originais.

A presente Tese está dividida em três partes. A primeira explora uma propriedade curiosa das medidas de equilíbrio no que diz respeito a sua projeção em um subconjunto singular. Serão elaborados dois conceitos de projeção, sendo que o primeiro e mais importante deles é baseado na medida de balayage e o outro relacionado com a medida marginal.

A segunda parte desenvolve o cálculo do núcleo integral e do seu comportamento assintótico para alguns ensembles unitários normais.

Finalmente, a terceira parte aborda a questão da universalidade no ensemble unitário normal. Este conceito de universalidade já é bem estabelecido para o ensemble unitário hermitiano e aqui estendemos este conceito para o ensemble unitário normal.

\subsubsection{Relação entre ensembles de matrizes aleatórias}

Definição 18 Um ensemble unitário normal será chamado de ensemble singular se e somente se o suporte $\mathbb{S}^{V}$ da medida de equilíbrio for uma curva em $\mathbb{C}$.

Como exemplos de ensembles singulares temos os ensembles unitários hermitianos $\left(\right.$ com $\mathbb{S}^{V}$ compacto contido em $\mathbb{R}$ ) e o ensemble circular (com $\mathbb{S}^{V}$ a circunferência unitária).

A relação entre os ensembles não singulares e singulares será feita quando identificarmos a medida de balayage de um ensemble não singular com a medida de equilíbrio de um ensemble singular. Notemos que para que este tipo de relação seja possível devemos ter necessariamente que a borda do suporte da medida de equilíbrio do ensemble não singular coincida com o suporte do ensemble singular considerado. 
Assim se $\partial \mathbb{S}^{V}$ for um segmento de reta no eixo real, podemos associar esta medida de balayage a um ensemble unitário hermitiano (pois este ensemble possui autovalores escalados em compactos contidos na reta real) e quando a curva $\partial \mathbb{S}^{V}$ for a circunferência unitária, podemos associar esta medida de balayage ao ensemble circular de Dyson.

Teorema 19 Seja $\mu^{V}$ a medida de equilíbrio associada à $V$ (veja equação (1.27)), $\mathbb{S}^{V}$ seu suporte compacto, $\mathbb{S}_{+}^{V}=\mathbb{S}^{V} \backslash \partial \mathbb{S}^{V}$ o interior de $\mathbb{S}^{V}$ e $V$ suave o suficiente para que $\partial \mathbb{S}^{V}$ seja uma curva analítica com função de Schwarz $S(z)$. Então, a medida de balayage $\widehat{\mu}^{V}$ associada à $\mu^{V}$ é absolutamente contínua em $\partial \mathbb{S}^{V}$ com respeito à medida de comprimento de arco $d z$ em $\partial \mathbb{S}^{V}$, e é dada por

$$
d \widehat{\mu}^{V}(z)=\left.\frac{1}{2 \pi i} \partial_{z} V(z)\right|_{\bar{z}=S(z)} d z .
$$

em $\partial \mathbb{S}^{V}$.

Outras condições além de suavidade sobre $V$ serão impostas para a prova deste Teorema. A função de Schwarz de uma dada curva analítica $\gamma$ é a única função analítica $S(z)$ tal que

$$
S(z)=\bar{z} \text { para } z \in \gamma
$$

A demonstração deste Teorema se encontra na Seção 2.2 .

Este Teorema será de fundamental importância para estabelecermos relações entre as medidas de balayage de ensembles não singulares com medidas de equilíbrio de ensembles singulares.

Como simples conseqüência do Teorema 19, temos:

Corolário 20 Sejam $V_{1}$ e $V_{2}$ potenciais como no Teorema anterior. Se $\partial_{z} V_{1}=\partial_{z} V_{2}$ e os suportes $\mathbb{S}^{V_{1}}$ e $\mathbb{S}^{V_{2}}$ das medidas de equilibrio $\mu^{V_{1}}$ e $\mu^{V_{2}}$ associadas aos potenciais $V_{1}$ e $V_{2}$ têm a mesma borda analítica, então as respectivas medidas de balayage $\widehat{\mu}^{V_{1}}$ e $\widehat{\mu}^{V_{2}}$ são as mesmas.

\section{Aplicações}

Com o Teorema 19 em mãos, podemos relacionar a distribuição de autovalores de ensembles unitários normais com a distribuição de ensembles unitários hermitianos e o ensembles circular: 
Teorema 21 Seja $\widehat{\mu}^{V}$ a medida de balayage associada à medida de equilíbrio $\mu^{V}$ com e $V: \mathbb{C} \rightarrow \mathbb{R}$ um potencial dado por

$$
V(z) \equiv V_{C}(r), z=r e^{i \theta}
$$

onde $V_{C}: \mathbb{R}^{+} \cup\{0\} \rightarrow \mathbb{R}$ tal que $V_{C}^{\prime}(r)$ é absolutamente contínua com respeito à medida de Lebesgue em $\mathbb{R}^{+}, r V_{C}^{\prime}(r)$ é crescente em $\mathbb{R}^{+}, V_{C}^{\prime}(0) \geq 0$ e $V_{C}^{\prime}(1)=2$. Então o suporte $\mathbb{S}^{V}$ de $\mu^{V}$ é o disco unitário

$$
\mathbb{S}^{\mu^{V}}=\left\{z=r e^{i \theta} \in \mathbb{C}: 0 \leq r \leq 1\right\}
$$

$e$

$$
d \widehat{\mu}^{V}(z)=\frac{d \theta}{2 \pi}, z=r e^{i \theta}
$$

Em particular, isto implica que a medida de balayage associada à medida de equilíbrio do ensemble normal de Ginibre (veja 1.4) coincide com a medida de equilíbrio do ensemble circular de Dyson (veja 1.7). A demonstração deste Teorema se encontra na Seção 2.3.

A relação entre ensembles de matrizes normais e mapeamentos conformes foi descoberta por Wiegmann e Zabrodin [17], sendo estabelecida rigorosamente por Elbau e Felder [18] pela restrição a matrizes com espectro em um domínio limitado. Em [18], Elbau e Felder estudaram a medida de equilíbrio de ensembles normais sujeitos a potenciais $V$ com a forma

$$
V_{n}(z)=\frac{1}{t_{0}}\left(z \bar{z}-\sum_{k=2}^{n+1}\left(t_{k} z^{k}+\bar{t}_{k} \bar{z}^{k}\right)\right) .
$$

Para estes ensembles temos:

Teorema 22 A medida de balayage $\widehat{\mu}^{V_{n}}$ associada à medida de equilíbrio $\mu^{V_{n}}$ com suporte em $\mathbb{S}^{V_{n}} \dot{e}$

$$
d \widehat{\mu}^{V_{n}}(z)=\frac{1}{2 \pi i t_{0}} S(z) d z
$$

onde $S(z)$ é a função de Schwarz associada à $\partial \mathbb{S}^{V_{n}}$ e dz é a medida de comprimento de $\operatorname{arco}$ em $\partial \mathbb{S}^{V_{n}}$.

A demonstração deste Teorema se encontra na Seção 2.4.

Este Teorema nos diz que a medida de balayage associada ao ensemble de Elbau-Felder é proporcional a função de Schwarz da borda do suporte da medida de equilíbrio desse ensemble. Usaremos este fato para relacionarmos o ensemble de Ginibre com o ensemble de Wigner. 
Se tomarmos $n=1, t_{0}=1-\tau^{2}$ e $2 t_{2}=\tau$ para $\tau \in[0,1[$ em (1.30), temos

$$
V_{\tau}(z)=\frac{x^{2}}{1+\tau}+\frac{y^{2}}{1-\tau} \text { onde } z=x+i y \operatorname{com} x, y \in \mathbb{R}
$$

e a família de ensembles a 1 parâmetro $\tau \in$ ]0, 1[ é denominado ensemble normal de Girko. Notemos que para $\tau=0$ obtemos o ensemble normal de Ginibre conforme Observação 15. A medida de equilíbrio destes ensembles é dada por

$$
d \mu^{V_{\tau}}(z)=\frac{1}{\pi\left(1-\tau^{2}\right)} \chi_{\mathbb{S} V_{\tau}}(z) d^{2} z
$$

onde $\chi_{\mathbb{A}}$ é a função indicadora do conjunto $\mathbb{A} \mathrm{e}$

$$
\mathbb{S}^{V_{\tau}}=\left\{z \in \mathbb{C}: \frac{x^{2}}{(1+\tau)^{2}}+\frac{y^{2}}{(1-\tau)^{2}} \leq 1\right\}
$$

é o suporte de $\mu^{V_{\tau}}$. Uma aplicação do Teorema anterior para o potencial $V_{\tau}$ resulta no seguinte:

Teorema 23 Se $\mu^{V_{\tau}}$ é a medida de equilíbrio do ensemble de Girko com $V_{\tau}$ e $\mathbb{S}^{V_{\tau}}$ como acima, então

$$
\int_{\mathbb{S} V_{\tau}} f(z) d \mu^{V_{\tau}}(z)=\frac{1}{2 \pi \tau} \int_{-2 \sqrt{\tau}}^{2 \sqrt{\tau}} f(x) \sqrt{4 \tau-x^{2}} d x
$$

vale para todo $\tau \in] 0,1\left[\right.$ e toda função holomorfa $f$ em $\mathbb{S}_{+}^{V_{\tau}}=\mathbb{S}^{V_{\tau}} \backslash \partial \mathbb{S}^{V_{\tau}}$ e contínua em $\mathbb{S}^{V_{\tau}}$. Em particular, para $\tau \rightarrow 1$ temos

$$
\lim _{\tau \rightarrow 1} \mu^{V_{\tau}}=\mu_{W}
$$

(no sentido da equação (1.32)), onde

$$
\mu_{W}(x)=\frac{1}{2 \pi} \sqrt{4-x^{2}} \chi_{[-2,2]}(x)
$$

é a lei do semicírculo de Wigner. Além disso,

$$
\lim _{\tau \rightarrow 1} V_{\tau}(x+0 i)=V_{W}(x)=\frac{x^{2}}{2}, x \in \mathbb{R}
$$

$e$

$$
\lim _{\tau \rightarrow 1} V_{\tau}(z)=\infty \forall z \in \mathbb{C} \backslash \mathbb{R} .
$$

$\mu_{W}$ é a medida de equilíbrio associada ao potencial $V_{W}$ no problema variacional (1.27) em $\mathbb{R}$.

O Teorema 23 é a forma variacional de se estabelecer a correspondência entre ensembles e pode ser utilizado para se investigar o "crossover" estudado em [19]. 
Este resultado se diferencia da simples projeção ortogonal (discutida na última Seção do Capítulo 2) não só pelo fato de termos um ensemble que interpola duas situações distintas (ensemble normal de Girko), mas também porque durante todo o processo de deformação conforme do suporte $\mathbb{S}^{V_{\tau}}, \tau \in[0,1]$, a capacidade (Definição 38 a seguir) de $\mathbb{S}^{V_{\tau}}$ é preservada. Na interpretação eletrostática a transformação parametrizada por $\tau$ preserva a energia eletrostática do sistema.

O Teorema 23 será demonstrado na Seção 2.5.

Outra conexão entre ensembles de matrizes normais e hermitianas é dada pelo Teorema:

Teorema 24 (Projeção Ortogonal) Sejam $\mu_{N}$ e $\mu_{H}$ a medida de equilíbrio para os potenciais $V_{N}: \mathbb{C} \rightarrow \mathbb{R}$

$$
V_{N}(z)=\frac{2}{\alpha} r^{\alpha}, \alpha>0, z=r e^{i \theta} \in \mathbb{C}
$$

$e V_{H}: \mathbb{R} \rightarrow \mathbb{R}$

$$
V_{H}(x)=\gamma_{\alpha}|x|^{\alpha}, \alpha>0, \gamma_{\alpha}=\frac{\Gamma\left(\frac{\alpha}{2}\right) \Gamma\left(\frac{1}{2}\right)}{\Gamma\left(\frac{\alpha+1}{2}\right)}, x \in \mathbb{R}
$$

respectivamente. Então

$$
d \mu_{H}(x)=\int_{L_{x}} d \mu_{N}(z)
$$

onde

$$
L_{x}=\{z=x+i y \in \mathbb{D}: x \text { fixo }\} \text { e } \mathbb{D}=\{z \in \mathbb{C}:|z| \leq 1\}
$$

A prova deste Teorema se encontra na Seção 2.5.1.

\subsubsection{Núcleo integral de alguns ensembles normais}

Teorema 25 Seja $V: \mathbb{C} \rightarrow \mathbb{R}$ um potencial radialmente simétrico:

$$
V(z) \equiv V_{C}(r), \quad z=r e^{i \theta}
$$

satisfazendo as condições enunciadas no Teorema 40. Então $\left\{\phi_{j}^{N}\right\}_{j=1}^{N}$ com

$$
\phi_{j}^{N}(z)=\frac{z^{j-1}}{\sqrt{2 \pi \int_{0}^{\infty} r^{2 j-1} e^{-N V_{C}(r)} d r}}
$$

forma um conjunto ortonormal com respeito ao peso

$$
d \nu(z)=e^{-N V(z)} d^{2} z
$$


e, conseqüentemente, o núcleo integral (1.29) é dado por

$$
K_{N}^{C}(z, w)=e^{-\frac{N}{2} V_{C}(|z|)} e^{-\frac{N}{2} \overline{V_{C}(|w|)}} \sum_{j=1}^{N} \frac{(z \bar{w})^{j-1}}{2 \pi \int_{0}^{\infty} x^{2 j-1} e^{-N V_{C}(x)} d x} .
$$

A demonstração deste Teorema se encontra na Seção 3.2 .

Este resultado nos diz que os polinômios ortogonais de ensembles normais com potenciais radialmente simétricos são na verdade monômios, o que simplifica a sua manipulação em casos mais explícitos, como o caso abaixo.

Para a família de potenciais do tipo

$$
V_{\alpha}(z)=|z|^{\alpha}
$$

temos algo mais explícito:

Corolário 26 Os monômios $\left\{\phi_{j}^{\alpha}\right\}_{j=1}^{N}$, com

$$
\phi_{j}^{\alpha}(z)=\frac{N^{\frac{j}{\alpha}} z^{j-1}}{\sqrt{\frac{2 \pi}{\alpha} \Gamma\left(\frac{2 j}{\alpha}\right)}}
$$

formam um conjunto ortonormal com respeito ao peso

$$
d \nu^{\alpha}(z)=e^{-N V_{\alpha}(z)} d^{2} z
$$

onde $V_{\alpha}: \mathbb{C} \rightarrow \mathbb{R}$ é a família de potenciais do tipo

$$
V_{\alpha}(z)=|z|^{\alpha}, \alpha>0
$$

Então

$$
K_{N}^{\alpha}(z, w)=\frac{\alpha e^{-\frac{N}{2}|z|^{\alpha}} e^{-\frac{N}{2}|w|^{\alpha}}}{2 \pi} \sum_{j=1}^{N} \frac{N^{\frac{2 j}{\alpha}}(z \bar{w})^{j-1}}{\Gamma\left(\frac{2 j}{\alpha}\right)} .
$$

Veja na Seção 3.2.1 para a demonstração deste Teorema.

Para este caso, obtemos seu comportamento assintótico:

Teorema 27 O núcleo integral $K_{N}^{\alpha}$ associado à família de potenciais radialmente simétrico do tipo

$$
V_{\alpha}(z)=|z|^{\alpha}
$$

satisfaz para cada $\frac{1}{2}<\delta<1$ a relação assintótica

$$
\frac{1}{N^{\delta+2 \gamma}} K_{N}^{\alpha}\left(\frac{Z}{N^{\gamma}}, \frac{W}{N^{\gamma}}\right)=\frac{\alpha^{2}}{4 \pi}(Z \bar{W})^{\frac{\alpha}{2}-1} e^{N^{\delta}\left((Z \bar{W})^{\frac{\alpha}{2}}-\frac{|Z|^{\alpha}}{2}-\frac{|W|^{\alpha}}{2}\right)}(1+R)
$$


com

$$
R=O\left(N^{1-2 \delta}\right)+O\left(\frac{\log N}{N^{\frac{\delta}{2}}}\right)
$$

e $\gamma$ tal que

$$
\alpha \gamma+\delta=1
$$

Em particular, tomando $\delta \nearrow 1$ e conseqüentemente $\gamma \searrow 0$ obtemos

$$
\frac{1}{N} K_{N}^{\alpha}(Z, W)=\frac{\alpha^{2}}{4 \pi}(Z \bar{W})^{\frac{\alpha}{2}-1} e^{N\left((Z \bar{W})^{\frac{\alpha}{2}-\frac{|Z|^{\alpha}}{2}-\frac{|W|^{\alpha}}{2}}\right)}\left(1+O\left(\frac{\log N}{\sqrt{N}}\right)\right) .
$$

Veja Seção 3.2.1 para a demonstração deste Teorema.

Este Teorema pode ser colocado como principal resultado desta tese, pois ele é de fundamental importância para todo o desenvolvimento dos resultados envolvendo universalidade para o ensemble normal (próxima seção). Além disso, obtemos o termo de correção na ordem $N$. Para termos de comparação, o resultado conhecido na literatura era apenas o caso $\alpha=2$ sem o termo de correção na ordem $N$ (veja Apêndice E.1 Teorema E.6 para $\tau=0)$.

\subsubsection{Universalidade para o ensemble unitário normal}

O conceito de universalidade para o ensemble unitário hermitiano usado por Deift at. al. em [20] é o seguinte: seja

$$
P_{N}\left(\lambda_{1}, \cdots, \lambda_{N}\right)=\frac{1}{Z_{N}} e^{-N \sum_{i=1}^{N} V\left(\lambda_{i}\right)} \prod_{1 \leq i<j \leq N}\left|\lambda_{i}-\lambda_{j}\right|^{2} \prod_{1 \leq i \leq N} d \lambda_{i}
$$

a distribuição marginal conjunta do onde ensemble unitário hermitiano, onde

$$
Z_{N}=\int_{\lambda_{1}<\cdots<\lambda_{N}} e^{-\sum_{i=1}^{N} V\left(\lambda_{i}\right)} \prod_{1 \leq i<j \leq N}\left|\lambda_{i}-\lambda_{j}\right|^{2} \prod_{1 \leq i \leq N} d \lambda_{i} .
$$

Então, dizemos que o ensemble unitário hermitiano é universal porque o limite para o núcleo integral (análogo a (1.35) para este ensemble) é independente de $V$, na escala onde os autovalores têm "espaçamento" médio unitário. Isso implica que a distribuição dos espaçamentos dos autovalores nesta escala seja independente de $V$.

Seguindo estas idéias, generalizaremos este conceito para a família de ensembles unitários normais com potenciais do tipo

$$
V_{\alpha}(z)=|z|^{\alpha}
$$

O "espaçamento" médio unitário é definido da seguinte maneira: 
Teorema 28 Para a família de ensembles unitários normais com potencial $V_{\alpha}$ dado por (1.36) a função de escala

$$
g_{\alpha}(s)=\left(\frac{2 \pi s^{2}}{N \alpha}\right)^{\frac{1}{\alpha}}
$$

deixa os autovalores com espaçamento unitário uniformemente em s. Mais precisamente, se

$$
f_{N}(s, \alpha)=N^{-1} \sum_{i=1}^{N} \chi_{\mathbb{A}(s, \alpha)}\left(\lambda_{i}\right)
$$

denota a fração dos autovalores contidos no conjunto

$$
\mathbb{A}(s, \alpha)=\left\{z \in \mathbb{C}:|z| \leq g_{\alpha}(s)\right\}
$$

então

$$
\mathbb{E}\left[N f_{N}(s, \alpha)\right]=\pi s^{2}(1+o(1))
$$

com a esperança $\mathbb{E}$ tomada com respeito à distribuição

$$
P_{N}^{V_{\alpha}}\left(z_{1}, \cdots, z_{N}\right)=Z_{N}^{-1} \prod_{1 \leq i<j \leq N}\left|z_{i}-z_{j}\right|^{2} e^{-N \sum_{k=1}^{N} V_{\alpha}\left(z_{k}\right)}
$$

Este Teorema será provado na Seção 4.2.

Enunciaremos a seguir o limite para o núcleo integral (1.35) na escala onde os autovalores têm "espaçamento" médio unitário.

Teorema 29 Sejam $K_{N}^{\alpha}(z, w)$ e $g_{\alpha}(s)$ como em (1.35) e (1.37) e definimos

$$
\widetilde{K}_{N}^{\alpha}(z, w)=g_{\alpha}^{\prime}(z) K_{N}^{\alpha}\left(g_{\alpha}(z), g_{\alpha}(w)\right) \overline{g_{\alpha}^{\prime}(w)} .
$$

para cada $z, w \in \mathbb{C}$. Então o núcleo integral limite

$$
\lim _{N \rightarrow \infty} \widetilde{K}_{N}^{\alpha}(z, w)=S^{\alpha}(z, w)
$$

converge para a função

$$
S^{\alpha}(z, w)=\frac{2}{\alpha} e^{\frac{2 \pi}{\alpha}\left(z \bar{w}-\frac{|z|^{2}}{2}-\frac{|w|^{2}}{2}\right)}
$$

dependente de $\alpha \in] 0, \infty[$.

A prova deste Teorema é dada na Seção 4.3.

Calculamos também a probabilidade de não haver autovalores em uma bola aberta centrada na origem de raio $r$ :

$$
\mathbb{B}_{0, r}=\{z \in \mathbb{C}:|z|<r\}
$$


Teorema 30 A probabilidade

$$
E_{N}^{V_{\alpha}}(r)=\int P_{N}^{V_{\alpha}}\left(z_{1}, \cdots, z_{N}\right) \prod_{k=1}^{N} \chi_{\mathbb{R} \backslash \mathbb{B}_{0, r}}\left(z_{k}\right) d^{2} z_{k}
$$

de não haver autovalores na bola $\mathbb{B}_{0, r}$ na família de ensembles unitários normais com potencial $V_{\alpha}$ é dado por

$$
E_{N}^{V_{\alpha}}(r)=\prod_{k=1}^{N} \frac{\Gamma\left(\frac{2 k}{\alpha}, N r^{\alpha}\right)}{\Gamma\left(\frac{2 k}{\alpha}\right)}
$$

onde

$$
\Gamma(x, r)=\int_{r}^{\infty} t^{x-1} e^{-t} d t \text { e } \Gamma(x)=\Gamma(x, 0) .
$$

A prova deste Teorema é dada na Seção 4.4.

Também calculamos a probabilidade escalada:

Corolário 31 Sejam $E_{N}^{V_{\alpha}}(r)$ e $g_{\alpha}(s)$ como em (1.43) e (1.37) respectivamente. Então

$$
E_{N}^{V_{\alpha}}\left(g_{\alpha}(s)\right)=\prod_{k=1}^{N} \frac{\Gamma\left(\frac{2 k}{\alpha}, \frac{2 \pi s^{2}}{\alpha}\right)}{\Gamma\left(\frac{2 k}{\alpha}\right)}
$$

e, conseqüentemente,

$$
\lim _{N \rightarrow \infty} E_{N}^{V_{\alpha}}\left(g_{\alpha}(s)\right)=\prod_{k=1}^{\infty} \frac{\Gamma\left(\frac{2 k}{\alpha}, \frac{2 \pi s^{2}}{\alpha}\right)}{\Gamma\left(\frac{2 k}{\alpha}\right)}
$$

O resultado acima é consistente com o fato do limite

$$
\lim _{N \rightarrow \infty} \widetilde{K}_{N}^{\alpha}(z, w)=S^{\alpha}(z, w)
$$

depender de $\alpha$.

A seguir mostraremos que neste ensemble normal não podemos exigir espaçamento unitário dos autovalores para obtermos um núcleo integral universal.

\section{Universalidade Conforme}

A seção anterior nos diz que o conceito de universalidade empregado no ensemble unitário hermitiano não pode ser extrapolado diretamente para o ensemble unitário normal. Assim, nós propomos um novo conceito de universalidade:

Definição 32 Seja

$$
K_{N}^{V_{\alpha}}(z, w)=\widehat{K}_{N}^{V_{\alpha}}(z, w)(1+o(1))
$$


o núcleo integral do ensemble normal associado a uma família a um parâmetro de potenciais $V_{\alpha}$. Esta família de ensembles é dita ser universal se existe uma transformação conforme $\varphi_{V_{\alpha}, N}$ e um núcleo integral universal $K^{U}$ para esta familia tal que

$$
\widehat{K}_{N}^{V_{\alpha}}(z, w)=\varphi_{V_{\alpha}, N}^{\prime}(z) K^{U}\left(\varphi_{V_{\alpha}, N}(z), \varphi_{V_{\alpha}, N}(w)\right) \overline{\varphi_{V_{\alpha}, N}^{\prime}(w)} .
$$

Alternativamente, seja $S^{V_{\alpha}}$ o núcleo integral escalado (de forma que o espaçamento médio entre os autovalores seja unitário) associado à familia $V_{\alpha}$. Então existe uma transformação linear conforme $\varphi_{V_{\alpha}}$ tal que

$$
S^{V_{\alpha}}(z, w)=\varphi_{V_{\alpha}}^{\prime}(z) K^{U}\left(\varphi_{V_{\alpha}}(z), \varphi_{V_{\alpha}}(w)\right) \overline{\varphi_{V_{\alpha}}^{\prime}(w)}
$$

No caso em que

$$
V_{\alpha}(z)=|z|^{\alpha}
$$

e, portanto

$$
K_{N}^{\alpha}(z, w)=\frac{\alpha^{2}}{4 \pi} N(z \bar{w})^{\frac{\alpha}{2}-1} e^{N\left((z \bar{w})^{\frac{\alpha}{2}}-\frac{|z|^{\alpha}}{2}-\frac{|w|^{\alpha}}{2}\right)}\left(1+O\left(\frac{\log N}{\sqrt{N}}\right)\right)
$$

obtemos

$$
\begin{gathered}
K^{U}(z, w)=\frac{1}{\pi} e^{z \bar{w}-\frac{|z|^{2}}{2}-\frac{|w|^{2}}{2}}, \\
\varphi_{V_{\alpha}, N}(z)=\sqrt{N} z^{\frac{\alpha}{2}}
\end{gathered}
$$

e

$$
\varphi_{V_{\alpha}}(z)=\sqrt{\frac{\alpha}{2 \pi}} z
$$

Além disso, mostramos também que

$$
\widehat{K}_{N}^{V_{\alpha}}(z, w)=\frac{\alpha^{2}}{4 \pi} N(z \bar{w})^{\frac{\alpha}{2}-1} e^{N\left((z \bar{w})^{\frac{\alpha}{2}}-\frac{|z|^{\alpha}}{2}-\frac{|w|^{\alpha}}{2}\right)}
$$

é um núcleo reprodutor no espaço $\mathcal{H}\left(\widetilde{\mathbb{C}}, e^{-\left|\varphi_{V_{\alpha}, N}(z)\right|^{2}}\right)$ das funções do tipo

$$
f(z) e^{-\frac{\left|\varphi_{V_{\alpha}, N}(z)\right|^{2}}{2}}
$$

com $f$ analítica em $\widetilde{\mathbb{C}}$ e com norma

$$
\left\|f(z) e^{-\frac{\left|\varphi_{V_{\alpha}, N}(z)\right|^{2}}{2}}\right\|=\int_{\widetilde{\mathbb{C}}}|f(z)|^{2} e^{-\left|\varphi_{V_{\alpha}, N}(z)\right|^{2}}<\infty,
$$

onde $\widetilde{\mathbb{C}}=\varphi_{V_{\alpha}, N}^{-1}(\mathbb{C})$ e que

$$
S^{\alpha}(z, w)=\frac{2}{\alpha} e^{\frac{2 \pi}{\alpha}\left(z \bar{w}-\frac{|z|^{2}}{2}-\frac{|w|^{2}}{2}\right)}
$$


é um núcleo reprodutor no espaço $\mathcal{H}\left(\mathbb{C}, e^{-\left|\varphi_{V_{\alpha}}(z)\right|^{2}}\right)$. Um núcleo integral $K$ é chamado de reprodutor num espaço de funções $\mathcal{H}$ se e somente se

$$
\int K(z, w) f(w) d^{2} w=f(z)
$$

para toda função $f \in \mathcal{H}$. Em resumo, provamos:

Teorema 33 Seja $\varphi_{V_{\alpha}, N}: \widetilde{\mathbb{C}} \rightarrow \mathbb{C}$ o mapa conforme

$$
\varphi_{V_{\alpha}, N}(z)=\sqrt{N} z^{\frac{\alpha}{2}}
$$

$e$

$$
K^{U}(z, w)=\frac{1}{\pi} e^{z \bar{w}-\frac{|z|^{2}}{2}-\frac{|w|^{2}}{2}}
$$

o núcleo reprodutor no espaço de Segal-Bargmann $\mathcal{H}\left(\mathbb{C}, e^{-|z|^{2}}\right)$. Então a ordem dominante em $N$ do núcleo integral $\widehat{K}_{N}^{V_{\alpha}}(z, w)$ (veja (1.44)) associado à família de potenciais $V_{\alpha}(z)=|z|^{\alpha}$ pode ser escrita como

$$
\widehat{K}_{N}^{V_{\alpha}}(z, w)=\varphi_{V_{\alpha}, N}^{\prime}(z) K^{U}\left(\varphi_{V_{\alpha}, N}(z), \varphi_{V_{\alpha}, N}(w)\right) \overline{\varphi_{V_{\alpha}, N}^{\prime}(w)}
$$

e, além disso, $\widehat{K}_{N}^{V_{\alpha}}(z, w)$ é um núcleo reprodutor em $\mathcal{H}\left(\widetilde{\mathbb{C}}, e^{-\left|\varphi_{V_{\alpha}, N}(z)\right|^{2}}\right)$, onde $\widetilde{\mathbb{C}}=$ $\varphi_{V_{\alpha}, N}^{-1}(\mathbb{C})$.

\section{Corolário 34}

$$
S^{\alpha}(z, w)=\varphi_{V_{\alpha}}^{\prime}(z) K^{U}\left(\varphi_{V_{\alpha}}(z), \varphi_{V_{\alpha}}(w)\right) \overline{\varphi_{V_{\alpha}}^{\prime}(w)}
$$

onde

$$
\varphi_{V_{\alpha}}(z)=\sqrt{\frac{\alpha}{2 \pi}} z
$$

Além disso, $S^{\alpha}$ é um núcleo reprodutor em $\mathcal{H}\left(\mathbb{C}, e^{-\left|\varphi_{V_{\alpha}}(z)\right|^{2}}\right)$.

A prova deste fato está no Capítulo 4.

Logo concluímos que a família de potenciais $V_{\alpha}(z)=|z|^{\alpha}$ é universal no sentido conforme.

A relação entre $K^{U}$ e $S^{\alpha}$ se dá de maneira simples: uma transformação conforme linear (pelo Corolário 34). Um argumento plausível sobre esta pequena diferença entre $K^{U}$ e $S^{\alpha}$ (que não é observada no ensemble unitário hermitiano) pode ser explicada heuristicamente pela diferença de dimensão na qual os autovalores moram. Por isso, podemos intuir que no caso hermitiano a influência da repulsão é muito mais relevante que no caso normal $\left(V_{\alpha}\right)$. Além disso, a disposição dos autovalores em equilíbrio pode mudar com a variação do parâmetro $\alpha$, devido aos diversos arranjos cristalinos estáveis possíveis no plano. No caso hermitiano (unidimensional) não temos esta diversidade. 


\section{Universalidade de G. Oas}

Em [21], G. Oas considera a seguinte medida de probabilidade para o ensemble unitário normal:

$$
\begin{aligned}
\widehat{P}_{N}\left(z_{1}, \cdots, z_{N}\right) & =Z_{N}^{-1} \prod_{1 \leq i<j \leq N}\left|z_{i}-z_{j}\right|^{2} e^{-\sum_{i=1}^{N} V\left(z_{i}\right)} \\
& =Z_{N}^{-1} e^{-\sum_{1 \leq i \neq j \leq N} \log \left|z_{i}-z_{j}\right|^{-1}-\sum_{i=1}^{N} V\left(z_{i}\right)}
\end{aligned}
$$

que difere de (1.21) apenas pela ausência do fator $N$ à frente da somatória no expoente. A escala $N$ à frente do potencial $V$ em (1.22) faz com que a energia de interação das cargas devido ao potencial externo e a energia de interação entre as cargas devido à interação logarítmica tenham ambas a mesma ordem em $N$ (veja Observação 16) sendo mais apropriada para o estudo da medida empírica (1.24). A ausência da escala $N$ à frente da segunda somatória na equação (1.47), é mais natural na investigação da probabilidade de um conjunto aberto não conter autovalores, pois, neste caso, o espaçamento médio dos autovalores permanece $O(1)$ quando $N \rightarrow \infty$. Este espaçamento médio pode tanto depender de $\alpha$ quanto não ser uniforme nesta abordagem.

Definindo de maneira análoga a probabilidade de não haver autovalores em $\mathbb{B}_{0, r}$ neste ensemble unitário normal é

$$
\widehat{E}_{N}^{V_{\alpha}}(r)=\int \widehat{P}_{N}^{V_{\alpha}}\left(z_{1}, \cdots, z_{N}\right) \prod_{k=1}^{N} \chi_{\mathbb{R} \backslash \mathbb{B}_{0, r}}\left(z_{k}\right) d^{2} z_{k} .
$$

Oas argumenta (veja [21] equação (4.32) para $V$ um polinômio em $r=|z|$ ) que $\widehat{E}_{N}^{V}(r)$ satisfaz na vizinhança da origem a expressão

$$
\widehat{E}_{N}^{V}(r)=1-c_{V} r^{2}+O\left(r^{3}\right)
$$

com $c_{V}>0$ depende apenas de $V$ para todo $N$ e $r$ tendendo a zero. Devido a este resultado o autor conclui em [22] a universalidade dos ensembles normais com potenciais radialmente simétricos. Mostraremos a seguir que esta afirmação é verdade para a família de potenciais

$$
V_{\alpha}(z)=|z|^{\alpha}
$$

Para estes ensembles podemos, além disso, calcular $c_{V_{\alpha}}$ explicitamente.

Teorema 35 Sejam $V_{\alpha}$ e $\widehat{E}_{N}^{V_{\alpha}}$ como acima. Então

$$
\widehat{E}_{N}^{V_{\alpha}}(r)=1-c_{V_{\alpha}} r^{2}+O\left(r^{3}\right),
$$


é satisfeita para $r \rightarrow 0$, uniformemente em $N$, com

$$
c_{V_{\alpha}}=\frac{1}{\frac{2}{\alpha} \Gamma\left(\frac{2}{\alpha}\right)} .
$$

Além disso, podemos generalizar esta idéia de comportamento global para $r$ grande da seguinte forma: É sabido que para o ensemble normal de Ginibre $(\alpha=2)$ temos

$$
\widehat{E}_{N}^{V_{2}}(r) \approx e^{-\frac{r^{4}}{4}}
$$

para $N$ e $r$ grandes, com $N>r$ (veja [23]). Mais precisamente

$$
\log \widehat{E}_{N}^{V_{2}}(r)=-\frac{r^{4}}{4}-r^{2}(\log r+O(1))
$$

e, portanto, podemos generalizar este conceito de universalidade para $r$ grande como

$$
\log \widehat{E}_{N}^{V_{\alpha}}(r) \approx-c_{\alpha} r^{4}
$$

Respondemos esta questão através do Teorema:

Teorema 36 Sejam $V_{\alpha}$ e $\widehat{E}_{N}^{V_{\alpha}}$ como acima. Então para $\alpha=1$

$$
\lim _{N \rightarrow \infty} \log \widehat{E}_{N}^{V_{1}}(r)=-\left(\log 2-\frac{1}{2}\right) r^{2}-\frac{1}{2} r(\log r+O(1))
$$

quando $r \rightarrow \infty$.

A prova destes dois Teoremas encontra-se no Capítulo 4.

Isto mostra que a generalização (1.48) não é verdadeira: existe uma dependência de $\alpha$ no expoente do lado direito de (1.48) e, portanto, esta proposta de universalidade no comportamento assintótico para $r$ grande não é válida. 


\section{Capítulo 2}

\section{Medidas de equilíbrio e balayage}

\subsection{Resultados preliminares conhecidos}

Os resultados contidos nesta Seção são conhecidos e estão reunidos na referência [24].

Definição 37 Se existe uma medida de probabilidade $\mu^{V}$ associada ao potencial $V: \Sigma \subset$ $\mathbb{C} \rightarrow \mathbb{R}$ tal que

$$
E^{V}=I\left(\mu^{V}\right)=\inf _{\mu \in \mathcal{M}(\Sigma)} I^{V}(\mu), E^{V} \in \mathbb{R}
$$

(veja (1.25) para definição de $I^{V}$ ), então ela é chamada de medida de equilíbrio para $V$. Denotamos por $\mathcal{M}(\Sigma)$ o conjunto de todas as medidas positivas de Borel normalizadas (medidas de probabilidade) em $\Sigma$.

Definição 38 A capacidade logarítmica de um conjunto compacto $K \subset \mathbb{C}$ é dada por

$$
\operatorname{Cap}(K)=e^{-\inf _{\mu \in \mathcal{M}(K)} I^{V}(\mu)} .
$$

$A$ capacidade de um conjunto arbitrário de Borel $B \subset \mathbb{C}$ é dada por

$$
C a p(B)=\sup \{C a p(K): K \subset B, K \text { compacto }\}
$$

Definição 39 O termo "vale quase por toda parte (q.t.p) em um conjunto $\mathbb{A}$ " será usado se o conjunto de pontos excepcionais for de capacidade zero.

Teorema 40 (Medida de equilíbrio) Seja $V: \Sigma \rightarrow \mathbb{R} \cup\{\infty\}$ um potencial definido sobre um conjunto fechado $\Sigma \subset \mathbb{C}$ tal que:

i. É semi-contínuo por baixo, ou seja, para todo ponto $z \in \Sigma$ e todo $\varepsilon>0$, existe uma vizinhança $\mathbb{V}$ de z tal que $V(w)>V(z)-\varepsilon$, para todo $w \in \mathbb{V}$. 
ii. $\Sigma_{0}=\{z \in \Sigma: V(z) \neq \infty\}$ tem capacidade positiva.

iii. Se $\Sigma$ é não limitado, então

$$
\lim _{|z| \rightarrow \infty, z \in \Sigma}(V(z)-2 \log |z|)=\infty .
$$

Consideramos o problema variacional

$$
E^{V} \equiv \inf _{\mu \in \mathcal{M}(\Sigma)} I^{V}(\mu)
$$

onde $\mathcal{M}(\Sigma)$ é o conjunto de todas as medidas de probabilidade em $\Sigma$,

$$
I^{V}(\mu) \equiv \int\left(V(z)+U^{\mu}(z)\right) d \mu(z)
$$

é a "energia eletrostática" associada à $\mu$ e

$$
U^{\mu}(z)=\int \log |z-w|^{-1} d \mu(w)
$$

o "potencial logarítmico" associado a $\mu$. Então as seguintes propriedades valem:

a. $E^{V}$ é finito.

b. Existe um único elemento $\mu^{V} \in \mathcal{M}(\Sigma)$ tal que

$$
E^{V}=I^{V}\left(\mu^{V}\right)
$$

c. O suporte $\mathbb{S}^{V}$ de $\mu^{V}$ é compacto, $\mathbb{S}^{V} \subset \Sigma_{0}$ e possui capacidade positiva.

d. Tomando

$$
F^{V} \equiv E^{V}-\frac{1}{2} \int V(z) d \mu^{V}(z)
$$

então a desigualdade

$$
U^{\mu^{V}}(z)+\frac{V(z)}{2} \geq F^{V}
$$

vale quase em toda parte em $\Sigma$.

e. A desigualdade

$$
U^{\mu^{V}}(z)+\frac{V(z)}{2} \leq F^{V}
$$

vale para todo $z \in \mathbb{S}^{V}$.

f. Em particular, para quase todo $z \in \mathbb{S}^{V}$

$$
U^{\mu^{V}}(z)+\frac{V(z)}{2}=F^{V} .
$$


A prova deste Teorema está em [24] Capítulo I Teorema 1.3 para $V=2 Q$. Na Seção B do Apêndice provaremos uma versão deste Teorema com hipóteses mais restritivas do que acima enunciadas, porém dentro das nossas necessidades.

Observação 41 Segue da regularidade interna da medida de Borel (veja [25] Teorema 2.18) e da definição de capacidade acima que se $B$ é um conjunto de Borel com capacidade nula e $\mu$ tem energia logarítmica ${ }^{1}$ finita, então $\mu(B)=0$. Em particular, a desigualdade do item d. e a igualdade do item e. do Teorema acima valem $\mu^{V}$-quase em toda parte, ou seja:

$$
\int_{\Sigma}\left(U^{\mu^{V}}(z)+\frac{V(z)}{2}\right) d \mu^{V}(z) \geq F^{V}
$$

$e$

$$
\int_{\mathbb{S}^{V}}\left(U^{\mu^{V}}(z)+\frac{V(z)}{2}\right) d \mu^{V}(z)=F^{V}
$$

Teorema 42 (Equivalência entre medidas de Borel) Sejam $\mu$ e $\nu$ duas medidas de Borel positivas e finitas com suporte compacto $\mathbb{D} \subset \mathbb{C}$ e suponhamos que os potenciais logarítmicos $U^{\mu}$ e $U^{\nu}$ satisfazem

$$
U^{\mu}(z)=U^{\nu}(z)+u(z)
$$

em quase toda parte com respeito à medida de Lebesgue em $\mathbb{C}$, onde u é uma função harmônica em $\mathbb{D}$. Então, em $\mathbb{D}$, as medidas $\mu$ e $\nu$ coincidem, ou seja,

$$
\left.\mu\right|_{\mathbb{D}}=\left.\nu\right|_{\mathbb{D}}
$$

Veja a prova deste resultado no Apêndice C.

Teorema 43 (relação entre medida de Borel e potencial logarítmico) Se o potencial logarítmico $U^{\mu}$ (2.3) de uma medida de Borel $\mu$ tem segundas derivadas parciais contínuas em um aberto limitado $\mathbb{D}$, então $\mu$ é absolutamente contínua em $\mathbb{D}$ com respeito à medida de Lebesgue $d^{2}$ z e em $\mathbb{D}$ temos a fórmula

$$
\left.d \mu\right|_{\mathbb{D}}=-\left.\frac{1}{2 \pi} \Delta U^{\mu} d^{2} z\right|_{\mathbb{D}}
$$

onde

$$
\Delta=\frac{\partial^{2}}{\partial x^{2}}+\frac{\partial^{2}}{\partial y^{2}}=4 \frac{\partial^{2}}{\partial z \partial \bar{z}}
$$

é o operador laplaciano. Aqui usamos

$$
\frac{\partial}{\partial z}=\frac{1}{2}\left(\frac{\partial}{\partial x}-i \frac{\partial}{\partial y}\right) \quad e \frac{\partial}{\partial \bar{z}}=\frac{1}{2}\left(\frac{\partial}{\partial x}+i \frac{\partial}{\partial y}\right) .
$$

\footnotetext{
${ }^{1} \mathrm{~A}$ energia logarítmica associada a $\mu$ é dada por $\int_{\Sigma} U^{\mu}(z) d \mu(z)$
} 
Veja a prova deste fato no Apêndice C.

Corolário 44 (relação entre medida de equilíbrio e o potencial) Se pegarmos $V$, $\mathbb{S}^{V}$ e $\mu^{V}$ como no Teorema 40 com a hipótese adicional $V \in C^{2}\left(\mathbb{S}_{+}^{V}\right)$, onde $\mathbb{S}_{+}^{V}=\mathbb{S}^{V} \backslash \partial \mathbb{S}^{V}$ (o interior de $\mathbb{S}^{V}$ ) é um aberto de $\mathbb{C}$ (notamos que $\mathbb{S}_{+}^{V}$ é limitado), então em $\mathbb{S}_{+}^{V}$ temos

$$
d \mu^{V}(z)=\frac{1}{4 \pi} \Delta V d^{2} z
$$

Prova. Segue do Teorema anterior e da equação (2.4).

Teorema 45 Sejam $V, \Sigma, \Sigma_{0}, F^{V}, \mu^{V}$ e $\mathbb{S}^{\mu^{V}}$ como no Teorema 40. Então, para toda medida de probabilidade $\nu$ com suporte $\mathbb{S}^{\nu}$ contido em $\Sigma$

$$
\inf _{z \in \Sigma_{0}}\left(U^{\nu}(z)+\frac{V(z)}{2}\right) \leq F^{V}
$$

$e$

$$
\sup _{z \in \mathbb{S}^{\nu}}\left(U^{\nu}(z)+\frac{V(z)}{2}\right) \geq F^{V}
$$

Se a igualdade valer em (2.7) e (2.8), então $\nu=\mu^{V}$.

Veja a prova deste fato no Apêndice C.

Teorema 46 (Equivalência entre medidas de equilíbrio) $\operatorname{Sejam~} V, \Sigma, \Sigma_{0}, F^{V}, \mu^{V}$ $e \mathbb{S}^{\mu^{V}}$ como no Teorema 40. Seja também $\nu$ uma medida de probabilidade com suporte $\mathbb{S}^{\nu} \subset \Sigma$, energia logarítmica finita e tal que

$$
U^{\nu}(z)+\frac{V(z)}{2}
$$

coincida com uma constante $F$ em quase toda parte em $\mathbb{S}^{\nu}$ e seja maior ou igual a $F$ em s. Então $\nu=\mu^{V}$ e $F=F^{V}$. A mesma conclusão se aplica se $\nu$ for uma medida de probabilidade com suporte $\mathbb{S}^{\nu} \subset \mathbb{S}^{\mu^{V}}$, energia logarítmica finita e (2.9) coincidir com $F$ em quase toda parte de $\mathbb{S}^{\mu^{V}}$.

Veja a prova deste Teorema no Apêndice C.

Observação 47 Os Teoremas desta Subseção oferecem várias maneiras, por vezes complementares, de verificar se uma dada medida de probabilidade é a medida de equilíbrio associada a um potencial $V$. No Apêndice $D$ calculamos explicitamente as medidas de equilibrio relevantes para esta Tese. 
Definição 48 (Medida de Balayage) Seja $\mathbb{G} \subset \overline{\mathbb{C}}$ um aberto com borda compacta $\partial \mathbb{G}$. Seja $\nu$ uma medida de probabilidade em $\mathbb{G}($ de outra forma, $\nu(\overline{\mathbb{C}} \backslash \mathbb{G})=0$ ) com potencial logarítmico $U^{\nu}$ finito e contínuo (veja 1.26) em $\mathbb{G}$. O problema de balayage consiste em encontrar outra medida de probabilidade $\widehat{\nu}$ com suporte em $\partial \mathbb{G}$ tal que

$$
U^{\nu}=U^{\widehat{\nu}} \text { em } \partial \mathbb{G}
$$

Doravante chamaremos de $\widehat{\nu}$ a medida de balayage associada à $\nu$.

Observação 49 Se $\mathbb{G}$ é um aberto limitado $\mathbb{G} \subset \mathbb{C}$, $\widehat{\nu}$ é única e possui as seguintes propriedades adicionais ([24] Capitulo II, Teorema 4.1):

1. $U^{\widehat{\nu}}\left(z_{0}\right)=U^{\nu}\left(z_{0}\right)$ se $z_{0} \notin \overline{\mathbb{G}}$ ou se $z_{0} \in \partial \mathbb{G}$ e $z_{0}$ é um ponto regular da borda de $\mathbb{G}$.

2. $U^{\widehat{\nu}}(z) \leq U^{\nu}(z)$ para todo $z \in \mathbb{C}$

3.

$$
\int_{\mathbb{G}} h d \nu=\int_{\partial \mathbb{G}} h d \widehat{\nu}
$$

vale para toda função h contínua em $\overline{\mathbb{G}}$ e harmônica em $\mathbb{G}$.

Observação 50 Se escolhermos $\mathbb{G}=\mathbb{S}_{+}^{V}=: \mathbb{S}^{V} \backslash \partial \mathbb{S}^{V}$ o interior do suporte compacto da medida de equilíbrio associado a $V$ e, portanto, um conjunto aberto e limitado, então a medida de balayage associada a medida de equilíbrio $\mu^{V}$ será denotada por $\widehat{\mu}^{V}$.

\subsection{Fórmula explícita para medida de balayage}

Uma curva

$$
\gamma=z(t), t \in] a, b[, \gamma \subset \mathbb{C}
$$

é chamada analítica se em cada ponto $\left.t_{0} \in\right] a, b[$ a função $z$ tem uma expansão em série

$$
z(t)=\sum_{n=0}^{\infty} b_{n}\left(t-t_{0}\right)^{n}, \quad\left|t-t_{0}\right|<\delta .
$$

A função de Schwarz para uma curva analítica $\gamma$ é a única função analítica $S(z)$ tal que

$$
S(z)=\bar{z} \text { para } z \in \gamma
$$

Enunciaremos novamente o Teorema 19 com as hipóteses em mais detalhes. 
Teorema 51 Sejam $V, \mathbb{S}^{V}$ e $\mu^{V}$ como no Teorema 40 com as hipóteses adicionais do Corolário 44, com $\mathbb{S}_{+}^{V}=\mathbb{S}^{V} \backslash \partial \mathbb{S}^{V}$ um aberto limitado de $\mathbb{C}$ e $V$ suave o suficiente para que $\partial \mathbb{S}^{V}$ seja uma curva analítica com função de Schwarz $S(z)$. Então a medida de balayage $\widehat{\mu}^{V}$ é absolutamente contínua em $\partial \mathbb{S}^{V}$ com respeito à medida comprimento de arco $\mathrm{dz}$ em $\partial \mathbb{S}^{V}$ e em $\partial \mathbb{S}^{V}$ temos a fórmula

$$
d \widehat{\mu}^{V}(z)=\left.\frac{1}{2 \pi i} \partial_{z} V(z)\right|_{\bar{z}=S(z)} d z
$$

Prova. O Teorema de Green pode ser expresso como (veja [26] pág 488)

$$
\int_{\mathbb{D}} \partial_{\bar{z}} F d^{2} z=\frac{1}{2 i} \int_{\partial \mathbb{D}} F d z
$$

onde $\mathbb{D}$ é um domínio aberto e limitado com borda $\partial \mathbb{D}$ orientada positivamente e $C^{1}$ e $F \in C^{1}(\mathbb{D})$ e contínua em $\overline{\mathbb{D}}$. Então, por (2.6) temos, $\forall f$ holomorfa em $\mathbb{S}_{+}^{V}=\mathbb{S}^{V} \backslash \partial \mathbb{S}^{V}$ e contínua em $\mathbb{S}^{V}$,

$$
\begin{aligned}
\int_{\mathbb{S}_{+}^{V}} f(z) d \mu^{V}(z) & =\frac{1}{4 \pi} \int_{\mathbb{S}_{+}^{V}} f(z) \Delta V d^{2} z \\
& =\frac{1}{4 \pi} \int_{\mathbb{S}_{+}^{V}} f(z) 4 \partial_{z} \partial_{\bar{z}} V(z) d^{2} z \\
& =\frac{1}{\pi} \int_{\mathbb{S}_{+}^{V}} \partial_{\bar{z}}\left(f(z) \partial_{z} V(z)\right) d^{2} z \\
& =\left.\frac{1}{2 i \pi} \int_{\partial \mathbb{S}^{V}} f(z) \partial_{z} V(z)\right|_{\bar{z}=S(z)} d z \\
& =\int_{\partial \mathbb{S}^{V}} f(z) d \widehat{\mu}^{V}(z)
\end{aligned}
$$

onde no penúltimo passo usamos (2.11), a hipótese de $\partial \mathbb{S}^{V}$ ser uma curva analítica com função de Schwarz $S(z)$, o fato de $\bar{z}=S(z)$ em $\partial \mathbb{S}^{V}$ e no último passo usamos a definição de medida de balayage (Definição 48 e a Observação 49 item 2), sobre a unicidade da medida de balayage para domínios abertos limitados. 


\subsection{Ensemble de Dyson e unitário normal circular- mente simétrico}

Denotaremos por ensemble de matrizes aleatórias normais com simetria circular qualquer ensemble de matrizes aleatórias normais sujeitas a um potencial do tipo

$$
V(z) \equiv V_{C}(r), z=r e^{i \theta}
$$

satisfazendo as hipóteses do seguinte Teorema:

Teorema 52 Seja $V: \Sigma=\mathbb{C} \rightarrow \mathbb{R}$ um potencial dado por

$$
V(z) \equiv V_{C}(r), z=r e^{i \theta}
$$

com $V_{C}: \mathbb{R}^{+} \cup\{0\} \rightarrow \mathbb{R}$ tal que $V_{C}^{\prime}(r)$ absolutamente contínua com respeito à medida de Lebesgue em $\mathbb{R}^{+}$e $r V_{C}^{\prime}(r)$ crescente em $\mathbb{R}^{+}$. Então, a medida de equilíbrio $\mu$ associada ̀̀ $\checkmark$ é tal que

$$
d \mu^{V}(z)=\frac{1}{4 \pi}\left(r V_{C}^{\prime}(r)\right)^{\prime} \chi_{\left[r_{0}, R_{0}\right]}(r) d r d \theta
$$

onde

$$
\mathbb{S}^{\mu^{V}}=\left\{z=r e^{i \theta} \in \mathbb{C}: r_{0} \leq r \leq R_{0}\right\}
$$

com $r_{0} \geq 0$ o menor número tal que

$$
V_{C}^{\prime}(r)>0
$$

para $r>r_{0}$ e $R_{0}$ é a menor solução da equação

$$
r V_{C}^{\prime}(r)=2
$$

O Teorema 52 será provado no Apêndice D.1.

Corolário 53 Seja $V: \Sigma=\mathbb{C} \rightarrow \mathbb{R}$ dado por

$$
V(z)=\frac{2}{\alpha}|z|^{\alpha}, \alpha>0 .
$$

Então, a medida de equilíbrio $\mu_{N}$ associada à $V_{N}$ é tal que sua densidade é dada pela distribuição

$$
d \mu(z)=\frac{\alpha}{2 \pi}|z|^{\alpha-1} \chi_{[0,1]}(r) d r d \theta
$$

Prova. Segue diretamente do Teorema anterior, com $r_{0}=0, R_{0}=1$ e $F^{V}=\frac{1}{\alpha}$. 
Observação 54 Se tomarmos $V_{C}$ como no Teorema anterior e com as propriedades adicionais $V_{C}^{\prime}(0)=0$ e $V_{C}^{\prime}(1)=2$, então o suporte da medida de equilíbrio associada é o círculo unitário:

$$
\mathbb{S}^{\mu^{V}}=\left\{z=r e^{i \theta} \in \mathbb{C}: 0 \leq r \leq 1\right\}
$$

Enunciaremos a seguir alguns resultados segundo os quais uma conexão entre ensembles de Dyson e ensembles normais com simetria circular pode ser estabelecida. O resultado obtido por Dyson (veja Seção 1.2.2) pode ser interpretado da seguinte forma:

Teorema 55 Considere o problema variacional formulado no Teorema $40 \mathrm{com}$

$$
\Sigma=\Sigma_{D}=\{z \in \mathbb{C}:|z| \leq 1\} \text { e } V_{D} \equiv 0
$$

Então, a medida de equilíbrio $\mu^{V_{D}}$ neste caso tem suporte em $\mathbb{S}^{V_{D}}=\partial \Sigma_{D} e$

$$
d \mu^{V_{D}}=\frac{d \theta}{2 \pi} .
$$

Veja a prova deste resultado no Apêndice D.2.

Lema 56 A função de Schwarz associada à circunferência unitária $\partial \Sigma_{D}\left(\Sigma_{D}\right.$ dado por 2.14) é

$$
S(z)=\frac{1}{z}
$$

Prova. Para $z=e^{i \theta} \in \partial \Sigma_{D}$

$$
\frac{1}{z}=\frac{1}{e^{i \theta}}=e^{-i \theta}=\bar{z}=S(z) .
$$

Teorema 57 Seja $\widehat{\mu}^{V}$ a medida de balayage associada à medida de equilíbrio $\mu^{V}$ com $V: \mathbb{C} \rightarrow \mathbb{R}$ um potencial dado por

$$
V(z) \equiv V_{C}(r), z=r e^{i \theta}
$$

onde $V_{C}: \mathbb{R}^{+} \cup\{0\} \rightarrow \mathbb{R}$ tal que $V_{C}^{\prime}(r)$ absolutamente contínua com respeito à medida de Lebesgue em $\mathbb{R}^{+}, r V_{C}^{\prime}(r)$ crescente em $\mathbb{R}^{+}, V_{C}^{\prime}(0) \geq 0$ e $V_{C}^{\prime}(1)=2$. Então o suporte $\mathbb{S}^{V}$ de $\mu^{V}$ é o disco unitário

$$
\mathbb{S}^{\mu^{V}}=\left\{z=r e^{i \theta} \in \mathbb{C}: 0 \leq r \leq 1\right\}
$$

$e$

$$
\widehat{\mu}^{V}=\mu^{V_{D}}
$$

com $V_{D}$ e $\mu^{V_{D}}$ dados por (2.14) e (2.15). 
Prova do Teorema 21. Pelo Teorema 52 e pela Observação 54, de fato o suporte $\mathbb{S}^{V}$ de $\mu^{V}$ é o disco unitário

$$
\mathbb{S}^{\mu^{V}}=\left\{z=r e^{i \theta} \in \mathbb{C}: 0 \leq r \leq 1\right\} .
$$

Assim, calculando a medida de balayage associada a $\mu^{V}$ por (2.13), (2.10) e (2.16) obtemos

$$
\begin{aligned}
d \widehat{\mu}^{V}(z) & =\left.\frac{1}{2 \pi i} \partial_{z} V_{C}(r)\right|_{\bar{z}=S(z)} d z \\
& =\left.\frac{1}{2 \pi i} V_{C}^{\prime}\left((z \bar{z})^{\frac{1}{2}}\right) \partial_{z}\left((z \bar{z})^{\frac{1}{2}}\right)\right|_{\bar{z}=z^{-1}} d z \\
& =\left.\frac{1}{4 \pi i} V_{C}^{\prime}\left((z \bar{z})^{\frac{1}{2}}\right) z^{-\frac{1}{2}} \bar{z}^{\frac{1}{2}}\right|_{\bar{z}=z^{-1}} d z=\frac{V_{C}^{\prime}\left(\left(z z^{-1}\right)^{\frac{1}{2}}\right)}{4 \pi i} z^{-1} d z \\
& =\frac{V_{C}^{\prime}(1)}{4 \pi i}\left(e^{i \theta}\right)^{-1} i e^{i \theta} d \theta=\frac{d \theta}{2 \pi}
\end{aligned}
$$

como queríamos. Usamos que $V_{C}^{\prime}(1)=2$ no último passo.

Exemplo 58 Se tomarmos

$$
V(z) \equiv V_{P}(r)=\sum_{k=0}^{m} a_{k} r^{n_{k}}, r=|z|
$$

para $\left\{a_{k}\right\}_{k=0}^{m}$ e $\left\{n_{k}\right\}_{k=0}^{m}$ seqüências de números reais positivos tais que

$$
V_{P}^{\prime}(1)=\sum_{k=0}^{m} a_{k} n_{k}=2,
$$

o Teorema anterior ainda se aplica. Em particular, se escolhermos $m=0, a_{0}=1$ e $n_{0}=2$ temos

$$
V(z)=|z|^{2}
$$

e a medida de equilíbrio associada a este potencial

$$
d \mu^{V}(z)=\frac{1}{\pi} \chi_{\mathbb{S} V}(z) d^{2} z
$$

com

$$
\mathbb{S}^{V}=\{z \in \mathbb{C}:|z| \leq 1\}
$$

coincide com a função correlação de 1-ponto (densidade dos autovalores) de um ensemble normal de ginibre. Esta conexão não é por acaso. Para uma prova de que a função de 1-ponto converge para a medida de equilíbrio em ensembles hermitianos veja [7], prova que foi estendida em [27] para o ensemble normal. 


\subsection{Potenciais de Elbau-Felder}

P. Elbau e G. Felder em [18] estudaram ensemble de matrizes normais com potenciais da forma

$$
V_{n}(z)=\frac{1}{t_{0}}\left(z \bar{z}-\sum_{k=2}^{n+1}\left(t_{k} z^{k}+\overline{t_{k}} \overline{z^{k}}\right)\right)
$$

com mínimo absoluto em $z=0$ não degenerado em uma região compacta $\Sigma$ contendo a origem. O resultado principal de seu trabalho é o seguinte:

Teorema 59 (Elbau-Felder) Para toda seqüência $\left\{t_{k}\right\}_{k=0}^{\infty} \subset \mathbb{C}$ com $t_{1}=0,\left|t_{2}\right|<\frac{1}{2}$ e $t_{k}=0$ para $k>n+1$ e todo domínio compacto $\Sigma \subset \mathbb{C}$ contendo a origem como ponto interior e tal que $V_{n}$ como em (2.17) é positivo em $\Sigma \backslash\{0\}$, existe $\delta>0$ tal que para todo $0<t_{0}<\delta$ a medida de equilíbrio $\mu^{V_{n}}$ em $\Sigma$ é dada por

$$
d \mu^{V_{n}}(z)=\frac{1}{\pi t_{0}} \chi_{\Sigma_{+}}(z) d^{2} z
$$

onde $\Sigma_{+} \subset \Sigma$ denota o interior de uma única curva polinomial $\gamma$ definida pelos momentos harmônicos $\left\{t_{k}\right\}_{k=0}^{\infty}$.

Veja a prova deste Teorema em [18].

Observação 60 Os momentos harmônicos $\left\{t_{k}\right\}_{k=0}^{\infty}$ de uma curva polinomial $\gamma$ (ou de maneira mais geral uma curva analítica), que circunscreve a origem são definidos por

$$
t_{k}=\frac{1}{2 \pi i k} \int_{\gamma} \bar{z} z^{-k} d z, k>0
$$

$e$

$$
t_{0}=\frac{1}{\pi} \int_{\Sigma} d^{2} z
$$

Uma curva polinomial de grau $n$ é uma curva fechada no plano complexo com parametrização $h: \gamma$ da forma

$$
h(w)=\sum_{k=-1}^{n} a_{k} w^{-k}
$$

onde $S^{1}=\{w \in \mathbb{C}:|w|=1\}$ é a circunferência unitária, $a_{-1}>0$ e $a_{n} \neq 0$.

Observação 61 Notamos que $V_{n}$ não satisfaz (exceto para $n=1$, que é o potencial de Ginibre-Girko) a hipótese iii. do Teorema 40 (equação (2.2)), mas possui um mínimo local em $z=0$. Este fato justifica a necessidade de Elbau-Felder introduzir $\delta>0$ tal que para $0<t_{0}<\delta$ exista uma curva polinomial que delimita uma região com área suficientemente pequena na qual uma fração significativa dos autovalores fiquem confinados e permaneçam próximo do mínimo do potencial (não escapem para o infinito). 
Observação 62 Notamos que $V_{n}$ e $\mu^{V_{n}}$ satisfazem a relação (2.6) em $\Sigma$.

Prova do Teorema 22. Por (2.17) temos

$$
\partial_{z} V(z)=\frac{1}{t_{0}}\left(\bar{z}-\sum_{k=2}^{n+1} k t_{k} z^{k-1}\right) .
$$

Desta equação e da equação (2.10), podemos calcular a medida de balayage associada à $\mu^{V_{n}}$ :

$$
d \widehat{\mu}^{V_{n}}(z)=\frac{1}{2 \pi i t_{0}}\left(S(z)-\sum_{k=2}^{n+1} k t_{k} z^{k-1}\right) d z .
$$

Logo $\forall f$ holomorfa em $\Sigma \backslash \gamma$ e contínua em $\Sigma$ podemos ver que o termo da soma não contribuirá para integrais de funções com $f$ com respeito a $\widehat{\mu}^{V_{n}}$ (por continuidade e pelo Teorema de Cauchy). Logo

$$
d \widehat{\mu}^{V_{n}}(z)=\frac{1}{2 \pi i t_{0}} S(z) d z
$$

como queríamos.

\subsection{Interpolação entre os ensembles de Ginibre-Girko e o de Wigner}

O propósito desta Seção é construir um ensemble normal que interpola o ensemble unitário normal de Ginibre e o ensemble unitário hermitiano de Wigner. Isto é feito através de uma família a 1 parâmetro de potenciais $V_{\tau}(\tau \in[0,1])$ cuja família de medidas de equilíbrio $\mu^{V_{\tau}}$ associada seja tal que a "energia eletrostática" permaneça constante:

$$
E^{V_{\tau}}=I\left(\mu^{V_{\tau}}\right)=\iint \log |z-w|^{-1} d \mu^{V_{\tau}}(z) \mu^{V_{\tau}}(w)+\int V(z) d \mu^{V_{\tau}}(z)=\frac{3}{4} \forall \tau \in[0,1]
$$

e, portanto, a capacidade do suporte da medida de equilíbrio $\mu^{V_{\tau}}$ permanece constante como função de $\tau$ (veja definição 38).

O potencial do ensemble normal de Ginibre-Girko é obtido tomando $n=1$ em (2.17)

$$
V(z)=\frac{1}{t_{0}}\left(z \bar{z}-t_{2} z^{2}-\bar{t}_{2} \bar{z}^{2}\right)
$$

e por (2.18), é possível mostrar que a medida de equilíbrio associada a (2.20)

$$
d \mu^{V}(z)=\frac{1}{\pi t_{0}} \chi_{\mathbb{S}^{V}}(z) d^{2} z
$$


é uniforme em $\mathbb{S}^{V}$ (veja [18])

$$
\begin{gathered}
\mathbb{S}^{V}=\left\{z \in \mathbb{C}: \frac{\left(\operatorname{Re}\left(\sqrt{t_{2}} z\right)\right)^{2}}{a^{2}}+\frac{\left(\operatorname{Im}\left(\sqrt{t_{2}} z\right)\right)^{2}}{b^{2}} \leq\left|t_{2}\right|\right\},\left|t_{2}\right|<\frac{1}{2} \\
a=\sqrt{\frac{1+2 t_{2}}{1-2 t_{2}} t_{0}} \quad \text { e } \quad b=\sqrt{\frac{1-2 t_{2}}{1+2 t_{2}} t_{0} .}
\end{gathered}
$$

É conveniente tomarmos

$$
2 t_{2}=\tau \quad \text { e } t_{0}=1-\tau^{2}, \quad \tau \in[0,1[
$$

na equação (2.20):

$$
\begin{aligned}
V_{\tau}(z) & \equiv \frac{1}{1-\tau^{2}}\left(|z|^{2}-\tau\left(\frac{z^{2}+\bar{z}^{2}}{2}\right)\right) \\
& =\frac{x^{2}}{1+\tau}+\frac{y^{2}}{1-\tau}
\end{aligned}
$$

onde $z=x+y i, x, y \in \mathbb{R}$. A medida de equilíbrio associada a este potencial é

$$
d \mu^{V_{\tau}}(z)=\frac{1}{\pi\left(1-\tau^{2}\right)} \chi_{\mathbb{S}_{+}^{V_{\tau}}}(z) d^{2} z
$$

onde

$$
\mathbb{S}^{V_{\tau}}=\left\{z \in \mathbb{C}: \frac{x^{2}}{(1+\tau)^{2}}+\frac{y^{2}}{(1-\tau)^{2}} \leq 1\right\}
$$

$\partial \mathbb{S}^{V_{\tau}}$ é uma família a um parâmetro de elipses que interpola o círculo de raio $1(\tau=0)$ e o segmento de reta no eixo real $-2<x<2(\tau \nearrow 1)$. A figura abaixo mostra $\partial \mathbb{S}^{V_{\tau}}$ para $\tau=0,0.5,0.9$ e 0.995 .

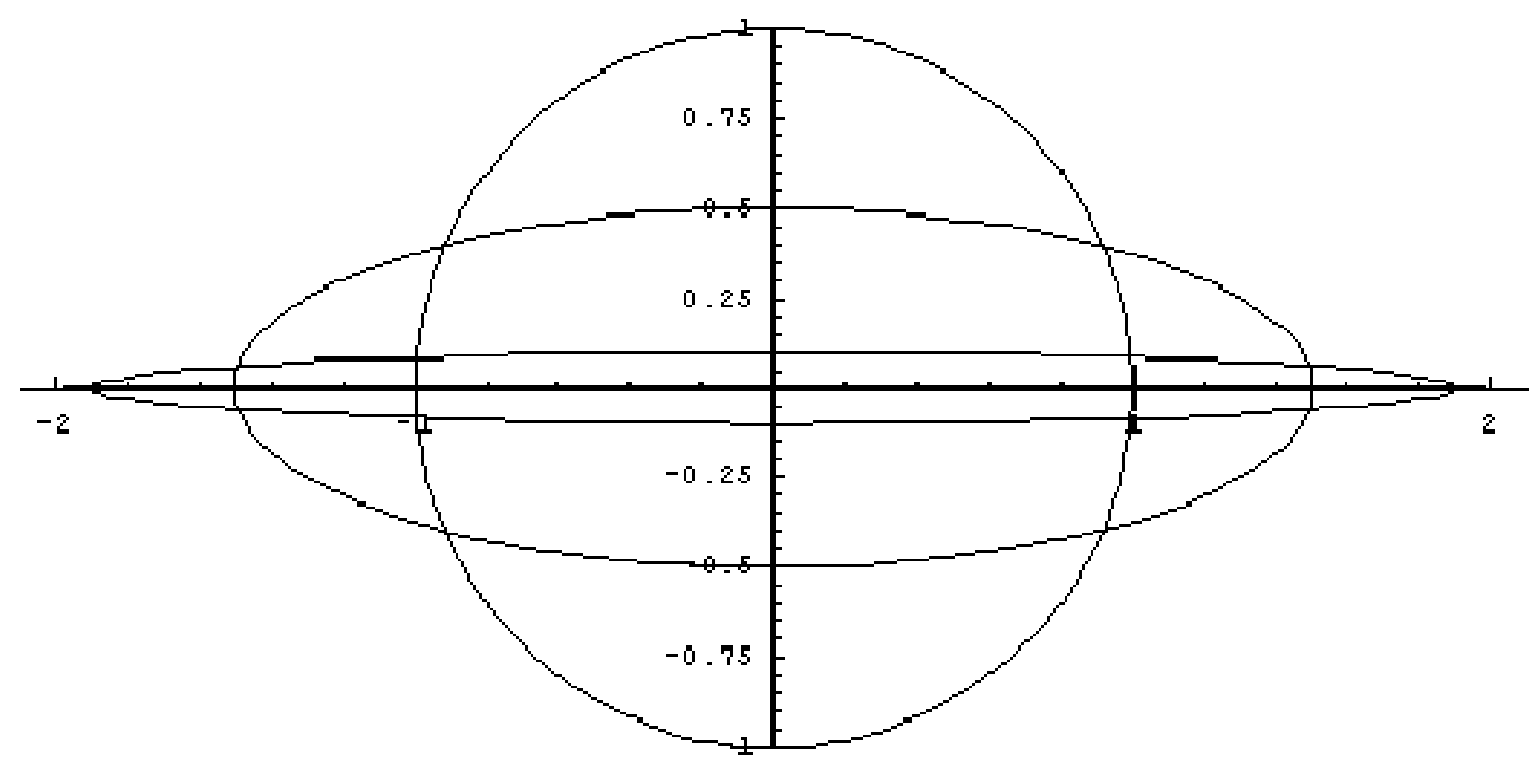


O potencial (2.23) é considerado em [19] onde é provado que para todo $\tau \in[0,1[$, a medida de equilíbrio é uniforme com suporte na elipse (2.25). Mostraremos que quando $\tau \nearrow 1$, o ensemble normal de Ginibre-Girko é projetado (no sentido discutido abaixo) no ensemble de Wigner.

Como já mencionado, o ensemble de Wigner pode ser tratado no formalismo da medida de equilíbrio com $\mathbb{C}$ substituído por $\mathbb{R}$ em (1.27), $\Sigma=\mathbb{R}$ no Teorema 40 e o potencial $V_{W}: \mathbb{R} \rightarrow \mathbb{R}$ dado por

$$
V_{W}(x)=\frac{x^{2}}{2}
$$

Assim, a medida de equilíbrio $\mu_{W}$ associada a este potencial satisfaz a lei do semicírculo

$$
d \mu_{W}(x)=\frac{1}{2 \pi} \sqrt{4-x^{2}} \chi_{[-2,2]}(x) d x .
$$

Veja Apêndice D.3 (Corolário D.6) para uma prova deste fato.

A fim de aplicarmos a fórmula deduzida no Teorema 51 para a medida de balayage associada à medida de equilíbrio $\mu^{V_{\tau}}$ do ensemble de Ginibre-Girko, devemos calcular a função de Schwarz associada à borda $\partial \mathbb{S}^{V_{\tau}}$ da elipse (2.25). Parte das idéias contidas nas demonstrações dos Teoremas a seguir estão na referência [28].

Lema 63 A função de Schwarz associada à borda da região $\partial \mathbb{S}^{V_{\tau}}$ (2.25) é

$$
\left.S(z)=\frac{1}{2}\left(\frac{1+\tau^{2}}{\tau}\right) z-\frac{i}{2}\left(\frac{1-\tau^{2}}{\tau}\right) \sqrt{4 \tau-z^{2}}, \tau \in\right] 0,1[
$$

Prova. Por (2.25), temos para $\partial \mathbb{S}^{V_{\tau}}$ (usando $z=x+i y$ )

$$
\frac{1}{(1+\tau)^{2}}\left(\frac{z+\bar{z}}{2}\right)^{2}+\frac{1}{(1-\tau)^{2}}\left(\frac{z-\bar{z}}{2 i}\right)^{2}=1
$$

e com um pouco de álgebra encontramos a seguinte equação quadrática para $\bar{z}$ :

$$
\tau \bar{z}^{2}-\left(1+\tau^{2}\right) z \bar{z}+\tau z^{2}-\left(1-\tau^{2}\right)^{2}=0
$$

Resolvendo esta equação para $\bar{z}$, temos dois candidatos à função de Schwarz:

$$
\begin{aligned}
\bar{z}_{ \pm} & =\frac{\left(1+\tau^{2}\right) z \pm \sqrt{\left(\left(1+\tau^{2}\right) z\right)^{2}-4 \tau\left(\tau z^{2}+\left(1-\tau^{2}\right)^{2}\right)}}{2 \tau} \\
& =\frac{\left(1+\tau^{2}\right) z \pm \sqrt{\left(1-\tau^{2}\right)^{2} z^{2}-4 \tau\left(1-\tau^{2}\right)^{2}}}{2 \tau} \\
& =\frac{\left(1+\tau^{2}\right) z \pm\left(1-\tau^{2}\right) i \sqrt{4 \tau-z^{2}}}{2 \tau} .
\end{aligned}
$$


Como o ponto

$$
z=i(1-\tau)
$$

pertence à borda da elipse (2.25) temos, pela definição de função de Schwarz

$$
S(i(1-\tau))=\overline{i(1-\tau)}=-i(1-\tau)
$$

Mas por outro lado, substituindo (2.30) em (2.29)

$$
\begin{aligned}
\bar{z}_{ \pm} & =\frac{\left(1+\tau^{2}\right) i(1-\tau) \pm\left(1-\tau^{2}\right) i \sqrt{4 \tau-(i(1-\tau))^{2}}}{2 \tau} \\
& =\frac{\left(1+\tau^{2}\right) i(1-\tau) \pm\left(1-\tau^{2}\right) i \sqrt{4 \tau-(i(1-\tau))^{2}}}{2 \tau} \\
& =\frac{\left(1+\tau^{2}\right) i(1-\tau) \pm\left(1-\tau^{2}\right) i(1+\tau)}{2 \tau} \\
& =\frac{i(1-\tau)}{2 \tau}\left(1+\tau^{2} \pm(1+\tau)^{2}\right)
\end{aligned}
$$

e, portanto, concluímos que

$$
S(z)=\bar{z}_{-}
$$

como queríamos.

Teorema 64 Seja $\widehat{\mu}^{V_{\tau}}$ a medida de balayage associada à (2.24). Então para $z \in \partial \mathbb{S}^{V_{\tau}}$

$$
d \widehat{\mu}^{V_{\tau}}(z)=-\frac{1}{4 \pi \tau} \sqrt{4 \tau-z^{2}} d z
$$

Prova. Por (2.19), (2.22) e (2.28) temos

$$
\begin{aligned}
\int_{\partial \mathbb{D}^{\tau}} f(z) d \widehat{\mu}^{V_{\tau}}(z) & =\frac{1}{2 \pi i\left(1-\tau^{2}\right)} \int_{\partial \mathbb{S}_{\tau}} f(z)\left(-\frac{i}{2}\left(\frac{1-\tau^{2}}{\tau}\right) \sqrt{4 \tau-z^{2}}\right) d z \\
& =-\frac{1}{4 \pi \tau} \int_{\partial \mathbb{S}^{V_{\tau}}} f(z) \sqrt{4 \tau-z^{2}} d z
\end{aligned}
$$

onde $f$ é uma função holomorfa em $\mathbb{S}_{+}^{V_{\tau}}$ e contínua em $\partial \mathbb{S}^{V_{\tau}}$. Notamos que na primeira passagem foi usado o fato de apenas o termo com a raiz quadrada na função de Schwarz ser relevante para o cálculo da integral acima pelo Teorema de Cauchy.

Por comodidade, enunciaremos o Teorema 23 novamente. 
Sejam $\mu^{V_{\tau}}$ e $\mathbb{S}^{V_{\tau}}$ dados por (2.24) e (2.25). Então

$$
\int_{\mathbb{S} V_{\tau}} f(z) d \mu^{V_{\tau}}(z)=\frac{1}{2 \pi \tau} \int_{-2 \sqrt{\tau}}^{2 \sqrt{\tau}} f(x) \sqrt{4 \tau-x^{2}} d x
$$

vale para todo $\tau \in] 0,1\left[\right.$ e toda função $f$ holomorfa em $\mathbb{S}_{+}^{V_{\tau}}$ e contínua em $\mathbb{S}^{V_{\tau}}$. Em particular, para $\tau \rightarrow 1$ temos

$$
\lim _{\tau \rightarrow 1} \mu^{V_{\tau}}=\mu_{W}
$$

onde $\mu_{W}$ é a lei do semicírculo (veja (2.27)). Além disso

$$
\lim _{\tau \rightarrow 1} V_{\tau}(x+0 i)=V_{W}(x), x \in \mathbb{R}
$$

e

$$
\lim _{\tau \rightarrow 1} V_{\tau}(z)=\infty \forall z \in \mathbb{C} \backslash \mathbb{R} .
$$

onde $V_{\tau}$ e $V_{W}$ é dado por (2.23) e (2.26) respectivamente.

Prova do Teorema 23. Pelas hipóteses sobre $f$ e pela Observação 49 item 3. temos de $(2.31)$

$$
\begin{aligned}
\int_{\mathbb{S} V_{\tau}} f(z) d \mu^{V_{\tau}}(z) & =\int_{\partial \mathbb{S}^{V_{\tau}}} f(z) d \widehat{\mu}^{V_{\tau}}(z) \\
& =-\frac{1}{4 \pi \tau} \int_{\partial \mathbb{S}_{\tau}} \sqrt{4 \tau-z^{2}} f(z) d z
\end{aligned}
$$

A função $\sqrt{4 \tau-z^{2}}$ possui um corte em $-2 \sqrt{\tau} \leq x \leq 2 \sqrt{\tau}$. Logo, pelo Teorema de Cauchy, podemos deformar a curva $\partial \mathbb{S}^{V_{\tau}}$ até que esta coincida com o corte, percorrido duas vezes: pelo lado de baixo de $-2 \sqrt{\tau}$ a $2 \sqrt{\tau}$ e pelo lado de cima de volta. Na ida, $d z=d x$ e a raiz quadrada fica $-\sqrt{4 \tau-x^{2}}$; na volta, $d z=-d x$ e a raiz quadrada fica $\sqrt{4 \tau-x^{2}}$. Logo

$$
\begin{aligned}
\int_{\mathbb{S} V_{\tau}} f(z) d \mu^{V_{\tau}}(z) & =-\frac{1}{4 \pi \tau} \int_{-2 \sqrt{\tau}}^{2 \sqrt{\tau}}(-2) \sqrt{4 \tau-x^{2}} f(x) d x \\
& =\frac{1}{2 \pi \tau} \int_{-2 \sqrt{\tau}}^{2 \sqrt{\tau}} \sqrt{4 \tau-x^{2}} f(x) d x
\end{aligned}
$$

como em (2.32). Fazendo o limite $\tau \rightarrow 1$ em (2.35) e em vista da definição de $\mu_{W}$ (2.27) encontramos (2.33). (2.34) segue do fato de $\mu_{W}$ atribuir medida nula para valores complexos não reais do domínio, logo por (2.23)

$$
\lim _{\tau \rightarrow 1} V_{\tau}(x+0 i)=\lim _{\tau \rightarrow 1} \frac{x^{2}}{1+\tau}=\frac{x^{2}}{2}=V_{W}(x)
$$


e

$$
\lim _{\tau \rightarrow 1} V_{\tau}(z)=\infty \forall z \in \mathbb{C} \backslash \mathbb{R}
$$

como queríamos.

Observação 65 A quantidade

$$
\begin{aligned}
E^{V_{\tau}} & =I\left(\mu^{V_{\tau}}\right) \\
& =\iint \log |z-w|^{-1} d \mu^{V_{\tau}}(z) \mu^{V_{\tau}}(w)+\int V(z) d \mu^{V_{\tau}}(z)
\end{aligned}
$$

é igual a $\frac{3}{4}$ para todo $\tau \in[0,1]$ e, portanto, a capacidade do suporte da medida de equilíbrio $\mu^{V_{\tau}}$ é constante como função de $\tau$ (veja definição 38). Isto significa que a energia eletrostática do sistema é fixa (a energia eletrostática está associada ao suporte da medida de equilíbrio pela definição de capacidade). Devemos contrastar esta abordagem, mantendo a energia eletrostática constante ao longo da passagem de um ensemble para outro, com a simples projeção (medida marginal) feita a seguir. Denominamos o primeiro tipo de projeção da medida de equilíbrio de projeção holomorfa.

\subsection{Outras relações entre os ensembles normal e her- mitiano: Projeção simples (medida marginal)}

Apesar deste tipo de projeção alterar a energia eletrostática, esta projeção obtém concordância com as medidas de equilíbrio de ensembles unitários normais e hermitianos mais gerais que no caso anterior.

De fato, se escolhemos o potencial $V_{H}: \Sigma=\mathbb{R} \rightarrow \mathbb{R}$

$$
V_{H}(x)=\gamma_{\alpha}|x|^{\alpha}, \alpha>0 \text { e } \gamma_{\alpha}=\frac{\Gamma\left(\frac{\alpha}{2}\right) \Gamma\left(\frac{1}{2}\right)}{\Gamma\left(\frac{\alpha+1}{2}\right)}
$$

(peso de Freud) então a medida de equilíbrio $\mu_{H}$ associada a este potencial é absolutamente contínua com respeito à medida de Lebesgue com densidade

$$
\mu_{H}^{\prime}(x)=\frac{\alpha}{\pi} \int_{|x|}^{1} \frac{u^{\alpha-1}}{\sqrt{u^{2}-x^{2}}} d u \chi_{[-1,1]}(x)
$$

(distribuição de Ullman). Para a prova deste fato veja no Apêndice D.3.

Adotando o potencial $V_{N}: \mathbb{C} \rightarrow \mathbb{R}$

$$
V_{N}(z)=\frac{2}{\alpha} r^{\alpha}, \alpha>0, z=r e^{i \theta}=x+i y,
$$


a medida de equilíbrio $\mu_{N}$ é absolutamente contínua com respeito à medida de Lebesgue em $\mathbb{C}$ com densidade

$$
d \mu_{N}(z)=\frac{\alpha}{2 \pi} r^{\alpha-1} \chi_{[0,1]}(r) d r d \theta, z=r e^{i \theta}=x+i y
$$

(veja no Apêndice D.1).

Observação 66 Integrando (2.38) na variável y mantendo $x$ constante temos, para $\alpha=2$ e em vista de (2.36), (2.37) e (2.26), (2.27)

$$
V_{H}=4 V_{W} \quad \text { e } \quad \mu_{H}(x)=\frac{1}{2} \mu_{W}\left(\frac{x}{2}\right) .
$$

Notemos que há concordância com a Seção anterior a menos do suporte da medida de equilíbrio, que é afetada pelo coeficiente do potencial (veja Observação D.5 e Corolário D.6 no Apêndice para detalhes) e

$$
V_{N}=V_{\tau} \text { e } \mu_{N}=\mu^{V_{\tau}} \text { para } \tau=0 .
$$

Prova do Teorema 24. Seja

$$
I(x) d x \equiv \int_{L_{x}} d \mu_{N}(z)
$$

onde

$$
L_{x}=\{z=x+i y \in \mathbb{D}: x \text { fixo }\} \text { e } \mathbb{D}=\{z \in \mathbb{C}:|z| \leq 1\}
$$

Escrevendo (2.38) em coordenadas cartesianas $z=x+i y$ temos

$$
\begin{aligned}
I(x) d x & =d x \int_{-\sqrt{1-x^{2}}}^{\sqrt{1-x^{2}}} \frac{\alpha}{2 \pi}\left(x^{2}+y^{2}\right)^{\frac{\alpha-2}{2}} d y \\
& =\frac{\alpha d x}{2 \pi} \int_{-\sqrt{1-|x|^{2}}}^{\sqrt{1-|x|^{2}}}\left(|x|^{2}+y^{2}\right)^{\frac{\alpha-2}{2}} d y \\
& =\frac{\alpha d x}{\pi} \int_{0}^{\sqrt{1-|x|^{2}}}\left(|x|^{2}+y^{2}\right)^{\frac{\alpha-2}{2}} d y .
\end{aligned}
$$

Fazendo a mudança de variável

$$
y(\theta)=|x| \tan \theta
$$

e definindo

$$
\theta_{1}=\arctan \left(\frac{\sqrt{1-x^{2}}}{|x|}\right)
$$


podemos escrever

$$
\begin{aligned}
I(x) d x & =\frac{\alpha d x}{\pi} \int_{0}^{\theta_{1}}\left(|x|^{2}+(|x| \tan \theta)^{2}\right)^{\frac{\alpha-2}{2}}|x| \sec ^{2} \theta d \theta \\
& =\frac{\alpha d x}{\pi} \int_{0}^{\theta_{1}}|x|^{\alpha-1} \sec ^{\alpha} \theta d \theta \\
& =\frac{\alpha d x}{\pi} \int_{0}^{\theta_{1}} \frac{(|x| \sec \theta)^{\alpha}}{|x|} d \theta .
\end{aligned}
$$

Fazendo outra mudança de variável

$$
u(\theta)=|x| \sec \theta
$$

encontramos

$$
\begin{aligned}
I(x) d x & =\frac{\alpha d x}{\pi} \int_{u(0)}^{u\left(\theta_{1}\right)} \frac{u^{\alpha}}{|x|} \frac{|x|}{u \sqrt{u^{2}-|x|^{2}}} d u \\
& =\frac{\alpha d x}{\pi} \int_{|x|}^{1} \frac{u^{\alpha-1}}{\sqrt{u^{2}-|x|^{2}}} d u \\
& =d \mu_{H}(x)
\end{aligned}
$$

e o Teorema fica provado por (2.39) e (2.37). 


\section{Capítulo 3}

\section{Núcleo integral para alguns tipos de potenciais}

\subsection{Introdução}

\subsubsection{Polinômios ortogonais}

Seja $\nu$ uma medida de Borel finita e positiva no plano complexo $\mathbb{C}$, com

$$
0<\int_{\mathbb{C}}|z|^{2 n} d \nu(z)<+\infty, \quad n=0,1,2,3, \ldots
$$

e considere o espaço de Hilbert $L^{2}(\mathbb{C}, \nu)$ formado pelas funções $f: \mathbb{C} \longrightarrow \mathbb{C}$ de quadrado integrável com respeito a $\nu$ :

$$
\int_{\mathbb{C}}|f(z)|^{2} d \nu(z)<+\infty
$$

onde duas funções são identificadas se coincidem $\nu$-quase toda parte. Assumiremos também que $\nu$ tem suporte em todo o plano $\mathbb{C}$ e $L^{2}(\mathbb{C}, \nu)$ tem dimensão infinita.

De (3.1) concluímos que todo polinômio analítico pertence a $L^{2}(\mathbb{C}, \nu)$. Denotaremos por $P^{2}(\mathbb{C}, \nu)$ o fecho do espaço vetorial dos polinômios em $L^{2}(\mathbb{C}, \nu)$ e $P_{N}^{2}(\mathbb{C}, \nu)$ o espaço vetorial separável de dimensão $N$ gerado pelos polinômios analíticos de grau $\leq N-1$ equipado com a estrutura do espaço de Hilbert $L^{2}(\mathbb{C}, \nu)$.

Os monômios

$$
e_{j}(z)=z^{j-1}, \quad j=1,2,3, \cdots, N
$$

formam uma base em $P_{N}^{2}(\mathbb{C}, \nu)$. A partir desta base podemos construir polinômios ortogonais com respeito a $\nu$ pelo procedimento de Gram-Schmidt: Seja $\varphi_{1}=e_{1}$ e para 
$k=1,2, \cdots, N$ tomamos

$$
\varphi_{k}=e_{k}+\sum_{j=1}^{k-1} a_{k, j} e_{j}
$$

onde $a_{k, j}$ são números complexos tais que

$$
\left(\varphi_{k}, e_{j}\right)_{\nu}=\int \overline{\varphi_{k}(z)} e_{j}(z) d \nu(z)=0 \quad \forall j=1, \cdots, k-1 .
$$

Notemos por (3.2) que cada $\varphi_{k}$ é um polinômio mônico, ou seja, o coeficiente de maior grau de $\varphi_{k}$ é um. Logo, nossa base ortonormal $\left\{\phi_{j}\right\}_{j=1}^{N}$ será dada por

$$
\phi_{j}=\frac{\varphi_{j}}{\left\|\varphi_{j}\right\|_{\nu}}
$$

onde

$$
\left\|\varphi_{j}\right\|_{\nu}^{2}=\int\left|\varphi_{k}(z)\right|^{2} d \nu(z)
$$

\subsubsection{Núcleo Integral}

Nesta Subseção, demonstraremos as igualdades (1.28) e (1.29).

Proposição 67 Seja

$$
P_{N}\left(z_{1}, \cdots, z_{N}\right)=Z_{N}^{-1} \prod_{1 \leq j<i \leq N}\left|z_{i}-z_{j}\right|^{2} e^{-N \sum_{i=1}^{N} V\left(z_{i}\right)}
$$

a distribuição conjunta dos autovalores de um ensemble de matrizes normais. Então $P_{N}$ pode ser escrito como

$$
P_{N}\left(z_{1}, \cdots, z_{N}\right)=C_{N} \operatorname{det}\left(K_{N}\left(z_{i}, z_{j}\right)\right)_{i, j=1}^{N}
$$

com $K_{N}$ o núcleo integral

$$
\begin{gathered}
K_{N}(z, w)=e^{-\frac{N}{2} V(z)} e^{-\frac{N}{2} \overline{V(w)}} \sum_{j=1}^{N} \phi_{j}(z) \overline{\phi_{j}(w)}, \\
C_{N}=Z_{N}^{-1} \prod_{i=1}^{N}\left\|\varphi_{i}\right\|_{\nu}^{2}
\end{gathered}
$$

$\left\{\varphi_{j}\right\}_{j=1}^{N} e\left\{\phi_{j}\right\}_{j=1}^{N}$ os polinômios ortogonais mônicos e ortonormais (respectivamente) com respeito ao peso

$$
d \nu(z)=e^{-N V(z)} d^{2} z
$$

e $V: \mathbb{C} \rightarrow \mathbb{R}$ é um potencial satisfazendo as hipóteses do Teorema 40. 
Prova. Podemos escrever $\prod_{1 \leq j<i \leq N}\left(z_{i}-z_{j}\right)$ como o determinante da matriz de Vandermonde

$$
\prod_{1 \leq j<i \leq N}\left(z_{i}-z_{j}\right)=\operatorname{det}\left(\begin{array}{ccc}
1 & \cdots & 1 \\
z_{1} & \cdots & z_{N} \\
\vdots & \ddots & \vdots \\
z_{1}^{N-1} & \cdots & z_{N}^{N-1}
\end{array}\right) \equiv \operatorname{det}\left(z_{j}^{i-1}\right)_{i, j=1}^{N}
$$

para simplificarmos a notação. Como o determinante de uma matriz é invariante por combinações lineares dos vetores que formam suas linhas, temos

$$
\begin{aligned}
\prod_{1 \leq j<i \leq N}\left(z_{i}-z_{j}\right) & =\operatorname{det}\left(\begin{array}{ccc}
\varphi_{1}\left(z_{1}\right) & \cdots & \varphi_{1}\left(z_{N}\right) \\
\varphi_{2}\left(z_{1}\right) & \cdots & \varphi_{2}\left(z_{N}\right) \\
\vdots & \ddots & \vdots \\
\varphi_{N}\left(z_{1}\right) & \cdots & \varphi_{N}\left(z_{N}\right)
\end{array}\right) \\
& =\operatorname{det}\left(\varphi_{i}\left(z_{j}\right)\right)_{i, j=1}^{N}=\prod_{i=1}^{N}\left\|\varphi_{i}\right\|_{\nu} \operatorname{det}\left(\phi_{i}\left(z_{j}\right)\right)_{i, j=1}^{N}
\end{aligned}
$$

onde $\varphi_{k}$ são polinômios mônicos (3.2), ortogonais com respeito à medida

$$
d \nu(z)=e^{-N V(z)} d^{2} z
$$

e na última passagem foi usada a multilinearidade do determinante para trocarmos os polinômios mônicos $\left\{\varphi_{k}\right\}_{k=1}^{N}$ pelos polinômios ortonormais $\left\{\phi_{k}\right\}_{k=1}^{N}$. Logo, definindo

$$
C_{N}=Z_{N}^{-1} \prod_{i=1}^{N}\left\|\varphi_{i}\right\|_{\nu}^{2}
$$

e usando (3.3) temos que

$$
\begin{aligned}
& P_{N}=Z_{N}^{-1} \prod_{1 \leq j<i \leq N}\left|z_{i}-z_{j}\right|^{2} e^{-N \sum_{i=1}^{N} V\left(z_{i}\right)} \\
& =Z_{N}^{-1} \prod_{i=1}^{N}\left\|\varphi_{i}\right\|_{\nu}^{2} \overline{\operatorname{det}\left(\phi_{i}\left(z_{j}\right)\right)_{i, j=1}^{N}} \operatorname{det}\left(\phi_{i}\left(z_{j}\right)\right)_{i, j=1}^{N} e^{-N \sum_{i=1}^{N} V\left(z_{i}\right)} \\
& =C_{N} \overline{\operatorname{det}\left(\phi_{i}\left(z_{j}\right) e^{-\frac{N}{2} V\left(z_{j}\right)}\right)_{i, j=1}^{N}} \operatorname{det}\left(\phi_{i}\left(z_{j}\right) e^{-\frac{N}{2} V\left(z_{j}\right)}\right)_{i, j=1}^{N}
\end{aligned}
$$

onde na última passagem usamos a multilinearidade do determinante e o fato de $V(z)$ ser real. Definindo a matriz de ordem $N$

$$
A=\left(\phi_{i}\left(z_{j}\right) e^{-\frac{N}{2} V\left(z_{j}\right)}\right)_{i, j=1}^{N}
$$


e usando

$$
\operatorname{det} A \overline{\operatorname{det} A}=\operatorname{det} A \overline{\operatorname{det} A^{t}}=\operatorname{det} A \operatorname{det} A^{*}=\operatorname{det} A A^{*}
$$

temos

$$
\begin{aligned}
P_{N} & =C_{N} \operatorname{det}\left(A A^{*}\right)=C_{N} \operatorname{det}\left(\sum_{k} A_{k, i} \overline{A_{k, j}}\right)_{i, j=1}^{N} \\
& =C_{N} \operatorname{det}\left(\sum_{k} \phi_{k}\left(z_{i}\right) e^{-\frac{N}{2} V\left(z_{i}\right)} \overline{\phi_{k}\left(z_{j}\right) e^{-\frac{N}{2} V\left(z_{j}\right)}}\right)_{i, j=1}^{N} \\
& =C_{N} \operatorname{det}\left(K_{N}\left(z_{i}, z_{j}\right)\right)_{i, j=1}^{N}
\end{aligned}
$$

concluindo a demonstração da proposição.

Proposição 68 A constante

$$
C_{N}=Z_{N}^{-1} \prod_{i=1}^{N}\left\|\varphi_{i}\right\|_{\nu}^{2}
$$

da Proposição anterior é dada por

$$
C_{N}=\frac{1}{N !}
$$

independente do potencial $V$.

Prova. Notamos que $K_{N}(z, w)$ dado por (3.4) possui as seguintes propriedades:

P1.

$$
\int K_{N}(z, z) d^{2} z=\int e^{-N V(z)} \sum_{j=1}^{N}\left|\phi_{j}(z)\right|^{2} d^{2} z=\sum_{j=1}^{N} 1=N
$$

Devido ao conjunto $\left\{\phi_{j}\right\}_{j=1}^{N}$ ser ortonormal com respeito ao peso $e^{-N V(z)} d^{2} z$.

P2. Também da ortonormalidade do conjunto $\left\{\phi_{j}\right\}_{j=1}^{N}$ temos

$$
\begin{aligned}
& \int \overline{K_{N}(w, z)} K_{N}(w, u) d^{2} w \\
= & e^{-\frac{N}{2} V(z)} e^{-\frac{N}{2} \overline{V(u)}} \sum_{i, j=1}^{N} \phi_{j}(z) \overline{\phi_{i}(u)} \overbrace{\int \overline{\phi_{j}(w)} \phi_{i}(w) e^{-N V(w)} d^{2} w}^{\delta_{i, j}} \\
= & e^{-\frac{N}{2} V(z)} e^{-\frac{N}{2} \overline{V(u)}} \sum_{i=1}^{N} \phi_{i}(z) \overline{\phi_{i}(u)}=K_{N}(z, u) .
\end{aligned}
$$


P1. e P2. implicam:

$$
\begin{aligned}
& \int \operatorname{det}\left(K_{N}\left(z_{i}, z_{j}\right)\right)_{i, j=1}^{N} d^{2} z_{N} \\
= & \sum_{\sigma} \operatorname{sgn}(\sigma) \int \prod_{i=1}^{N} K_{N}\left(z_{i}, z_{\sigma(i)}\right) d^{2} z_{N} \\
= & \sum_{k=1}^{N} \sum_{\sigma: \sigma(N)=k} \operatorname{sgn}(\sigma) \int \prod_{i=1}^{N} K_{N}\left(z_{i}, z_{\sigma(i)}\right) d^{2} z_{N}
\end{aligned}
$$

onde a soma acima é sobre as permutações

$$
\sigma:=\left(\begin{array}{cccc}
1, & 2, & \cdots, & N \\
\sigma(1), & \sigma(2), & \cdots, & \sigma(N)
\end{array}\right)
$$

de $(1,2, \cdots, N)$ e $\operatorname{sgn}(\sigma)$ seu sinal. Para $\sigma(N)=N$ temos, da propriedade P1:

$$
\begin{aligned}
& \sum_{\sigma: \sigma(N)=N} \operatorname{sgn}(\sigma) \int \prod_{i=1}^{N-1} K_{N}\left(z_{i}, z_{\sigma(i)}\right) K_{N}\left(z_{N}, z_{\sigma(N)}\right) d^{2} z_{N} \\
= & N \sum_{\sigma: \sigma(N)=N} \operatorname{sgn}(\sigma) \prod_{i=1}^{N-1} K_{N}\left(z_{i}, z_{\sigma(i)}\right) \\
= & N \operatorname{det}\left(K_{N}\left(z_{i}, z_{j}\right)\right)_{i, j=1}^{N-1} .
\end{aligned}
$$

Agora, para $\sigma(N)=k<N$, então existe $j: 1 \leq j \leq N$ tal que $\sigma(j)=N$. Logo, para este $\sigma$, com $i \in\{1, \cdots, N-1\} \backslash\{j\}$ e pela propriedade P2

$$
\begin{aligned}
& \int \prod_{i} K_{N}\left(z_{i}, z_{\sigma(i)}\right) K_{N}\left(z_{j}, z_{\sigma(j)}\right) K_{N}\left(z_{N}, z_{\sigma(N)}\right) d^{2} z_{N} \\
= & \prod_{i} K_{N}\left(z_{i}, z_{\sigma(i)}\right) \int K_{N}\left(z_{j}, z_{N}\right) K_{N}\left(z_{N}, z_{k}\right) d^{2} z_{N} \\
= & \prod_{i} K_{N}\left(z_{i}, z_{\sigma(i)}\right) K_{N}\left(z_{j}, z_{k}\right) \\
= & \prod_{k=1}^{N-1} K_{N}\left(z_{k}, z_{\widehat{\sigma}(k)}\right)
\end{aligned}
$$

onde $\widehat{\sigma}$ é uma permutação de $N-1$ elementos definida através da permutação $\sigma$ anterior da seguinte forma:

$$
\widehat{\sigma}:\left(\begin{array}{cccccc}
1, & 2 & , \cdots, & j & , \cdots, & N-1 \\
\sigma(1), & \sigma(2) & , \cdots, & k & , \cdots, & \sigma(N-1)
\end{array}\right)
$$


notamos que

$$
\begin{aligned}
& \operatorname{sgn}(\widehat{\sigma})=\operatorname{sgn}\left(\begin{array}{cccccc}
1, & 2 & , \cdots, & j & , \cdots, & N-1 \\
\sigma(1), & \sigma(2) & , \cdots, & k & , \cdots, & \sigma(N-1)
\end{array}\right) \\
& =\operatorname{sgn}\left(\begin{array}{cccccc}
1, & , \cdots, & j & , \cdots, & N-1 & N \\
\sigma(1), & , \cdots, & k & , \cdots, & \sigma(N-1) & N
\end{array}\right) \\
& =-\operatorname{sgn}\left(\begin{array}{cccccc}
1, & , \cdots, & j & , \cdots, & N-1 & N \\
\sigma(1), & , \cdots, & N & , \cdots, & \sigma(N-1) & k
\end{array}\right) \\
& =-\operatorname{sgn}\left(\begin{array}{cccccc}
1, & , \cdots, & j & , \cdots, & N-1 & N \\
\sigma(1), & , \cdots, & \sigma(j) & , \cdots, & \sigma(N-1) & \sigma(N)
\end{array}\right) \\
& =-\operatorname{sgn}(\sigma)
\end{aligned}
$$

Logo

$$
\begin{aligned}
& \int \operatorname{det}\left(K_{N}\left(z_{i}, z_{j}\right)\right)_{i, j=1}^{N} d^{2} z_{N} \\
= & \sum_{k=1}^{N} \sum_{\sigma: \sigma(N)=k} \operatorname{sgn}(\sigma) \int \prod_{i=1}^{N} K_{N}\left(z_{i}, z_{\sigma(i)}\right) d^{2} z_{N} \\
= & N \operatorname{det}\left(K_{N}\left(z_{i}, z_{j}\right)\right)_{i, j=1}^{N-1}-\sum_{k=1}^{N-1} \sum_{\sigma: \sigma(N)=k} \operatorname{sgn}(\widehat{\sigma}) \prod_{i=1}^{N-1} K_{N}\left(z_{i}, z_{\widehat{\sigma}(i)}\right)
\end{aligned}
$$

mas

$$
\begin{aligned}
& \sum_{k=1}^{N-1} \sum_{\sigma: \sigma(N)=k} \operatorname{sgn}(\widehat{\sigma}) \prod_{i=1}^{N-1} K_{N}\left(z_{i}, z_{\widehat{\sigma}(i)}\right) \\
= & \sum_{k=1}^{N-1} \sum_{j=1}^{N-1} \sum_{\sigma: \sigma(N)=k, \sigma(j)=k} \operatorname{sgn}(\widehat{\sigma}) \prod_{i=1}^{N-1} K_{N}\left(z_{i}, z_{\widehat{\sigma}(i)}\right) \\
= & \sum_{j=1}^{N-1} \sum_{k=1}^{N-1} \sum_{\widehat{\sigma}: \widehat{\sigma}(j)=k} \operatorname{sgn}(\widehat{\sigma}) \prod_{i=1}^{N-1} K_{N}\left(z_{i}, z_{\sigma(i)}\right) \\
= & \sum_{j=1}^{N-1} \sum_{\widehat{\sigma}} \operatorname{sgn}(\widehat{\sigma}) \prod_{i=1}^{N-1} K_{N}\left(z_{i}, z_{\sigma(i)}\right) \\
= & (N-1) \operatorname{det}\left(K_{N}\left(z_{i}, z_{j}\right)\right)_{i, j=1}^{N-1}
\end{aligned}
$$


de onde se conclui

$$
\begin{aligned}
& \int \operatorname{det}\left(K_{N}\left(z_{i}, z_{j}\right)\right)_{i, j=1}^{N} d^{2} z_{N} \\
= & N \operatorname{det}\left(K_{N}\left(z_{i}, z_{j}\right)\right)_{i, j=1}^{N-1}-(N-1) \operatorname{det}\left(K_{N}\left(z_{i}, z_{j}\right)\right)_{i, j=1}^{N-1} \\
= & (N-(N-1)) \operatorname{det}\left(K_{N}\left(z_{i}, z_{j}\right)\right)_{i, j=1}^{N-1} \\
= & \operatorname{det}\left(K_{N}\left(z_{i}, z_{j}\right)\right)_{i, j=1}^{N-1} .
\end{aligned}
$$

Notamos que o primeiro $N$ que aparece em (3.5) vem da propriedade P2 (que depende da dimensão $N$ do núcleo integral), enquanto o número $N-1$ de (3.5) vem da ordem da matriz do determinante do núcleo integral (menos um). Calculando a integral dupla notamos que

$$
\begin{aligned}
& \iint \operatorname{det}\left(K_{N}\left(z_{i}, z_{j}\right)\right)_{i, j=1}^{N} d^{2} z_{N} d^{2} z_{N-1} \\
= & (N-(N-1)) \int \operatorname{det}\left(K_{N}\left(z_{i}, z_{j}\right)\right)_{i, j=1}^{N-1} d^{2} z_{N-1} \\
= & \int \operatorname{det}\left(K_{N}\left(z_{i}, z_{j}\right)\right)_{i, j=1}^{N-1} d^{2} z_{N-1} \\
= & (N-(N-2)) \operatorname{det}\left(K_{N}\left(z_{i}, z_{j}\right)\right)_{i, j=1}^{N-2}=2 \operatorname{det}\left(K_{N}\left(z_{i}, z_{j}\right)\right)_{i, j=1}^{N-2}
\end{aligned}
$$

e a Proposição é obtida por indução finita.

Reunindo as proposições acima ficam demonstradas as igualdades (1.28) e (1.29) enunciadas na Introdução.

\subsubsection{Função de correlação de n-pontos}

Definição 69 Se $P_{N}$ é a distribuição conjunta dos autovalores de um ensemble de matrizes normais, então a função

$$
R_{n}^{N}\left(z_{1}, \cdots, z_{n}\right)=\frac{N !}{(N-n) !} \int P_{N}\left(z_{1}, \cdots, z_{N}\right) \prod_{i=n+1}^{N} d^{2} z_{i}
$$

é a função correlação de $n$ pontos associada à $P_{N}$. 
Teorema 70 Seja $R_{n}^{N}$ como acima. Então

$$
R_{n}^{N}\left(z_{1}, \cdots, z_{n}\right)=\operatorname{det}\left(K_{N}\left(z_{i}, z_{j}\right)\right)_{i, j=1}^{n}
$$

onde $K_{N}$ é o núcleo integral definido por (3.4).

Prova. Por definição e pelas Proposições 67 e 68, temos

$$
\begin{aligned}
R_{n}^{N}\left(z_{1}, \cdots, z_{n}\right) & =\frac{N !}{(N-n) !} \int P_{N}\left(z_{1}, \cdots, z_{N}\right) \prod_{i=n+1}^{N} d^{2} z_{i} \\
& =\frac{1}{(N-n) !} \int \operatorname{det}\left(K_{N}\left(z_{i}, z_{j}\right)\right)_{i, j=1}^{N} \prod_{i=n+1}^{N} d^{2} z_{i, j=1}^{N} \\
& =\operatorname{det}\left(K_{N}\left(z_{i}, z_{j}\right)\right)_{i, j=1}^{n} .
\end{aligned}
$$

onde na última passagem usamos o desenvolvimento realizado na demonstração da Proposição 68.

O Teorema anterior mostra que, dado um ensemble de matrizes aleatórias normais, o comportamento estatístico dos autovalores (para $N$ grande) está inteiramente contido no comportamento assintótico do núcleo integral (para $N$ grande). Processos estocásticos da forma (3.7) são chamados de "Determinantal random point fields" [29].

Observação 71 Se $\rho^{V}$ é a densidade associada a uma medida de equilíbrio $\mu^{V}$ absolutamente contínua com respeito à medida de Lebesgue em $\mathbb{C}$, então

$$
\rho^{V}(z)=\lim _{N \rightarrow \infty} \frac{1}{N} R_{1}^{N}(z)=\lim _{N \rightarrow \infty} \frac{1}{N} K_{N}(z, z) .
$$

A prova deste fato para o ensemble unitário hermitiano pode ser encontrada em [7] (Capítulo 6) e para o ensemble unitário normal em [27].

\subsection{Potenciais radialmente simétricos}

Prova do Teorema 25. Da relação de ortogonalidade

$$
\begin{aligned}
\left(z^{k}, z^{j}\right)_{\nu} & =\int \overline{z^{k}} z^{j} e^{-N V(z)} d^{2} z \\
& =\int_{0}^{\infty} r^{k+j} e^{-N V_{C}(r)} r d r \int_{0}^{2 \pi} e^{i \theta(j-k)} d \theta \\
& =2 \pi \delta_{k, j} \int_{0}^{\infty} r^{2 k+1} e^{-N V_{C}(r)} d r
\end{aligned}
$$


concluímos que

é um monômio. Conseqüentemente

$$
\phi_{j}(z)=\frac{z^{j-1}}{\sqrt{2 \pi \int_{0}^{\infty} r^{2 j-1} e^{-N V_{C}(r)} d r}}
$$

$$
\begin{aligned}
K_{N}(z, w) & =e^{-\frac{N}{2} V(z)} e^{-\frac{N}{2} \overline{V(w)}} \sum_{j=1}^{N} \phi_{j}(z) \overline{\phi_{j}(w)} \\
& =e^{-\frac{N}{2} V_{C}(|z|)} e^{-\frac{N}{2} \overline{V_{C}(|w|)}} \sum_{j=1}^{N} \frac{(z \bar{w})^{j-1}}{2 \pi \int_{0}^{\infty} x^{2 j-1} e^{-N V_{C}(x)} d x}
\end{aligned}
$$

como queríamos.

\subsubsection{Potenciais do tipo $V_{\alpha}(z)=|z|^{\alpha}$}

Prova do Corolário 26. Como

$$
V_{\alpha}(z)=|z|^{\alpha}, \alpha>0
$$

é um potencial que se enquadra nas hipóteses do Teorema anterior, basta calcular a quantidade:

Fazendo a mudança de variável

$$
I_{j}=\int_{0}^{\infty} r^{2 j-1} e^{-N V_{\alpha}(r)} d r=\int_{0}^{\infty} r^{2 j-1} e^{-N r^{\alpha}} d r .
$$

$$
t=N r^{\alpha} \Longrightarrow d t=\alpha N r^{\alpha-1} d r \Longrightarrow d r=\frac{1}{\alpha N}\left(\frac{t}{N}\right)^{\frac{1-\alpha}{\alpha}} d t
$$

temos

$$
\begin{aligned}
I_{j} & =\int_{0}^{\infty}\left(\frac{t}{N}\right)^{\frac{2 j-1}{\alpha}} e^{-t} \frac{1}{\alpha N}\left(\frac{t}{N}\right)^{\frac{1-\alpha}{\alpha}} d t \\
& =\frac{1}{\alpha N^{\frac{2 j}{\alpha}}} \int_{0}^{\infty}(t)^{\frac{2 j}{\alpha}-1} e^{-t} d t=\frac{1}{\alpha N^{\frac{2 j}{\alpha}}} \Gamma\left(\frac{2 j}{\alpha}\right)
\end{aligned}
$$

e

$$
\phi_{j}^{\alpha}(z)=\frac{z^{j-1}}{\sqrt{2 \pi I}}=\frac{z^{j-1}}{\sqrt{2 \pi \frac{1}{\alpha N^{\frac{2 j}{\alpha}}} \Gamma\left(\frac{2 j}{\alpha}\right)}}=\frac{N^{\frac{j}{\alpha}} z^{j-1}}{\sqrt{\frac{2 \pi}{\alpha} \Gamma\left(\frac{2 j}{\alpha}\right)}}
$$

como queríamos. Logo

$$
\begin{aligned}
K_{N}^{\alpha}(z, w) & =e^{-\frac{N}{2} V(z)} e^{-\frac{N}{2} \overline{V(w)}} \sum_{j=1}^{N} \phi_{j}^{\alpha}(z) \overline{\phi_{j}^{\alpha}(w)} \\
& =\frac{\alpha e^{-\frac{N}{2}|z|^{\alpha}} e^{-\frac{N}{2}|w|^{\alpha}}}{2 \pi} \sum_{j=1}^{N} \frac{N^{\frac{2 j}{\alpha}}(z \bar{w})^{j-1}}{\Gamma\left(\frac{2 j}{\alpha}\right)}
\end{aligned}
$$


Comportamento assintótico para $V_{\alpha}(z)=|z|^{\alpha}$.

Nosso objetivo nesta Seção é provar o Teorema 27 sobre o comportamento assintótico do núcleo integral (3.10) associado à família de potenciais $V_{\alpha}$. Para isso, alguns resultados preliminares são necessários.

Lema 72 Considere a função

$$
g(x)=\frac{\left(N^{\frac{2 \delta}{\alpha}} c\right)^{x}}{\Gamma\left(\frac{2}{\alpha} x\right)}
$$

onde $0<\delta<1, \alpha \geq 1, c \in \mathbb{C} \backslash\{0\}$, e $x \in] 0, N[$. Então, para $N$ suficientemente grande tal que $N>N_{0}=N_{0}(\alpha, \delta, c)$

$$
N_{0}=\max \left(\left(\frac{k}{|c|}\right)^{\frac{\alpha}{2 \delta}},\left(\frac{\alpha}{2}|c|^{\frac{\alpha}{2}}\right)^{\frac{1}{1-\delta}}\right)
$$

com $k>>1$ uma constante universal, existe $m \in] 0, N[$ dado por

$$
m=\frac{\alpha}{2} N^{\delta}|c|^{\frac{\alpha}{2}}-\frac{\alpha}{4}+O\left(\frac{1}{N^{\delta}}\right)
$$

tal que

$$
|g(x)| \leq|g(m)|=\frac{|c|^{\frac{\alpha}{4}}}{\sqrt{2 \pi}} N^{\frac{\delta}{2}} e^{N^{\delta}|c|^{\frac{\alpha}{2}}}\left(1+O\left(\frac{1}{N^{\delta}}\right)\right) .
$$

Prova. Como

$$
|g(x)|=\frac{\left(N^{\frac{2 \delta}{\alpha}}|c|\right)^{x}}{\Gamma\left(\frac{2}{\alpha} x\right)}
$$

temos

$$
\begin{aligned}
|g(x)|^{\prime} & =\frac{\left(N^{\frac{2 \delta}{\alpha}}|c|\right)^{x} \log \left(N^{\frac{2 \delta}{\alpha}}|c|\right) \Gamma\left(\frac{2}{\alpha} x\right)-\left(N^{\frac{2 \delta}{\alpha}}|c|\right)^{x} \frac{2}{\alpha} \Gamma^{\prime}\left(\frac{2}{\alpha} x\right)}{\left(\Gamma\left(\frac{2}{\alpha} x\right)\right)^{2}} \\
& =|g(x)|\left(\log \left(N^{\frac{2 \delta}{\alpha}}|c|\right)-\frac{2}{\alpha} \digamma\left(\frac{2}{\alpha} x\right)\right)
\end{aligned}
$$

onde

$$
\digamma(x)=\frac{\Gamma^{\prime}(x)}{\Gamma(x)}
$$

é a função digama. Como a função $|g(x)|$ não possui zeros em $] 0, N[$ e a função digama é monótona, o único ponto de máximo $x=m$ satisfaz

$$
\frac{\alpha}{2} \log \left(N^{\frac{2 \delta}{\alpha}}|c|\right)-\digamma\left(\frac{2}{\alpha} m\right)=0
$$


Para $N$ suficientemente grande $\left(N^{\frac{2 \delta}{\alpha}}|c|>k>>1\right)$ o máximo é atingido para valores aos quais a seguinte expansão assintótica [30] da função digama pode ser empregada:

$$
\digamma(x) \sim \log x+\frac{1}{2 x}-\sum_{j=1}^{\infty} \frac{B_{2 j}}{2 j x^{2 j}}
$$

onde $B_{n}$ são os números de Bernoulli

$$
\frac{t}{e^{t}-1}=\sum_{j=0}^{\infty} B_{j} \frac{t^{j}}{j !}
$$

Portanto,

$$
\log \left(N^{\delta}|c|^{\frac{\alpha}{2}}\right)=\log \frac{2}{\alpha} m+\frac{\alpha}{4 m}+O\left(\frac{1}{m^{2}}\right)
$$

ou, equivalentemente,

$$
\frac{\alpha N^{\delta}|c|^{\frac{\alpha}{2}}}{2 m}=e^{\frac{\alpha}{4 m}+O\left(\frac{1}{m^{2}}\right)}=1+\frac{\alpha}{4 m}+O\left(\frac{1}{m^{2}}\right)
$$

e assim obtemos (3.13). Para (3.12) basta resolvermos a equação (3.16) para

$$
m=N=N_{0}
$$

com $0<\delta<1$. Para (3.14), basta substituir (3.13) em $|g(m)|$ :

$$
|g(m)|=\frac{\left(N^{\frac{2 \delta}{\alpha}}|c|\right)^{m}}{\Gamma\left(\frac{2}{\alpha} m\right)}
$$

e como $m$ é ordem $N^{\delta}$ e, portanto grande, podemos usar a fórmula de Stirling em $\Gamma$ e escrever

$$
\begin{aligned}
|g(m)| & =\frac{\left(N^{\frac{2 \delta}{\alpha}}|c|\right)^{m}}{\Gamma\left(\frac{2}{\alpha} m\right)} \\
& =\sqrt{\frac{m}{\alpha \pi}}\left(\frac{\alpha e}{2 m}\right)^{\frac{2}{\alpha} m}\left(N^{\frac{2 \delta}{\alpha}}|c|\right)^{m}\left(1+O\left(\frac{1}{m}\right)\right) \\
& =\sqrt{\frac{m}{\alpha \pi}}\left(\frac{e N^{\delta}|c|^{\frac{\alpha}{2}}}{\frac{2}{\alpha} m}\right)^{\frac{2}{\alpha} m}\left(1+O\left(\frac{1}{m}\right)\right) \\
& =\sqrt{\frac{N^{\delta}}{2 \pi}}\left(\frac{\left.e\right|^{\frac{\alpha}{2}}}{N^{\delta}|c|^{\frac{\alpha}{2}}}\right)^{\frac{\alpha}{2}|c|^{\frac{\alpha}{2}}}\left(1+O\left(\frac{1}{N^{\delta}}\right)\right) \\
& =\frac{|c|^{\frac{\alpha}{4}}}{\sqrt{2 \pi}} N^{\frac{\delta}{2}} e^{N^{\delta}|c|^{\frac{\alpha}{2}}}\left(1+O\left(\frac{1}{N^{\delta}}\right)\right)
\end{aligned}
$$


como queríamos. Na penúltima passagem usamos (3.13) escrito como

$$
m=\frac{\alpha}{2} N^{\delta}|c|^{\frac{\alpha}{2}}\left(1+O\left(\frac{1}{N^{\delta}}\right)\right) .
$$

Lema 73 Seja $x$ um ponto que dista ao menos $N^{\frac{\delta}{2}} \log N$ do máximo global (3.18) da função $|g(x)|$ do Lema anterior, isto é,

$$
|x-m| \geq N^{\frac{\delta}{2}} \log N
$$

Então

$$
|g(x)| \leq\left|g\left(m \mp N^{\frac{\delta}{2}} \log N\right)\right|=\frac{|g(m)|}{N^{\frac{4 \log N}{\alpha^{2}|c|^{\frac{\alpha}{2}}}}}\left(1+O\left(\frac{\log N}{N^{\frac{\delta}{2}}}\right)\right) .
$$

Prova. Pela unicidade do ponto de máximo, concluímos que

$$
|g(x)| \leq\left|g\left(m \mp N^{\frac{\delta}{2}} \log N\right)\right| .
$$

Assim, de (3.17)

$$
\begin{aligned}
\left|g\left(m \mp N^{\frac{\delta}{2}} \log N\right)\right|= & \sqrt{\frac{m \mp N^{\frac{\delta}{2}} \log N}{\alpha \pi}}\left(\frac{e N^{\delta}|c|^{\frac{\alpha}{2}}}{\frac{2}{\alpha}\left(m \mp N^{\frac{\delta}{2}} \log N\right)}\right)^{\frac{2}{\alpha}\left(m \mp N^{\frac{\delta}{2}} \log N\right)} \\
& \cdot\left(1+O\left(\frac{1}{m}\right)\right)
\end{aligned}
$$

e, portanto, basta estimarmos os termos que aparecem na expressão assintótica (3.19):

$$
\begin{aligned}
\sqrt{\frac{m \mp N^{\frac{\delta}{2}} \log N}{\alpha \pi}} & =\sqrt{\frac{\frac{\alpha}{2} N^{\delta}|c|^{\frac{\alpha}{2}} \mp N^{\frac{\delta}{2}} \log N}{\alpha \pi}}\left(1+O\left(\frac{1}{N^{\delta}}\right)\right) \\
& =\sqrt{\frac{N^{\delta}|c|^{\frac{\alpha}{2}}}{2 \pi}} \sqrt{1 \mp \frac{2 \log N}{\alpha|c|^{\frac{\alpha}{2}} N^{\frac{\delta}{2}}}}\left(1+O\left(\frac{1}{N^{\delta}}\right)\right) \\
& =\sqrt{\frac{N^{\delta}|c|^{\frac{\alpha}{2}}}{2 \pi}}\left(1+O\left(\frac{\log N}{N^{\frac{\delta}{2}}}\right)\right) \\
\frac{2}{\alpha}\left(m \mp N^{\frac{\delta}{2}} \log N\right) & =N^{\delta}|c|^{\frac{\alpha}{2}}-\frac{1}{2}+O\left(\frac{1}{N^{\delta}}\right) \mp \frac{2}{\alpha} N^{\frac{\delta}{2}} \log N \\
& =N^{\delta}|c|^{\frac{\alpha}{2}} \mp \frac{2}{\alpha} N^{\frac{\delta}{2}} \log N-\frac{1}{2}+O\left(\frac{1}{N^{\delta}}\right)
\end{aligned}
$$




$$
\begin{aligned}
\frac{e N^{\delta}|c|^{\frac{\alpha}{2}}}{\frac{2}{\alpha}\left(m \mp N^{\frac{\delta}{2}} \log N\right)} & =\frac{e N^{\delta}|c|^{\frac{\alpha}{2}}}{N^{\delta}|c|^{\frac{\alpha}{2}}\left(1 \mp \frac{2}{\alpha|c|^{\frac{\alpha}{2}}} \frac{\log N}{N^{\frac{\delta}{2}}}-\frac{1}{2 N^{\delta}|c|^{\frac{\alpha}{2}}}+O\left(\frac{1}{N^{2 \delta}}\right)\right)} \\
& =e\left(1 \pm \frac{2}{\alpha|c|^{\frac{\alpha}{2}}} \frac{\log N}{N^{\frac{\delta}{2}}}+\frac{1}{2 N^{\delta}|c|^{\frac{\alpha}{2}}}+O\left(\frac{1}{N^{2 \delta}}\right)\right) \\
& =e^{1 \pm \frac{2}{\alpha|c|^{\frac{\alpha}{2}}} \frac{\log N}{N^{\frac{\delta}{2}}}+\frac{1}{2 N^{\delta}|c|^{\frac{\alpha}{2}}}+O\left(\frac{1}{N^{2 \delta}}\right)} .
\end{aligned}
$$

Substituindo em (3.19) resulta

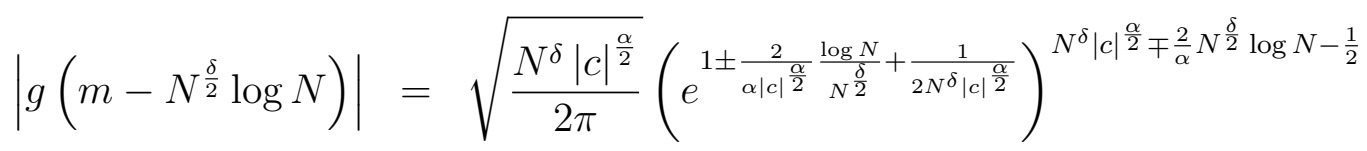

$$
\begin{aligned}
& \cdot\left(1+O\left(\frac{\log N}{N^{\frac{\delta}{2}}}\right)\right) \\
& =\sqrt{\frac{N^{\delta}|c|^{\frac{\alpha}{2}}}{2 \pi}} e^{N^{\delta}|c|^{\frac{\alpha}{2}}-\left(\frac{2}{\alpha}\right)^{2} \frac{1}{|c|^{\frac{\alpha}{2}}}(\log N)^{2}}\left(1+O\left(\frac{\log N}{N^{\frac{\delta}{2}}}\right)\right) \\
& =|g(m)| e^{-\log N^{\frac{4}{\alpha^{2}|c|^{\frac{\alpha}{2}}} \log N}}\left(1+O\left(\frac{\log N}{N^{\frac{\delta}{2}}}\right)\right) \\
& =\frac{|g(m)|}{N^{\frac{4 \log N}{\alpha^{2}|c|^{\frac{\alpha}{2}}}}}\left(1+O\left(\frac{\log N}{N^{\frac{\delta}{2}}}\right)\right)
\end{aligned}
$$

como queríamos.

Lema 74 Seja $f:[a, b] \longrightarrow \mathbb{R}$ uma função convexa:

$$
f(\lambda x+(1-\lambda) y) \leq \lambda f(x)+(1-\lambda) f(y), x, y \in[a, b] \text { e } 0<\lambda<1
$$

e

$$
P=\left\{a=x_{1}<\cdots<x_{N+2}=b\right\}
$$

uma partição uniforme de $[a, b]$ com espaçamento uniforme $\Delta x$ :

$$
x_{j}=a+(j-1) \Delta x, j \in\{1, \cdots, N+1\} .
$$

Defina $t_{j} \in\left[x_{j}, x_{j+1}\right]$ pelo Teorema do valor médio:

$$
\int_{x_{j}}^{x_{j+1}} f(x) d x=f\left(t_{j}\right) \Delta x .
$$


Então

$$
0 \geq S \geq\left(-\frac{f\left(x_{1}\right)}{2}+\frac{f\left(t_{1}\right)}{2}+\frac{f\left(x_{N}\right)}{2}-\frac{f\left(t_{N+1}\right)}{2}\right) \Delta x
$$

onde

$$
S=\sum_{j=1}^{N}\left(\int_{x_{j}}^{x_{j+1}} f(x) d x-\frac{1}{2}\left(f\left(x_{j}\right)+f\left(x_{j+1}\right)\right) \Delta x\right) .
$$

Prova. Sem perda de generalidade, suponha $f$ uma função positiva. Seja $\left\{k_{j}\right\}_{j=1}^{2 N+2}$ a seqüência numérica:

$$
k_{2 j}=\int_{x_{j}}^{x_{j+1}} f(x) d x \text { e } k_{2 j-1}=f\left(x_{j}\right) \Delta x \forall j \in\{1, \cdots, N+1\} .
$$

Pelo Teorema do valor médio, existe $t_{j} \in\left[x_{j}, x_{j+1}\right]$ tal que

$$
k_{2 j}=\int_{x_{j}}^{x_{j+1}} f(x) d x=f\left(t_{j}\right) \Delta x
$$

e por um argumento geométrico associado à convexidade da função $f$ (comparação entre áreas da figura a seguir) concluímos que a seqüência $\left\{k_{i}\right\}_{i=1}^{2 N+2}$ é tal que

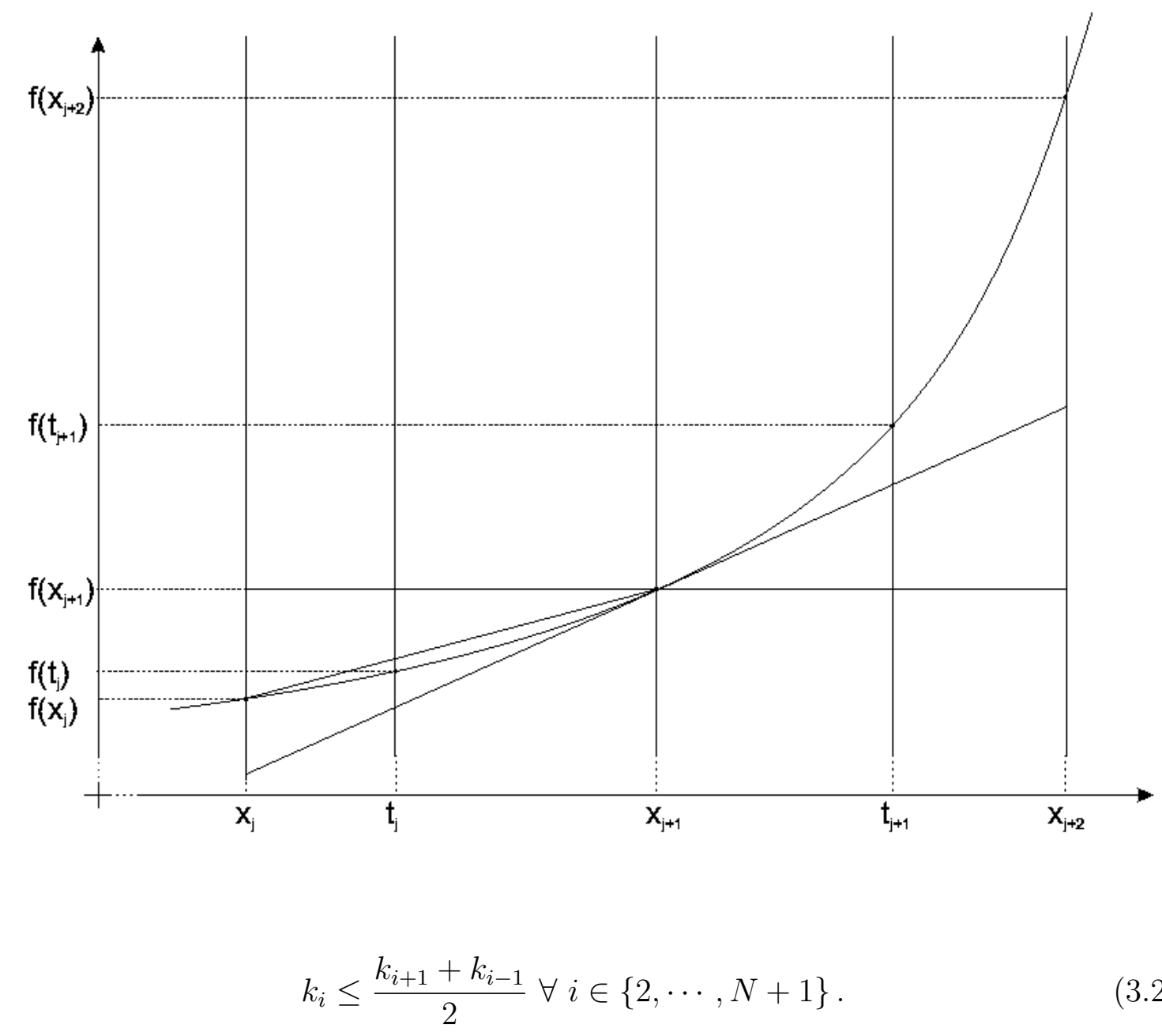


De fato, para $i=2 j$ obtemos que

$$
k_{2 j}=\int_{x_{j}}^{x_{j+1}} f(x) d x \text { e } \frac{k_{2 j+1}+k_{2 j-1}}{2}=\frac{f\left(x_{j}\right)+f\left(x_{j+1}\right)}{2} \Delta x
$$

e assim a desigualdade (3.23) é satisfeita comparando as áreas do trapézio formado pelos pontos $\left(x_{j}, 0\right),\left(x_{j+1}, 0\right),\left(x_{j}, f\left(x_{j}\right)\right)$ e $\left(x_{j+1}, f\left(x_{j+1}\right)\right)$ e sob a função $f$ no intervalo $\left[x_{j}, x_{j+1}\right]$ pois $f$ é convexa (veja figura). Para $i=2 j+1$ temos

$$
f\left(x_{j+1}\right) \Delta x=k_{2 j+1} \text { e } \frac{k_{2 j}+k_{2 j+2}}{2}=\frac{1}{2} \int_{x_{j}}^{x_{j+2}} f(t) d t
$$

e novamente a desigualdade é satisfeita comparando as áreas sob a função $f$ no intervalo $\left[x_{j}, x_{j+2}\right]$ e do retângulo de base no intervalo $\left[x_{j}, x_{j+2}\right]$ e altura $f\left(x_{j+1}\right)$. Esta última afirmação pode ser verificada se construirmos um trapézio de mesma área do retângulo anterior usando a reta tangente no ponto $\left(x_{j+1}, f\left(x_{j+1}\right)\right)$ e comparando a área deste com a área sob a função $f$ no intervalo $\left[x_{j}, x_{j+2}\right]$ (veja figura).

Consideramos a soma

$$
\begin{aligned}
S_{1} & =\sum_{j=1}^{2 N+2}(-1)^{j+1} k_{j}=k_{1}-k_{2}+\cdots+k_{l-1}-k_{2 N+2} \\
& =\frac{k_{1}}{2}-\sum_{j=1}^{N}\left(k_{2 j}-\frac{k_{2 j-1}+k_{2 j+1}}{2}\right)+\frac{k_{2 N+1}}{2}-k_{2 N+2} \\
& =k_{1}-\frac{k_{2}}{2}+\sum_{j=1}^{N}\left(k_{2 j+1}-\frac{k_{2 j}+k_{2 j+2}}{2}\right)-\frac{k_{2 N+2}}{2} .
\end{aligned}
$$

De (3.23) e (3.24) concluímos que

$$
S_{1}=\frac{k_{1}}{2}-\overbrace{\sum_{j=1}^{N}\left(k_{2 j}-\frac{k_{2 j-1}+k_{2 j+1}}{2}\right)}^{\geq 0}+\frac{k_{2 N+1}}{2}-k_{2 N+2} \geq \frac{k_{1}}{2}+\frac{k_{2 N+1}}{2}-k_{2 N+2}
$$

e de (3.23) e (3.25) concluímos que

$$
S_{1}=k_{1}-\frac{k_{2}}{2}+\overbrace{\sum_{j=1}^{\frac{l}{2}-1}\left(k_{2 j+1}-\frac{k_{2 j}+k_{2 j+2}}{2}\right)}^{\leq 0}-\frac{k_{2 N+2}}{2} \leq k_{1}-\frac{k_{2}}{2}-\frac{k_{2 N+2}}{2}
$$

o que implica que

$$
\frac{k_{1}}{2}+\frac{k_{2 N+1}}{2}-k_{2 N+2} \leq S_{1} \leq k_{1}-\frac{k_{2}}{2}-\frac{k_{2 N+2}}{2} .
$$


Pelas definições (3.21) e (3.22) podemos escrever (3.24) como

$$
S_{1}=\frac{k_{1}}{2}-S+\frac{k_{2 N+1}}{2}-k_{2 N+2}
$$

onde se conclui

$$
\frac{k_{1}}{2}+\frac{k_{2 N+1}}{2}-k_{2 N+2} \leq \frac{k_{1}}{2}-S+\frac{k_{2 N+1}}{2}-k_{2 N+2} \leq k_{1}-\frac{k_{2}}{2}-\frac{k_{2 N+2}}{2}
$$

ou, equivalentemente,

$$
0 \leq-S \leq \frac{k_{1}}{2}-\frac{k_{2}}{2}-\frac{k_{2 N+1}}{2}+\frac{k_{2 N+2}}{2}
$$

concluindo a demonstração deste Lema, em vista da definição da seqüência $\left\{k_{j}\right\}_{j=1}^{2 N+2}$.

Corolário 75 Seja $f:[a, b] \longrightarrow \mathbb{R}$ uma função côncava:

$$
f(\lambda x+(1-\lambda) y) \geq \lambda f(x)+(1-\lambda) f(y), x, y \in[a, b] \text { e } 0<\lambda<1,
$$

$P, S, \Delta x$ e $t_{j}$ como no Lema anterior. Então

$$
0 \leq S \leq\left(-\frac{f\left(x_{1}\right)}{2}+\frac{f\left(t_{1}\right)}{2}+\frac{f\left(x_{N}\right)}{2}-\frac{f\left(t_{N+1}\right)}{2}\right) \Delta x .
$$

Observação 76 Parte das idéias nesta demonstração estão contidas no argumento empregado para estabelecer o fenômeno de difração de Fresnel na referência [31] Capítulo VIII página 371.

Agora provaremos o Teorema 27.

Prova do Teorema 27. A idéia da prova é fazer uma análise assintótica em $N$ via método do ponto de sela e, portanto, assumiremos $N$ grande em relação às outras variáveis por toda esta prova.

Para esta análise, introduzimos uma escala

$$
Z=z N^{\gamma} \text { e } W=w N^{\gamma}
$$

nos argumentos do núcleo integral $K_{N}^{\alpha}$ a fim de ajustarmos uma possível dependência das variáveis $z$ e $w$ com $N$. A equação (3.10) pode ser reescrita como

$$
K_{N}^{\alpha}\left(\frac{Z}{N^{\gamma}}, \frac{W}{N^{\gamma}}\right)=\frac{\alpha e^{-\frac{N}{2}\left|\frac{Z}{N^{\gamma}}\right|^{\alpha}} e^{-\frac{N}{2}\left|\frac{W}{N^{\gamma}}\right|^{\alpha}} N^{2 \gamma}}{2 \pi Z \bar{W}} S
$$

onde

$$
S:=\sum_{j=1}^{N} \frac{\left(N^{\frac{2 \delta}{\alpha}} Z \bar{W}\right)^{j}}{\Gamma\left(\frac{2 j}{\alpha}\right)}=\sum_{j=1}^{N} \frac{\left(N^{\frac{2 \delta}{\alpha}} Z \bar{W}\right)^{t_{j} N^{\delta}}}{\Gamma\left(\frac{2 t_{j} N^{\delta}}{\alpha}\right)}
$$


onde

$$
t_{j}=\frac{j}{N^{\delta}}
$$

e

$$
\delta=1-\alpha \gamma \operatorname{com} \frac{1}{2}<\delta<1
$$

(estas escolhas serão justificadas ao longo da prova). A fórmula da soma de EulerMaclaurin ([30] página 806 para $\omega=0)$

$$
\sum_{j=0}^{N-1} f\left(y_{j}\right)=\frac{1}{h} \int_{a}^{b} f(x) d x+R_{1}+R_{2}
$$

com

$$
y_{j}=a+j h, j \in\{0, \cdots, N-1\} \text { e } y_{N-1}=b
$$

pode ser utilizada para estimar a troca de uma soma pela integral de uma função $f$ de classe $C^{(p)}$ em $[a, b]$, cujo erro é dado por

$$
R_{1}=\sum_{j=1}^{p} \frac{h^{j-1} B_{j}}{j !}\left(f^{(j-1)}(b)-f^{(j-1)}(a)\right)
$$

e

$$
R_{2}=-\frac{h^{p}}{p !} \int_{0}^{1} B_{p}(1-t)\left(\sum_{j=0}^{N-1} f^{(p)}(a+(j+t) h)\right) d t
$$

onde $B_{j}(x)$ é o $j$-ésimo polinômio de Bernoulli definidos pela série

$$
\frac{t e^{t x}}{e^{t}-1}=\sum_{j=0}^{\infty} B_{j}(x) \frac{t^{j}}{j !},|t|<2 \pi,
$$

$B_{j}(0)=B_{j}$ é o $j$-ésimo número de Bernoulli e $f^{(n)}$ é a derivada de ordem $n$ de $f$. Escolhendo

$$
\begin{gathered}
f(y)=\frac{\left(N^{\frac{2 \delta}{\alpha}} Z \bar{W}\right)^{y N^{\delta}}}{\Gamma\left(\frac{2 y N^{\delta}}{\alpha}\right)}, \\
t_{j}=N^{-\delta}+j N^{-\delta}, j \in\{0, \cdots, N-1\} \text { e } y_{N-1}=N^{1-\delta}
\end{gathered}
$$

e conseqüentemente

$$
h=N^{-\delta} \text { e }[a, b]=\left[N^{-\delta}, N^{1-\delta}\right],
$$

podemos escrever (3.28) como (escolhendo $p=1$ em (3.30))

$$
S=N^{\delta} \int_{N^{-\delta}}^{N^{1-\delta}} \frac{\left(N^{\frac{2 \delta}{\alpha}} Z \bar{W}\right)^{y N^{\delta}}}{\Gamma\left(\frac{2 y N^{\delta}}{\alpha}\right)} d y+r_{1}+r_{2}
$$


onde

$$
r_{1}=B_{1}\left(f\left(N^{1-\delta}\right)-f\left(N^{-\delta}\right)\right)=B_{1}(g(N)-g(1))
$$

e

$$
\begin{aligned}
r_{2} & =-N^{-\delta} \int_{0}^{1} B_{1}(1-t)\left(\sum_{j=0}^{N-1} f^{\prime}\left(N^{-\delta}+(j+t) N^{-\delta}\right)\right) d t \\
& =-\sum_{j=0}^{N-1} \int_{0}^{1} B_{1}(1-t) d f\left((j+t) N^{-\delta}\right) \\
& =-\sum_{j=0}^{N-1} \int_{0}^{1} B_{1}(1-t) d g(j+t) .
\end{aligned}
$$

Na última passagem de (3.34) e (3.35) usamos

$$
f\left(x N^{-\delta}\right)=g(x)=\frac{\left(N^{\frac{2 \delta}{\alpha}} Z \bar{W}\right)^{x}}{\Gamma\left(\frac{2}{\alpha} x\right)}
$$

de (3.11) e (3.32) para $c=Z \bar{W}$. Agora estimaremos $r_{1}$ e $r_{2}$.

$$
\begin{aligned}
g(N) & =\frac{\left(N^{\frac{2 \delta}{\alpha}} Z \bar{W}\right)^{N}}{\Gamma\left(\frac{2 N}{\alpha}\right)}=\sqrt{\frac{N}{\alpha \pi}}\left(\frac{\alpha e}{2 N}\right)^{\frac{2 N}{\alpha}}\left(N^{\frac{2 \delta}{\alpha}} Z \bar{W}\right)^{N} \\
& =\sqrt{\frac{1}{\alpha \pi}} \frac{\left(\left(\frac{\alpha e}{2}\right)^{\frac{2}{\alpha}} Z \bar{W}\right)^{N}}{N^{\frac{2 N}{\alpha}(1-\delta)+\frac{1}{2}}}=\frac{O\left(e^{N \log \left(\left(\frac{\alpha e}{2}\right)^{\frac{2}{\alpha}} Z \bar{W}\right)}\right)}{O\left(e^{N\left(\frac{2}{\alpha}(1-\delta)\right) \log N}\right)}=o(1)
\end{aligned}
$$

onde terceira passagem usamos a fórmula de Stirling e na última passagem usamos que $\delta<1$.

Portanto concluímos que

$$
g(1)=\frac{N^{\frac{2 \delta}{\alpha}} Z \bar{W}}{\Gamma\left(\frac{2}{\alpha}\right)}=O\left(N^{\frac{2 \delta}{\alpha}}\right)
$$

$$
r_{1}=B_{1}(g(N)-g(1))=O\left(N^{\frac{2 \delta}{\alpha}}\right) .
$$

Pelo segundo Teorema do valor médio ([33] página 107), existe $x \in[0,1]$ tal que

$$
\int_{0}^{1} B_{1}(1-t) d g(j+t)=B_{1}(1)(g(j+x)-g(j))+B_{1}(0)(g(j+1)-g(j+x))
$$

e, portanto, para $x_{j} \in[0,1](3.35)$ pode ser escrita como

$$
\begin{aligned}
r_{2} & =-\sum_{j=1}^{N}\left(B_{1}(1)\left(g\left(j+x_{j}\right)-g(j)\right)+B_{1}(0)\left(g(j+1)-g\left(j+x_{j}\right)\right)\right) \\
& =\frac{1}{2} \sum_{j=1}^{N}\left(2 g\left(j+x_{j}\right)-(g(j)+g(j+1))\right)
\end{aligned}
$$


Na última passagem usamos que

$$
B_{1}(0)=-B_{1}(1)=\frac{1}{2}
$$

Primeiramente considere $Z \bar{W}$ um número real positivo. Desta forma

$$
g(x)=|g(x)|=\frac{\left(N^{\frac{2 \delta}{\alpha}}|Z \bar{W}|\right)^{x}}{\Gamma\left(\frac{2}{\alpha} x\right)}
$$

Agora, podemos fazer uso do Lema 72 para escrever:

$r_{2}=\frac{1}{2}\left(\sum_{j=1}^{\lfloor m\rfloor}\left(2 g\left(j+x_{j}\right)-(g(j)+g(j+1))\right)+\sum_{j=\lfloor m\rfloor+1}^{N}\left(2 g\left(j+x_{j}\right)-(g(j)+g(j+1))\right)\right)$

onde $m$ é dado por (3.13),

$$
\lfloor x\rfloor=\text { maior inteiro menor ou igual a } x \text {. }
$$

Logo, como $0 \leq x_{j} \leq 1$ e $g(x)$ é crescente em $[1, m)$ e decrescente em $(m, N]$ e supondo primeiramente $r_{2} \geq 0$ podemos escrever

$$
\begin{aligned}
r_{2} & <\frac{1}{2}\left(\sum_{j=1}^{\lfloor m\rfloor}(g(j+1)-g(j))+\sum_{j=\lfloor m\rfloor+1}^{N}(g(j)-g(j+1))\right) \\
& =\frac{1}{2}(g(\lfloor m\rfloor+1)-g(1)+g(\lfloor m\rfloor+1)-g(N+1)) \\
& \leq g(m)-\frac{1}{2}(g(1)+g(N+1))=g(m)-\frac{1}{2}\left(f\left(N^{-\delta}\right)+f\left(N^{-\delta}(N+1)\right)\right) \\
& =\frac{|Z \bar{W}|^{\frac{\alpha}{4}}}{\sqrt{2 \pi}} N^{\frac{\delta}{2}} e^{N^{\delta}|Z \bar{W}|^{\frac{\alpha}{2}}}\left(1+O\left(\frac{1}{N^{\delta}}\right)\right)-O\left(N^{\frac{2 \delta}{\alpha}}\right)=O\left(N^{\frac{\delta}{2}} e^{N^{\delta}|Z \bar{W}|^{\frac{\alpha}{2}}}\right)
\end{aligned}
$$

onde na antepenúltima passagem usamos (3.36) e na penúltima passagem usamos as estimativas para $g(m)$ e $r_{1}$ ( Lema 72 e (3.37)). O caso em que $r_{2}<0$ pode ser estimado de maneira análoga. Assim obtemos

$$
\left|r_{2}\right|=O\left(N^{\frac{\delta}{2}} e^{N^{\delta}|z \bar{W}|^{\frac{\alpha}{2}}}\right)
$$

Agora considere $Z \bar{W}$ com argumento $\theta \in]-\pi, 0[\cup] 0, \pi]$. De (3.11) podemos escrever

$$
g(x)=\frac{\left(N^{\frac{2 \delta}{\alpha}} Z \bar{W}\right)^{x}}{\Gamma\left(\frac{2}{\alpha} x\right)}=\frac{\left(N^{\frac{2 \delta}{\alpha}}|Z \bar{W}| e^{i \theta}\right)^{x}}{\Gamma\left(\frac{2}{\alpha} x\right)}=|g(x)| e^{i \theta x}
$$


de forma que

$$
\operatorname{Re}(g(x))=|g(x)| \cos (\theta x) \text { e } \operatorname{Im}(g(x))=|g(x)| \operatorname{sen}(\theta x) .
$$

Basta estimar Re $(g(x))$, pois a estimativa de $\operatorname{Im}(g(x))$ pode ser feita de maneira análoga. Assim, dependendo do período

$$
p=\frac{2 \pi}{|\theta|} \geq 2
$$

teremos tantas raízes

$$
\frac{\pi}{2|\theta|} l
$$

da função $\operatorname{Re}(g(x))$ no intervalo $] 1, N[$ quantos inteiros ímpares $l \in \mathbb{Z}$ satisfazendo

$$
\frac{2|\theta|}{\pi}<l<\frac{2|\theta| N}{\pi}
$$

Assim podemos escrever (3.38) como

$$
\begin{aligned}
\operatorname{Re}\left(r_{2}\right) & =\frac{1}{2} \sum_{j=1}^{N}\left(2 \operatorname{Re}\left(g\left(j+x_{j}\right)\right)-(\operatorname{Re}(g(j))+\operatorname{Re}(g(j+1)))\right) \\
& =S_{C}+S_{D}
\end{aligned}
$$

onde

$$
S_{C(D)}=\sum_{j \in \mathbb{A}_{C(D)}}\left(\operatorname{Re}\left(g\left(j+x_{j}\right)\right)-\left(\frac{\operatorname{Re}(g(j))+\operatorname{Re}(g(j+1))}{2}\right)\right)
$$

com

$$
\mathbb{A}_{C}=\left\{j \in\{1, \cdots, N\}: \operatorname{Re}(g(j+1))-\operatorname{Re}\left(g\left(j+x_{j}\right)\right) \geq 0\right\}
$$

e

$$
\mathbb{A}_{D}=\left\{j \in\{1, \cdots, N\}: \operatorname{Re}(g(j+1))-\operatorname{Re}\left(g\left(j+x_{j}\right)\right)<0\right\}
$$

Em palavras, separamos a soma em duas regiões: a região em que os índices da soma estão entre um mínimo e um máximo e a região complementar. Notemos que como $0<|\theta| \leq \pi$ é arbitrário em (3.41), a cardinalidade ${ }^{1}$ do conjunto $\mathbb{A}_{C} \cup \mathbb{A}_{D}$ varia de 2 a $N$, com $N$ um número grande porém fixo. Para $n\left(\mathbb{A}_{C} \cup \mathbb{A}_{D}\right)=2$, a soma (3.45) recai na expressão (3.39) enquanto que se $n\left(\mathbb{A}_{C} \cup \mathbb{A}_{D}\right)=N$ a soma (3.45) é exatamente (3.44) pois neste

\footnotetext{
${ }^{1} \mathrm{~A}$ cardinalidade $n(\mathbb{A})$ de um conjunto discreto $\mathbb{A}$ é o número de elementos deste conjunto.
} 
caso a oscilação da função $\operatorname{Re}(g(x))$ é $O(1)$. Podemos estimar $S_{C}$ e $S_{D}$, primeiramente supondo ambos não negativos, da seguinte forma:

$$
\begin{aligned}
S_{C} & \leq \sum_{j \in \mathbb{A}_{C}}\left(\operatorname{Re}(g(j+1))-\left(\frac{\operatorname{Re}(g(j))+\operatorname{Re}(g(j+1))}{2}\right)\right) \\
& =\frac{1}{2} \sum_{j \in \mathbb{A}_{C}}(\operatorname{Re}(g(j+1))-\operatorname{Re}(g(j)))
\end{aligned}
$$

e

$$
\begin{aligned}
S_{D} & \leq \sum_{j \in \mathbb{A}_{D}}\left(\operatorname{Re}(g(j))-\left(\frac{\operatorname{Re}(g(j))+\operatorname{Re}(g(j+1))}{2}\right)\right) \\
& =\frac{1}{2} \sum_{j \in \mathbb{A}_{D}}(\operatorname{Re}(g(j))-\operatorname{Re}(g(j+1)))
\end{aligned}
$$

pois $0 \leq x_{j} \leq 1$ e escolhemos $x_{j}=0$ ou 1 de acordo com os conjuntos $\mathbb{A}_{C}$ ou $\mathbb{A}_{D}$, que definem a monotonicidade da função $\operatorname{Re}(g(x))$. Assim

$$
\operatorname{Re}\left(r_{2}\right) \leq \frac{1}{2}\left(\sum_{j \in \mathbb{A}_{C}}(\operatorname{Re}(g(j+1))-\operatorname{Re}(g(j)))+\sum_{j \in \mathbb{A}_{D}}(\operatorname{Re}(g(j))-\operatorname{Re}(g(j+1)))\right) .
$$

Logo, quando $n\left(\mathbb{A}_{C} \cup \mathbb{A}_{D}\right)=O(1)$ em $N$ ainda obtemos que

$$
\left|r_{2}\right|=O\left(N^{\frac{\delta}{2}} e^{N^{\delta}|Z \bar{W}|^{\frac{\alpha}{2}}}\right)
$$

pelos mesmos argumentos utilizados para a obtenção de (3.40).

Como $n\left(\mathbb{A}_{C} \cup \mathbb{A}_{D}\right)$ pode ser $O(N)$ (lembrando que isso ocorre com o aumento das oscilações da função $\operatorname{Re}(g(x))$ como função de $\theta$, devido a (3.41)), devemos estimar (3.44) de outra forma e introduzir uma escala

$$
n\left(\mathbb{A}_{C} \cup \mathbb{A}_{D}\right)=O\left(N^{\varepsilon}\right), 0<\varepsilon<1
$$

que separa uma estimativa da outra. Integrando (3.35) por partes temos

$$
\begin{aligned}
r_{2} & =\sum_{j=1}^{N} \int_{0}^{1} B_{1}(1-t) d g(j+t) \\
& =\sum_{j=1}^{N}(\left.\overbrace{\left(\frac{1}{2}-t\right)}^{B_{1}(1-t)} g(j+t)\right|_{0} ^{1}+\int_{0}^{1} g(j+t) d t) \\
& =\sum_{j=1}^{N}\left(\int_{0}^{1} g(j+t) d t-\frac{1}{2}(g(j)-g(j+1))\right) .
\end{aligned}
$$


Separando a parte real da soma acima em duas partes, temos

$$
\operatorname{Re}\left(r_{2}\right)=\sum_{j=1}^{N}\left(\int_{0}^{1} \operatorname{Re}(g(j+t)) d t-\frac{1}{2}(\operatorname{Re}(g(j))-\operatorname{Re}(g(j+1)))\right)=S_{\sqcup}+S_{\sqcap}
$$

onde

$$
S_{\sqcup(\sqcap)}=\sum_{j \in \mathbb{A}_{\sqcup(\sqcap)}}\left(\int_{0}^{1} \operatorname{Re}(g(j+t)) d t-\frac{1}{2}(\operatorname{Re}(g(j))-\operatorname{Re}(g(j+1)))\right)
$$

com

$$
\mathbb{A}_{\sqcup}=\{j \in\{1, \cdots, N\}: \operatorname{Re}(g(j)) \text { é convexa }\}
$$

e

$$
\mathbb{A}_{\sqcap}=\{j \in\{1, \cdots, N\}: \operatorname{Re}(g(j)) \text { é côncava }\} \text {. }
$$

Notemos que a função Re $(g(j))$ sempre tem concavidade bem definida (a menos dos pontos de inflexão, que são isolados) e que a cardinalidade dos conjuntos $\mathbb{A}_{\sqcup}, \mathbb{A}_{\sqcap}, \mathbb{A}_{C}$ e $\mathbb{A}_{D}$ são da mesma ordem em $N$, pois a principal função responsável tanto pelo número de raízes quanto pelas mudanças de concavidade é o cosseno na função

$$
\operatorname{Re}(g(x))=|g(x)| \cos (\theta x) .
$$

Notemos também que quando

$$
n\left(\mathbb{A}_{\sqcup} \cup \mathbb{A}_{\sqcap}\right)=O\left(N^{\varepsilon}\right), 0<\varepsilon<1,
$$

cada conjunto $\mathbb{A}_{\sqcup}$ e $\mathbb{A}_{\sqcap}$ é formado pela união de conjuntos disjuntos de números consecutivos, uma vez que a função $\operatorname{Re}(g(x))$ tem sempre convexidade bem definida:

$$
\mathbb{A}_{\sqcup}=\bigcup_{i} \mathbb{B}_{\sqcup_{i}} \text { e } \mathbb{A}_{\sqcap}=\bigcup_{i} \mathbb{B}_{\sqcap_{i}}
$$

onde cada $i, \mathbb{B}_{\sqcup_{i}}$ e $\mathbb{B}_{\Gamma_{i}}$ possuem cardinalidade de mesma ordem, tal que

$$
\left.n\left(\mathbb{B}_{\sqcup_{i}} \cup \mathbb{B}_{\sqcap_{i}}\right)=O\left(N^{\varepsilon^{\prime}}\right), \varepsilon+\varepsilon^{\prime}=1, \operatorname{com} \varepsilon, \varepsilon^{\prime} \in\right] 0,1[,
$$

novamente devido à (3.51). Notemos que nestas condições temos de (3.41) que

$$
p=\frac{2 \pi}{|\theta|}=O\left(N^{\varepsilon}\right) \Longrightarrow \theta=O\left(N^{-\varepsilon}\right)
$$

Assim, usando o Lema 74 (e conseqüentemente o Corolário 75) podemos concluir que apenas a vizinhança dos pontos de inflexão contribuem para a soma (3.50). Portanto, 
estimaremos a ordem do maior ponto de inflexão e quantos deles podem contribuir para a soma (3.50). Derivando (3.51) obtemos

$$
\operatorname{Re}(g(x))^{\prime \prime}=\left(|g(x)|^{\prime \prime}-\theta^{2}|g(x)|\right) \cos (\theta x)-2 \theta|g(x)|^{\prime} \operatorname{sen}(\theta x)
$$

Por um lado a expressão acima pode ser escrita como

$$
\operatorname{Re}(g(x))^{\prime \prime}=|g(x)|^{\prime \prime} \cos (\theta x)\left(1+O\left(N^{-\varepsilon}\right)\right)
$$

pois quando derivamos a função $|g(x)|$ ocorre no máximo um aumento logarítmico em sua ordem máxima em $N$ (veja por exemplo (3.15)). Por outro lado

$$
\operatorname{Re}\left(g\left(x_{i}\right)\right)^{\prime \prime}=\left(\left|g\left(x_{i}\right)\right|^{\prime \prime}-\theta^{2}\left|g\left(x_{i}\right)\right|\right) \cos \left(\theta x_{i}\right)-2 \theta\left|g\left(x_{i}\right)\right|^{\prime} \operatorname{sen}\left(\theta x_{i}\right)=0
$$

no ponto de inflexão $x_{i}$. Disso concluímos que o ponto de inflexão dista ordem 1 de uma raiz $\frac{\pi}{2|\theta|} l$ da função $\cos (\theta x)$ ( $l$ inteiro ímpar). Definindo $\Delta x_{i}=O(1)$ de forma que

$$
x_{i}=\frac{\pi}{2|\theta|} l+\Delta x_{i}
$$

obtemos

$$
\begin{aligned}
\cos \left(\theta x_{i}\right) & =\cos \left( \pm \frac{\pi}{2} l \pm \theta \Delta x_{i}\right)=\cos \left(\frac{\pi}{2} l\right) \cos \left(\theta \Delta x_{i}\right)-\operatorname{sen}\left( \pm \frac{\pi}{2} l\right) \operatorname{sen}\left( \pm \theta \Delta x_{i}\right) \\
& = \pm \operatorname{sen}\left(\theta \Delta x_{i}\right)=O\left(N^{-\varepsilon}\right) .
\end{aligned}
$$

Logo, a ordem do maior ponto de inflexão será

$$
\left|\operatorname{Re}\left(g\left(x_{i}\right)\right)\right| \leq O\left(N^{\frac{\delta}{2}-\varepsilon} e^{N^{\delta}|Z \bar{W}|^{\frac{\alpha}{2}}}(\log N)^{2}\right)
$$

e, portanto, a soma (3.50) pode ser estimada usando o Lema 73 e a definição de $\varepsilon$ e $\varepsilon^{\prime}$ :

$$
\begin{aligned}
\left|r_{2}\right| & \leq O\left(N^{\frac{\delta}{2}-\varepsilon} e^{N^{\delta}|Z \bar{W}|^{\frac{\alpha}{2}}}(\log N)^{2} N^{\frac{\delta}{2}-\varepsilon^{\prime}} \log N\right) \\
& =O\left(N^{\delta-1} e^{N^{\delta}|Z \bar{W}|^{\frac{\alpha}{2}}}(\log N)^{3}\right) .
\end{aligned}
$$

Basta estimarmos o caso em que temos oscilações da ordem $N$ :

$$
n\left(\mathbb{A}_{\sqcup} \cup \mathbb{A}_{\sqcap}\right)=O(N)=n\left(\mathbb{A}_{C} \cup \mathbb{A}_{D}\right)
$$


Neste caso tanto $\theta$ quanto $p$ é $O(1)$ em $N$ (veja (3.41)). De (3.35) temos

$$
\begin{aligned}
r_{2} & =\sum_{j=1}^{N} \int_{0}^{1} B_{1}(1-t) d g(j+t) \\
& =\sum_{j=1}^{N} \int_{0}^{1} \widehat{B}_{1}(1-t+j) d g(j+t)=\int_{1}^{N} \widehat{B}_{1}(1-t) d g(t) \\
& =\int_{1}^{N} \widehat{B}_{1}(1-t) d g(t)
\end{aligned}
$$

onde

$$
\widehat{B}_{1}(x)=B_{1}(x \bmod 1), B_{1}:\left[0,1\left[\rightarrow \left[-\frac{1}{2}, \frac{1}{2}[\right.\right.\right.
$$

é a extensão periódica do polinômio de Bernoulli de grau um. Com o auxilio das equações (3.51), (3.15) e pela linearidade da derivada, podemos escrever a parte real de (3.54) como

$$
\begin{aligned}
\operatorname{Re}\left(r_{2}\right) & =\int_{1}^{N} \widehat{B}_{1}(1-t)(|g(t)| \cos (\theta t))^{\prime} d t \\
& =\int_{1}^{N} \widehat{B}_{1}(1-t)\left(|g(t)|^{\prime} \cos (\theta t)+\theta|g(t)| \operatorname{sen}(\theta t)\right) d t \\
& =\int_{1}^{N} \widehat{B}_{1}(1-t)|g(t)|\left(\left(\log \left(N^{\frac{2 \delta}{\alpha}}|c|\right)-\frac{2}{\alpha} \digamma\left(\frac{2}{\alpha} t\right)\right) \cos (\theta t)+\theta \operatorname{sen}(\theta t)\right) d t \\
& \equiv N \int_{\frac{1}{N}}^{1}\left(f_{1}(s) \cos (\theta N s)+f_{2}(s) \operatorname{sen}(\theta N s)\right) d s \equiv I_{1}+I_{2}
\end{aligned}
$$

onde

$$
f_{1}(s)=\widehat{B}_{1}(1-N s)|g(N s)|\left(\log \left(N^{\frac{2 \delta}{\alpha}}|c|\right)-\frac{2}{\alpha} \digamma\left(\frac{2}{\alpha} N s\right)\right)
$$

e

$$
f_{2}(s)=\theta \widehat{B}_{1}(1-N s)|g(N s)|
$$

com

$$
t=N s, s \in\left[\frac{1}{N}, 1\right]
$$

Notemos que

$$
\max _{s \in\left[\frac{1}{N}, 1\right]}\left|f_{1}(s)\right| \leq|g(m)|\left(\log \left(N^{\frac{2 \delta}{\alpha}}|c|\right)-\frac{2}{\alpha} \digamma\left(\frac{2}{\alpha} N s\right)\right)=O\left(N^{\frac{\delta}{2}} e^{N^{\delta}|z \bar{W}|^{\frac{\alpha}{2}}} \log N\right)
$$

e

$$
\max _{s \in\left[\frac{1}{N}, 1\right]}\left|f_{2}(s)\right| \leq \theta|g(m)|=O\left(N^{\frac{\delta}{2}} e^{N^{\delta}|Z \bar{W}|^{\frac{\alpha}{2}}}\right)
$$


pelo Lema 72 e pela relação (3.57). Assim

$$
\begin{aligned}
I_{1} & =N \int_{\frac{1}{N}}^{1} f_{1}(s) \cos (\theta N s) d s \\
& =N \sum_{i=1}^{n} f_{1}\left(s_{i}\right) \int_{s_{i}}^{s_{i+1}} \cos (\theta N s) d s+N \sum_{i=1}^{n} \int_{s_{i}}^{s_{i+1}}\left(f_{1}(s)-f_{1}\left(s_{i}\right)\right) \cos (\theta N s) d s
\end{aligned}
$$

onde

$$
P=\left\{\frac{1}{N}=s_{1}<\cdots<s_{n+1}=1\right\}
$$

é uma partição com espaçamento

$$
\Delta s_{i}=s_{i+1}-s_{i}=O\left(N^{\frac{\delta}{2}-1} \log N\right) .
$$

Logo, da desigualdade

$$
\left|\int_{s_{i}}^{s_{i+1}} \cos (\theta N x) d x\right|=\left|\frac{\operatorname{sen}(\theta N s)}{\theta N}\right|_{s_{i}}^{s_{i+1}} \leq \frac{2}{\theta N},
$$

do Lema 73 e da escolha do espaçamento $\Delta s_{i}$ da partição $P$, obtemos

$$
\left|I_{1}\right| \leq N \max _{i}\left(\max _{s \in\left[s_{i}, s_{i+1}\right]}\left|f_{1}(s)\right| \frac{2}{\theta N}+\left|\int_{s_{i}}^{s_{i+1}}\left(f_{1}(s)-f_{1}\left(s_{i}\right)\right) \cos (\theta N s) d s\right|\right)
$$

pois apenas um (ou no máximo dois) dos intervalos $\left[s_{i}, s_{i+1}\right]$ da partição $P$ contém um número relevante de máximos da ordem dada pela expressão (3.58). Defina

$$
I_{3} \equiv \int_{s_{i}}^{s_{i+1}}\left(f_{1}(s)-f_{1}\left(s_{i}\right)\right) \cos (\theta N s) d s .
$$

Expandindo $f_{1}$ em série de Taylor temos

$$
f_{1}(s)-f_{1}\left(s_{i}\right)=f_{1}^{\prime}\left(s_{i}\right)\left(s-s_{i}\right)+E
$$

onde

$$
E=\frac{f_{1}^{\prime \prime}(\xi)}{2}\left(s-s_{i}\right)^{2}, \xi \in\left[s_{i}, s_{i+1}\right]
$$

Da definição da função $f_{1}$ (3.56) podemos notar que com a operação derivada, ocorre um aumento de $\log N$ na ordem do máximo $f_{1}^{\prime}$ em relação a $f_{1}$. Assim, deste fato e da equação (3.60) obtemos

$$
\begin{aligned}
\left|f_{1}^{\prime}\left(s_{i}\right)\left(s-s_{i}\right)\right| & \leq\left|f_{1}^{\prime}\left(s_{i}\right)\right|\left|s_{i+1}-s_{i}\right| \\
& =O\left(N^{\frac{\delta}{2}} e^{N^{\delta}|Z \bar{W}|^{\frac{\alpha}{2}}} \log ^{2} N\right) \cdot O\left(N^{\frac{\delta}{2}-1} \log N\right) \\
& =O\left(N^{\delta-1} e^{N^{\delta}|Z \bar{W}|^{\frac{\alpha}{2}}} \log ^{3} N\right)
\end{aligned}
$$


$\mathrm{e}$

$$
\begin{aligned}
|E| & \leq \frac{\left|f_{1}^{\prime \prime}(\xi)\right|}{2}\left|s_{i+1}-s_{i}\right|^{2} \\
& \leq O\left(N^{\frac{\delta}{2}} e^{N^{\delta}|Z \bar{W}|^{\frac{\alpha}{2}}} \log ^{3} N\right) \cdot O\left(N^{\delta-2} \log ^{2} N\right) \\
& =O\left(N^{\frac{3 \delta}{2}-2} e^{N^{\delta}|Z \bar{W}|^{\frac{\alpha}{2}}} \log ^{5} N\right) .
\end{aligned}
$$

Logo

$$
\begin{aligned}
& \left|I_{3}\right| \leq\left|f_{1}^{\prime}\left(s_{i}\right) \int_{s_{i}}^{s_{i+1}}\left(s-s_{i}\right) \cos (\theta N s) d s\right|+|E|\left|\int_{s_{i}}^{s_{i+1}}\right| \cos (\theta N s)|d s| \\
& \leq\left|f_{1}^{\prime}\left(s_{i}\right) \int_{0}^{\Delta s_{i}} t \cos \left(\theta N\left(t+s_{i}\right)\right) d t\right|+|E|\left|\Delta s_{i}\right| \\
& =\left|f_{1}^{\prime}\left(s_{i}\right)\left(\left.\frac{t}{\theta N} \operatorname{sen}\left(\theta N\left(t+s_{i}\right)\right)\right|_{0} ^{\Delta s_{i}}-\int_{0}^{\Delta s_{i}} \frac{1}{\theta N} \operatorname{sen}\left(\theta N\left(t+s_{i}\right)\right) d t\right)\right|+|E|\left|\Delta s_{i}\right| \\
& \leq\left|f_{1}^{\prime}\left(s_{i}\right)\left(\frac{\Delta s_{i}}{\theta N} \operatorname{sen}\left(\theta N\left(t+s_{i}\right)\right)+\left.\frac{1}{(\theta N)^{2}} \cos \left(\theta N\left(t+s_{i}\right)\right)\right|_{0} ^{\Delta s_{i}}\right)\right|+|E|\left|\Delta s_{i}\right| \\
& =O\left(N^{\frac{\delta}{2}} e^{N^{\delta}|Z \bar{W}|^{\frac{\alpha}{2}}} \log ^{2} N\right) \cdot O\left(N^{\frac{\delta}{2}-2} \log N\right) \\
& =O\left(N^{\delta-2} e^{N^{\delta}|Z \bar{W}|^{\frac{\alpha}{2}}} \log ^{3} N\right)
\end{aligned}
$$

onde na penúltima passagem usamos (3.62) e (3.63), pegando apenas o termo líder em $N$. Assim, substituindo (3.64) e (3.58) em (3.61) temos

$$
\begin{aligned}
\left|I_{1}\right| & =N\left(O\left(N^{\frac{\delta}{2}} e^{N^{\delta}|Z \bar{W}|^{\frac{\alpha}{2}}} \log N\right) \frac{2}{\theta N}+O\left(N^{\delta-2} e^{N^{\delta}|Z \bar{W}|^{\frac{\alpha}{2}}} \log ^{3} N\right)\right) \\
& =O\left(N^{\frac{\delta}{2}} e^{N^{\delta}|Z \bar{W}|^{\frac{\alpha}{2}}} \log N\right)
\end{aligned}
$$

pois $\delta \in] \frac{1}{2}, 1\left[\right.$. Procedendo de maneira análoga para $I_{2}$ em (3.55) obtemos

$$
\left|I_{2}\right|=O\left(N^{\frac{\delta}{2}} e^{N^{\delta}|Z \bar{W}|^{\frac{\alpha}{2}}}\right) .
$$

Finalmente, concluímos que

$$
\left|r_{2}\right|<O\left(N^{\frac{\delta}{2}} e^{N^{\delta}|Z \bar{W}|^{\frac{\alpha}{2}}} \log N\right)
$$


e, portanto, de (3.33), (3.32) e das estimativa (3.37) para $r_{1}$ e (3.65) para $r_{2}$ obtemos

$$
S=N^{\delta} \int_{N^{-\delta}}^{N^{1-\delta}} f(x) d x+R
$$

onde

$$
f(x)=\frac{\left(N^{\frac{2 \delta}{\alpha}} Z \bar{W}\right)^{x N^{\delta}}}{\Gamma\left(\frac{2 x N^{\delta}}{\alpha}\right)}
$$

e

$$
R=O\left(N^{\frac{\delta}{2}} e^{N^{\delta}|Z \bar{W}|^{\frac{\alpha}{2}}} \log N\right)+O\left(N^{\delta-1} e^{N^{\delta}|Z \bar{W}|^{\frac{\alpha}{2}}}(\log N)^{3}\right)
$$

Usando a fórmula de Stirling para o denominador de $f$ obtemos

$$
f=\Phi\left(1+O\left(\frac{1}{N^{\delta}}\right)\right)
$$

onde

$$
\begin{aligned}
& \Phi(x)=\sqrt{\frac{x N^{\delta}}{\alpha \pi}}\left(\frac{\alpha e}{2 x N^{\delta}}\right)^{\frac{2 x N^{\delta}}{\alpha}}\left(N^{\frac{2 \delta}{\alpha}} Z \bar{W}\right)^{x N^{\delta}}=\sqrt{\frac{x N^{\delta}}{\alpha \pi}}\left(\frac{\alpha e}{2 x}\right)^{\frac{2 x N^{\delta}}{\alpha}}(Z \bar{W})^{x N^{\delta}} \\
& =\sqrt{\frac{N^{\delta}}{\alpha \pi}} \sqrt{x} e^{N^{\delta} x\left(\log \left(\left(\frac{\alpha e}{2 x}\right)^{\frac{2}{\alpha}} Z \bar{W}\right)\right)}:=\sqrt{\frac{N^{\delta}}{\alpha \pi}} \sqrt{x} e^{N^{\delta} h(x)}
\end{aligned}
$$

Notemos que a simplificação feita na antepenúltima passagem da expressão acima ocorre devido a equação (3.29), que relaciona a escala $\delta$ de integração com a escala $\gamma$ das variáveis do argumento do núcleo integral $K_{N}^{\alpha}$ para que a função $h$ definida na última passagem não dependa de $N$. De (3.68) concluímos que para $x \in\left[N^{-\delta}, N^{1-\delta}\right]$,

$$
\frac{f(x)-\Phi(x)}{\Phi(x)}=O\left(\frac{1}{N^{\delta}}\right)
$$

e, portanto,

$$
\int_{N^{-\delta}}^{N^{1-\delta}}\left(\frac{f(x)-\Phi(x)}{\Phi(x)}\right) d x=O\left(\frac{N^{1-\delta}}{N^{\delta}}\right)=O\left(N^{1-2 \delta}\right) .
$$

Assim, podemos trocar $f$ por $\Phi$ em (3.66), pois $\frac{1}{2}<\delta<1$. Logo

$$
\begin{aligned}
S & =N^{\delta} \int_{N^{-\delta}}^{N^{1-\delta}} \Phi(x) d x\left(1+O\left(N^{1-2 \delta}\right)\right)+R \\
& =\sqrt{\frac{N^{3 \delta}}{\alpha \pi}} \int_{N^{-\delta}}^{N^{1-\delta}} \sqrt{x} e^{N^{\delta} h(x)} d x\left(1+O\left(N^{1-2 \delta}\right)\right)+R
\end{aligned}
$$

onde

$$
h(x)=x \log \left(\left(\frac{\alpha e}{2 x}\right)^{\frac{2}{\alpha}}(Z \bar{W})\right)=x \log \left(\left(\frac{\alpha e}{2}\right)^{\frac{2}{\alpha}}(Z \bar{W})\right)-\frac{2 x}{\alpha} \log x .
$$


Agora usaremos o método do ponto de sela para estimar a integral que ocorre na expressão (3.69). Este método faz uso do Teorema de Cauchy para deformar o intervalo de integração $\left[N^{-\delta}, N^{1-\delta}\right]$ de (3.69) em uma integral sobre uma curva $\beta$, de forma que $S$ pode ser escrito como

$$
S=\sqrt{\frac{N^{3 \delta}}{\alpha \pi}} \int_{\beta} \sqrt{\eta} e^{N^{\delta} h(\eta)} d \eta\left(1+O\left(N^{1-2 \delta}\right)\right)+R
$$

onde $h: \mathbb{C} \rightarrow \mathbb{C}$ é a extensão analítica de $h$ (dada por (3.70) trocando $x \in \mathbb{R}$ por $\eta \in \mathbb{C}$ ), $d \eta$ é a medida comprimento de arco em $\beta$, que é uma curva suave que liga os pontos

$$
\eta_{1}=\left(N^{-\delta}, 0\right) \text { e } \eta_{2}=\left(N^{1-\delta}, 0\right), \eta_{1}, \eta_{2} \in \mathbb{R}^{2} \simeq \mathbb{C}
$$

pela região de analiticidade das funções no integrando de (3.71) escolhida de forma que esta curva passe pelo ponto de sela, definido como a solução da equação

$$
h^{\prime}\left(\eta_{0}\right)=0
$$

que maximiza a função $\operatorname{Reh}(\eta)$ (caso este ponto não seja único), na direção dada pela curva de nível

$$
\operatorname{Im} h(\eta)=\text { constante }
$$

sempre que

$$
\operatorname{Re} h(\eta) \geq \max \left\{\operatorname{Reh}\left(\eta_{1}\right), \operatorname{Re} h\left(\eta_{2}\right)\right\}
$$

Se a condição acima for satisfeita, então a contribuição principal para a integral (3.71) será dada no ponto de sela $\eta_{0}$; se nunca for satisfeita, então, em particular

$$
\operatorname{Reh}\left(\eta_{0}\right)<\max \left\{\operatorname{Reh}\left(\eta_{1}\right), \operatorname{Re} h\left(\eta_{2}\right)\right\}
$$

e, portanto, a principal contribuição para a integral (3.71) será nos pontos extremos.

Analisemos primeiramente os pontos extremos $\eta_{1}, \eta_{2}$. Assim

$$
h\left(\eta_{1}\right)=N^{-\delta} \log \left(\left(\frac{\alpha e}{2 N^{-\delta}}\right)^{\frac{2}{\alpha}}(Z \bar{W})\right)=O\left(\frac{1}{N^{\delta}}\right) \text { e } \sqrt{\eta_{1}}=O\left(\frac{1}{N^{\frac{\delta}{2}}}\right)
$$

e

$$
h\left(\eta_{2}\right)=N^{1-\delta} \log \left(\left(\frac{\alpha e}{2 N^{1-\delta}}\right)^{\frac{2}{\alpha}}(Z \bar{W})\right)=-\frac{\alpha}{2}(1-\delta) N^{\delta} \log N\left(1+O\left(\frac{1}{\log N}\right)\right) .
$$

Desta forma, nem $\eta_{1}$ nem $\eta_{2}$ terão papel relevante, pois ambos deixam a integral (3.71) no máximo $O(1)$ em $N$ e, portanto, $K_{N}^{\alpha}$ fica exponencialmente pequeno com $N$ (por (3.27) 
e (3.29)). Notemos que toda a análise anterior se baseia na escolha de $\delta \in] \frac{1}{2}, 1[$. Assim, podemos representar (3.71) apenas pela contribuição dada pelo ponto de sela.

Expandimos $h$ em série de Taylor na vizinhança de $\eta_{0}$ temos

$$
\begin{aligned}
h(\eta) & =h\left(\eta_{0}\right)+\frac{1}{2}\left(\eta-\eta_{0}\right)^{2} h^{\prime \prime}\left(\eta_{0}\right)+O\left(\left(\eta-\eta_{0}\right)^{3}\right) \\
& =h\left(\eta_{0}\right)+\frac{1}{2} a r^{2} e^{i(2 \theta+\varphi)}+O\left(\left(\eta-\eta_{0}\right)^{3}\right)
\end{aligned}
$$

onde escrevemos

$$
\eta-\eta_{0}=r e^{i \theta} \in \beta \text { e } h^{\prime \prime}\left(\eta_{0}\right)=a e^{i \varphi} .
$$

Assim, para a escolha de $\beta$ descrito acima, obtemos o seguinte conjunto de equações para $\theta$

$$
2 \theta+\varphi=k \pi \operatorname{e} \operatorname{Reh}(\eta)<\operatorname{Reh}\left(\eta_{0}\right) \operatorname{com} \eta=\eta_{0}+r e^{i \theta} \in \beta
$$

Logo, com esta escolha de $\theta$ e de acordo com o método do ponto de sela (veja detalhes em [34]), podemos aproximar a integral (3.71) por uma integral gaussiana na vizinhança de $\eta_{0}$ na direção dada por (3.74), resultando

$$
S=\sqrt{\frac{N^{3 \delta}}{\alpha \pi}} \sqrt{\eta_{0}} \sqrt{\frac{-2 \pi}{N h^{\prime \prime}\left(\eta_{0}\right)}} e^{N^{\delta} h\left(\eta_{0}\right)}\left(1+O\left(N^{1-2 \delta}\right)\right)+R
$$

onde

$$
\eta_{0}=\frac{\alpha}{2}(Z \bar{W})^{\frac{\alpha}{2}}
$$

é o ponto de sela. De fato, $h^{\prime}\left(\eta_{0}\right)=0$ e, portanto, de (3.70)

$$
\begin{aligned}
h^{\prime}\left(\eta_{0}\right) & =0=\log \left(\left(\frac{\alpha e}{2}\right)^{\frac{2}{\alpha}}(Z \bar{W})\right)-\frac{2}{\alpha} \log \eta_{0}-\frac{2}{\alpha} \\
& =\log \left(\left(\frac{\alpha e}{2}\right)^{\frac{2}{\alpha}} \frac{(Z \bar{W})}{\left(\eta_{0} e\right)^{\frac{2}{\alpha}}}\right) \Rightarrow \\
1 & =\left(\frac{\alpha}{2}\right)^{\frac{2}{\alpha}} \frac{(Z \bar{W})}{\left(\eta_{0}\right)^{\frac{2}{\alpha}}} \Rightarrow \eta_{0}=\frac{\alpha}{2}(Z \bar{W})^{\frac{\alpha}{2}} .
\end{aligned}
$$

Assim, para simplificarmos a expressão (3.75) calculemos

$$
h\left(\eta_{0}\right)=\frac{\alpha}{2}(Z \bar{W})^{\frac{\alpha}{2}} \log \left(\left(\frac{\alpha e}{2 \frac{\alpha}{2}(Z \bar{W})^{\frac{\alpha}{2}}}\right)^{\frac{2}{\alpha}}(Z \bar{W})\right)=\frac{\alpha}{2}(Z \bar{W})^{\frac{\alpha}{2}} \log \left(e^{\frac{2}{\alpha}}\right)=(Z \bar{W})^{\frac{\alpha}{2}}
$$


e

$$
h^{\prime \prime}\left(\eta_{0}\right)=-\frac{2}{\alpha \eta_{0}}=-\frac{2}{\alpha \frac{\alpha}{2}(Z \bar{W})^{\frac{\alpha}{2}}}=-\frac{4}{\alpha^{2}(Z \bar{W})^{\frac{\alpha}{2}}} .
$$

Logo

$$
\begin{aligned}
S & =\sqrt{\frac{N^{3 \delta}}{\alpha \pi}} \sqrt{\eta_{0}} \sqrt{\frac{-2 \pi}{N h^{\prime \prime}\left(\eta_{0}\right)}} e^{N^{\delta} h\left(\eta_{0}\right)}\left(1+O\left(N^{1-2 \delta}\right)\right)+R \\
& =\sqrt{\frac{N^{3 \delta}}{\alpha \pi} \frac{\alpha}{2}(Z \bar{W})^{\frac{\alpha}{2}} \frac{2 \pi \alpha^{2}(Z \bar{W})^{\frac{\alpha}{2}}}{4 N^{\delta}}} e^{N^{\delta}(Z \bar{W})^{\frac{\alpha}{2}}}\left(1+O\left(N^{1-2 \delta}\right)\right)+R \\
& =\frac{\alpha}{2}(Z \bar{W})^{\frac{\alpha}{2}} e^{N^{\delta}(Z \bar{W})^{\frac{\alpha}{2}}} N^{\delta}\left(1+R_{S}\right)
\end{aligned}
$$

onde na última passagem usamos (3.67) com

$$
\begin{aligned}
R_{S} & =O\left(N^{1-2 \delta}\right)+O\left(\frac{\log N}{N^{\frac{\delta}{2}}}\right)+O\left(\frac{(\log N)^{3}}{N}\right) \\
& =O\left(N^{1-2 \delta}\right)+O\left(\frac{\log N}{N^{\frac{\delta}{2}}}\right) .
\end{aligned}
$$

Portanto, obtemos de (3.27)

$$
\begin{aligned}
K_{N}^{\alpha}\left(\frac{Z}{N^{\gamma}}, \frac{W}{N^{\gamma}}\right) & =\frac{\alpha e^{-\frac{N}{2}\left|\frac{Z}{N^{\gamma}}\right|^{\alpha}} e^{-\frac{N}{2}\left|\frac{W}{N^{\gamma}}\right|^{\alpha}} N^{2 \gamma+\delta}}{2 \pi Z \bar{W}} \frac{\alpha(Z \bar{W})^{\frac{\alpha}{2}}}{2} e^{N^{\delta}(Z \bar{W})^{\frac{\alpha}{2}}}\left(1+R_{S}\right) \\
& =\frac{\alpha^{2}}{4 \pi} N^{\delta+2 \gamma}(Z \bar{W})^{\frac{\alpha}{2}-1} e^{N^{\delta}\left((Z \bar{W})^{\frac{\alpha}{2}-\frac{|Z|^{\alpha}}{2}-\frac{|W|^{\alpha}}{2}}\right)}\left(1+R_{S}\right)
\end{aligned}
$$

onde na última passagem foi usado que

$$
\alpha \gamma+\delta=1 \operatorname{com} \frac{1}{2}<\delta<1
$$

Logo,

$$
\frac{1}{N^{\delta+2 \gamma}} K_{N}^{\alpha}\left(\frac{Z}{N^{\gamma}}, \frac{W}{N^{\gamma}}\right)=\frac{\alpha^{2}}{4 \pi}(Z \bar{W})^{\frac{\alpha}{2}-1} e^{N^{\delta}\left((Z \bar{W})^{\frac{\alpha}{2}-\frac{|Z|^{\alpha}}{2}-\frac{|W|^{\alpha}}{2}}\right)}\left(1+R_{S}\right)
$$

como queríamos. Em particular, tomando $\delta / 1$ respeitando as equações (3.76) e (3.77) (conseqüentemente $\gamma \searrow 0$ ) obtemos

$$
\frac{1}{N} K_{N}^{\alpha}(Z, W)=\frac{\alpha^{2}}{4 \pi}(Z \bar{W})^{\frac{\alpha}{2}-1} e^{N\left((Z \bar{W})^{\frac{\alpha}{2}}-\frac{|Z|^{\alpha}}{2}-\frac{|W|^{\alpha}}{2}\right)}\left(1+O\left(\frac{\log N}{\sqrt{N}}\right)\right) .
$$


Observação 77 A introdução das escalas $\gamma$ e $\delta$ na prova do teorema anterior são fundamentais para utilizarmos o método do ponto de sela. Com o uso destas escalas, além de garantirmos que a principal contribuição venha do ponto de sela, conseguimos estimar as ordens dos erros que estamos cometendo usando esta expressão assintótica em relação à expressão exata. Notemos que a densidade $\rho^{V}$ associada à medida de equilíbrio $\mu^{V_{\alpha}}\left(V_{\alpha}\right.$ dado por 3.9) é dada por

$$
\rho^{V}(z)=\lim _{N \rightarrow \infty} \frac{1}{N} K_{N}(z, z)=\frac{\alpha^{2}}{4 \pi}|z|^{\alpha-2}
$$

por (3.79) que está de acordo com o Corolário D.1 do Apêndice. Notemos também que as equações (3.79) para $\alpha=2$ e (E.9) para $\tau=0$ coincidem, pois ambas representam o núcleo integral do ensemble de Ginibre. 



\section{Capítulo 4}

\section{Universalidade para o ensemble unitário normal}

Neste Capítulo discutiremos a questão da universalidade como no texto de Deift [7] (veja na Seção seguinte).

\subsection{Universalidade para o ensemble unitário hermi- tiano}

Consideramos o ensemble unitário hermitiano de matrizes hermitianas $H_{N}$ distribuídas de acordo com

$$
P_{N}^{V_{H}}\left(H_{N}\right) d H_{N}=\widetilde{Z}_{N}^{-1} e^{-N T r V_{H}\left(H_{N}\right)} d H_{N}
$$

onde $\widetilde{Z}_{N}^{-1}$ é um fator de normalização e $V_{H}: \mathbb{R} \longrightarrow \mathbb{R}$ uma função contínua real-analítica satisfazendo

$$
\lim _{|\lambda| \rightarrow \infty} \frac{V_{H}(\lambda)}{\log \left(1+\lambda^{2}\right)}=\infty
$$

(notamos que esta hipótese é similar à hipótese iii. do Teorema 40). Em termos dos elementos espectrais, a medida de probabilidade dos autovalores $\left\{\lambda_{i}\right\}_{i=1}^{N}$ de $H_{N}$ pode ser escrita como

$$
\begin{aligned}
P_{N}^{V_{H}}\left(\lambda_{1}, \cdots, \lambda_{N}\right) & =Z_{N}^{-1} \prod_{1 \leq i<j \leq N}\left|\lambda_{i}-\lambda_{j}\right|^{2} e^{-N \sum_{i=1}^{N} V_{H}\left(\lambda_{i}\right)} \\
& =Z_{N}^{-1} e^{-\left(\sum_{1 \leq i \neq j \leq N} \log \left|\lambda_{i}-\lambda_{j}\right|^{-1}+N \sum_{i=1}^{N} V_{H}\left(\lambda_{i}\right)\right)} \\
& =Z_{N}^{-1} e^{-N^{2} I^{V}\left(\mu_{N}\right)}
\end{aligned}
$$


onde

$$
d \mu_{N}(\lambda)=N^{-1} \sum_{i=1}^{N} \delta\left(\lambda-\lambda_{i}\right) d \lambda
$$

é a medida empírica dos autovalores de $M_{N}$,

$$
I^{V}(\mu) \equiv \int\left(V(\lambda)+U^{\mu}(\lambda)\right) d \mu(\lambda)
$$

é a "energia eletrostática" associada à $\mu$ (veja Observação a seguir) e

$$
U^{\mu}(\lambda)=\int \log |\lambda-x|^{-1} d \mu(x)
$$

é o potencial logarítmico associado a $\mu$.

Observação 78 Notemos que a teoria discutida no Capítulo 2 se aplica aos ensembles tratados nesta Seção. Por exemplo, se tomarmos

$$
E^{V} \equiv \inf _{\mu \in \mathcal{M}(\mathbb{R})} I^{V}(\mu)=I\left(\mu^{V}\right), \quad E^{V}<\infty
$$

onde $\mathcal{M}(\mathbb{R})$ é o conjunto de todas as medidas de probabilidade em $\mathbb{R}$, então $\mu^{V_{H}}$ é a medida de equilíbrio associada ao ensemble unitário hermitiano.

Para investigar a universalidade da estatística local dos autovalores, consideramos a probabilidade $E_{N}^{V_{H}}(a, b)$ de não haver autovalores num intervalo $(a, b) \subset \mathbb{R}$, ou seja

$$
E_{N}^{V_{H}}(a, b)=\int P_{N}^{V_{H}}\left(\lambda_{1}, \cdots, \lambda_{N}\right) \prod_{i=1}^{N} \chi_{\mathbb{R} \backslash(a, b)}\left(\lambda_{i}\right) d \lambda_{i}
$$

onde $\chi_{\mathbb{A}}$ é a função indicadora do conjunto $\mathbb{A}$. A expressão

$$
E_{N}^{V_{H}}(a, b)=\operatorname{det}\left(I-K_{N}^{U}\right)
$$

pode ser escrita como um determinante de Fredholm, com $I$ o operador identidade e $K_{N}^{U}$ o operador integral em $L^{2}((a, b) ; \mathbb{R})$ com núcleo integral

$$
K_{N}^{U}(x, y)=e^{-\frac{N}{2}\left(V_{H}(x)+V_{H}(y)\right)} \sum_{j=1}^{N} \phi_{j}(x) \phi_{j}(y)
$$

onde $\left\{\phi_{j}\right\}_{j=1}^{N}$ denota o conjunto dos polinômios ortonormais até ordem $N-1$ com respeito ao peso

$$
d \nu(x)=e^{-N V_{H}(x)} d x
$$


Notamos que para $(a, b) \subset \mathbb{S}^{V_{H}} \subset \mathbb{R}$ teremos

$$
\lim _{N \rightarrow \infty} E_{N}^{V_{H}}(a, b)=0
$$

pois, quando $N \rightarrow \infty$, os autovalores se adensam no suporte $\mathbb{S}^{V_{H}}$ da medida de equilíbrio $\mu^{V_{H}}$ e, portanto, não há intervalos contidos em $\mathbb{S}^{V_{H}}$ sem autovalores no limite $N \rightarrow \infty$. Portanto, precisamos encontrar uma escala para examinar o comportamento assintótico de $E_{N}^{V_{H}}$ para $N$ grande. Usualmente, adota-se a seguinte escala usada em [20] é

$$
E_{N}^{V_{H}}\left(\lambda, \lambda+\frac{s}{K_{N}^{U}(\lambda, \lambda)}\right)=\operatorname{det}\left(I-\widetilde{K}_{N}^{U}\right)
$$

onde $\widetilde{K}_{N}^{U}$ é um o operador integral em $L^{2}((0, s) ; \mathbb{R})$ dado por

$$
\widetilde{K}_{N}^{U}(x, y)=\frac{1}{K_{N}^{U}(\lambda, \lambda)} K_{N}^{U}\left(\lambda+\frac{x}{K_{N}^{U}(\lambda, \lambda)}, \lambda+\frac{y}{K_{N}^{U}(\lambda, \lambda)}\right)
$$

(veja [20]).

Observação 79 Analogamente ao que ocorre na Observação 71 temos que

$$
K_{N}^{U}(\lambda, \lambda)=N \rho^{V_{H}}(\lambda)(1+o(1))
$$

onde $\rho^{V_{H}}$ é a densidade de probabilidade associada à medida de equilíbrio $\mu^{V_{H}}$.

A escala é escolhida de forma que o valor esperado do número de autovalores no intervalo de comprimento s próximo a um ponto $\lambda$ pertencente ao suporte da medida de equilíbrio $\mu^{V_{H}}$ seja $s$ (espaçamento médio unitário). De fato, se

$$
f_{N}(\mathbb{A})=N^{-1} \sum_{i=1}^{N} \chi_{\mathbb{A}}\left(\lambda_{i}\right)
$$

é a fração dos autovalores de $H_{N}$ no intervalo $\mathbb{A} \subset \mathbb{R}$ então, para $N$ grande,

$$
\mathbb{E}\left(N f_{N}\left(\left[\lambda, \lambda+\frac{s}{K_{N}^{U}(\lambda, \lambda)}\right]\right)\right)=N \int_{\lambda}^{\lambda+\frac{s}{N \rho V^{H}(\lambda)}} \rho^{V_{H}}\left(\lambda^{\prime}\right) d \lambda^{\prime}(1+o(1))=s(1+o(1))
$$

onde a esperança $\mathbb{E}$ é tomada com respeito à (4.1).

Deift et al [20] mostram que o limite

$$
\lim _{N \rightarrow \infty} \widetilde{K}_{N}^{U}(x, y)=S(x, y)
$$

existe pontualmente, independentemente de $V_{H}$ e

$$
\lim _{N \rightarrow \infty} E_{N}^{V_{H}}\left(a, a+\frac{s}{K_{N}^{U}(\lambda, \lambda)}\right)=\operatorname{det}(I-S)
$$


onde $S$ é o operador integral em $L^{2}((0, s) ; \mathbb{R})$ com núcleo integral

$$
S(x, y)=\frac{\operatorname{sen} \pi(x-y)}{\pi(x-y)}
$$

Pelo fato de $S$ não depender de $V_{H}$, os ensembles unitários hermitianos são, neste sentido, universais.

\subsection{Escala para o ensemble unitário normal}

Vamos agora adaptar as questões formuladas na Seção anterior para a família de ensembles unitários normais com potencial radialmente simétrico do tipo

$$
V_{\alpha}(z)=|z|^{\alpha}
$$

A distribuição dos autovalores relativa a este potencial é

$$
P_{N}^{V_{\alpha}}\left(z_{1}, \cdots, z_{N}\right)=Z_{N}^{-1} \prod_{1 \leq i<j \leq N}\left|z_{i}-z_{j}\right|^{2} e^{-N \sum_{i=1}^{N} V_{\alpha}\left(z_{i}\right)}
$$

e devemos obter para este ensemble uma escala de tal forma que o espaçamento médio entre os autovalores na vizinhança da origem seja unitário (veja equação (4.4)).

Prova do Teorema 28. Seguindo as indicações sugeridas pela expressão (4.4) temos que encontrar uma função $g_{\alpha}(s)$ que desempenha o papel de

$$
g_{H}(s)=\frac{s}{N \rho^{V_{H}}(0)}
$$

na vizinhança de $\lambda=0$ no ensemble unitário hermitiano, de tal forma que

$$
\mathbb{E}\left(N f_{N}\left(\mathbb{D}_{s}\right)\right)=s(1+o(1))
$$

onde $\mathbb{D}_{s}$ é um disco de raio $s$ centrado na origem. A função $g_{\alpha}$ pode depender de $s, N$ e de $\alpha$.

Explicitamente através da densidade de equilíbrio $\rho^{\alpha}(r)$, fazendo $z=r e^{i \theta}$ na equação 
análoga a (4.4) temos

$$
\begin{aligned}
\mathbb{E}\left(N f_{N}\left(\mathbb{D}_{g_{\alpha}(s)}\right)\right) & =N \int_{0}^{2 \pi} \int_{0}^{g_{\alpha}(s)} \rho^{\alpha}(r) r d r d \theta(1+o(1)) \\
& =N \int_{0}^{2 \pi} \int_{0}^{g_{\alpha}(s)} \frac{\alpha^{2}}{4 \pi} r^{\alpha-2} r d r d \theta(1+o(1)) \\
& =\left.\frac{N \alpha^{2} 2 \pi}{4 \pi} \frac{r^{\alpha}}{\alpha}\right|_{0} ^{g_{\alpha}(s)}(1+o(1)) \\
& =\frac{N \alpha}{2}\left(\left(g_{\alpha}(s)\right)^{\alpha}\right)(1+o(1)) \\
& \equiv \pi s^{2}(1+o(1))
\end{aligned}
$$

e, portanto,

$$
\frac{N \alpha}{2}\left(g_{\alpha}(s)\right)^{\alpha}=\pi s^{2} \Longrightarrow g_{\alpha}(s)=\left(\frac{2 \pi s^{2}}{N \alpha}\right)^{\frac{1}{\alpha}}
$$

como queríamos.

Observação 80 Notamos que não podemos realizar o procedimento implícito adotado em (4.4) para determinarmos esta escala, pois exceto para $\alpha=2$, a densidade de equilíbrio $\rho^{\alpha}(r)$ ou a sua recíproca $\frac{1}{\rho^{\alpha}(r)}$ é não limitada em $r=0$. Para $\alpha=2$ temos

$$
\rho^{\alpha}(r)=\left\{\begin{array}{l}
\frac{1}{\pi} \text { se } r<1 \\
0 \text { se } r>1
\end{array}\right.
$$

e, portanto,

$$
\begin{aligned}
\mathbb{E}\left(N f_{N}\left(\mathbb{D}_{g_{2}(s)}\right)\right) & =N \int_{\mathbb{D}_{g_{2}(s)}} \frac{1}{\pi} d^{2} z(1+o(1)) \\
& =\frac{N}{\pi} \pi\left(\left(\frac{2 \pi s^{2}}{N 2}\right)^{\frac{1}{2}}\right)^{2}(1+o(1))=\pi s^{2}(1+o(1))
\end{aligned}
$$

o que mostra a concordância entre este cálculo implícito e o usado em (4.9).

Observação 81 A escala (4.10) é dependente do potencial $V_{\alpha}$, o que não ocorre no ensemble unitário hermitiano (4.8). 


\subsection{Limite do núcleo integral escalado}

Mostramos na Seção 3.1.1 que o núcleo integral $K_{N}^{\alpha}$ associado à $V_{\alpha}$ dado 4.6 é

$$
\frac{1}{N} K_{N}^{\alpha}(Z, W)=\frac{\alpha^{2}}{4 \pi}(Z \bar{W})^{\frac{\alpha}{2}-1} e^{N\left((Z \bar{W})^{\frac{\alpha}{2}-\frac{|Z|^{\alpha}}{2}-\frac{|W|^{\alpha}}{2}}\right)}\left(1+O\left(\frac{\log N}{\sqrt{N}}\right)\right) .
$$

Partindo desta expressão podemos concluir a

Prova do Teorema 29. Considerando apenas termos de ordem superior em $N$ temos

$$
\begin{aligned}
& \widetilde{K}_{N}^{\alpha}(z, w)=g_{\alpha}^{\prime}(z) K_{N}^{\alpha}\left(g_{\alpha}(z), g_{\alpha}(w)\right) \overline{g_{\alpha}^{\prime}(w)} \\
& =\frac{\alpha^{2} N}{4 \pi}\left(\frac{4 \pi}{N \alpha^{2}}\right)^{2} z \bar{w}\left(\frac{2 \pi z \bar{w}}{N \alpha}\right)^{\frac{2}{\alpha}-2}\left(\left(\frac{2 \pi z^{2}}{N \alpha}\right)^{\frac{1}{\alpha}} \overline{\left(\frac{2 \pi w^{2}}{N \alpha}\right)^{\frac{1}{\alpha}}}\right)^{\frac{\alpha}{2}-1} \\
& \times e^{N\left(\left(\left(\frac{2 \pi z^{2}}{N \alpha}\right)^{\frac{1}{\alpha}} \overline{\left(\frac{2 \pi w^{2}}{N \alpha}\right)^{\frac{1}{\alpha}}}\right)^{\frac{\alpha}{2}}-\frac{\left|\left(\frac{2 \pi z^{2}}{N \alpha}\right)^{\frac{1}{\alpha}}\right|^{\alpha}}{2}-\frac{\left|\left(\frac{2 w}{N \alpha}\right)^{\frac{1}{\alpha}}\right|^{\alpha}}{2}\right)} \\
& =\frac{\alpha^{2-4-\frac{2}{\alpha}+2-\frac{2}{\alpha}\left(\frac{\alpha}{2}-1\right)} N^{1-2-\frac{2}{\alpha}+2-\frac{2}{\alpha}\left(\frac{\alpha}{2}-1\right)}}{2^{2-4-\frac{2}{\alpha}+2-\frac{2}{\alpha}\left(\frac{\alpha}{2}-1\right)} \pi^{1-2-\frac{2}{\alpha}+2-\frac{2}{\alpha}\left(\frac{\alpha}{2}-1\right)}}(z \bar{w})^{1+\frac{2}{\alpha}-2+\frac{2}{\alpha}\left(\frac{\alpha}{2}-1\right)} \\
& \times e^{N}\left(\left(\left(\frac{2 \pi z^{2}}{N \alpha}\right) \overline{\left(\frac{2 \pi w^{2}}{N \alpha}\right)}\right)^{\frac{1}{2}}-\frac{\left(\frac{2\left|\pi z^{2}\right|}{N \alpha}\right)}{2}-\frac{\left(\frac{2\left|\pi w^{2}\right|}{N \alpha}\right)}{2}\right) \\
& =\frac{2}{\alpha} e^{\frac{2 \pi}{\alpha}\left(z \bar{w}-\frac{|z|^{2}}{2}-\frac{|w|^{2}}{2}\right)} .
\end{aligned}
$$

Pela definição de $S^{\alpha}(z, w)$ em (1.41) temos que

$$
\lim _{N \rightarrow \infty} \widetilde{K}_{N}^{\alpha}(z, w)=S^{\alpha}(z, w)
$$

como queríamos.

\subsubsection{Probabilidade de um disco aberto na vizinhança da origem não conter autovalores}

Nesta seção vamos calcular a probabilidade $E_{N}^{V_{\alpha}}(r)$ de não haver autovalores em

$$
\mathbb{B}_{0, r}=\{z \in \mathbb{C}:|z|<r\},
$$


uma bola aberta centrada na origem de raio $r$ :

$$
E_{N}^{V_{\alpha}}(r)=\int P_{N}^{V_{\alpha}}\left(z_{1}, \cdots, z_{N}\right) \prod_{k=1}^{N} \chi_{\mathbb{R} \backslash \mathbb{B}_{0, r}}\left(z_{k}\right) d^{2} z_{k}
$$

onde

$$
P_{N}^{V_{\alpha}}\left(z_{1}, \cdots, z_{N}\right)=Z_{N}^{-1} \prod_{1 \leq i<j \leq N}\left|z_{i}-z_{j}\right|^{2} e^{-N \sum_{k=1}^{N} V_{\alpha}\left(z_{k}\right)}
$$

com $V_{\alpha}\left(z_{k}\right)=\left|z_{k}\right|^{\alpha}$ e $\chi_{\mathbb{A}}$ é a função indicadora do conjunto $\mathbb{A} \mathrm{e}$

$$
Z_{N}=\int \prod_{1 \leq i<j \leq N}\left|z_{i}-z_{j}\right|^{2} e^{-N \sum_{k=1}^{N} V_{\alpha}\left(z_{k}\right)} \prod_{k=1}^{N} d^{2} z_{k}
$$

é a normalização de $P_{N}^{V_{\alpha}}$.

Prova do Teorema 30. Podemos escrever $\prod_{1 \leq j<i \leq N}\left(z_{i}-z_{j}\right)$ como o determinante de van der Monde como

$$
\prod_{1 \leq j<i \leq N}\left(z_{i}-z_{j}\right)=\operatorname{det}\left(\begin{array}{ccc}
1 & \cdots & 1 \\
z_{1} & \cdots & z_{N} \\
\vdots & \ddots & \vdots \\
z_{1}^{N-1} & \cdots & z_{N}^{N-1}
\end{array}\right)
$$


Portanto,

$$
\begin{aligned}
& \prod_{1 \leq i<j \leq N}\left|z_{i}-z_{j}\right|^{2} \\
& =\operatorname{det}\left(\begin{array}{ccc}
1 & \ldots & 1 \\
\bar{z}_{1} & \ldots & \bar{z}_{N} \\
\vdots & \ddots & \vdots \\
\bar{z}_{1}^{N-1} & \ldots & \bar{z}_{N}^{N-1}
\end{array}\right) \operatorname{det}\left(\begin{array}{ccc}
1 & \cdots & 1 \\
z_{1} & \cdots & z_{N} \\
\vdots & \ddots & \vdots \\
z_{1}^{N-1} & \cdots & z_{N}^{N-1}
\end{array}\right) \\
& =\operatorname{det}\left(\begin{array}{ccc}
1 & \cdots & 1 \\
\bar{z}_{1} & \cdots & \bar{z}_{N} \\
\vdots & \ddots & \vdots \\
\bar{z}_{1}^{N-1} & \cdots & \bar{z}_{N}^{N-1}
\end{array}\right) \operatorname{det}\left(\begin{array}{cccc}
1 & z_{1} & \cdots & z_{1}^{N-1} \\
\vdots & \vdots & \ddots & \vdots \\
1 & z_{N} & \cdots & z_{N}^{N-1}
\end{array}\right) \\
& =\operatorname{det}\left(\begin{array}{cccc}
\sum_{k=1}^{N} 1 & \sum_{k=1}^{N} z_{k} & \cdots & \sum_{k=1}^{N} z_{k}^{N-1} \\
\sum_{k=1}^{N} \bar{z}_{k} & \sum_{k=1}^{N} \bar{z}_{k} z_{k} & \cdots & \sum_{k=1}^{N} \bar{z}_{k} z_{k}^{N-1} \\
\vdots & \vdots & \ddots & \vdots \\
\sum_{k=1}^{N} \bar{z}_{1}^{N-1} & \sum_{k=1}^{N} \bar{z}_{k}^{N-1} z_{k} & \cdots & \sum_{k=1}^{N}\left(z_{k} \bar{z}_{k}\right)^{N-1}
\end{array}\right) .
\end{aligned}
$$

Desenvolvendo a primeira linha deste determinante pelo método de Laplace, temos

$$
\prod_{1 \leq i<j \leq N}\left|z_{i}-z_{j}\right|^{2}=\sum_{l=1}^{N} \sum_{k=1}^{N} z_{k}^{l-1} C_{1 l}
$$

onde $\left\{C_{1 l}\right\}_{l=1}^{N}$ são os cofatores da primeira linha de (4.14). De (4.12) e (4.13), notamos que $E_{N}^{V_{\alpha}}(r)$ é invariante por permutação das variáveis de integração $z_{i}$. Assim, para o cálculo de $E_{N}^{V_{\alpha}}(r)$ podemos trocar (4.15) por

$$
N \sum_{l=1}^{N} z_{1}^{l-1} C_{1 l}=N \operatorname{det}\left(\begin{array}{ccccc}
1 & z_{1} & \cdots & z_{1}^{N-1} \\
\sum_{k=1}^{N} \bar{z}_{k} & \sum_{k=1}^{N} \bar{z}_{k} z_{k} & \cdots & \sum_{k=1}^{N} \bar{z}_{k} z_{k}^{N-1} \\
\vdots & \vdots & \ddots & \vdots \\
\sum_{k=1}^{N} \bar{z}_{1}^{N-1} & \sum_{k=1}^{N} \bar{z}_{k}^{N-1} z_{k} & \cdots & \sum_{k=1}^{N}\left(z_{k} \bar{z}_{k}\right)^{N-1}
\end{array}\right)
$$


Agora, multiplicando a primeira linha por $-\bar{z}_{1}$ e somando com a segunda linha obtemos

$$
N \sum_{l=1}^{N} z_{1}^{l-1} C_{1 l}=N \operatorname{det}\left(\begin{array}{ccccc}
1 & z_{1} & \cdots & z_{1}^{N-1} \\
\sum_{k=2}^{N} \bar{z}_{k} & \sum_{k=2}^{N} \bar{z}_{k} z_{k} & \cdots & \sum_{k=2}^{N} \bar{z}_{k} z_{k}^{N-1} \\
\vdots & \vdots & \ddots & \vdots \\
\sum_{k=1}^{N} \bar{z}_{1}^{N-1} & \sum_{k=1}^{N} \bar{z}_{k}^{N-1} z_{k} & \cdots & \sum_{k=1}^{N}\left(z_{k} \bar{z}_{k}\right)^{N-1}
\end{array}\right)
$$

e, novamente pela simetria, para todo $z_{i}$ de $E_{N}^{V_{\alpha}}(r)$ podemos substituir a expressão acima para o cálculo de $E_{N}^{V_{\alpha}}(r)$ por

$$
N(N-1) \operatorname{det}\left(\begin{array}{cccc}
1 & z_{1} & \cdots & z_{1}^{N-1} \\
\bar{z}_{2} & \bar{z}_{2} z_{2} & \cdots & \bar{z}_{2} z_{2}^{N-1} \\
\sum_{k=1}^{N} \bar{z}_{k}^{2} & \sum_{k=1}^{N} \bar{z}_{k}^{2} z_{k} & \cdots & \sum_{k=2}^{N} \bar{z}_{k}^{2} z_{k}^{N-1} \\
\vdots & \vdots & \ddots & \vdots \\
\sum_{k=1}^{N} \bar{z}_{1}^{N-1} & \sum_{k=1}^{N} \bar{z}_{k}^{N-1} z_{k} & \cdots & \sum_{k=1}^{N}\left(z_{k} \bar{z}_{k}\right)^{N-1}
\end{array}\right) .
$$

Seguindo este raciocínio, podemos escrever $\prod_{1 \leq i<j \leq N}\left|z_{i}-z_{j}\right|^{2}$ para o cálculo de $E_{N}^{V_{\alpha}}(r)$ como

$$
N ! \operatorname{det}\left(\begin{array}{cccc}
1 & z_{1} & \cdots & z_{1}^{N-1} \\
\bar{z}_{2} & \bar{z}_{2} z_{2} & \cdots & \bar{z}_{2} z_{2}^{N-1} \\
\vdots & \vdots & \ddots & \vdots \\
\bar{z}_{1}^{N-1} & \bar{z}_{N}^{N-1} z_{N} & \cdots & \left(z_{N} \bar{z}_{N}\right)^{N-1}
\end{array}\right)
$$

Como $V_{\alpha}(z)=|z|^{\alpha}$ e, portanto, possui simetria radial, somente os termos diagonais vão contribuir para o cálculo de $E_{N}^{V_{\alpha}}(r)$, pois para todo $k$

$$
\int_{\left|z_{k}\right| \geq s} \bar{z}_{k}^{l} z_{k}^{m} e^{-N V_{\alpha}\left(z_{k}\right)} d^{2} z_{k}=2 \pi \delta_{l m} \int_{s}^{\infty} r_{k}^{2 l+1} e^{-N r_{k}^{\alpha}} d r_{k}
$$


onde $z_{k}=r_{k} e^{i \theta_{k}}$. Assim,

$$
\begin{aligned}
E_{N}^{V_{\alpha}}(r)= & \int P_{N}^{V_{\alpha}}\left(z_{1}, \cdots, z_{N}\right) \prod_{k=1}^{N} \chi_{\mathbb{R} \backslash \mathbb{B}_{0, r}}\left(z_{k}\right) d^{2} z_{k} \\
= & \frac{N !(2 \pi)^{N} \int_{r}^{\infty} \prod_{k=1}^{N}\left(\bar{z}_{k} z_{k}\right)^{k-1} e^{-N \sum_{k=1}^{N}\left|z_{k}\right|^{\alpha}}\left|z_{k}\right| d\left|z_{k}\right|}{N !(2 \pi)^{N} \int_{0}^{\infty} \prod_{k=1}^{N}\left(\bar{z}_{k} z_{k}\right)^{k-1} e^{-N \sum_{k=1}^{N}\left|z_{k}\right|^{\alpha}}\left|z_{k}\right| d\left|z_{k}\right|} \\
= & \prod_{k=1}^{N} \frac{\int_{r}^{\infty} r_{k}^{2 k-1} e^{-N r_{k}^{\alpha}} d r_{k}}{\int_{k}^{2 k-1} e^{-N r_{k}^{\alpha}} d r_{k}}
\end{aligned}
$$

fazendo a mudança de variável

$$
\begin{aligned}
N r_{k}^{\alpha} & =t_{k} \\
& \Rightarrow d t_{k}=\alpha N r_{k}^{\alpha-1} d r_{k}=\alpha N\left(\frac{t_{k}}{N}\right)^{\frac{\alpha-1}{\alpha}} d r_{k} \\
& \Rightarrow d r_{k}=\frac{1}{\alpha N}\left(\frac{t_{k}}{N}\right)^{\frac{1-\alpha}{\alpha}} d t_{k}
\end{aligned}
$$

obtemos

$$
\begin{aligned}
E_{N}^{V_{\alpha}}(r) & =\prod_{k=1}^{N} \frac{\int_{N r^{\alpha}}^{\infty}\left(\frac{t_{k}}{N}\right)^{\frac{2 k-1}{\alpha}} e^{-t_{k}} \frac{1}{\alpha N}\left(\frac{t_{k}}{N}\right)^{\frac{1-\alpha}{\alpha}} d t_{k}}{\int_{0}^{\infty}\left(\frac{t_{k}}{N}\right)^{\frac{2 k-1}{\alpha}} e^{-t_{k}} \frac{1}{\alpha N}\left(\frac{t_{k}}{N}\right)^{\frac{1-\alpha}{\alpha}} d t_{k}} \\
& =\prod_{k=1}^{N} \frac{\int_{N r^{\alpha}}^{\infty} t_{k}^{\frac{2 k}{\alpha}-1} e^{-t_{k}} d t_{k}}{\int_{0}^{\infty} t_{k}^{\frac{2 k}{\alpha}-1} e^{-t_{k}} d t_{k}} \\
& =\prod_{k=1}^{N} \frac{\Gamma\left(\frac{2 k}{\alpha}, N r^{\alpha}\right)}{\Gamma\left(\frac{2 k}{\alpha}\right)}
\end{aligned}
$$

como queríamos.

Observação 82 Este Teorema pode ser enunciado de maneira mais geral da seguinte forma: Seja

$$
E_{N}^{V_{C}}(r) \equiv E_{N}^{V_{C}}\left(\mathbb{B}_{0, r}\right)=\int P_{N}^{V_{C}}\left(z_{1}, \cdots, z_{N}\right) \prod_{k=1}^{N} \chi_{\mathbb{R} \backslash \mathbb{B}_{0, r}}\left(z_{k}\right) d^{2} z_{k}
$$

e $V_{C}: \mathbb{C} \rightarrow \mathbb{R}$ é um potencial como no Teorema 40 ,

$$
V_{C}(z) \equiv V_{C}(r), \quad z=r e^{i \theta}
$$


(simetria radial), convexo e crescente (hipóteses para garantir a existência de autovalores na vizinhança de $z=0$ ). Então,

$$
E_{N}^{V_{C}}(r)=\prod_{k=1}^{N} \frac{\int_{r}^{\infty} r_{k}^{2 k-1} e^{-N V_{C}\left(r_{k}\right)} d r_{k}}{\int_{0}^{\infty} r_{k}^{2 k-1} e^{-N V_{C}\left(r_{k}\right)} d r_{k}} .
$$

Esta prova segue exatamente os passos da prova do Teorema anterior até a equação (4.16).

Notemos que como

$$
E_{N}^{V_{\alpha}}(r)=\prod_{k=1}^{N} \frac{\Gamma\left(\frac{2 k}{\alpha}, N r^{\alpha}\right)}{\Gamma\left(\frac{2 k}{\alpha}\right)}
$$

Então

$$
\lim _{N \rightarrow \infty} E_{N}^{V_{\alpha}}(r)=0, \forall r>0 .
$$

Em palavras, com probabilidade 1 não encontramos discos abertos na origem com ausência de autovalores, o que está de acordo com o fato da medida de equilíbrio ser absolutamente contínua com suporte compacto contendo a origem nesta escala.

Na próxima seção usaremos a escala (4.10) para encontrarmos uma medida mais apropriada para a ausência de autovalores num aberto centrado na origem.

\subsubsection{Probabilidade escalada de um disco aberto não conter au- tovalores}

Prova do Corolário 31. De (4.17) e (4.10) temos

$$
\begin{aligned}
E_{N}^{V_{\alpha}}\left(g_{\alpha}(s)\right) & =\prod_{k=1}^{N} \frac{\Gamma\left(\frac{2 k}{\alpha}, N\left(\left(\frac{2 \pi s^{2}}{N \alpha}\right)^{\frac{1}{\alpha}}\right)^{\alpha}\right)}{\Gamma\left(\frac{2 k}{\alpha}\right)} \\
& =\prod_{k=1}^{N} \frac{\Gamma\left(\frac{2 k}{\alpha}, \frac{2 \pi s^{2}}{\alpha}\right)}{\Gamma\left(\frac{2 k}{\alpha}\right)}
\end{aligned}
$$

e

como queríamos.

$$
\lim _{N \rightarrow \infty} E_{N}^{V_{\alpha}}\left(g_{\alpha}(s)\right)=\prod_{k=1}^{\infty} \frac{\Gamma\left(\frac{2 k}{\alpha}, \frac{2 \pi s^{2}}{\alpha}\right)}{\Gamma\left(\frac{2 k}{\alpha}\right)}
$$

Observação 83 Notamos que $E_{N}^{V_{\alpha}}\left(g_{\alpha}(s)\right)$, assim como $S^{\alpha}(z, w)$ depende explicitamente de $\alpha$. Já no ensemble unitário hermitiano esta grandeza é dada por

$$
\lim _{N \rightarrow \infty} E_{N}^{V_{H}}\left(a, a+\frac{s}{K_{N}^{U}(\lambda, \lambda)}\right)=\operatorname{det}(I-S)
$$

onde $S$ é dado por (4.5), que não depende do potencial $V_{H}$. 


\subsection{Universalidade Conforme}

Nesta seção mostraremos que o núcleo integral

$$
K_{N}^{\alpha}(z, w)=\frac{\alpha^{2}}{4 \pi} N(z \bar{w})^{\frac{\alpha}{2}-1} e^{N\left((z \bar{w})^{\frac{\alpha}{2}}-\frac{|z|^{\alpha}}{2}-\frac{|w|^{\alpha}}{2}\right)}\left(1+O\left(\frac{\log N}{\sqrt{N}}\right)\right)
$$

associado à família de potenciais do tipo

$$
V_{\alpha}(z)=|z|^{\alpha}
$$

(como no Teorema 27), pode ser obtido pelo núcleo integral universal

$$
K^{U}(z, w)=\frac{1}{\pi} e^{z \bar{w}-\frac{|z|^{2}}{2}-\frac{|w|^{2}}{2}}
$$

a partir da transformação conforme definida por

$$
\varphi_{N}^{\alpha}(z)=\sqrt{N} z^{\frac{\alpha}{2}}
$$

Observação 84 Notemos que $K^{U}$ pode ser entendido como o núcleo integral

$$
\begin{aligned}
K^{U}(z, w) & =e^{-\frac{|z|^{2}}{2}} e^{-\frac{|w|^{2}}{2}} \sum_{j=1}^{\infty} \phi_{j}^{U}(z) \overline{\phi_{j}^{U}(w)} \\
& =e^{-\frac{|z|^{2}}{2}} e^{-\frac{|w|^{2}}{2}} \sum_{j=1}^{\infty} \frac{(z \bar{w})^{j-1}}{\pi \Gamma(j)} \\
& =\frac{1}{\pi} e^{z \bar{w}-\frac{|z|^{2}}{2}-\frac{|w|^{2}}{2}}
\end{aligned}
$$

onde $\left\{\phi_{j}^{U}\right\}_{j=1}^{\infty}$ são os monômios ortonormais com respeito ao peso

$$
d \nu(z)=e^{-|z|^{2}} d^{2} z
$$

Notemos também que $K^{U}$ é um núcleo reprodutor no espaço $\mathcal{H}\left(\mathbb{C}, e^{-|z|^{2}}\right)$ das funções do tipo

$$
f(z) e^{-\frac{|z|^{2}}{2}}
$$

com $f$ analítica em $\mathbb{C}$ e com norma $\|f\|_{\nu}<\infty$. De fato, como o conjunto $\left\{\phi_{j}^{U}\right\}_{j=1}^{\infty}$ é denso no espaço das funções analítica em $\mathbb{C}$, temos,

$$
\begin{aligned}
\int K^{U}(z, w) f(w) e^{-\frac{|w|^{2}}{2}} d^{2} w & =e^{-\frac{|z|^{2}}{2}} \int \sum_{j=1}^{\infty} \phi_{j}^{U}(z) \overline{\phi_{j}^{U}(w)} \sum_{k=1}^{\infty} a_{k} \phi_{k}^{U}(w) d \nu(w) \\
& =e^{-\frac{|z|^{2}}{2}} \sum_{j, k=1}^{\infty} a_{k} \phi_{j}^{U}(z) \delta_{j k}=e^{-\frac{|z|^{2}}{2}} f(z) .
\end{aligned}
$$

O espaço $\mathcal{H}\left(\mathbb{C}, e^{-|z|^{2}}\right)$ é conhecido como espaço de Segal-Bargmann [35]. 
Prova do Teorema 33. A prova da igualdade (1.46) é imediata. Definimos

$$
K(z, w)=\varphi_{\alpha N}^{\prime}(z) K^{U}\left(\varphi_{\alpha N}(z), \varphi_{\alpha N}(w)\right) \overline{\varphi_{\alpha N}^{\prime}(w)}
$$

e seja $f(z) e^{-\frac{\left|\varphi_{\alpha N}(z)\right|^{2}}{2}} \in \mathcal{H}\left(\widetilde{\mathbb{C}}, e^{-\left|\varphi_{\alpha N}(z)\right|^{2}}\right)$. Então

$$
\begin{aligned}
& \int_{\widetilde{\mathbb{C}}} K(z, \widetilde{w}) f(\widetilde{w}) e^{-\frac{\left|\varphi_{\alpha N}(\widetilde{w})\right|^{2}}{2}} d^{2} \widetilde{w} \\
= & \int_{\widetilde{\mathbb{C}}} \varphi_{\alpha N}^{\prime}(z) K^{U}\left(\varphi_{\alpha N}(z), \varphi_{\alpha N}(\widetilde{w}) \overline{\varphi_{\alpha N}^{\prime}(\widetilde{w})} f(\widetilde{w}) e^{-\frac{\left|\varphi_{\alpha N}(\widetilde{w})\right|^{2}}{2}} d^{2} \widetilde{w} .\right.
\end{aligned}
$$

Fazendo uma mudança de variável $\widetilde{w}=\varphi_{\alpha N}^{-1}(w)$ temos que a expressão acima fica

$$
\int_{\mathbb{C}} \varphi_{\alpha N}^{\prime}(z) K^{U}\left(\varphi_{\alpha N}(z), w\right) \overline{\varphi_{\alpha N}^{\prime}\left(\varphi_{\alpha N}^{-1}(w)\right)} f\left(\varphi_{\alpha N}^{-1}(w)\right) e^{-\frac{\left|\varphi_{\alpha N}^{-1}\left(\varphi_{\alpha N}(w)\right)\right|^{2}}{2}}\left|\left(\varphi_{\alpha N}^{-1}\right)^{\prime}(w)\right|^{2} d^{2} w
$$
mas

$$
\varphi_{\alpha N}^{\prime}\left(\varphi_{\alpha N}^{-1}(w)\right)=\frac{1}{\left(\varphi_{\alpha N}^{-1}\right)^{\prime}(w)}
$$

e portanto a penúltima expressão fica

$$
\varphi_{\alpha N}^{\prime}(z) \int_{\mathbb{C}} K^{U}\left(\varphi_{\alpha N}(z), w\right) f\left(\varphi_{\alpha N}^{-1}(w)\right) e^{-\frac{|w|^{2}}{2}}\left(\varphi_{\alpha N}^{-1}\right)^{\prime}(w) d^{2} w
$$

Notemos que a função $f\left(\varphi_{\alpha N}^{-1}(w)\right) e^{-\frac{|w|^{2}}{2}} \varphi_{\alpha N}^{-1}(w)$ pertence a $\mathcal{H}\left(\mathbb{C}, e^{-|z|^{2}}\right)$ e, portanto, é reproduzida por $K^{U}$. Logo

$$
\begin{aligned}
\int_{\widetilde{\mathbb{C}}} K(z, w) f(w) e^{-\frac{|w|^{2}}{2}} d^{2} w & =\varphi_{\alpha N}^{\prime}(z) f\left(\varphi_{\alpha N}^{-1}\left(\varphi_{\alpha N}(z)\right)\right) e^{-\frac{\left|\varphi_{\alpha N}(z)\right|^{2}}{2}}\left(\varphi_{\alpha N}^{-1}\right)^{\prime}\left(\varphi_{\alpha N}(z)\right) \\
& =f(z) e^{-\frac{\left|\varphi_{\alpha N}(z)\right|^{2}}{2}}
\end{aligned}
$$

onde na última passagem usamos (4.19) para $w=\varphi_{\alpha N}(z)$.

Assim concluímos a universalidade para este ensembles neste sentido, visto que o núcleo reprodutor (1.45) é universal, pois independente do potencial $V_{\alpha}$.

Como vimos no Corolário 34, a relação entre $K^{U}$ e $S^{\alpha}$ se dá por uma transformação conforme linear, o que mostra uma estreita relação com a abordagem da seção anterior. Um argumento plausível sobre esta pequena diferença entre $K^{U}$ e $S^{\alpha}$ (que não é observada no ensemble unitário hermitiano) pode ser explicada heuristicamente pela diferença de dimensão na qual os autovalores moram. Por isso, podemos intuir que no caso hermitiano a influência da repulsão é muito mais relevante que no caso normal $\left(V_{\alpha}\right)$. Além disso, a disposição dos autovalores em equilíbrio pode mudar com a variação do parâmetro $\alpha$, devido aos diversos arranjos cristalinos estáveis no plano. No caso hermitiano (unidimensional) não temos esta diversidade. 


\subsection{Universalidade de G. Oas}

Esta noção de universalidade é empregada em [21] e [22], onde a abordagem utilizada para esta noção é inspirada no livro [23], que usa a seguinte medida de probabilidade para o ensemble de Ginibre:

$$
\widehat{P}_{N}^{G}\left(z_{1}, \cdots, z_{N}\right)=Z_{N}^{-1} \prod_{1 \leq i<j \leq N}\left|z_{i}-z_{j}\right|^{2} e^{-\sum_{i=1}^{N}\left|z_{i}\right|^{2}},
$$

o que induz a seguinte medida de probabilidade para o ensemble unitário normal

$$
\begin{aligned}
\widehat{P}_{N}\left(z_{1}, \cdots, z_{N}\right) & =Z_{N}^{-1} \prod_{1 \leq i<j \leq N}\left|z_{i}-z_{j}\right|^{2} e^{-\sum_{i=1}^{N} V\left(z_{i}\right)} \\
& =Z_{N}^{-1} e^{-\sum_{1 \leq i \neq j \leq N} \log \left|z_{i}-z_{j}\right|^{-1}-\sum_{i=1}^{N} V\left(z_{i}\right)}
\end{aligned}
$$

que difere de (1.21) e (1.22) apenas pela ausência do fator $N$ à frente da somatória. A escala $N$ à frente do potencial $V$ em (1.22) faz com que a energia de interação das cargas com o potencial e a energia de interação entre as cargas fiquem com a mesma ordem em $N$ e, portanto temos um argumento físico que justifica a escala dos autovalores de $M_{N}$ para que no limite $N \rightarrow \infty$ obtemos uma medida empírica (1.24) com suporte compacto no plano complexo (adensamento dos autovalores) como vimos no Capítulo 2. Assim, com a ausência da escala $N$ à frente da somatória como na equação (4.20), o uso desta torna-se mais natural quando estamos interessados em propriedades como probabilidade de um conjunto aberto não conter autovalores e espaçamento dos autovalores, pois como nesta caso a energia devido a repulsão das cargas (primeira somatória em (4.21)) é da ordem $N^{2}$ enquanto que a energia devido à interação do potencial externo com as cargas (segunda somatória em (4.21)) é ordem $N$, esperamos que o espaçamento médio dos autovalores permanece $O(1)$ quando $N \rightarrow \infty$.

Usando (4.20), podemos obter o seguinte Teorema:

Teorema 85 Seja

$$
\widehat{E}_{N}^{V_{\alpha}}(r)=\int \widehat{P}_{N}^{V_{\alpha}}\left(z_{1}, \cdots, z_{N}\right) \prod_{k=1}^{N} \chi_{\mathbb{R} \backslash \mathbb{B}_{0, r}}\left(z_{k}\right) d^{2} z_{k}
$$

com

$$
\mathbb{B}_{0, r}=\{z \in \mathbb{C}:|z|<r\}
$$

$e$

$$
V_{\alpha}(z)=|z|^{\alpha}
$$


Então

$$
\widehat{E}_{N}^{V_{\alpha}}(r)=\prod_{k=1}^{N} \frac{\Gamma\left(\frac{2 k}{\alpha}, r^{\alpha}\right)}{\Gamma\left(\frac{2 k}{\alpha}\right)}
$$

onde

$$
\mathbb{B}_{0, r}=\{z \in \mathbb{C}:|z|<r\}, \Gamma(x, r)=\int_{r}^{\infty} t^{x-1} e^{-t} d t \text { e } \Gamma(x)=\Gamma(x, 0)
$$

Prova. A prova segue exatamente os passos da prova do Teorema 30, trocando $P_{N}^{V_{\alpha}}$ por $\widehat{P}_{N}^{V_{\alpha}}$.

Observação 86 Notemos que (4.23) não é equivalente a (4.18). Isso mostra que a ausência do fator $N$ à frente da soma na equação (4.20) implica que o espaçamento médio dos autovalores é $O(1)$ em $N$, porém o espaçamento médio nesse caso depende de $r$ e de $\alpha$; já a escala devido à $g_{\alpha}$ feita para a obtenção de (4.18) é tal que o espaçamento médio entre os autovalores seja $O(1)$ em $N$ e, além disso, com espaçamento unitário em $s$ independente de $\alpha$. Usaremos esta nova escala apenas para comparar nossos resultados nesta escala com os resultados de outros autores que se utilizam desta escala.

Observação 87 Este Teorema pode ser enunciado de maneira mais geral da seguinte forma: Seja

$$
\widehat{E}_{N}^{V}(r)=\int \widehat{P}_{N}^{V}\left(z_{1}, \cdots, z_{N}\right) \prod_{k=1}^{N} \chi_{\mathbb{R} \backslash \mathbb{B}_{0, r}}\left(z_{k}\right) d^{2} z_{k}
$$

onde

$$
\mathbb{B}_{0, r}=\{z \in \mathbb{C}:|z|<r\}
$$

e $V: \mathbb{C} \rightarrow \mathbb{R}$ é um potencial como no Teorema 40,

$$
V(z) \equiv V_{C}(r), \quad z=r e^{i \theta}
$$

com $V_{C}$ um potencial convexo e crescente (hipóteses para garantir a existência de autovalores na vizinhança de $z=0)$. Então,

$$
E_{N}^{V}(r)=\prod_{k=1}^{N} \frac{\int_{r}^{\infty} r_{k}^{2 k-1} e^{-V_{C}\left(r_{k}\right)} d r_{k}}{\int_{0}^{\infty} r_{k}^{2 k-1} e^{-V_{C}\left(r_{k}\right)} d r_{k}}
$$

Esta prova segue exatamente os passos da prova do Teorema anterior até a equação (4.16).

Em [21] (equação 4.32) afirma-se que no ensemble normal, $\widehat{E}_{N}^{V}$ com $V(z)$ polinômio em $r=|z|$ na vizinhança da origem é dado por (para $r$ pequeno)

$$
\widehat{E}_{N}^{V}(r)=1-c_{V} r^{2}+O\left(r^{3}\right)
$$


onde $c_{V}>0$ depende apenas de $V$ para todo $N$. Este é o sentido de universalidade expresso em [22]: a probabilidade de um disco aberto centrado em zero de raio $r$ não conter autovalores é da forma (4.24) para todo polinômio $V(z)$ em $r=|z|$ para $r$ pequeno, onde $c_{V}$ pode depender de $V$.

Para

$$
V(z)=V_{\alpha}(z)=|z|^{\alpha}
$$

a constante $c_{V}$ pode ser calculada explicitamente, como já enunciado no Teorema 35.

Prova do Teorema 35. Escrevendo a série de Taylor até segunda ordem em $r$ para $\widehat{E}_{N}^{V_{\alpha}}$ dado por (4.23) temos

$$
\begin{gathered}
\widehat{E}_{N}^{V_{\alpha}}(r)=\widehat{E}_{N}^{V_{\alpha}}(0)+\widehat{E}_{N}^{V_{\alpha} \prime}(0) r+\widehat{E}_{N}^{V_{\alpha} \prime \prime}(0) \frac{r^{2}}{2}+O\left(r^{3}\right) \\
\widehat{E}_{N}^{V_{\alpha}}(0)=\prod_{k=1}^{N} \frac{\Gamma\left(\frac{2 k}{\alpha}, 0\right)}{\Gamma\left(\frac{2 k}{\alpha}\right)}=1
\end{gathered}
$$

e calculando as derivadas de $\widehat{E}_{N}^{V_{\alpha}}$ em zero temos

$$
\begin{aligned}
\left(\widehat{E}_{N}^{V_{\alpha}}\right)^{\prime}(r) & =\prod_{k=1}^{N} \frac{1}{\Gamma\left(\frac{2 k}{\alpha}\right)} \sum_{l=1}^{N} \Gamma^{\prime}\left(\frac{2 l}{\alpha}, r^{\alpha}\right) \prod_{m \neq l} \Gamma\left(\frac{2 m}{\alpha}, r^{\alpha}\right) \\
& =\prod_{k=1}^{N} \frac{1}{\Gamma\left(\frac{2 k}{\alpha}\right)} \sum_{l=1}^{N}\left(-\alpha e^{-r^{\alpha}} r^{2 l-1}\right) \prod_{m \neq l} \Gamma\left(\frac{2 m}{\alpha}, r^{\alpha}\right) \\
& \Rightarrow \widehat{E}_{N}^{V_{\alpha} \prime}(0)=0
\end{aligned}
$$

onde usamos

$$
\Gamma^{\prime}\left(\frac{2 l}{\alpha}, r^{\alpha}\right)=\frac{\partial}{\partial r} \int_{r^{\alpha}}^{\infty} t^{\frac{2 l}{\alpha}-1} e^{-t} d t=-\alpha e^{-r^{\alpha}} r^{2 l-1}
$$

De (4.26) temos

$$
\begin{aligned}
\left(\widehat{E}_{N}^{V_{\alpha}}\right)^{\prime \prime}(r)= & \prod_{k=1}^{N} \frac{1}{\Gamma\left(\frac{2 k}{\alpha}\right)} \sum_{l=1}^{N}\left(\left(\alpha e^{-r^{\alpha}} r^{2(l-1)}\left(1-2 l+\alpha r^{\alpha}\right)\right) \prod_{m \neq l} \Gamma\left(\frac{2 m}{\alpha}, r^{\alpha}\right)\right. \\
& \left.+\left(-\alpha e^{-r^{\alpha}} r^{2 l-1}\right) \sum_{\substack{m \neq l \\
\Gamma^{\prime}}}\left(\frac{2 m}{\alpha}, r^{\alpha}\right) \prod_{\substack{j \neq l \\
j \neq m}} \Gamma\left(\frac{2 j}{\alpha}, r^{\alpha}\right)\right) .
\end{aligned}
$$


Logo, o único termo não nulo na soma é tal que $l=1$, assim

$$
\begin{aligned}
\widehat{E}_{N}^{V_{\alpha} \prime \prime}(0) & =\prod_{k=1}^{N} \frac{1}{\Gamma\left(\frac{2 k}{\alpha}\right)}\left((\alpha(1-2 \cdot 1)) \prod_{m \neq 1} \Gamma\left(\frac{2 m}{\alpha}, 0\right)\right) \\
& =\prod_{k=1}^{N} \frac{-\alpha}{\Gamma\left(\frac{2 k}{\alpha}\right)} \prod_{m \neq 1} \Gamma\left(\frac{2 m}{\alpha}\right)=\frac{-\alpha}{\Gamma\left(\frac{2}{\alpha}\right)}
\end{aligned}
$$

e, portanto, (4.25) fica

$$
\begin{aligned}
\widehat{E}_{N}^{V_{\alpha}}(r) & =1+\frac{-\alpha}{\Gamma\left(\frac{2}{\alpha}\right)} \frac{r^{2}}{2}+O\left(r^{3}\right) \\
& =1-\frac{1}{\frac{2}{\alpha} \Gamma\left(\frac{2}{\alpha}\right)} r^{2}+O\left(r^{3}\right)
\end{aligned}
$$

como queríamos.

Porém isto não garante a universalidade da grandeza $\widehat{E}_{N}^{V_{\alpha}}(r)$ para todo $r$. Cabe agora a seguinte questão: já que o comportamento de $\widehat{E}_{N}^{V_{\alpha}}(r)$ para $r$ pequeno é universal (no sentido acima), será que isto também ocorre para $r$ grande? É sabido que para o ensemble de Ginibre $(\alpha=2)$ temos

$$
\widehat{E}_{N}^{V_{2}}(r) \approx e^{-\frac{r^{4}}{4}}
$$

para $N$ e $r$ grandes, com $N>r$ (veja [23]). Mais precisamente

$$
\log \widehat{E}_{N}^{V_{2}}(r)=-\frac{r^{4}}{4}-r^{2}(\log r+O(1))
$$

e, portanto, podemos generalizar este conceito de universalidade para $r$ grande como

$$
\log \widehat{E}_{N}^{V_{\alpha}}(r) \approx-c_{\alpha} r^{4}
$$

Veremos a seguir que este tipo de dependência no decaimento de $\widehat{E}_{N}^{V_{\alpha}}(r)$ para $r$ grande não ocorre, de forma que o expoente de $r$ no lado direito de (4.28) também deve depender de $\alpha$.

Lema 88 Seja,

$$
e_{n}(x)=\sum_{k=0}^{n-1} \frac{x^{k}}{k !}
$$

a série de potências da função exponencial truncada no n-ésimo termo. Então

$$
e^{-x} e_{n}(x)=\frac{\Gamma(n, x)}{\Gamma(n)}
$$

onde

$$
\Gamma(n, x)=\int_{x}^{\infty} t^{n-1} e^{-t} d t \text { e } \Gamma(n)=\Gamma(n, 0) .
$$


Prova. Derivando $e_{n}(x)$ notamos que

$$
\frac{d}{d x} e_{n}(x)=\sum_{k=1}^{n-1} \frac{x^{k-1}}{(k-1) !}=\sum_{j=0}^{n-2} \frac{x^{j}}{j !}=e_{n-1}(x) .
$$

Assim

$$
\begin{aligned}
\frac{d}{d x}\left(e^{-x} e_{n}(x)\right) & =-e^{-x} e_{n}(x)+e^{-x} e_{n-1}(x) \\
& =e^{-x}\left(-e_{n}(x)+e_{n-1}(x)\right) \\
& =-e^{-x} \frac{x^{n-1}}{(n-1) !} .
\end{aligned}
$$

Integrando os dois lados da igualdade acima e usando o Teorema Fundamental do Cálculo, obtemos

$$
\begin{aligned}
e^{-x} e_{n}(x) & =-\int_{x}^{\infty} \frac{d}{d t}\left(e^{-t} e_{n}(t)\right) d t \\
& =\int_{x}^{\infty} e^{-t} \frac{t^{n-1}}{(n-1) !} d t \\
& =\frac{\Gamma(n, x)}{\Gamma(n)}
\end{aligned}
$$

como queríamos.

Observação 89 Notamos que para $n$ grande a expressão

$$
e^{-x} e_{n}(x)=\int_{x}^{\infty} e^{-t} \frac{t^{n-1}}{(n-1) !} d t
$$

comporta-se essencialmente com a função

$$
\Theta(n-x)=\left\{\begin{array}{l}
1 \text { se } n>x \\
0 \text { se } n \leq x
\end{array}\right.
$$

Para ilustrar este fato, abaixo encontramos o gráfico da função (4.31) para $n=100$ e 200. 


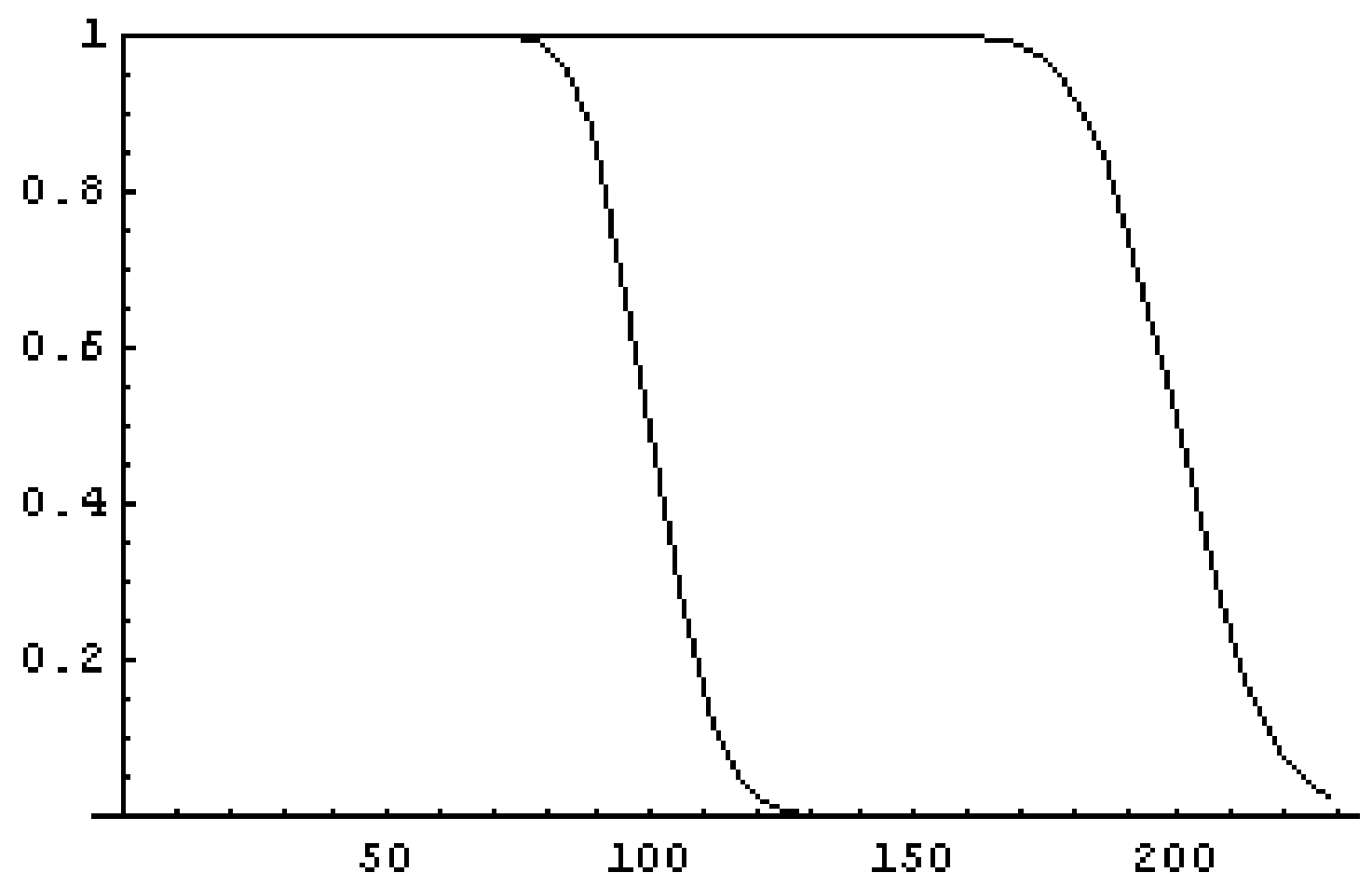

Provaremos a seguir que no caso particular $\alpha=1$ o decaimento em (4.27) para $r$ grande é substituído por $O\left(r^{2}\right)$.

prova do Teorema 36. Para $\alpha=1$ temos, de (4.23)

$$
\begin{aligned}
\log \widehat{E}_{N}^{V_{1}}(r) & =\log \prod_{k=1}^{N} \frac{\Gamma(2 k, r)}{\Gamma(2 k)} \\
& =\sum_{k=1}^{N} \log \frac{\Gamma(2 k, r)}{\Gamma(2 k)}
\end{aligned}
$$

e usando (4.29) obtemos

$$
\log \widehat{E}_{N}^{V_{1}}(r)=\sum_{k=1}^{N} \log \left(e^{-r} e_{2 k}(r)\right) .
$$

Da Observação 89, notamos que para $N$ grande, somente para $1 \leq k \lesssim r$ a soma acima 
recebe contribuições relevantes. Logo

$$
\begin{aligned}
\lim _{N \rightarrow \infty} \log \widehat{E}_{N}^{V_{1}}(r) & =\sum_{k=1}^{r} \log \left(e^{-r} e_{2 k}(r)\right) \\
& =\sum_{k=1}^{r} \log \left(e^{-r} \sum_{j=0}^{2 k-1} \frac{r^{j}}{j !}\right) \\
& =\sum_{k=1}^{r} \log \left(e^{-r} \frac{r^{2 k-1}}{(2 k-1) !}\left(1+O\left(\frac{1}{r}\right)\right)\right) \\
& =\sum_{k=1}^{r} \log \left(e^{-r} \frac{r^{2 k-1}}{(2 k-1) !}\right)+\sum_{k=1}^{r} \log \left(1+O\left(\frac{1}{r}\right)\right) \\
& =\sum_{k=1}^{r} \log \left(e^{-r} \frac{r^{2 k-1}}{(2 k-1) !}\right)+O(1)
\end{aligned}
$$

onde na antepenúltima passagem foi usado o termo dominante em $r$ de $e_{2 k}(r)$ (que é um polinômio em $r$ ).

Usando a fórmula da soma de Euler-MacLaurin

$$
\begin{aligned}
\sum_{k=1}^{N} f(k)= & \int_{1}^{N} f(k) d k+\frac{1}{2}(f(1)+f(N))+\sum_{k=1}^{m} \frac{B_{2 k}}{(2 k) !}\left(f^{(2 k-1)}(1)-f^{(2 k-1)}(N)\right) \\
& +\frac{N B_{2 m+2}}{(2 m+2) !} f^{(2 m+2)}(\gamma N)
\end{aligned}
$$

onde $f^{(2 m+2)}$ existe e é contínua para $1 \leq x \leq N, m$ e $\left.N=1,2 \cdots, \gamma \in\right] 0,1\left[\right.$ e $B_{k}$ são os números de Bernoulli de ordem $k$

$$
\frac{t}{e^{t}-1}=\sum_{k=0}^{\infty} B_{k} \frac{t^{k}}{k !}
$$

(veja [36] equação 4.8-10). Para $m=1$ e

$$
f(k) \equiv \log \left(e^{-r} \frac{r^{2 k-1}}{(2 k-1) !}\right)
$$

podemos escrever (4.32) como

$$
\begin{aligned}
\lim _{N \rightarrow \infty} \log \widehat{E}_{N}^{V_{\alpha}}(r)= & \int_{1}^{r} f(k) d k+\frac{1}{2}(f(1)+f(r)) \\
& +\frac{B_{2}}{(2) !}\left(f^{\prime}(1)+f \prime(r)\right)+\frac{r B_{4}}{(4) !} f^{(4)}(\gamma r)+O(1) .
\end{aligned}
$$


Assim, calcularemos cada termo da equação (4.32) separadamente. Para $k$ grande podemos escrever, usando a fórmula de Stirling

$$
\begin{aligned}
\log (2 k-1) ! & =\left(2 k-\frac{1}{2}\right) \log (2 k-1)-2 k+\overbrace{1+\frac{1}{2} \log (2 \pi)}^{c}+O\left(\frac{1}{k}\right) \\
& =\left(2 k-\frac{1}{2}\right) \log \left(2 k\left(1-\frac{1}{2 k}\right)\right)-2 k+c+O\left(\frac{1}{k}\right) \\
& =\left(2 k-\frac{1}{2}\right)\left(\log (2 k)+\log \left(1-\frac{1}{2 k}\right)\right)-2 k+c+O\left(\frac{1}{k}\right) \\
& =\left(2 k-\frac{1}{2}\right)\left(\log 2+\log k-\frac{1}{2 k}+O\left(\frac{1}{k^{2}}\right)\right)-2 k+c+O\left(\frac{1}{k}\right) \\
& =2 k \log k+2 k(\log 2-1)-\frac{1}{2} \log k+\widehat{c}+O\left(\frac{1}{k}\right)
\end{aligned}
$$

onde

$$
\widehat{c}=c-\frac{1}{2} \log 2-1=\frac{1}{2} \log \pi
$$

de forma que para $k$ grande, (4.33) pode ser escrita como

$$
\begin{aligned}
f(k) & =\log \left(e^{-r} \frac{r^{2 k-1}}{(2 k-1) !}\right) \\
& =-r+(2 k-1) \log r-\log (2 k-1) ! \\
& =-r+(2 k-1) \log r-2 k(\log k+\log 2-1)+\frac{1}{2} \log k-\widehat{c}+O\left(\frac{1}{k}\right) .
\end{aligned}
$$


Assim, os termos do lado direito de (4.34) podem ser estimados:

$$
\begin{aligned}
\int_{1}^{r} f(k) d k= & \int_{1}^{r}(-r+(2 k-1) \log r-2 k(\log k+\log 2-1) \\
& \left.+\frac{1}{2} \log k-\widehat{c}+O\left(\frac{1}{k}\right)\right) d k \\
= & -r^{2}+r+\left(r^{2}-r\right) \log r-r^{2} \log r+r^{2}\left(\frac{3}{2}-\log 2\right) \\
& +\frac{1}{2}(r \log r-r)-\widehat{c} r+O(\log r) \\
= & -\left(\log 2-\frac{1}{2}\right) r^{2}-\frac{1}{2} r \log r+O(r)
\end{aligned}
$$

por $(4.36)$

$$
\begin{aligned}
& f(r)=-r+(2 r-1) \log r-2 r(\log r+\log 2-1)+\frac{1}{2} \log r-\widehat{c}+O\left(\frac{1}{r}\right) \\
& =-(2 \log 2+1) r+O(\log r) \\
& f(1)=-r+\log r-2(\log 2-1)-\widehat{c}+O\left(\frac{1}{k}\right) \\
& =-r+O(\log r) \\
& f^{\prime}(k)=2 \log r-2(\log k+\log 2-1)-2+\frac{1}{2 k}+O\left(\frac{1}{k^{2}}\right) \Rightarrow \\
& f^{\prime}(r)=2 \log r-2(\log r+\log 2-1)-2+\frac{1}{2 r}+O\left(\frac{1}{r^{2}}\right) \\
& =-2(\log 2-1)-2+O\left(\frac{1}{r}\right) \\
& f^{\prime}(1)=2 \log r+O(1) \\
& f^{\prime \prime}(k)=\frac{2}{k}-\frac{1}{2 k^{2}}+O\left(\frac{1}{k^{3}}\right) \Rightarrow \\
& f^{\prime \prime}(k)=\frac{2}{k}-\frac{1}{2 k^{2}}+O\left(\frac{1}{k^{3}}\right) \Rightarrow f^{(4)}(k)=O\left(\frac{1}{k^{3}}\right) \text {. }
\end{aligned}
$$


Assim, substituindo (4.37), (4.38), (4.39), (4.40), (4.41) e (4.42) em (4.34) obtemos

$$
\lim _{N \rightarrow \infty} \log \widehat{E}_{N}^{V_{1}}(r)=-\left(\log 2-\frac{1}{2}\right) r^{2}-\frac{1}{2} r(\log r+O(1))
$$

como queríamos.

Logo, a partir do Teorema acima notamos que o decaimento de $\widehat{E}_{N}^{V_{\alpha}}$ depende de $\alpha$, pois para $\alpha=1$

$$
\lim _{N \rightarrow \infty} \log \widehat{E}_{N}^{V_{1}}(r)=-\left(\log 2-\frac{1}{2}\right) r^{2}-\frac{1}{2} r(\log r+O(1))
$$

e para $\alpha=2$

$$
\log \widehat{E}_{N}^{V_{2}}(r)=-\frac{r^{4}}{4}+-r^{2}(\log r+O(1))
$$

e, portanto, não temos universalidade neste sentido para o ensemble unitário normal, o que está de acordo com a seção anterior. 



\section{Capítulo 5}

\section{Conclusões}

Nesta Tese tratamos de forma rigorosa os seguintes aspectos do ensemble de matrizes normais:

1. A relação entre o potencial (que define o ensembles de matrizes normais) e a medida de equilíbrio associada a este ensemble é bem estabelecida na literatura (veja [27] por exemplo). Relacionamos o potencial diretamente com a medida de balayage associada à medida de equilíbrio deste ensemble (Teorema 19). Estabelecemos aqui uma relação entre as medidas de equilíbrio de ensembles normais unitários e ensembles hermitianos unitários através da medida de balayage. Encontramos explicitamente a medida de balayage associada ao ensemble unitário normal com potencial radialmente simétrico (em particular o ensemble normal de Ginibre) que coincide com a medida de equilíbrio do ensemble circular de Dyson (Teorema 21). Encontramos também uma fórmula explicita para a medida de balayage associada ao potencial de Elbau-Felder (Teorema 22), que no caso particular do ensemble normal de GinibreGirko relaciona a medida de balayage deste ensemble com a medida de equilíbrio do ensemble de Wigner no limite que o suporte desta medida de balayage tende para o eixo real (Teorema 23). Outro fato curioso que estabelece uma outra forma de relacionamento entre ensembles é que a simples projeção ortogonal da medida de equilíbrio do ensembles unitário normal radialmente simétricos coincide com a medida de equilíbrio do ensembles unitário hermitiano com potenciais proporcionais (Teorema 24).

2. Calculamos explicitamente o núcleo de um operador integral que contém toda a informação estatística do ensemble unitário normal radialmente simétrico (Teorema 25) e em particular, do potencial da forma monômio na parte radial (Corolário 26). 
Além disso, obtivemos uma expressão assintótica na ordem $N$ das matrizes do ensemble para o núcleo integral associado ao potencial da forma monômio na parte radial (Teorema 27). Este último teorema associado a uma propriedade que relaciona o núcleo integral e sua medida de equilíbrio correspondente gera um procedimento limite que define as escalas apropriadas para obtenção do núcleo integral assintótico (1.35).

3. Fazendo uso do cálculo assintótico do núcleo integral para a família de ensembles unitário normal definidos pelo potencial

$$
V_{\alpha}(z)=|z|^{\alpha},
$$

estendemos o sentido de universalidade usado por Deift para abordar o ensemble unitário hermitiano e concluímos que o limite do núcleo integral devidamente escalado $S^{\alpha}$ associado ao potencial (5.1) ainda depende de $\alpha$. Empregamos a teoria de espaços de Segal-Bargmann e mapas conformes para estabelecer uma relação simples entre o núcleo integral universal $K^{U}$ (independente de $V_{\alpha}$ ) e o núcleo integral escalado $S^{\alpha}$.

4. Em [21] afirma-se que a forma funcional da probabilidade de não encontrarmos autovalores nas proximidades da origem, em primeira ordem com a distância, não depende do potencial. Para potenciais radialmente simétrico da forma (5.1), nós provamos que de fato isso ocorre (Teorema 35). Uma tentativa de estender este resultado para grandes distâncias foi respondida negativamente pelo Teorema 36 .

Podemos citar alguns problemas em aberto:

1. No Teorema 23 obtivemos uma interpolação das medidas de equilíbrio do ensemble normal de Ginibre e do ensemble de Wigner através do ensemble normal de GinibreGirko. Seria interessante encontrarmos a mesma interpolação para os núcleos integrais associados a estes ensembles.

2. Encontrar um ensemble unitário normal que interpolasse as medidas de equilíbrio dos ensembles relacionados pelo Teorema 24. Desta forma possivelmente teríamos um Teorema análogo ao Teorema 23 para os ensembles de Ginibre e Wigner, além da questão anterior para este caso.

3. Obter o núcleo integral universal para outras famílias de potenciais e relacionar estes núcleos integrais entre si. 


\section{Apêndice A}

\section{Ensemble unitário normal}

\section{A.1 Álgebra linear e cálculo diferencial no plano com- plexo.}

O texto a seguir foi baseado principalmente na referência [37].

O plano complexo $\mathbb{C}$ coincide com o plano cartesiano $\mathbb{R}^{2}$ pela identificação usual

$$
\mathbb{C} \ni z=x+i y \equiv(x, y) \in \mathbb{R}^{2}
$$

Portanto, o plano complexo tem duas estruturas de espaço vetorial: uma como um espaço vetorial de dimensão 2 sobre $\mathbb{R}$ e outra como um espaço vetorial de dimensão 1 sobre $\mathbb{C}$.

Seja $\mathcal{L}_{\mathbb{R}}(\mathbb{C}, \mathbb{R})$ o espaço de todos os mapas $\mathbb{R}$-lineares de $\mathbb{C}$ em $\mathbb{R}$. Estes mapas também são chamados de formas lineares reais. Como $\{1, i\}$ forma a base real de $\mathbb{C}$, o par de formas

$$
d x: h \longmapsto \operatorname{Re} h \text { e } d y: h \longmapsto \operatorname{Im} h, h \in \mathbb{C}
$$

formam uma base de $\mathcal{L}_{\mathbb{R}}(\mathbb{C}, \mathbb{R})$.

Seja $\mathcal{L}_{\mathbb{R}}(\mathbb{C})$ o espaço de todos as formas reais lineares de $\mathbb{C}$ em $\mathbb{C}$. Este espaço vetorial é de dimensão quatro sobre $\mathbb{R}$ e 2 sobre $\mathbb{C}$. De fato, a inclusão $\mathbb{R} \subset \mathbb{C}$ nos permite considerar $\mathcal{L}_{\mathbb{R}}(\mathbb{C}, \mathbb{R})$ como um subespaço $\mathbb{R}$-linear de $\mathcal{L}_{\mathbb{R}}(\mathbb{C})$. Podemos decompor a forma $L \in \mathcal{L}_{\mathbb{R}}(\mathbb{C})$ em $L=\operatorname{Re} L+i \operatorname{Im} L$. Portanto, como um espaço vetorial real

$$
\mathcal{L}_{\mathbb{R}}(\mathbb{C})=\mathcal{L}_{\mathbb{R}}(\mathbb{C}, \mathbb{R}) \oplus i \mathcal{L}_{\mathbb{R}}(\mathbb{C}, \mathbb{R})
$$

onde $\oplus$ representa a soma direta de dois espaços vetoriais e, portanto, vemos que $\mathcal{L}_{\mathbb{R}}(\mathbb{C})$ tem dimensão 4 sobre $\mathbb{R}$. Além disso, qualquer base real de $\mathcal{L}_{\mathbb{R}}(\mathbb{C}, \mathbb{R})$ é uma base complexa de $\mathcal{L}_{\mathbb{R}}(\mathbb{C})$ e, por outro lado, qualquer base complexa de $\mathcal{L}_{\mathbb{R}}(\mathbb{C})$ que for um mapa a valores 
reais é uma base real de $\mathcal{L}_{\mathbb{R}}(\mathbb{C}, \mathbb{R})$. Em particular, $\{d x, d y\}$ é uma base complexa de $\mathcal{L}_{\mathbb{R}}(\mathbb{C})$.

Consideramos agora o subespaço complexo $\mathcal{L}_{\mathbb{C}}(\mathbb{C})$ de $\mathcal{L}_{\mathbb{R}}(\mathbb{C})$ formada pelas formas $\mathbb{C}$-lineares. Observe que uma forma

$$
L=P d x+Q d y \in \mathcal{L}_{\mathbb{R}}(\mathbb{C})
$$

é $\mathbb{C}$-linear se e somente se

$$
L(i h)=i L(h)
$$

para todo $h \in \mathbb{C}$. Escrevendo

$$
h=h_{1}+i h_{2} \operatorname{com} h_{1}, h_{2} \in \mathbb{R}
$$

encontramos

$$
L(i h)=L\left(-h_{2}+i h_{1}\right)=-P h_{2}+Q h_{1}
$$

enquanto

$$
i L(h)=i L\left(h_{1}+i h_{2}\right)=i P h_{1}+i Q h_{2} .
$$

Portanto, para que $L \in \mathcal{L}_{\mathbb{C}}(\mathbb{C})$ se e somente se $Q=i P$. Definindo a forma linear

$$
d z=d x+i d y
$$

então $d z \in \mathcal{L}_{\mathbb{C}}(\mathbb{C})$ e

$$
L=P d x+Q d y=P d z \in \mathcal{L}_{\mathbb{C}}(\mathbb{C})
$$

sempre que $Q=i P$. Em particular, $\mathcal{L}_{\mathbb{C}}(\mathbb{C})$ tem dimensão complexa 1 e dimensão real 2.

De maneira análoga a anterior, denotando por $\overline{\mathcal{L}_{\mathbb{C}}(\mathbb{C})}$ o subespaço complexo de $\mathcal{L}_{\mathbb{R}}(\mathbb{C})$ formada pelas formas que são $\mathbb{C}$-antilineares, isto é,

$$
L(\alpha h)=\bar{\alpha} L(h) \text { para } \alpha, h \in \mathbb{C} \text { e } L \in \overline{\mathcal{L}_{\mathbb{C}}(\mathbb{C})}
$$

podemos mostrar que

$$
L=P d x+Q d y=P d \bar{z} \in \mathcal{L}_{\mathbb{C}}(\mathbb{C})
$$

sempre que $Q=-i P$, onde

$$
d \bar{z}=d x-i d y
$$

A involução $z \longrightarrow \bar{z}$ pode ser estendida de $\mathbb{C}$ para $\mathcal{L}_{\mathbb{R}}(\mathbb{C})$, e ela troca $\mathcal{L}_{\mathbb{C}}(\mathbb{C})$ por $\overline{\mathcal{L}_{\mathbb{C}}(\mathbb{C})}$. Isso fornece uma decomposição em soma direta (como espaço vetorial real)

$$
\mathcal{L}_{\mathbb{R}}(\mathbb{C})=\mathcal{L}_{\mathbb{C}}(\mathbb{C}) \oplus \overline{\mathcal{L}_{\mathbb{C}}(\mathbb{C})}
$$


e novamente temos que $\{d z, d \bar{z}\}$ é também uma base complexa de $\mathcal{L}_{\mathbb{R}}(\mathbb{C})$. Assim podemos escrever um elemento $L \in \mathcal{L}_{\mathbb{R}}(\mathbb{C})$ como

$$
L=P d x+Q d y=A d z+B d \bar{z}
$$

com $P, Q, A$ e $B$ relacionados pelas equações

$$
\begin{array}{cc}
A=\frac{1}{2}(P-i Q), & B=\frac{1}{2}(P+i Q) \\
P=A+B, & Q=i(A-B) .
\end{array}
$$

Seja $\mathcal{B}\left(\mathbb{R}^{2} \times \mathbb{R}^{2}, \mathbb{C}\right)$ o espaço vetorial complexo dos mapas $\mathbb{R}$-bilinear de $\mathbb{R}^{2} \times \mathbb{R}^{2}$ em $\mathbb{C}$ (por simplicidade denotaremos apenas por $\mathcal{B}$ ). Assim, se $u, v \in \mathbb{R}^{2}$ e $B \in \mathcal{B}$ então

$$
u \mapsto B(u, v) \text { e } v \mapsto B(u, v) \text { são } \mathbb{R} \text {-lineares e } B(u, v)=-B(v, u) \text {. }
$$

Podemos gerar elementos de $\mathcal{B}$ pelo seguinte procedimento: seja $L_{1}, L_{2} \in \mathcal{L}_{\mathbb{R}}(\mathbb{C})$ e defina o produto exterior $L_{1} \wedge L_{2} \in \mathcal{B}$ por

$$
\left(L_{1} \wedge L_{2}\right)(u, v)=\operatorname{det}\left(\begin{array}{cc}
L_{1}(u) & L_{2}(u) \\
L_{1}(v) & L_{2}(v)
\end{array}\right)
$$

para $u, v \in \mathbb{R}^{2}$. Nesta notação o exemplo anterior está simplesmente $d x \wedge d y$.

Notemos que $\{d x \wedge d y\}$ é uma base complexa de $\mathcal{B}$ e, portanto, $\mathcal{B}$ é um espaço vetorial de dimensão complexa 1 . De fato, $d x \wedge d y \neq 0$ e para todo $B \in \mathcal{B}$ podemos escrever

$$
B=B((1,0),(0,1)) d x \wedge d y .
$$

Agora mostraremos que $\{d z \wedge d \bar{z}\}$ também é uma base de $\mathcal{B}$. Primeiro verificamos que

$$
\begin{aligned}
\mathcal{L}_{\mathbb{R}}(\mathbb{C}) \times \mathcal{L}_{\mathbb{R}}(\mathbb{C}) & \rightarrow \quad \mathcal{B} \\
\left(L_{1}, L_{2}\right) & \mapsto L_{1} \wedge L_{2}
\end{aligned}
$$

é também $\mathbb{R}$ bilinear e alternado. Isto prova a propriedade distributiva do produto exterior com respeito a soma e que $L_{1} \wedge L_{1}=0$ para todo $L_{1} \in \mathcal{L}_{\mathbb{R}}(\mathbb{C})$. Em particular

$$
\begin{gathered}
d x \wedge d x=d y \wedge d y=d z \wedge d z=d \bar{z} \wedge d \bar{z}=0, \\
d x \wedge d y=-d y \wedge d x
\end{gathered}
$$

e

$$
d z \wedge d \bar{z}=(d x+i d y) \wedge(d x-i d y)=-2 i d x \wedge d y=-d \bar{z} \wedge d z
$$

e, portanto, $\{d z \wedge d \bar{z}\}$ é também uma base de $\mathcal{B}$. 
Definição A.1 Uma 1-forma de classe $C^{k}, k \in \mathbb{N} \cup\{\infty\}$, em um aberto $\Omega \subset \mathbb{C}$ é uma função $\omega: \Omega \rightarrow \mathcal{L}_{\mathbb{R}}(\mathbb{C})$ de classe $C^{k}$ em $\Omega$, que pode ser escrita de maneira única como

$$
\omega=P d x+Q d y=A d z+B d \bar{z}
$$

onde $P, Q, A, B: \Omega \rightarrow \mathbb{C}$ são funções de classe $C^{k}$ em $\Omega$.

Definição A.2 Seja $f: \Omega \rightarrow \mathbb{C}$, uma função diferenciável em um aberto $\Omega \subset \mathbb{C}$. Então o diferencial df da função $f$ é uma 1-forma dada por

$$
d f=\frac{\partial f}{\partial x} d x+\frac{\partial f}{\partial y} d y
$$

na base $\{d x, d y\}$, onde

$$
\frac{\partial f}{\partial x} e \frac{\partial f}{\partial y}
$$

são as derivadas parciais de $f$ com respeito as variáveis $(x, y) \in \mathbb{R}^{2}$. Na base $\{d z, d \bar{z}\}$ temos

$$
d f=\frac{\partial f}{\partial z} d z+\frac{\partial f}{\partial \bar{z}} d \bar{z}
$$

onde

$$
\frac{\partial}{\partial z}=\frac{1}{2}\left(\frac{\partial}{\partial x}-i \frac{\partial}{\partial y}\right) e \frac{\partial}{\partial \bar{z}}=\frac{1}{2}\left(\frac{\partial}{\partial x}+i \frac{\partial}{\partial y}\right)
$$

Observação A.3 Se $f: \Omega \rightarrow \mathbb{C}$ é uma função holomorfa (anti-holomorfa), então

$$
d f=\frac{\partial f}{\partial z} d z \quad\left(d f=\frac{\partial f}{\partial \bar{z}} d \bar{z}\right)
$$

Definição A.4 Uma 2-forma de classe $C^{k}, k \in \mathbb{N} \cup\{\infty\}$, em um aberto $\Omega \subset \mathbb{C}$ é um mapeamento $\omega: \Omega \rightarrow \mathcal{B}$ de classe $C^{k}$ em $\Omega$, que pode ser escrita de maneira única como

$$
\omega=C d x \wedge d y=D d z \wedge d \bar{z}
$$

onde $C, D: \Omega \rightarrow \mathbb{C}$ são funções de classe $C^{k}$ em $\Omega$.

Definição A.5 Sejam $\Omega \subset \mathbb{C}$ um aberto, $\omega \subset \Omega$ um subconjunto (Lebesgue) mensurável de $\Omega$ e $f: \Omega \rightarrow \mathbb{C}$ uma função integrável sobre $\omega$ (com respeito à medida de Lebesgue $\left.d^{2} z\right)$. Assim

$$
f d x \wedge d y
$$

é uma 2-forma e definimos a integral de (A.1) como o número complexo

$$
\int_{\omega} f d x \wedge d y=\int_{\omega} f d^{2} z
$$

Observação A.6 Uma vez compreendido o formalismo do cálculo diferencial no plano complexo, basta generalizarmos este conceito quando olharmos funções ou formas definidas em $\Omega \subset \mathbb{C}^{N}$. Faremos isto na próxima Seção, usando a representação de determinante (ao invés do produto exterior) para estas formas. 


\section{A.2 Jacobiano de $\varphi$}

Lema A.7 Consideramos o espaço de Hilbert $\mathcal{H}$ formado pelas matrizes normais de ordem $N$ com produto escalar

$$
\left(A_{N}, B_{N}\right)=\operatorname{Tr}\left(A_{N}^{*} B_{N}\right)
$$

e definindo o mapa linear

$$
\vartheta A_{N}=U_{N}^{*} A_{N} U_{N}
$$

onde $U_{N}$ é uma matriz unitária de ordem $N$. Então

$$
|\operatorname{det} \vartheta|=1
$$

Prova. Pelas definições acima e pela propriedade cíclica do traço temos

$$
\left(\vartheta A_{N}, \vartheta B_{N}\right)=\operatorname{Tr}\left(U_{N}^{*} A_{N} U_{N} U_{N}^{*} B_{N} U_{N}\right)=\left(A_{N}, B_{N}\right)
$$

Logo $\vartheta$ é unitário e o Lema fica provado.

Corolário A.8 A identidade

$$
d \widetilde{M}_{N}=d M_{N}
$$

citada na Observação 12 é conseqüência imediata do Lema anterior, pois

$$
\widetilde{M}_{N}=\vartheta M_{N}=U_{N}^{*} M_{N} U_{N}
$$

e, portanto, o módulo do jacobiano da transformação

$$
\widetilde{M}_{N} \rightarrow M_{N}
$$

é igual a

$$
|\operatorname{det} \vartheta|=1
$$

Teorema A.9 Seja

$$
M_{N}=U_{N} \Lambda_{N} U_{N}^{*} \text { onde } U_{N} \in \mathcal{U}_{N}
$$

$\left(\mathcal{U}_{N}=\right.$ conjunto das matrizes Unitárias de ordem $\left.N\right), \Lambda_{N}=\operatorname{diag}\left\{z_{1}, \cdots, z_{N}\right\}$ e o mapa

$$
M_{N} \stackrel{\varphi}{\longrightarrow}\left(\Lambda_{N}, U_{N} \bmod \mathbb{T}^{N}\right)
$$

onde

$$
\mathbb{T}^{N}=\left\{\left(e^{i \theta_{1}}, \cdots, e^{i \theta_{N}}\right), 0 \leq \theta_{i}<2 \pi\right\}
$$


é o N-Toro. Então o Jacobiano da transformação (A.3) é dado por

$$
J(\varphi)=\left|\Delta_{N}\right|^{2} f(p)
$$

onde $f(p)$ é uma função positiva que depende apenas das $2 l=N^{2}-N$ coordenadas complexas $\left\{p_{i}, \bar{p}_{i}\right\}_{i=1}^{l}$ associadas aos autovetores normalizados $\left(U_{N} \bmod \mathbb{T}^{N}\right) e$

$$
\Delta_{N}=\prod_{1 \leq i<j \leq N}\left(z_{i}-z_{j}\right)
$$

Prova. Faremos explicitamente o cálculo da mudança de coordenadas $\left\{M_{i j}, \bar{M}_{i j}\right\}_{j \geq i=1}^{N}$ para as variáveis espectrais $\left\{z_{i}, \bar{z}_{i}\right\}_{i=1}^{N}$ e $\left\{p_{i}, \bar{p}_{i}\right\}_{i=1}^{l}$, baseado principalmente na referência [38]. Seja $A_{N}$ uma matriz de ordem $N$,

$$
\frac{\partial}{\partial z}=\frac{1}{2}\left(\frac{\partial}{\partial x}-i \frac{\partial}{\partial y}\right) \text { e } \frac{\partial}{\partial \bar{z}}=\frac{1}{2}\left(\frac{\partial}{\partial x}+i \frac{\partial}{\partial y}\right) \operatorname{com} z=x+i y
$$

Então denotaremos

$$
\frac{\partial A_{N}}{\partial z}=\left(\begin{array}{ccc}
\frac{\partial A_{1,1}}{\partial z} & \cdots & \frac{\partial A_{1, N}}{\partial z} \\
\vdots & \ddots & \vdots \\
\frac{\partial A_{N, 1}}{\partial z} & \cdots & \frac{\partial A_{N, N}}{\partial z}
\end{array}\right)_{N} \mathrm{e} \frac{\partial A_{N}}{\partial \bar{z}}=\left(\begin{array}{ccc}
\frac{\partial A_{1,1}}{\partial \bar{z}} & \cdots & \frac{\partial A_{1, N}}{\partial \bar{z}} \\
\vdots & \ddots & \vdots \\
\frac{\partial A_{N, 1}}{\partial \bar{z}} & \cdots & \frac{\partial A_{N, N}}{\partial \bar{z}}
\end{array}\right)_{N}
$$

De (A.2) temos, para as variáveis espectrais $\left\{p_{i}, \bar{p}_{i}\right\}_{i=1}^{l}$ associada aos autovetores de $M_{N}$ que

$$
\frac{\partial M_{N}}{\partial p_{\nu}}=\frac{\partial U_{N}}{\partial p_{\nu}} \Lambda_{N} U_{N}^{*}+U_{N} \Lambda_{N} \frac{\partial U_{N}^{*}}{\partial p_{\nu}} \text { para } 1 \leq \nu \leq l .
$$

Como $U_{N}^{*} U_{N}=1_{N}$ (matriz identidade de ordem $N$ ) temos que

$$
\frac{\partial U_{N}^{*}}{\partial p_{\nu}} U_{N}+U_{N}^{*} \frac{\partial U_{N}}{\partial p_{\nu}}=0_{N}
$$

e definindo

$$
S_{\nu}=U_{N}^{*} \frac{\partial U_{N}}{\partial p_{\nu}}
$$

concluímos que

$$
S_{\nu}=-S_{\nu}^{*}
$$

e, portanto,

$$
\begin{aligned}
U_{N}^{*} \frac{\partial M_{N}}{\partial p_{\nu}} U_{N} & =U_{N}^{*} \frac{\partial U_{N}}{\partial p_{\nu}} \Lambda_{N} U_{N}^{*} U_{N}+U_{N}^{*} U_{N} \Lambda_{N} \frac{\partial U_{N}^{*}}{\partial p_{\nu}} U_{N} \\
& =S_{\nu} \Lambda_{N}-\Lambda_{N} S_{\nu}=\left[S_{\nu}, \Lambda_{N}\right]
\end{aligned}
$$


Em termos de cada elemento temos

$$
\begin{aligned}
\left(U_{N}^{*} \frac{\partial M_{N}}{\partial p_{\nu}} U_{N}\right)_{j k} & =\sum_{l=1}^{N}\left(\left(S_{\nu}\right)_{j l}\left(\Lambda_{N}\right)_{l k}-\left(\Lambda_{N}\right)_{j l}\left(S_{\nu}\right)_{l k}\right) \\
& =\left(S_{\nu}\right)_{j k}\left(z_{k}-z_{j}\right) .
\end{aligned}
$$

Para $\bar{M}_{N}$ obtemos analogamente

$$
U_{N}^{*} \frac{\partial \bar{M}_{N}}{\partial p_{\nu}} U_{N}=\left[S_{\nu}, \bar{\Lambda}_{N}\right]
$$

e em termos de cada elemento temos

$$
\left(U_{N}^{*} \frac{\partial \bar{M}_{N}}{\partial p_{\nu}} U_{N}\right)_{j k}=\left(S_{\nu}\right)_{j k}\left(\bar{z}_{k}-\bar{z}_{j}\right)
$$

Novamente de (A.2) temos, para as variáveis espectrais $\left\{\bar{p}_{i}\right\}_{i=1}^{l}$

$$
\frac{\partial M_{N}}{\partial \bar{p}_{\nu}}=\frac{\partial U_{N}}{\partial \bar{p}_{\nu}} \Lambda_{N} U_{N}^{*}+U_{N} \Lambda_{N} \frac{\partial U_{N}^{*}}{\partial \bar{p}_{\nu}} \text { para } 1 \leq \nu \leq l
$$

novamente de $U_{N}^{*} U_{N}=1_{N}$ (matriz identidade de ordem $N$ ) temos que

$$
\frac{\partial U_{N}^{*}}{\partial \bar{p}_{\nu}} U_{N}+U_{N}^{*} \frac{\partial U_{N}}{\partial \bar{p}_{\nu}}=0_{N}
$$

e definindo

$$
\widehat{S}_{\nu}=U_{N}^{*} \frac{\partial U_{N}}{\partial \bar{p}_{\nu}}
$$

concluímos que

$$
\widehat{S}_{\nu}=-\widehat{S}_{\nu}^{*}
$$

e, portanto, analogamente como acima

$$
U_{N}^{*} \frac{\partial M_{N}}{\partial \bar{p}_{\nu}} U_{N}=\left[\widehat{S}_{\nu}, \Lambda_{N}\right]
$$

e em termos de cada elemento

$$
\left(U_{N}^{*} \frac{\partial M_{N}}{\partial \bar{p}_{\nu}} U_{N}\right)_{j k}=\left(\widehat{S}_{\nu}\right)_{j k}\left(z_{k}-z_{j}\right) .
$$

Para $\bar{M}_{N}$ obtemos

$$
\frac{\partial \bar{M}_{N}}{\partial \bar{p}_{\nu}}=\frac{\partial U_{N}}{\partial \bar{p}_{\nu}} \bar{\Lambda}_{N} U_{N}^{*}+U_{N} \bar{\Lambda}_{N} \frac{\partial U_{N}^{*}}{\partial \bar{p}_{\nu}} \text { para } 1 \leq \nu \leq l
$$

e procedendo como antes obtemos

$$
U_{N}^{*} \frac{\partial \bar{M}_{N}}{\partial \bar{p}_{\nu}} U_{N}=\left[\widehat{S}_{\nu}, \bar{\Lambda}_{N}\right]
$$


e em termos de cada elemento

$$
\left(U_{N}^{*} \frac{\partial \bar{M}_{N}}{\partial \bar{p}_{\nu}} U_{N}\right)_{j k}=\left(\widehat{S}_{\nu}\right)_{j k}\left(\bar{z}_{k}-\bar{z}_{j}\right) .
$$

Para a parte dos autovalores $\left\{z_{i}\right\}_{i=1}^{N}$ do espectro temos (veja (A.2)),

$$
U_{N}^{*} \frac{\partial M_{N}}{\partial z_{\nu}} U_{N}=\frac{\partial \Lambda_{N}}{\partial z_{\nu}}
$$

para $1 \leq \nu \leq N$. Em termos de cada elemento:

$$
\left(U_{N}^{*} \frac{\partial M_{N}}{\partial q_{\nu}} U_{N}\right)_{j k}=\delta_{\nu j} \delta_{j k} .
$$

Para $\bar{M}_{N}$ obtemos

$$
U_{N}^{*} \frac{\partial \bar{M}_{N}}{\partial z_{\nu}} U_{N}=\frac{\partial \bar{\Lambda}_{N}}{\partial z_{\nu}}=0_{N}
$$

para $1 \leq \nu \leq N$

Para as variáveis $\left\{\bar{z}_{i}\right\}_{i=1}^{N}$ temos

$$
U_{N}^{*} \frac{\partial M_{N}}{\partial \overline{z_{\nu}}} U_{N}=0_{N}
$$

e para $\bar{M}_{N}$

$$
U_{N}^{*} \frac{\partial \bar{M}_{N}}{\partial \bar{z}_{\nu}} U_{N}=\frac{\partial \bar{\Lambda}_{N}}{\partial \bar{z}_{\nu}}
$$

para $1 \leq \nu \leq N$. Em termos de cada elemento:

$$
\left(U_{N}^{*} \frac{\partial \bar{M}_{N}}{\partial \bar{z}_{\nu}} U_{N}\right)_{j k}=\delta_{\nu j} \delta_{j k}
$$

Doravante iremos representar a metade dos elementos independentes de uma matriz normal $A$ por um vetor coluna de $\frac{N^{2}+N}{2}$ da seguinte forma:

$$
\operatorname{vec} A=(A_{1,1}, A_{2,2}, \cdots, A_{N, N}, A_{1,2}, A_{1,3}, \cdots, \underbrace{A_{i, j}}_{i<j}, \cdots, A_{N-1, N})^{t} .
$$

Para não carregarmos a notação representaremos vec $A \operatorname{simplesmente~por~} A$. Assim, podemos denotar a matriz jacobiana de $\varphi$ pela matriz $\left(N^{2}+N\right) \times\left(N^{2}+N\right)$ (lembrando que $\left.l=\frac{N^{2}-N}{2}\right)$

$$
\left(\begin{array}{cccccccccccc}
\frac{\partial M_{N}}{\partial z_{1}} & \ldots & \frac{\partial M_{N}}{\partial z_{N}} & \frac{\partial M_{N}}{\partial p_{1}} & \ldots & \frac{\partial M_{N}}{\partial p_{l}} & \frac{\partial M_{N}}{\partial \bar{z}_{1}} & \ldots & \frac{\partial M_{N}}{\partial \bar{z}_{N}} & \frac{\partial M_{N}}{\partial \bar{p}_{1}} & \ldots & \frac{\partial M_{N}}{\partial \bar{p}_{l}} \\
\vdots & \ddots & \vdots & \vdots & \ddots & \vdots & \vdots & \ddots & \vdots & \vdots & \ddots & \vdots \\
\frac{\partial \bar{M}_{N}}{\partial z_{1}} & \ldots & \frac{\partial \bar{M}_{N}}{\partial z_{N}} & \frac{\partial \bar{M}_{N}}{\partial p_{1}} & \ldots & \frac{\partial \bar{M}_{N}}{\partial p_{l}} & \frac{\partial \bar{M}_{N}}{\partial \bar{z}_{1}} & \ldots & \frac{\partial \bar{M}_{N}}{\partial \bar{z}_{N}} & \frac{\partial \bar{M}_{N}}{\partial \bar{p}_{1}} & \ldots & \frac{\partial \bar{M}_{N}}{\partial \bar{p}_{l}}
\end{array}\right)
$$


(notamos que nesta representação cada coluna contém todos os $N^{2}+N$ elementos independentes da matriz normal $N \times N$ relativa a sua coluna).

Pelo Lema A.7 e das expressões (A.4), (A.6), (A.8) e (A.10) podemos escrever que

$$
\begin{aligned}
J(\varphi)= & \operatorname{det}\left(\begin{array}{cccccc}
\frac{\partial \Lambda_{N}}{\partial z_{1}} & \cdots & \frac{\partial \Lambda_{N}}{\partial z_{N}} & {\left[S_{1}, \Lambda_{N}\right]} & \cdots & {\left[S_{l}, \Lambda_{N}\right]} \\
\vdots & \ddots & \vdots & \vdots & \ddots & \vdots \\
\frac{\partial \bar{\Lambda}_{N}}{\partial z_{1}} & \cdots & \frac{\partial \bar{\Lambda}_{N}}{\partial z_{N}} & {\left[S_{1}, \bar{\Lambda}_{N}\right]} & \cdots & {\left[S_{l}, \bar{\Lambda}_{N}\right]} \\
\frac{\partial \Lambda_{N}}{\partial \bar{z}_{1}} & \cdots & \frac{\partial \Lambda_{N}}{\partial \bar{z}_{N}} & {\left[\widehat{S}_{1}, \Lambda_{N}\right]} & \cdots & \left.\left[\widehat{S}_{l}, \Lambda_{N}\right]\right) \\
\vdots & \ddots & \vdots & \vdots & \ddots & \vdots \\
\frac{\partial \bar{\Lambda}_{N}}{\partial \bar{z}_{1}} & \cdots & \frac{\partial \bar{\Lambda}_{N}}{\partial \bar{z}_{N}} & {\left[\widehat{S}_{1}, \bar{\Lambda}_{N}\right]} & \cdots & \left.\left[\widehat{S}_{l}, \bar{\Lambda}_{N}\right]\right) \mid
\end{array}\right.
\end{aligned}
$$

onde este pode ser escrito na forma de bloco (usando as expressões (A.5), (A.7), (A.9) e (A.11)) como

$$
J(\varphi)=\left|\operatorname{det}\left(\begin{array}{cccc}
1_{N} & 0 & 0 & 0 \\
0 & D S & 0 & D \widehat{S} \\
0 & 0 & 1_{N} & 0 \\
0 & \bar{D} S & 0 & \bar{D} \widehat{S}
\end{array}\right)\right|
$$

onde $1_{N}$ é a matriz identidade de ordem $N$ e $S, \widehat{S}$ e $D$ são matrizes de ordem $l$ tais que

$$
\begin{aligned}
& D S=\left(\begin{array}{ccc}
\left(S_{1}\right)_{1,2}\left(z_{2}-z_{1}\right) & \cdots & \left(S_{l}\right)_{1,2}\left(z_{2}-z_{1}\right) \\
\vdots & \ddots & \vdots \\
\left(S_{1}\right)_{N-1, N}\left(z_{N}-z_{N-1}\right) & \cdots & \left(S_{l}\right)_{N-1, N}\left(z_{N}-z_{N-1}\right)
\end{array}\right) \\
& D \widehat{S}=\left(\begin{array}{ccc}
\left(\widehat{S}_{1}\right)_{1,2}\left(z_{2}-z_{1}\right) & \cdots & \left(\widehat{S}_{l}\right)_{1,2}\left(z_{2}-z_{1}\right) \\
\vdots & \ddots & \vdots \\
\left(\widehat{S}_{1}\right)_{N-1, N}\left(z_{N}-z_{N-1}\right) & \cdots & \left(\widehat{S}_{l}\right)_{N-1, N}\left(z_{N}-z_{N-1}\right)
\end{array}\right), \\
& \bar{D} S=\left(\begin{array}{ccc}
\left(S_{1}\right)_{1,2}\left(\overline{z_{2}}-\overline{z_{1}}\right) & \cdots & \left(S_{l}\right)_{1,2}\left(\overline{z_{2}}-\overline{z_{1}}\right) \\
\vdots & \ddots & \vdots \\
\left(S_{1}\right)_{N-1, N}\left(\overline{z_{N}}-\overline{z_{N-1}}\right) & \cdots & \left(S_{l}\right)_{N-1, N}\left(\overline{z_{N}}-\overline{z_{N-1}}\right)
\end{array}\right) \\
& \bar{D} \widehat{S}=\left(\begin{array}{ccc}
\left(\widehat{S}_{1}\right)_{1,2}\left(\overline{z_{2}}-\overline{z_{1}}\right) & \cdots & \left(\widehat{S}_{l}\right)_{1,2}\left(\overline{z_{2}}-\overline{z_{1}}\right) \\
\vdots & \ddots & \vdots \\
\left(\widehat{S}_{1}\right)_{N-1, N}\left(\overline{z_{N}}-\overline{z_{N-1}}\right) & \cdots & \left(\widehat{S}_{l}\right)_{N-1, N}\left(\overline{z_{N}}-\overline{z_{N-1}}\right)
\end{array}\right),
\end{aligned}
$$


e

$$
D=\operatorname{diag}\left(z_{2}-z_{1}, z_{3}-z_{1}, \cdots, z_{N}-z_{N-1}\right)
$$

(notamos que $D$ possui o mesmo ordenamento que os termos não diagonais de (A.18)). Colocando em evidência cada fator $z_{i}-z_{j}, i>j$ que multiplica as linhas de $N+1$ à $\frac{N(N+1)}{2}$ e cada fator $\bar{z}_{i}-\bar{z}_{j}, i>j$ que multiplica as linhas de $\frac{N(N+3)}{2}$ à $N(N+1)$ podemos escrever

$$
\begin{aligned}
J(\varphi) & =\left|\prod_{1 \leq i<j \leq N}\left(z_{i}-z_{j}\right)\right|^{2}\left|\operatorname{det}\left(\begin{array}{cccc}
1_{N} & 0 & 0 & 0 \\
0 & S & 0 & \widehat{S} \\
0 & 0 & 1_{N} & 0 \\
0 & S & 0 & \widehat{S}
\end{array}\right)\right| \\
& =\left|\Delta_{N}\right|^{2} f(p)
\end{aligned}
$$

onde

$$
\Delta_{N}=\prod_{1 \leq i<j \leq N}\left(z_{i}-z_{j}\right)
$$

e

$$
f(p)=\left|\operatorname{det}\left(\begin{array}{cccc}
1_{N} & 0 & 0 & 0 \\
0 & S & 0 & \widehat{S} \\
0 & 0 & 1_{N} & 0 \\
0 & S & 0 & \widehat{S}
\end{array}\right)\right|
$$

que só dependem das coordenadas $\left\{p_{i}, \bar{p}_{i}\right\}_{i=1}^{l}$ e o Teorema fica provado. 


\section{Apêndice B}

\section{Prova do Teorema 40 com hipóteses restritas}

Teorema B.1 (Saff-Totik) Seja $V: \mathbb{C} \rightarrow \mathbb{R}$ um potencial tal que:

i. $V$ é contínuo em $\mathbb{C}$.

ii.

$$
\lim _{|z| \rightarrow \infty, z \in \mathbb{C}}(V(z)-2 \log |z|)=\infty
$$

Consideramos o problema variacional

$$
E^{V} \equiv \inf _{\mu \in \mathcal{M}(\mathbb{C})} I^{V}(\mu)
$$

onde $\mathcal{M}(\mathbb{C})$ é o conjunto de todas as medidas de probabilidade em $\mathbb{C}$,

$$
I^{V}(\mu) \equiv \int\left(V(z)+U^{\mu}(z)\right) d \mu(z)
$$

é a "energia eletrostática" associada à $\mu$ e

$$
U^{\mu}(z)=\int \log |z-w|^{-1} d \mu(w)
$$

o "potencial logarítmico" associada à $\mu$. Então as seguintes propriedades valem:

a. $E^{V}$ é finito.

b. O suporte $\mathbb{S}^{V}$ de $\mu^{V}$ é compacto.

c. Existe um único elemento $\mu^{V} \in \mathcal{M}(\mathbb{C})$ tal que

$$
E^{V}=I^{V}\left(\mu^{V}\right)
$$


d. Tomando

$$
F^{V} \equiv E^{V}-\frac{1}{2} \int V(z) d \mu^{V}(z)
$$

então a desigualdade

$$
U^{\mu^{V}}(z)+\frac{V(z)}{2} \geq F^{V}
$$

vale $\mu^{V}$ quase em toda parte em $\mathbb{C}$.

e. Para quase todo $z \in \mathbb{S}^{V}$

$$
U^{\mu^{V}}(z)+\frac{V(z)}{2}=F^{V} .
$$

\section{B.1 Prova do item a}

Lema B.2 A seguinte desigualdade:

$$
|z-w| \leq \sqrt{1+|z|^{2}} \sqrt{1+|w|^{2}}
$$

é satisfeita $\forall z, w \in \mathbb{C}$.

Prova. Subtraindo as identidades

$$
\begin{gathered}
\left(1+|z|^{2}\right)\left(1+|w|^{2}\right)=1+|z|^{2}+|w|^{2}+|w|^{2}|z|^{2} \\
|z-w|^{2}=|z|^{2}+|w|^{2}+2 \mathcal{R} e(z \bar{w})
\end{gathered}
$$

membro a membro teremos

$$
\left(1+|z|^{2}\right)\left(1+|w|^{2}\right)-|z-w|^{2}=1+2 \mathcal{R} e(z \bar{w})+|w|^{2}|z|^{2}=|1+z \bar{w}|^{2}
$$

de onde se conclui (B.2).

Teorema B.3 ( $E^{V}$ finito) Consideramos o funcional de energia

$$
I^{V}(\mu) \equiv \int\left(V(z)+U^{\mu}(z)\right) d \mu(z)=\iint k^{V}(z, w) d \mu(z) d \mu(w)
$$

onde

$$
k^{V}(z, w)=\log |z-w|^{-1}+\frac{1}{2} V(z)+\frac{1}{2} V(w)
$$

Então

$$
I^{V}(\cdot): \mathcal{M}(\mathbb{C}) \rightarrow(-\infty, \infty]
$$

é um mapa bem definido, e conseqüentemente

$$
I^{V}\left(\mu^{V}\right)>-\infty
$$

Além disso,

$$
I^{V}\left(\mu^{V}\right)<\infty
$$


Prova. Tomando o logaritmo da desigualdade (B.2) e multiplicando esta por -1 temos

$$
\log |z-w|^{-1} \geq-\frac{1}{2} \log \left(1+|z|^{2}\right)-\frac{1}{2} \log \left(1+|w|^{2}\right) .
$$

Somando em ambos os membros $\frac{1}{2} V(z)+\frac{1}{2} V(w)$, juntamente com a definição

$$
\kappa^{V}(z)=V(z)-\log \left(1+|z|^{2}\right)
$$

temos:

$$
k(z, w) \geq \frac{1}{2} \kappa(z)+\frac{1}{2} \kappa(w)
$$

Podemos notar de (B.5) e das hipóteses $i$. e $i i$. sobre $V$ que

$$
\kappa^{V}(z) \geq c_{V}>-\infty
$$

onde $c_{V}$ é uma constante que depende apenas do potencial $V$ e, portanto,

$$
k^{V}(z, w) \underset{(B .7)}{\stackrel{(B .6)}{\geq}} c_{V} \stackrel{(B .3)}{\Rightarrow} I^{V}(\mu)=\iint k^{V}(z, w) d \mu(z) d \mu(w) \stackrel{\int d \mu=1}{\geq} c_{V}>-\infty
$$

e fica provado, em particular, que $I^{V}\left(\mu^{V}\right)>-\infty$.

Para provarmos que

$$
E^{V}=I^{V}\left(\mu^{V}\right)=\inf _{\mu \in \mathcal{M}(\mathbb{C})} I^{V}(\mu)<\infty
$$

basta notar que tanto $\log |z-w|^{-1}$ quanto $V(z)$ são integráveis com respeito ao subconjunto das medidas contínuas de probabilidade com todos os momentos e, portanto, existem medidas de probabilidade em $\mathcal{M}(\mathbb{C})$ que satisfazem $I^{V}(\mu)<\infty$ e segue que $E^{V}<\infty$

\section{B.2 Prova do item b}

Teorema B.4 Seja $\mu^{V}$ uma medida de equilíbrio. Então o suporte $\mathbb{S}^{\mu^{V}}$ de $\mu^{V}$ é compacto.

Prova. Seja $D \subset \mathbb{C}$ tal que $\mu^{V}(D)>0$ e definimos

$$
\mu_{\varepsilon}^{V} \equiv \frac{1}{1+\varepsilon \mu^{V}(D)}\left(\mu^{V}+\left.\varepsilon \mu^{V}\right|_{D}\right)
$$


onde $\left.\mu^{V}\right|_{D}$ é a restrição de $\mu^{V}$ à $D$. Tomamos $0<\varepsilon<1$, pois assim $\mu_{\varepsilon}^{V}$ é uma medida de probabilidade. Temos que

$$
\begin{aligned}
I^{V}\left(\mu_{\varepsilon}^{V}\right) \stackrel{(B .3)}{=} & \frac{1}{\left(1+\varepsilon \mu^{V}(D)\right)^{2}} \iint k^{V}(z, w) d\left(\mu^{V}+\left.\varepsilon \mu^{V}\right|_{D}\right)(z) d\left(\mu^{V}+\left.\varepsilon \mu^{V}\right|_{D}\right)(w) \\
= & \frac{1}{\left(1+\varepsilon \mu^{V}(D)\right)^{2}}\left(I^{V}\left(\mu^{V}\right)+\left.2 \varepsilon \iint k^{V}(z, w) d \mu^{V}\right|_{D}(z) d \mu^{V}(w)\right. \\
& \left.+\left.\left.\varepsilon^{2} \iint k^{V}(z, w) d \mu^{V}\right|_{D}(z) d \mu^{V}\right|_{D}(w)\right) .
\end{aligned}
$$

Do Teorema B.3 segue que todas as integrais acima estão bem definidas. Como $\mu^{V}$ minimiza $I^{V}$,

$$
\left.\frac{d}{d \varepsilon} I^{V}\left(\mu_{\varepsilon}^{V}\right)\right|_{\varepsilon=0}=0
$$

De (B.9) e (B.10), temos

$$
\left.\iint\left(k^{V}(z, w)-I^{V}\left(\mu^{V}\right)\right) d \mu^{V}\right|_{D}(z) d \mu^{V}(w)=0
$$

pois $I^{V}\left(\mu^{V}\right)=E^{V} \in \mathbb{R}$ e

$$
\mu^{V}(D)=\left.\int d \mu^{V}\right|_{D}(z)
$$

Portanto,

$$
\begin{aligned}
& 0=\left.\iint\left(k^{V}(z, w)-I^{V}\left(\mu^{V}\right)\right) d \mu^{V}\right|_{D}(z) d \mu^{V}(w) \\
&\left.\stackrel{(B .6)}{\geq} \iint\left(\frac{1}{2} \kappa^{V}(z)+\frac{1}{2} \kappa^{V}(w)-I^{V}\left(\mu^{V}\right)\right) d \mu^{V}\right|_{D}(z) d \mu^{V}(w) \\
& \quad=\left.\frac{1}{2} \int\left(\kappa^{V}(z)+\int\left(\kappa^{V}(w)-2 I^{V}\left(\mu^{V}\right)\right) d \mu^{V}(w)\right) d \mu^{V}\right|_{D}(z)
\end{aligned}
$$

Novamente pelas hipóteses $i$. e $i i$. sobre $V(z)$ e de

$$
\kappa^{V}(z)=V(z)-\log \left(1+|z|^{2}\right)
$$

concluímos que existe um $M$ tal que $\kappa^{V}(z)+\int\left(\kappa^{V}(w)-2 I^{V}\left(\mu^{V}\right)\right) d \mu^{V}(w) \geq 1$ para 
$|z|>M$. Logo, tomando $D \subset\{z:|z| \geq M\}$ temos

$$
\begin{aligned}
0 & \geq\left.\frac{1}{2} \int\left(\kappa^{V}(z)+\int\left(\kappa^{V}(w)-2 I^{V}\left(\mu^{V}\right)\right) d \mu^{V}(w)\right) d \mu^{V}\right|_{D}(z) \\
& \geq\left.\frac{1}{2} \int d \mu^{V}\right|_{D}(z) \\
& >0
\end{aligned}
$$

pela hipótese sobre $\left.\mu^{V}\right|_{D}$. Assim a contradição é formada e concluímos que o suporte de $\mu^{V}$ deve estar contido no disco $\{z \in \mathbb{C}:|z| \leq M\}$.

\section{B.3 Prova do item c}

\section{B.3.1 Existência}

Teorema B.5 (Existência da medida de equilíbrio) Seja $I^{V}(\mu) \operatorname{como}$ em (B.3). Então existe uma medida de probabilidade $\mu=\mu^{V}$ tal que

$$
E^{V}=\inf _{\mu \in \mathcal{M}(\mathbb{C})} I^{V}(\mu)=I^{V}\left(\mu^{V}\right)
$$

Prova. A idéia da prova e construirmos uma seqüência de medidas de probabilidade com uma subseqüência que converge fracamente para uma medida de probabilidade $\mu$ e a prova do Teorema é concluída por uma desigualdade que implica $\mu=\mu^{V}$ (medida de probabilidade que satisfaz a equação (B.11)).

Seja $\left\{\mu_{n}\right\}_{n=1}^{\infty}$ uma seqüência de medidas de probabilidade tais que

$$
I^{V}\left(\mu_{n}\right) \leq E^{V}+\frac{1}{n}
$$

Notamos que essa seqüência está bem definida, pois $-\infty<E^{V}=\inf _{\mu \in \mathcal{M}(\mathbb{C})} I^{V}(\mu)<\infty$ pelo Teorema B.3. Também pelo Teorema B.3 temos que

$$
\begin{aligned}
E^{V}+\frac{1}{n} & \geq I^{V}\left(\mu_{n}\right) \\
& \geq \frac{1}{2} \iint\left(\kappa(z)+\kappa(w) d \mu_{n}(z) d \mu_{n}(w)\right. \\
& =\int \kappa(z) d \mu_{n}(z)
\end{aligned}
$$


Como $\kappa^{V}(z)=V(z)-\log \left(1+|z|^{2}\right)$, então para qualquer $b$ existe $M=M(b)$ tal que $\kappa^{V}(z)>b$ para qualquer $|z| \geq M$ (devido às hipóteses $i$. e $i i$. sobre $V(z)$ ). Assim

$$
\begin{aligned}
E^{V}+\frac{1}{n} \geq & \geq \kappa(z) d \mu_{n}(z)=\int_{|z|<M} \kappa(z) d \mu_{n}(z)+\int_{|z| \geq M} \kappa(z) d \mu_{n}(z) \\
\geq & c_{V} \overbrace{\int_{|z|<M} d \mu_{n}(z)}^{\leq 1}+b \int_{|z| \geq M} d \mu_{n}(z) \Rightarrow \\
& \int_{|z| \geq M} d \mu_{n}(z) \leq \frac{E^{V}+\frac{1}{n}-c_{V}}{b} \equiv \varepsilon(M) .
\end{aligned}
$$

Esta expressão mostra que na seqüência acima não há fuga de massa para o infinito $\left(\left\{\mu_{n}\right\}_{n=1}^{\infty}\right.$ é uma seqüência tight). Logo, pelo Teorema da seleção de Helly, existe uma medida de probabilidade $\mu$ e uma subseqüência $\left\{\mu_{n_{k}}\right\}_{k=1}^{\infty}$ de $\left\{\mu_{n}\right\}_{n=1}^{\infty}$ que converge fracamente para $\mu$.

Para mostrar que $\mu$ é a medida de equilíbrio $\mu^{V}$, basta mostrar a desigualdade

$$
\varliminf_{n \rightarrow \infty} I^{V}\left(\mu_{n}\right) \geq I^{V}(\mu)
$$

pois, se isso for verdade, teremos:

$$
I^{V}(\mu) \stackrel{(B .15)}{\leq} \varliminf_{n \rightarrow \infty} I^{V}\left(\mu_{n}\right) \stackrel{(B .12)}{\leq} \varlimsup_{n \rightarrow \infty}\left(E^{V}+\frac{1}{n}\right)=E^{V}=\inf _{\mu \in \mathcal{M}(\mathbb{C})} I^{V}(\mu)
$$

o que prova o Teorema. Então provaremos (B.15).

Para todo $L \in \mathbb{R}$ temos

$$
I^{V}\left(\mu_{n}\right)=\iint k^{V}(z, w) d \mu_{n}(z) d \mu_{n}(w) \geq \iint \min \left(k^{V}(z, w), L\right) d \mu_{n}(z) d \mu_{n}(w)
$$

Seja

$$
g_{L}(z, w) \equiv \min \left(k^{V}(z, w), L\right)
$$

Notamos que $g$ é contínua e limitada em $\mathbb{C} \times \mathbb{C}$. Seja também

$$
\varepsilon>0 \text { e } M=M(\varepsilon) \text { tal que } \int_{|z| \geq M} d \mu<\varepsilon
$$

(que existem pois $\mu$ é uma medida de probabilidade). Tomamos $f$ uma função contínua, limitada e de suporte compacto tal que:

1. $f(z)=1$ para $|z|<M$,

2. $0 \leq f(z) \leq 1$ para todo $z$, 
3. $f(z)=0$ para $|z| \geq M+1$.

Assim, pela definição de $f$ e pela equação (B.19) podemos escrever o lado esquerdo de (B.17) da seguinte forma:

$$
\begin{aligned}
& \iint g_{L}(z, w) d \mu_{n}(z) d \mu_{n}(w) \\
= & \iint(1+f(z)-f(z)+f(z) f(w)-f(z) f(w)) g_{L}(z, w) d \mu_{n}(z) d \mu_{n}(w) \\
= & \iint f(z) f(w) g_{L}(z, w) d \mu_{n}(z) d \mu_{n}(w) \\
& +\iint f(z)(1-f(w)) g_{L}(z, w) d \mu_{n}(z) d \mu_{n}(w) \\
& +\iint(1-f(z)) g_{L}(z, w) d \mu_{n}(z) d \mu_{n}(w) .
\end{aligned}
$$

Denotando as últimas três parcelas do último membro da equação acima por $I, I I$ e $I I I$ podemos escrever:

$$
\iint g_{L}(z, w) d \mu_{n}(z) d \mu_{n}(w) \equiv I+I I+I I I
$$

Notamos que:

$$
\begin{gathered}
|I I| \leq L \int d \mu_{n}(w) \int(1-f(z)) d \mu_{n}(z) \\
\underset{\operatorname{def} f}{\leq} L \cdot 1 \cdot \int_{|z| \geq M} d \mu_{n}(z) \stackrel{(B .19)}{\leq}\|g\|_{\infty} \varepsilon \\
\Rightarrow \varlimsup_{n \rightarrow \infty}|I I| \leq L \varepsilon
\end{gathered}
$$

de modo análogo podemos mostrar que

$$
\varlimsup_{n \rightarrow \infty}|I I I| \leq L \varepsilon
$$

Agora faremos estimativas semelhantes às duas equações acima para $I$. Como a função $f(z) f(w) g_{L}(z, w)$ é contínua, limitada e de suporte compacto, podemos aplicar para esta função o Teorema da aproximação polinomial de Weierstrass: dado $\varepsilon^{\prime}>0$ existe $N=$ $N\left(\varepsilon^{\prime}\right)$ e um polinômio a valores reais $p(z, w)$ tal que

$$
\left|f(z) f(w) g_{L}(z, w)-f(z) f(w) p(z, w)\right| \leq \varepsilon^{\prime},
$$


e, portanto,

$$
\begin{aligned}
I= & \iint f(z) f(w) g_{L}(z, w) d \mu_{n}(z) d \mu_{n}(w) \\
= & \iint f(z) f(w)\left(g_{L}(z, w)+p(z, w)-p(z, w)\right) d \mu_{n}(z) d \mu_{n}(w) \\
= & \iint f(z) f(w)\left(g_{L}(z, w)-p(z, w)\right) d \mu_{n}(z) d \mu_{n}(w) \\
& +\iint f(z) f(w) p(z, w) d \mu_{n}(z) d \mu_{n}(w) \\
= & I^{\prime}+I I^{\prime} .
\end{aligned}
$$

Por (B.23), notamos que

$$
I^{\prime} \leq \varepsilon^{\prime} \cdot 1 \cdot 1=\varepsilon^{\prime}
$$

e

$$
\begin{aligned}
\varlimsup_{n \rightarrow \infty} I I^{\prime} & =\varlimsup_{n \rightarrow \infty} \sum_{i, j} a_{i j} \int f(z) z^{i} d \mu_{n}(z) \int f(w) w^{j} d \mu_{n}(w) \\
& =\sum_{i, j} a_{i j} \int f(z) z^{i} d \mu(z) \int f(w) w^{j} d \mu(w) \\
& =\iint f(z) f(w) p(z, w) d \mu(z) d \mu(w) \\
& \left.\leq \int .23\right) \\
& \leq \int f(z) f(w) g(z, w) d \mu(z) d \mu(w)+\varepsilon^{\prime} \\
& \leq \iint g(z, w) d \mu(z) d \mu(w)+\varepsilon^{\prime} .
\end{aligned}
$$

Assim, tomando o $\underline{\lim }_{n \rightarrow \infty}$ à esquerda e o $\varlimsup_{n \rightarrow \infty}$ à direita de (B.20) temos, pelas estimativas feitas para $I$ (B.24,B.25) II (B.21)e $I I I$ (B.22) que:

$$
\begin{aligned}
\varliminf_{n \rightarrow \infty} \iint g(z, w) d \mu_{n}(z) d \mu_{n}(w) & \leq \varlimsup_{n \rightarrow \infty}(I+I I+I I I) \\
& \leq \iint f(z) f(w) g(z, w) d \mu(z) d \mu(w)+O\left(\varepsilon^{\prime}\right)+O(\varepsilon) \\
& \leq \iint g(z, w) d \mu(z) d \mu(w)+O\left(\varepsilon^{\prime}\right)+O(\varepsilon)
\end{aligned}
$$


pelas definições de $f$ e $M$. Logo

$$
\left|\underline{\varliminf_{n \rightarrow \infty}} \iint g_{L}(z, w) d \mu_{n}(z) d \mu_{n}(w)-\iint g_{L}(z, w) d \mu(z) d \mu(w)\right| \leq O(\varepsilon)+O\left(\varepsilon^{\prime}\right) .
$$

Assim, como $\varepsilon, \varepsilon^{\prime}>0$ são arbitrários e lembrando da definição de $g_{L}$ (B.18)

$$
g_{L}(z, w) \equiv \min \left(k^{V}(z, w), L\right)
$$

temos que

$$
\varliminf_{n \rightarrow \infty} \iint \min \left(k^{V}(z, w), L\right) d \mu_{n}(z) d \mu_{n}(w)=\iint \min \left(k^{V}(z, w), L\right) d \mu(z) d \mu(w)
$$

mas tomando o $\underline{\lim _{n \rightarrow \infty}}$ de (B.17) segue

$$
\begin{aligned}
\varliminf_{n \rightarrow \infty} I^{V}\left(\mu_{n}\right) & \geq \quad \underline{\lim }_{n \rightarrow \infty} \iint \min \left(k^{V}(z, w), L\right) d \mu_{n}(z) d \mu_{n}(w) \\
& \stackrel{(B .26)}{=} \iint \min \left(k^{V}(z, w), L\right) d \mu(z) d \mu(w) .
\end{aligned}
$$

Agora, como $g_{L}$ pode se vista como uma seqüência de funções $\left\{g_{L}\right\}_{L=1}^{\infty}$ que são monótonas crescentes e que convergem, quando $L \rightarrow \infty$ para $k(z, w)$, fazendo $L \rightarrow \infty$ e usando o Teorema da convergência monótona temos que

$$
\begin{aligned}
\varliminf_{n \rightarrow \infty} I^{V}\left(\mu_{n}\right) & \geq \lim _{L \rightarrow \infty} \iint \min \left(k^{V}(z, w), L\right) d \mu(z) d \mu(w) \\
& =\iint k^{V}(z, w) d \mu(z) d \mu(w)=I^{V}(\mu)
\end{aligned}
$$

e com isso provamos (B.15) e, conseqüentemente, o Teorema pela equação (B.16).

\section{B.3.2 Unicidade.}

Teorema B.6 A medida de equilíbrio $\mu^{V}$ tem "energia potencial" e "energia de interação" ambas finitas. Mais precisamente,

$$
0 \leq \int V(z) d \mu^{V}(z)<\infty e-\infty<\iint \log |z-w|^{-1} d \mu^{V}(z) d \mu^{V}(w)<\infty
$$

Prova. Sabemos pelo Teorema B.3 que

$$
-\infty<E^{V}=\int V(z) d \mu^{V}(z)+\iint \log |z-w|^{-1} d \mu^{V}(z) d \mu^{V}(w)<\infty
$$


e, portanto, basta mostrar que

$$
c_{V} \leq \int V(z) d \mu^{V}(z)<\infty
$$

De fato, como $V$ é contínuo em $\mathbb{C}$ concluímos que existe uma constante $c_{V} \in \mathbb{R}$ tal que

$$
c_{V} \leq V(z)
$$

Assim, como $\mu^{V}$ tem suporte compacto temos, integrando ambos os membros da equação acima com respeito à $d \mu^{V}$

$$
c_{V}=\int_{\mathbb{S}^{\mu} V} c_{V} d \mu^{V}(z) \leq \int_{\mathbb{S}^{\mu}} V(z) d \mu^{V}(z) \leq \sup _{z \in \mathbb{S}^{\mu} V} V(z) \overbrace{\int_{\mathbb{S}^{\mu} V} d \mu^{V}(z)}^{=1}<\infty
$$

como queríamos.

Definição B.7 Uma função u $: \mathbb{D} \subset \mathbb{C} \rightarrow \mathbb{R}$ é harmônica em $\mathbb{D}$ se u e suas duas primeiras derivadas parciais são contínuas em $\mathbb{D}$ e satisfaz a equação de laplace

$$
\Delta u=0
$$

onde

$$
\Delta=\partial_{x}^{2}+\partial_{y}^{2}=4 \partial_{z} \partial_{\bar{z}}
$$

Observação B.8 (Propriedade do valor médio) Se u é harmônica no disco aberto

$$
\mathbb{D}=\{z \in \mathbb{C}:|z-a|<r\}
$$

e contínua em $\overline{\mathbb{D}}$, então

$$
u(a)=\frac{1}{2 \pi} \int_{-\pi}^{\pi} u\left(a+r e^{i \theta}\right) d \theta .
$$

Usaremos este resultado sem demonstração ([24] pág. 7).

Lema B.9 Para cada $r>0$,

$$
\frac{1}{2 \pi} \int_{-\pi}^{\pi} \log \frac{1}{\mid z-r e^{i \theta \mid}} d \theta=\left\{\begin{array}{l}
\log \frac{1}{r} \text { se }|z| \leq r \\
\log \frac{1}{|z|} \text { se }|z| \geq r
\end{array}\right.
$$


Prova. Esta fórmula para $|z|>r$ é obtida notando que $\log \frac{1}{|z-w|}$ é uma função harmônica como função de $w$ para $|w| \leq r$ e conseqüentemente podemos aplicar a propriedade do valor médio para $w=0$. Para $|z|<r$ temos por um argumento similar:

$$
\begin{aligned}
\frac{1}{2 \pi} \int_{-\pi}^{\pi} \log \frac{1}{\left|z-r e^{i \theta}\right|} d \theta & =\frac{1}{2 \pi} \int_{-\pi}^{\pi} \log \frac{1}{\left|z e^{-i \theta}-r\right|} d \theta \\
& =\frac{1}{2 \pi} \int_{-\pi}^{\pi} \log \frac{1}{\left|\bar{z} e^{i \theta}-r\right|} d \theta \\
& =\log \frac{1}{r}
\end{aligned}
$$

Finalmente para $|z|=r$ temos, pelo cálculo acima e pelo Teorema da convergência dominada

$$
\begin{aligned}
\frac{1}{2 \pi} \int_{-\pi}^{\pi} \log \frac{1}{\mid z-r e^{i \theta \mid}} d \theta & =\lim _{\rho \rightarrow r} \frac{1}{2 \pi} \int_{-\pi}^{\pi} \log \frac{1}{\mid z-\rho e^{i \theta \mid}} d \theta \\
& =\log \frac{1}{r}
\end{aligned}
$$

Definição B.10 (Medida com sinal) Sejam $\mu_{1}$ e $\mu_{2}$ duas medidas de Borel positivas e finitas. Então uma medida de Borel com sinal finita pode ser definida como

$$
\mu_{s}=\mu_{1}-\mu_{2}
$$

e sendo $f$ uma função integrável com respeito a $|\mu|=\mu_{1}+\mu_{2}$, então

$$
\int f d \mu_{s}=\int f d \mu_{1}-\int f d \mu_{2}
$$

Lema B.11 Seja $\mu_{s}=\mu_{1}-\mu_{2}$ uma medida de Borel com sinal finita e com suporte compacto e massa total zero (ou seja, $\int d \mu_{s}=0$ ). Suponhamos ainda que $\mu_{1}$ e $\mu_{2}$ tenham "energia logarítmica" finita:

$$
\int U^{\mu_{i}}(z) d \mu_{i}(z)=\iint \log |z-w|^{-1} d \mu_{i}(w) d \mu_{i}(z)<\infty \text { para } i=1,2 .
$$

Então a "energia logarítmica" de $\mu_{s}$ é não negativa

$$
\iint \log |z-w|^{-1} d \mu_{s}(z) d \mu_{s}(w)=\frac{1}{2 \pi} \int_{\mathbb{C}}\left(\int \frac{1}{|t-z|} d \mu_{s}(z)\right)^{2} d^{2} t \geq 0
$$

e é zero se e somente se $\mu_{s} \equiv 0$. 
Prova. Sejam $z$ e $w$ dois pontos do suporte de $\mu_{s}$ e para $R$ grande consideramos a integral

$$
I=\frac{1}{2 \pi} \int_{|t| \leq R} \frac{1}{|t-z||t-w|} d^{2} t
$$

Fazendo a mudança de variável

$$
t \rightarrow t+w, t=r e^{i \theta}
$$

obtemos

$$
\begin{aligned}
I & =\frac{1}{2 \pi} \int_{|t| \leq R} \frac{1}{|t+w-z||t|} d^{2} t+O\left(\frac{1}{R}\right) \\
& =\frac{1}{2 \pi} \int_{0}^{R} \int_{0}^{2 \pi} \frac{1}{\left|r e^{i \theta}-\right| z-w||} d r d \theta+O\left(\frac{1}{R}\right)
\end{aligned}
$$

onde $O\left(\frac{1}{R}\right)$ é uniforme em $z, w$ e no último passo usamos a simetria da integral. Com $x=|z-w|$ e $u=\frac{r}{x}$ temos

$$
\begin{aligned}
I & =\frac{1}{2 \pi} \int_{0}^{R} \int_{0}^{2 \pi} \frac{1}{\left|r e^{i \theta}-x\right|} d r d \theta+O\left(\frac{1}{R}\right) \\
& =\frac{1}{2 \pi} \int_{0}^{\frac{R}{x}} \int_{0}^{2 \pi} \frac{1}{\mid 1-u e^{i \theta \mid}} d u d \theta+O\left(\frac{1}{R}\right) \\
& =c_{1}+\int_{2}^{\frac{R}{x}}\left(\frac{1}{u}+O\left(\frac{1}{u^{2}}\right)\right) d u+O\left(\frac{1}{R}\right) \\
& =c_{2}+\log R-\log x+O\left(\frac{1}{R}\right) \\
& =c_{2}+\log R+\log |z-w|^{-1}+O\left(\frac{1}{R}\right)
\end{aligned}
$$

onde $c_{1}$ e $c_{2}$ são constantes. Assim, de (B.30) e como $\mu_{s}(\mathbb{C})=0$ obtemos

$$
\begin{aligned}
& \iint \log |z-w|^{-1} d \mu_{s}(z) d \mu_{s}(w) \\
= & \iint\left(I-c_{2}-\log R+O\left(\frac{1}{R}\right)\right) d \mu_{s}(z) d \mu_{s}(w) \\
= & \iint\left(\frac{1}{2 \pi} \int_{|t| \leq R} \frac{1}{|t-z||t-w|} d^{2} t\right) d \mu_{s}(z) d \mu_{s}(w) \\
= & \frac{1}{2 \pi} \int_{|t| \leq R}\left(\int \frac{1}{|t-z|} d \mu_{s}(z)\right)^{2} d^{2} t
\end{aligned}
$$


onde a justificativa do uso do Teorema de Fubini é dada a seguir: Seja $\left|\mu_{s}\right|$ a variação total de $\mu_{s}$ e $\| \mu_{s}||=\left|\mu_{s}\right|(\mathbb{C}) \neq 0$. Usando (B.30) para $\left|\mu_{s}\right|$ obtemos

$$
\begin{aligned}
& \iint \log |z-w|^{-1} d\left|\mu_{s}\right|(z) d\left|\mu_{s}\right|(w) \\
= & \iint\left(I-c_{2}-\log R+O\left(\frac{1}{R}\right)\right) d\left|\mu_{s}\right|(z) d\left|\mu_{s}\right|(w) \\
= & -\left.|| \mu_{s}\right|^{2} \log R+\frac{1}{2 \pi} \int_{|t| \leq R}\left(\int \frac{1}{|t-z|} d\left|\mu_{s}\right|(z)\right)^{2} d^{2} t+c_{3}+O\left(\frac{1}{R}\right)
\end{aligned}
$$

onde $c_{3}$ é uma constante. A expressão acima mostra que

$$
\int \frac{1}{|t-z|} d\left|\mu_{s}\right|(z)
$$

existe para quase todo $t$. Além disso

$$
\iiint_{|t| \leq R} \frac{1}{|t-z||t-w|} d^{2} t d\left|\mu_{s}\right|(z) d\left|\mu_{s}\right|(w)
$$

é finito. Isso mostra que a ordem da integração pode ser trocada em (B.31). Logo tomando $R \rightarrow \infty$ em (B.31) obtemos

$$
\iint \log |z-w|^{-1} d \mu_{s}(z) d \mu_{s}(w)=\frac{1}{2 \pi} \int_{\mathbb{C}}\left(\int \frac{1}{|t-z|} d \mu_{s}(z)\right)^{2} d^{2} t
$$

o que prova (B.29). Quando ocorre a igualdade em (B.29), a integral

$$
\int \frac{1}{|t-z|} d \mu_{s}(z)=0
$$

para quase todo $t$; mas sendo contínua para $t$ grande, podemos concluir que a integral se anula fora de um conjunto compacto. Então, para $t=r e^{i \theta}$

$$
\begin{aligned}
\frac{1}{|z-t|} & =r^{-1}\left(1-\frac{z}{r} e^{-i \theta}\right)^{-\frac{1}{2}}\left(1-\frac{\bar{z}}{r} e^{i \theta}\right)^{-\frac{1}{2}} \\
& =r^{-1} \sum_{m=0}^{\infty} \sum_{n=0}^{\infty}\left(-\frac{1}{r}\right)^{m+n}\left(\begin{array}{c}
-\frac{1}{2} \\
m
\end{array}\right)\left(\begin{array}{c}
-\frac{1}{2} \\
n
\end{array}\right) z^{m} \bar{z}^{n} e^{-i(m-n) \theta}
\end{aligned}
$$

e para $r$ grande, $k \in \mathbb{N}$ e devido a identidade

$$
\frac{1}{2 \pi} \int_{-\pi}^{\pi} e^{-i(k+m-n) \theta} d \theta=\delta_{n, m+k}
$$


segue de (B.33)

$$
\begin{aligned}
0 & =\frac{1}{2 \pi} \int_{-\pi}^{\pi} e^{-i k \theta} \int \frac{1}{\mid z-r e^{i \theta \mid}} d \mu_{s}(z) d \theta \\
& =\sum_{m=0}^{\infty}(-1)^{k} r^{-(2 m+k+1)}\left(\begin{array}{c}
-\frac{1}{2} \\
m
\end{array}\right)\left(\begin{array}{c}
-\frac{1}{2} \\
n
\end{array}\right) \int z^{m z^{m+k}} d \mu_{s}(z)
\end{aligned}
$$

Assim devemos ter

$$
\int z^{m} \bar{z}^{m+k} d \mu_{s}(z)=0
$$

para todo $m, k \geq 0$. Como os monômios $\left\{z^{m} \bar{z}^{n}\right\}_{m, n=0}^{\infty}$ formam uma base do espaço das funções contínuas definidas no suporte de $\mu_{s}$. Portanto, concluímos

$$
\int f d \mu_{s}=0
$$

para toda função $f$ contínua, concluindo que (B.29) atinge a igualdade se e somente se $\mu_{s}=0$.

Teorema B.12 (Unicidade da medida de equilíbrio) A medida de equilíbrio $\mu^{V}$

$$
E^{V}=\inf _{\mu \in \mathcal{M}(\mathbb{C})} I^{V}(\mu)=I^{V}\left(\mu^{V}\right)
$$

é única.

Prova. Sejam $\mu^{V}$ e $\widetilde{\mu}$ duas medidas de equilíbrio distintas e seja também $\mu_{s}=\widetilde{\mu}-\mu^{V}$ uma medida com sinal finita. Notamos que $\mu_{s}$ tem suporte compacto e média zero e que todas as integrais relevantes que possam envolver $\mu^{V}$ e $\widetilde{\mu}$ são finitas (pelo Corolário B.6). Claramente, a combinação convexa das medidas de equilíbrio como

$$
\mu_{t}=t \widetilde{\mu}+(1-t) \mu^{V}=\mu^{V}+t\left(\widetilde{\mu}-\mu^{V}\right)=\mu^{V}+t \mu_{s}, t \in[0,1]
$$

é uma medida de probabilidade e, pela definição de $I^{V}(\mu)$ e de $U^{\mu}(z)$, temos

$$
\begin{aligned}
I^{V}\left(\mu_{t}\right)= & \int\left(V(z)+U^{\mu_{t}}(z)\right) d \mu_{t}(z) \\
= & \int V(z) d \mu_{t}(z)+\iint \log |z-w|^{-1} d \mu_{t}(w) d \mu_{t}(z) \\
= & I^{V}\left(\mu^{V}\right)+t \int\left(V(z)+2 \int \log |z-w|^{-1} d \mu^{V}(w)\right) d \mu_{s}(z) \\
& +t^{2} \iint \log |z-w|^{-1} d \mu_{s}(z) d \mu_{s}(w)
\end{aligned}
$$


Como $\mu_{s}$ é uma medida com sinal de média zero e suporte compacto, pelo Lema B.11 temos que a função $I^{V}\left(\mu_{t}\right)$ como função de $t$ é convexa. Para $0 \leq t \leq 1$ temos:

$$
\begin{aligned}
E^{V} & \leq I^{V}\left(\mu_{t}\right)=I^{V}\left(t \widetilde{\mu}+(1-t) \mu^{V}\right) \\
& \leq t I^{V}(\widetilde{\mu})+(1-t) I^{V}\left(\mu^{V}\right)=t E^{V}+(1-t) E^{V}=E^{V}
\end{aligned}
$$

pela desigualdade de Jensen e, portanto, concluímos que $I^{V}\left(\mu_{t}\right)=E^{V}$ é uma constante como função de $t$. Em particular

$$
\begin{aligned}
0 & =\frac{1}{2} \frac{d^{2}}{d t^{2}} I^{V}\left(\mu_{t}\right) \\
& =\iint \log |z-w|^{-1} d \mu_{s}(z) d \mu_{s}(w) \\
& =\frac{1}{2 \pi} \int_{\mathbb{C}}\left(\int \frac{1}{|t-z|} d \mu_{s}(z)\right)^{2} d^{2} t
\end{aligned}
$$

onde na última passagem foi usado (B.29). Isso implica que

$$
\mu_{s}=0 \Rightarrow \widetilde{\mu}=\mu^{V}
$$

novamente pelo Lema B.11. O Teorema fica provado por absurdo.

\section{B.4 Prova do item d}

Teorema B.13 Seja V como no Teorema B.1 e $U^{\mu^{V}}$ o potencial logarítmico associado à medida de equilíbrio $\mu^{V}$. Então

$$
U^{\mu^{V}}(z)+\frac{V(z)}{2} \geq F^{V}
$$

vale $\mu^{V}$ quase em toda parte em $\mathbb{C}$, onde

$$
F^{V} \equiv E^{V}-\frac{1}{2} \int V(z) d \mu^{V}(z) \text { e } E^{V}=I^{V}\left(\mu^{V}\right)
$$

Prova. Analogamente a prova do Teorema anterior, para $\mu \in \mathcal{M}(\mathbb{C})$, definimos

$$
\mu_{t} \equiv t \mu+(1-t) \mu^{V}=\mu^{V}+t\left(\mu-\mu^{V}\right) \equiv \mu^{V}+t \mu_{s}, t \in[0,1]
$$

Como

$$
I^{V}\left(\mu_{t}\right) \geq I^{V}\left(\mu_{0}\right)=I^{V}\left(\mu^{V}\right)
$$


é satisfeita para todo $t \in[0,1]$, temos, por (B.35)

$$
\begin{aligned}
0 & \leq\left.\frac{1}{2} \frac{d}{d t} I^{V}\left(\mu_{t}\right)\right|_{t=0} \\
& =\int\left(\frac{V(z)}{2}+\int \log |z-w|^{-1} d \mu^{V}(w)\right) d \mu_{s}(z) \\
& =\int\left(U^{\mu^{V}}(z)+\frac{V(z)}{2}\right) d\left(\mu-\mu^{V}\right)(z) \\
& =\int\left(U^{\mu^{V}}(z)+\frac{V(z)}{2}\right) d \mu-\int\left(U^{\mu^{V}}(z)+\frac{V(z)}{2}\right) d \mu^{V}(z) \\
& =\int\left(\frac{1}{2} \int \frac{V(z)}{2}+U^{\mu^{V}}(z)\right) d \mu-E^{V}+\frac{1}{2} \int V(z) d \mu^{V}(z) \\
& =\int\left(\frac{V(z)}{2}+U^{\mu^{V}}(z)\right) d \mu-F^{V}
\end{aligned}
$$

como queríamos.

\section{B.5 Prova do item e}

Teorema B.14 Seja $V$ como no Teorema B.1 e $U^{\mu^{V}}$ o potencial logarítmico associado à medida de equilíbrio $\mu^{V}$. Para quase todo $z \in \mathbb{S}^{V}$

$$
U^{\mu^{V}}(z)+\frac{V(z)}{2}=F^{V} .
$$

Prova. Definimos a função

$$
G^{V}(z) \equiv U^{\mu^{V}}(z)+\frac{V(z)}{2}
$$

o conjunto

$$
\mathbb{A} \equiv\left\{z \in \mathbb{S}^{V}: G^{V}(z)<F^{V}\right\}
$$

e a medida de probabilidade

$$
\mu=\frac{\chi_{\mathbb{A}}}{\mu^{V}(\mathbb{A})} \mu^{V}
$$

onde $\chi_{\mathbb{A}}$ é a função indicadora do conjunto $\mathbb{A}$. Aplicando o Teorema anterior à $\mu$ temos

$$
F^{V} \leq \int G^{V}(z) d \mu(z)=\int G^{V}(z) \frac{\chi_{\mathbb{A}}(z)}{\mu^{V}(\mathbb{A})} \mu^{V}(z)<F^{V}
$$

e, portanto, $\mu(\mathbb{A})=0$, o que prova este Teorema. 


\section{Apêndice C}

\section{Prova dos Teoremas citados no Capítulo 2}

Neste Apêndice descreveremos um método para verificar se uma dada medida de probabilidade é a medida de equilíbrio associada a um potencial $V$ num domínio $\Sigma \subset \mathbb{C}$.

Definição C.1 Denotaremos o produto de convolução entre duas funções $f$ e $g$ por

$$
f * g(z) \equiv \int_{\mathbb{C}} f(z-w) g(w) d^{2} w .
$$

Analogamente, a convolução de uma função $f$ com uma medida de borel com suporte compacto $\mu$ é dada por

$$
f * \mu(z) \equiv \int_{\mathbb{C}} f(z-w) d \mu(w)
$$

Observação C.2 Notemos que com esta notação, o potencial logarítmico $U^{\mu}$ (2.3) pode ser escrito como

$$
U^{\mu}=-\log |\cdot| * \mu .
$$

No Teorema a seguir, as funções convoluídas são sempre limitadas por um lado, integráveis em subconjuntos compactos de $\mathbb{C}$ e, no máximo, uma delas não tem suporte compacto. Estas propriedades implicam, através do Teorema de Fubini, que a convolução é comutativa e associativa, mesmo quando um dos fatores em uma convolução múltipla for uma medida de Borel com suporte compacto.

Prova do Teorema 42. Para $\varepsilon>0$, definimos

$$
\mathbb{D}_{\varepsilon}=\{z \in \mathbb{D}: \operatorname{dist}(z, \partial \mathbb{D})>\varepsilon\}, \operatorname{dist}(z, \partial \mathbb{D}) \equiv \min _{w \in \partial \mathbb{D}}|z-w|
$$




$$
\begin{gathered}
\chi_{\varepsilon}(z) \equiv\left\{\begin{array}{l}
1 \text { se }|z| \leq \varepsilon \\
0 \text { se }|z|>\varepsilon
\end{array}\right. \\
\log _{\varepsilon}(z) \equiv \min \left(\log \frac{1}{|z|}, \log \frac{1}{\varepsilon}\right)=\left\{\begin{array}{l}
\log \frac{1}{|z|} \text { se }|z| \geq \varepsilon \\
\log \frac{1}{\varepsilon} \text { se }|z| \leq \varepsilon
\end{array}\right.
\end{gathered}
$$

e definimos $\mu_{\varepsilon}$ (e analogamente $\left.\nu_{\varepsilon}\right)$ por

$$
\mu_{\varepsilon}(\mathbb{B})=\int \mu(\mathbb{B}-w) \chi_{\varepsilon}(w) d^{2} w
$$

para todo conjunto de Borel $\mathbb{B}$. Notemos, pela Observação anterior, que

$$
f * \mu_{\varepsilon}=f *\left(\mu * \chi_{\varepsilon}\right)=\left(f * \chi_{\varepsilon}\right) * \mu=(f * \mu) * \chi_{\varepsilon} .
$$

Assim, por (C.3) e (C.1) obtemos

$$
U^{\mu_{\varepsilon}}(z)=U^{\mu} * \chi_{\varepsilon}(z)=\left(U^{\nu}(z)+u(z)\right) * \chi_{\varepsilon}(z)=U^{\nu_{\varepsilon}}(z)+u * \chi_{\varepsilon}(z)
$$

e, portanto,

$$
U^{\mu_{\varepsilon}-\nu_{\varepsilon}}(z)=u * \chi_{\varepsilon}(z)
$$

para todo $z \in \mathbb{D}_{\varepsilon}$ (notemos que $u * \chi_{\varepsilon}$ é harmônica em $\mathbb{D}_{\varepsilon}$ ). Como $\log |\cdot| * \chi_{\varepsilon}$ é contínua em $\mathbb{C}$, o mesmo ocorre para

$$
U^{\mu_{\varepsilon}}=(-\log |\cdot| * \mu) * \chi_{\varepsilon}=\left(-\log |\cdot| * \chi_{\varepsilon}\right) * \mu \text { e } U^{\nu_{\varepsilon}}=\left(-\log |\cdot| * \chi_{\varepsilon}\right) * \nu
$$

Integrando a igualdade (C.4) sobre a circunferência

$$
\{\xi \in \mathbb{C}:|z-\xi|=\eta\}, \eta \leq \varepsilon,
$$

usando o Lema B.9 e a notação (C.2), obtemos

$$
\frac{1}{2 \pi} \int_{-\pi}^{\pi} \log \frac{1}{\left|z-\eta e^{i \varphi}-t\right|} d \varphi=\log _{\eta}|z-t|
$$

Assim, pela expressão anterior e pelo Teorema de Fubini, o lado esquerdo de (C.4) pode ser escrito como

$$
\begin{aligned}
\frac{1}{2 \pi} \int_{-\pi}^{\pi} U^{\mu_{\varepsilon}-\nu_{\varepsilon}}\left(z+\eta e^{i \varphi}\right) d \varphi & =\frac{1}{2 \pi} \int_{-\pi}^{\pi} \int \log \left|z+\eta e^{i \varphi}-w\right|^{-1} d\left(\mu_{\varepsilon}-\nu_{\varepsilon}\right)(w) d \varphi \\
& =\int \log _{\eta}|z-t| d\left(\mu_{\varepsilon}-\nu_{\varepsilon}\right)(w) \\
& =\log _{\eta}|\cdot| *\left(\mu_{\varepsilon}-\nu_{\varepsilon}\right)(z)
\end{aligned}
$$


e o lado direito de (C.4), pela propriedade do valor médio para funções harmônicas (Observação B.8), é dado por

$$
\begin{aligned}
\frac{1}{2 \pi} \int_{-\pi}^{\pi} U^{\mu_{\varepsilon}-\nu_{\varepsilon}}\left(z+\eta e^{i \varphi}\right) d \varphi & =\frac{1}{2 \pi} \int_{-\pi}^{\pi} u * \chi_{\varepsilon}\left(z+\eta e^{i \varphi}\right) d \varphi \\
& =u * \chi_{\varepsilon}(z)=U^{\mu_{\varepsilon}-\nu_{\varepsilon}}(z) \\
& =\log _{0}|\cdot| *\left(\mu_{\varepsilon}-\nu_{\varepsilon}\right)(z)
\end{aligned}
$$

para $z \in \mathbb{D}_{2 \varepsilon}$, lembrando que $\eta \leq \varepsilon$ (notamos que (C.7) é bem definida pela continuidade de (C.5) e que $\left.\log _{0}|\cdot|=-\log |\cdot|\right)$.

Subtraindo (C.6) de (C.7) obtemos, para todo $z \in \mathbb{D}_{2 \varepsilon}$ e $0<\eta \leq \varepsilon$

$$
\left(\log _{\eta}|\cdot|-\log _{0}|\cdot|\right) *\left(\mu_{\varepsilon}-\nu_{\varepsilon}\right)(z)=0
$$

Aplicando este procedimento com $\eta$ e $\eta_{1} \leq \varepsilon$ obtemos

$$
\left(\log _{\eta}|\cdot|-\log _{\eta_{1}}|\cdot|\right) *\left(\mu_{\varepsilon}-\nu_{\varepsilon}\right)(z)=0
$$

Finalmente, dividindo esta equação por $\log \frac{\eta_{1}}{\eta}$ e fazendo $\eta_{1} \nearrow \eta$, temos

$$
\begin{aligned}
0 & =\lim _{\eta_{1} \nearrow \eta}\left(\frac{\log _{\eta}|\cdot|-\log _{\eta_{1}}|\cdot|}{\log \frac{\eta_{1}}{\eta}}\right) *\left(\mu_{\varepsilon}-\nu_{\varepsilon}\right)(z) \\
& =\chi_{\eta} *\left(\mu_{\varepsilon}-\nu_{\varepsilon}\right)(z)
\end{aligned}
$$

pelo Teorema da convergência dominada, pois $0 \leq \chi_{\eta} \leq 1$ para todo $0<\eta_{1}<\eta$.

Agora tomemos $h$ uma função contínua que se anula fora de um subconjunto compacto de $\mathbb{D}$. Então, como $\mathbb{D}$ é aberto, existe um $\varepsilon_{0}$ tal que $h$ se anula fora de um subconjunto compacto de $\mathbb{D}_{3 \varepsilon_{0}}$. Escolhendo $0<\eta \leq \varepsilon \leq \varepsilon_{0}$ arbitrário, multiplicando (C.8) por $h$ e integrando o resultado com respeito à medida de Lebesgue no plano, obtemos

$$
\begin{aligned}
0 & =\int h(z) \chi_{\eta} *\left(\mu_{\varepsilon}-\nu_{\varepsilon}\right)(z) d^{2} z \\
& =\int h(z) \int \chi_{\eta}(z-w) d\left(\mu_{\varepsilon}-\nu_{\varepsilon}\right)(w) d^{2} z \\
& =\iint h(w-z) \chi_{\eta}(-z) d^{2} z d\left(\mu_{\varepsilon}-\nu_{\varepsilon}\right)(w) \\
& =\int h * \chi_{\eta} d\left(\mu_{\varepsilon}-\nu_{\varepsilon}\right)
\end{aligned}
$$


onde na penúltima passagem usamos o Teorema de Fubini e na última usamos $\chi_{\eta}(z)=$ $\chi_{\eta}(-z)$. Como

$$
\lim _{\eta \rightarrow 0} \frac{h * \chi_{\eta}}{\pi \eta^{2}}=h
$$

uniformemente, podemos concluir de (C.9) que

$$
0=\lim _{\eta \rightarrow 0} \int \frac{h * \chi_{\eta}}{\pi \eta^{2}} d\left(\mu_{\varepsilon}-\nu_{\varepsilon}\right)=\int h d\left(\mu_{\varepsilon}-\nu_{\varepsilon}\right)
$$

e aplicando o procedimento anterior para $\mu_{\varepsilon}-\nu_{\varepsilon}=\chi_{\varepsilon} *(\mu-\nu)$ obtemos

$$
0=\lim _{\eta \rightarrow 0} \int \frac{h * \chi_{\varepsilon}}{\pi \varepsilon^{2}} d(\mu-\nu)=\int h d(\mu-\nu)
$$

o que prova o Teorema desde que isto é verdade para toda função $h$ com suporte compacto em $\mathbb{D}$.

Lema C.3 (Identidade de Green) Sejam $\mathbb{D}$ um aberto limitado com fronteira $\partial \mathbb{D}$ continuamente diferenciável, $u$ e $v$ funções com segundas derivadas parciais continuas em $\mathbb{D}$ e limitadas em $\overline{\mathbb{D}}$. Então

$$
\int_{\mathbb{D}}(v \Delta u-u \Delta v) d^{2} z=-\int_{\partial \mathbb{D}}\left(v \frac{\partial u}{\partial n}-u \frac{\partial v}{\partial n}\right) d z
$$

onde $\Delta$ é o operador laplaciano, $\frac{\partial}{\partial n}$ denota derivação na direção da normal interna de $\mathbb{D}$ e dz indica integração com respeito ao comprimento de arco de $\partial \mathbb{D}$.

Para uma demonstração deste Teorema veja [39] página 280.

Prova do Teorema 43. Seja $\mathbb{D}_{0} \subset \mathbb{D}$ um subconjunto simplesmente conexo de $\mathbb{D}$ com fronteira $\partial \mathbb{D}$ continuamente diferenciável e fecho $\overline{\mathbb{D}_{0}} \subset \mathbb{D}$. Este Teorema segue do Teorema anterior se provarmos que a função

$$
\frac{1}{2 \pi} \int_{\mathbb{D}_{0}} \log |z-w|^{-1} \Delta U^{\mu}(w) d^{2} w+U^{\mu}(w)
$$

é harmônica em $\mathbb{D}_{0}$ com $\mathbb{D}_{0}$ arbitrário.

Para $z_{0} \in \mathbb{D}_{0}$ e $r>0$ suficientemente pequeno, seja

$$
\mathbb{D}_{r}=\mathbb{D}_{0} \backslash \overline{\mathbb{B}_{r}\left(z_{0}\right)}
$$

onde

$$
\mathbb{B}_{r}\left(z_{0}\right)=\left\{z \in \mathbb{C}:\left|z-z_{0}\right|<r\right\} .
$$

Aplicando a identidade de Green para

$$
u \equiv U^{\mu} \text { e } v(z) \equiv \frac{1}{2 \pi} \log \left|z_{0}-z\right|^{-1},
$$


o lado esquerdo de (C.10) fica

$$
\int_{\mathbb{D}_{r}} v \Delta u d^{2} w=\int_{\mathbb{D}_{0}} \log |z-w|^{-1} \Delta U^{\mu}(w) d^{2} w-O\left(r^{2} \log r\right)
$$

pois $v$ é harmônica em $\mathbb{D}_{r}$. Notemos que a expressão anterior coincide com a primeira parcela de (C.11) exceto pela integral sobre $\mathbb{B}_{r}\left(z_{0}\right)$ que é ordem $O\left(r^{2} \log r\right)$. O lado esquerdo de (C.10) fica

$$
-\int_{\partial \mathbb{D}_{0}}\left(v \frac{\partial u}{\partial n}-u \frac{\partial v}{\partial n}\right) d z-\int_{\partial \mathbb{B}_{r}\left(z_{0}\right)}\left(v \frac{\partial u}{\partial n}-u \frac{\partial v}{\partial n}\right) d z
$$

pois $\partial \mathbb{D}_{r}=\partial \mathbb{B}_{r}\left(z_{0}\right) \cup \partial \mathbb{D}_{r}$. Como para $z \neq z_{0}$ ambas as funções $v$ e $\frac{\partial v}{\partial n}$ são harmônicas em $\mathbb{D}_{0}$ como função de $z_{0}$, a contribuição das integrais sobre $\partial \mathbb{D}_{0}$ é uma função harmônica em $\mathbb{D}_{0}$. A primeira integral sobre $\partial \mathbb{B}_{r}\left(z_{0}\right)$ é

$$
-\int_{\partial \mathbb{B}_{r}\left(z_{0}\right)} v \frac{\partial u}{\partial n} d z=O\left(r \log \frac{1}{r}\right)
$$

e a segunda

$$
\begin{aligned}
\int_{\partial \mathbb{B}_{r}\left(z_{0}\right)} u \frac{\partial v}{\partial n} d z & =-\frac{1}{2 \pi} \int_{\partial \mathbb{B}_{r}\left(z_{0}\right)} U^{\mu}(z) \frac{1}{\left|z_{0}-z\right|} d z \\
& =-\frac{1}{2 \pi} \int_{\partial \mathbb{B}_{r}\left(z_{0}\right)} \frac{U^{\mu}(z)}{r} d z \\
& =-U^{\mu}\left(z_{0}\right)+o(1)
\end{aligned}
$$

quando $r \rightarrow \infty$. Isto prova que (C.11) é uma função harmônica em $\mathbb{D}_{0}$ e, conseqüentemente, o Teorema fica provado.

Teorema C.4 (Princípio da Dominação) Sejam $\mu$ e $\nu$ duas medidas de Borel positivas e finitas com suporte compacto em $\mathbb{C}, \nu(\mathbb{C}) \leq \mu(\mathbb{C})$ e que $\mu$ tenha potencial logarítmico $U^{\mu}$ finito. Se, para alguma constante $c$, a desigualdade

$$
U^{\mu}(z) \leq U^{\nu}(z)+c
$$

vale $\mu$-quase toda parte, então ela vale para todo $z \in \mathbb{C}$. Admitiremos o Princípio da Dominação sem demonstração. Veja Capítulo II Teorema 3.2 de [24] para uma demons$\operatorname{tração.~}$

Por comodidade enunciaremos novamente o Teorema 45. 
Sejam $V, \Sigma, \Sigma_{0}, F^{V}, \mu^{V}$ e $\mathbb{S}^{\mu^{V}}$ como no Teorema 40. Então, para toda medida de probabilidade $\nu$ com suporte $\mathbb{S}^{\nu}$ contido em $\Sigma$.

$$
\inf _{z \in \Sigma_{0}}\left(U^{\nu}(z)+\frac{V(z)}{2}\right) \leq F^{V}
$$

e

$$
\sup _{z \in \mathbb{S}^{\nu}}\left(U^{\nu}(z)+\frac{V(z)}{2}\right) \geq F^{V} .
$$

Se a igualdade valer em (C.13) e (C.14), então $\nu=\mu^{V}$.

Prova do Teorema 45. Pelo Teorema 40 temos para a medida de equilíbrio

$$
\inf _{z \in \Sigma_{0}}\left(U^{\mu^{V}}(z)+\frac{V(z)}{2}\right)=F^{V}
$$

e

$$
\sup _{z \in \mathbb{S}^{\sigma}}\left(U^{\mu^{V}}(z)+\frac{V(z)}{2}\right)=F^{V} .
$$

Agora, consideramos (C.13) e suponhamos que para algum $L_{1} \in \mathbb{R}$

$$
\inf _{z \in \Sigma_{0}}\left(U^{\nu}(z)+\frac{V(z)}{2}\right)=L_{1}
$$

e usando (C.16) obtemos, para todo $z \in \mathbb{S}^{\sigma}$,

$$
U^{\nu}(z) \geq L_{1}-\frac{V(z)}{2} \geq L_{1}-F^{V}+U^{\mu^{V}}(z)
$$

Como $\mu^{V}$ tem energia logarítmica finita (pois é uma medida de equilíbrio e, portanto, com suporte compacto), (C.17) vale para todo $z \in \Sigma_{0}$. Usando o Princípio da dominação (C.4), (C.17) vale para todo $z \in \mathbb{C}$ e, tomando $z \rightarrow \infty$ obtemos

$$
F^{V} \geq L_{1}
$$

pois $U^{\nu}$ e $U^{\mu^{V}}$ possuem o mesmo comportamento para $z \in \mathbb{C} \backslash\left(\mathbb{S}^{\nu} \cup \mathbb{S}^{\mu^{V}}\right)$ e, portanto, (C.13) fica provado. Para provarmos (C.14), suponhamos que para todo $z \in \mathbb{S}^{\nu}$

$$
U^{\nu}(z)+\frac{V(z)}{2} \leq L_{2} \leq F^{V}
$$

Assim $L_{2}$ é finito e como $V$ é limitado inferiormente concluímos de (C.18) que $\nu$ tem energia logarítmica finita. Assim, subtraindo as desigualdades (C.18) e (C.15) temos

$$
U^{\mu^{V}}(z)-U^{\nu}(z) \leq F^{V}-L_{2}
$$

e novamente fazendo $z \rightarrow \infty$ obtemos

$$
F^{V} \leq L_{2}
$$


e com isso provamos (C.14) por absurdo.

Agora, tomando $L_{1}=L_{2}=F^{V}$, concluímos que os potenciais logarítmicos de $\nu$ e $\mu^{V}$ coincidem em toda a parte e, portanto, $\nu=\mu^{V}$ pelo Lema B.11.

Prova do Teorema 46. Das hipóteses deste Teorema temos

$$
\inf _{z \in \Sigma_{0}}\left(U^{\nu}(z)+\frac{V(z)}{2}\right)=F
$$

$\mathrm{e}$

$$
\sup _{z \in \mathbb{S}^{\sigma}}\left(U^{\nu}(z)+\frac{V(z)}{2}\right)=F
$$

de onde se conclui que $F^{V} \leq F \leq F^{V}$ e, portanto, $F=F^{V}$ e $\nu=\mu^{V}$ pelo Teorema anterior. A segunda parte segue do Princípio da Dominação (Teorema C.4). De fato, por um lado

$$
U^{\nu}(z)-F \leq U^{\mu^{V}}(z)-F^{V}, z \in \mathbb{C}
$$

e por outro lado a desigualdade oposta. Logo fazendo $z \rightarrow \infty$ obtemos

$$
F=F^{V},
$$

que implica

$$
U^{\nu}(z) \equiv U^{\mu^{V}}(z)
$$

e $\nu=\mu^{V}$ pelo Lema B.11. 


\section{Apêndice D}

\section{Cálculo de algumas medidas de equilíbrio}

Aplicando os Teoremas da Subseção 2.1, obteremos explicitamente as medidas de equilíbrio citadas no decorrer da tese.

\section{D.1 Potenciais Radialmente simétricos}

Prova do Teorema 52. Definimos

$$
d \nu(z)=\frac{1}{4 \pi}\left(r V_{C}^{\prime}(r)\right)^{\prime} \chi_{\left[r_{0}, R_{0}\right]}(r) d r d \theta
$$

com $r_{0}$ e $R_{0}$ definidos no enunciado do Teorema. Notemos que $\nu$ é uma medida de probabilidade:

$$
\int d \nu(z)=\frac{1}{4 \pi} \int_{r_{0}}^{R_{0}} \int_{-\pi}^{\pi}\left(r V_{C}^{\prime}(r)\right)^{\prime} d \theta d r=\frac{1}{2}\left(R_{0} V_{C}^{\prime}\left(R_{0}\right)-r_{0} V_{C}^{\prime}\left(r_{0}\right)\right)=1 .
$$

O potencial logarítmico associado a esta medida é

$$
U^{\nu}(z)=\int \log |z-w|^{-1} d \nu(w)=\frac{1}{4 \pi} \int_{r_{0}}^{R_{0}} \int_{-\pi}^{\pi} \log \left|z-s e^{i \theta}\right|^{-1}\left(s V_{C}^{\prime}(s)\right)^{\prime} d s d \theta
$$

Pelo Lema B.9, para todo $s>0$

$$
\frac{1}{2 \pi} \int_{-\pi}^{\pi} \log \frac{1}{\mid z-s e^{i \theta \mid}} d \theta=\left\{\begin{array}{c}
\log \frac{1}{s} \text { se }|z| \leq s \\
\log \frac{1}{|z|} \text { se }|z| \geq s
\end{array}\right.
$$


$\mathrm{e}$, portanto, para $|z|=r<r_{0}$ temos

$$
\begin{aligned}
U^{\nu}(z) & =\frac{1}{2} \int_{r_{0}}^{R_{0}}\left(\log \frac{1}{s}\right)\left(s V_{C}^{\prime}(s)\right)^{\prime} d s \\
& =-\frac{1}{2}\left(\left.(\log s)\left(s V_{C}^{\prime}(s)\right)\right|_{r_{0}} ^{R_{0}}-\int_{r_{0}}^{R_{0}} V_{C}^{\prime}(s) d s\right) \\
& =-\frac{1}{2}\left(2 \log R_{0}-\left(V_{C}\left(R_{0}\right)-V_{C}\left(r_{0}\right)\right)\right) \\
& =F^{V}-\frac{V_{C}\left(r_{0}\right)}{2}
\end{aligned}
$$

onde

$$
F^{V}=\frac{V_{C}\left(R_{0}\right)}{2}-\log R_{0}
$$

Para $|z|=r, r_{0} \leq r \leq R_{0}$, temos

$$
\begin{aligned}
U^{\nu}(z) & =\frac{1}{2}\left(\int_{r_{0}}^{r}\left(\log \frac{1}{r}\right)\left(s V_{C}^{\prime}(s)\right)^{\prime} d s+\int_{r}^{R_{0}}\left(\log \frac{1}{s}\right)\left(s V_{C}^{\prime}(s)\right)^{\prime} d s\right) \\
& =-\frac{1}{2}\left(\left.(\log r)\left(s V_{C}^{\prime}(s)\right)\right|_{r_{0}} ^{r}+\left.(\log s)\left(s V_{C}^{\prime}(s)\right)\right|_{r} ^{R_{0}}-\int_{r}^{R_{0}} V_{C}^{\prime}(s) d s\right) \\
& =-\frac{1}{2}\left(2 \log R_{0}-V_{C}\left(R_{0}\right)+V_{C}(r)\right)=F^{V}-\frac{V_{C}(r)}{2}
\end{aligned}
$$

e finalmente para $|z|=r \geq R_{0}$

$$
\begin{aligned}
U^{\nu}(z) & =\frac{1}{2} \int_{r_{0}}^{R_{0}} \log \frac{1}{r}\left(s V_{C}^{\prime}(s)\right)^{\prime} d s \\
& =-\frac{1}{2}\left(\left.\log r\left(s V_{C}^{\prime}(s)\right)\right|_{r_{0}} ^{R_{0}}\right)=-\log r .
\end{aligned}
$$

Logo, de (D.1), (D.2) e (D.3) obtemos

$$
U^{\nu}(z)=\left\{\begin{array}{l}
F^{V}-\frac{V\left(r_{0}\right)}{2}, \text { se }|z| \leq r_{0} \\
F^{V}-\frac{V(z)}{2} \text { se } r_{0} \leq|z| \leq R_{0} \\
-\log |z|, \text { se }|z| \geq R_{0}
\end{array}\right.
$$

e, portanto, concluímos que $\nu=\mu^{V}$ é a medida de equilíbrio associada à $V$ usando o Teorema 46. 
Corolário D.1 Seja $V_{N}: \Sigma=\mathbb{C} \rightarrow \mathbb{R}$ dado por

$$
V_{\alpha}(z)=|z|^{\alpha}, \alpha>0
$$

Então, a medida de equilíbrio $\mu^{V_{\alpha}}$ associada à $V_{N}$ é tal que sua densidade é dada pela distribuição

$$
d \mu^{V_{\alpha}}(z)=\frac{\alpha^{2}}{4 \pi}|z|^{\alpha-1} \chi_{\left[r_{0}, R_{0}\right]}(r) d r d \theta
$$

Prova. Segue diretamente do Teorema anterior, com $r_{0}=0$ e $R_{0}=\left(\frac{2}{\alpha}\right)^{\frac{1}{\alpha}}$ e $F^{V_{N}}=$ $\frac{1}{\alpha}\left(1-\log \left(\frac{2}{\alpha}\right)\right)$.

\section{D.2 Ensemble de Dyson no formalismo da Medida de Equilíbrio}

Prova do Teorema 55. o potencial logarítmico para $\mu^{V_{D}}$ é

$$
U^{\mu^{V_{D}}}(z)=\int \log |z-w|^{-1} d \mu^{V_{D}}(w)=\frac{1}{2 \pi} \int_{-\pi}^{\pi} \log \left|z-e^{i \theta}\right|^{-1} d \theta
$$

Mas pelo Lema (B.9), para todo $r>0$

$$
\frac{1}{2 \pi} \int_{-\pi}^{\pi} \log \frac{1}{\mid z-r e^{i \theta \mid}} d \theta=\left\{\begin{array}{l}
\log \frac{1}{r} \text { se }|z| \leq r \\
\log \frac{1}{|z|} \text { se }|z| \geq r
\end{array} .\right.
$$

Logo

$$
U^{\mu^{V_{D}}}(z)=\frac{1}{2 \pi} \int_{-\pi}^{\pi} \log \left|z-e^{i \theta}\right|^{-1} d \theta=\left\{\begin{array}{c}
0 \text { se }|z| \leq 1 \\
\log \frac{1}{|z|} \text { se }|z| \geq 1
\end{array}\right.
$$

e, portanto, concluímos que $\mu^{V_{D}}$ é a medida de equilíbrio associada (2.14) pelo Teorema (46).

Observação D.2 Notamos que o Teorema anterior segue analogamente para a escolha

$$
\Sigma=\Sigma_{D}=\{z \in \mathbb{C}:|z|=1\} \text { e } V_{D} \equiv 0 \text { er } \Sigma_{D}
$$




\section{D.3 Potenciais do tipo $V_{H}(x)=\gamma_{\alpha}|x|^{\alpha}$}

Lema D.3 Sejam

$$
\Sigma=[-u, u] \subset \mathbb{R} \text { e } V_{A S} \equiv 0 \text { em } \Sigma .
$$

Então a medida de equilíbrio $\mu_{A S}$ associada à (D.5) é tal que

$$
\mu_{A S}^{\prime}(x)=\frac{1}{\pi} \frac{1}{\sqrt{u^{2}-x^{2}}} \chi_{\Sigma}(x)
$$

onde $\chi_{\Sigma}$ é a função indicadora do conjunto $\Sigma$.

Prova. O potencial logarítmico para $\mu^{V_{D}}$ é

$$
U^{\mu_{A S}}(z)=\int \log |z-w|^{-1} d \mu_{A S}(w)=\frac{1}{\pi} \int_{-u}^{u} \log |z-t|^{-1} \frac{d t}{\sqrt{u^{2}-t^{2}}}
$$

e fazendo a mudança de variável

$$
t=u \cos \theta
$$

temos

$$
\begin{aligned}
U^{\mu_{A S}}(z) & =\frac{1}{\pi} \int_{\pi}^{0} \log |z-u \cos \theta|^{-1} \frac{-u \operatorname{sen} \theta d \theta}{\sqrt{u^{2}-u^{2} \cos ^{2} \theta}} \\
& =\frac{1}{2 \pi} \int_{-\pi}^{\pi} \log \left|\frac{z}{u}-\cos \theta\right|^{-1} d \theta-\log u
\end{aligned}
$$

Agora, consideramos a transformação conforme de Joukowski

$$
z=\frac{u}{2}\left(w+w^{-1}\right)
$$

que mapeia o conjunto

$$
\{w \in \mathbb{C}:|w|>1\} \text { em }\{z \in \mathbb{C}: z \notin[-u, u]\}
$$

e o círculo

$$
\{w \in \mathbb{C}:|w|=1\} \text { em }\{z \in \mathbb{C}: z \in[-u, u]\}
$$

coberto duas vezes. A transformação inversa a (D.7) é

$$
w=\frac{z+\sqrt{z^{2}-u^{2}}}{u}
$$


com $\sqrt{z^{2}-u^{2}}$ denotando o ramo que se comporta como $z$ nas proximidades do infinito. Definindo $t=e^{i \theta}$ podemos escrever, usando (D.7),

$$
\begin{aligned}
\left|\frac{z}{u}-\cos \theta\right| & =\left|\frac{1}{2}\left(w+w^{-1}\right)-\frac{1}{2}\left(t+t^{-1}\right)\right| \\
& =\frac{1}{2}\left|-t\left(w+w^{-1}+t+t^{-1}\right)\right| \\
& =\frac{1}{2}\left|-t\left(w+w^{-1}\right)+1+t^{2}\right| \\
& =\frac{1}{2}|w-t|\left|w^{-1}-t\right|
\end{aligned}
$$

Assim

$$
\begin{aligned}
U^{\mu_{A S}}(z) & =\frac{1}{2 \pi} \int_{-\pi}^{\pi} \log \frac{2}{|w-t|\left|w^{-1}-t\right|} d \theta-\log u \\
& =\log 2+U^{\mu^{V_{D}}}(w)+U^{\mu^{V_{D}}}\left(w^{-1}\right)-\log u
\end{aligned}
$$

onde $U^{\mu^{V_{D}}}$ é dado por (D.4). Logo, como $|w| \geq 1$

$$
U^{\mu_{A S}}(z)=\log \frac{2}{u}+\log \frac{1}{|w|}+0=\log \frac{2}{u}-\log \left|\frac{z+\sqrt{z^{2}-u^{2}}}{u}\right|
$$

por (D.8). Em particular para $z \in \Sigma=[-u, u]$ temos

$$
U^{\mu_{A S}}(z)=\log \frac{2}{u}-\log \left|\frac{z+i \sqrt{u^{2}-z^{2}}}{u}\right|=\log \frac{2}{u}-\log 1=\log \frac{2}{u}
$$

e, portanto, concluímos que $\mu_{A S}$ é a medida de equilíbrio associada (D.5) pelo Teorema $(46)$.

Teorema D.4 Seja $V_{H}: \Sigma=\mathbb{R} \rightarrow \mathbb{R}$ dado por

$$
V_{H}(x)=\gamma_{\alpha}|x|^{\alpha}, \alpha>0 \text { e } \gamma_{\alpha}=\frac{\Gamma\left(\frac{\alpha}{2}\right) \Gamma\left(\frac{1}{2}\right)}{\Gamma\left(\frac{\alpha+1}{2}\right)} .
$$

Então, a medida de equilíbrio $\mu_{H}$ associada à $V_{H}$ é tal que sua densidade é dada pela distribuição de Ullman

$$
\mu_{H}^{\prime}(x)=\frac{\alpha}{\pi} \int_{|x|}^{1} \frac{u^{\alpha-1}}{\sqrt{u^{2}-x^{2}}} d u \chi_{\mathbb{S}^{\mu} H}(x) \text { onde } \mathbb{S}^{\mu_{H}}=[-1,1]
$$


Prova. Consideramos a distribuição de Ullman

$$
\nu_{\alpha}^{\prime}(x)=\frac{\alpha}{\pi} \int_{|x|}^{1} \frac{u^{\alpha-1}}{\sqrt{u^{2}-x^{2}}} d u \chi_{[-1,1]}(x)
$$

associado a uma medida de probabilidade $\nu_{\alpha}$. Então o potencial logarítmico associado a esta medida é

$$
U^{\nu_{\alpha}}(x)=\int \log |x-t|^{-1} d \nu_{\alpha}(t)=\frac{\alpha}{\pi} \int_{-1}^{1} \log |x-t|^{-1} \int_{|t|}^{1} \frac{u^{\alpha-1}}{\sqrt{u^{2}-t^{2}}} d u d t .
$$

Fazendo a mudança de ordem das integrais (mudança lícita pelo Teorema de Fubini)

$$
U^{\nu_{\alpha}}(x)=\int_{0}^{1} \alpha u^{\alpha-1} \frac{1}{\pi} \int_{-u}^{u} \frac{\log |x-t|^{-1}}{\sqrt{u^{2}-t^{2}}} d t d u .
$$

Por (D.6) e (D.9) notamos que

$$
\frac{1}{\pi} \int_{-u}^{u} \frac{\log |x-t|^{-1}}{\sqrt{u^{2}-t^{2}}} d t=U^{\mu_{A S}}(x)=\left\{\begin{array}{l}
\log \frac{2}{u} \text { se }|x| \leq u \\
\log 2-\log \left|x+\sqrt{x^{2}-u^{2}}\right| \text { se }|x| \geq u
\end{array} .\right.
$$

Logo, para $|x| \leq 1$ (por simetria podemos assumir $0 \leq x \leq 1$ )

$$
\begin{aligned}
U^{\nu_{\alpha}}(x) & =\int_{0}^{x} \alpha u^{\alpha-1}\left(\log 2-\log \left(x+\sqrt{x^{2}-u^{2}}\right)\right) d u+\int_{x}^{1} \alpha u^{\alpha-1} \log \frac{2}{u} d u \\
& =\log 2-\int_{x}^{1} \alpha u^{\alpha-1} \log u d u-\int_{0}^{x} \alpha u^{\alpha-1} \log \left(x+\sqrt{x^{2}-u^{2}}\right) d u \\
& =\log 2+\frac{1}{\alpha}+x^{\alpha}\left(-\frac{1}{\alpha}+\log x\right)-\int_{0}^{1} \alpha x^{\alpha-1} v^{\alpha-1} \log \left(x\left(1+\sqrt{1-v^{2}}\right)\right) x d v \\
& =\log 2+\frac{1}{\alpha}-x^{\alpha}(\frac{1}{\alpha}+\overbrace{\int_{0}^{1} \alpha v^{\alpha-1} \log \left(1+\sqrt{1-v^{2}}\right) d v}^{I}) .
\end{aligned}
$$

onde na penúltima passagem usamos a mudança de coordenadas

$$
u=x v \text {. }
$$

Integrando por partes a integral $I$ temos

$$
\begin{aligned}
I & =0-\int_{0}^{1} \frac{v^{\alpha-1}}{1+\sqrt{1-v^{2}}} \frac{-v^{2}}{\sqrt{1-v^{2}}} d v \\
& =\int_{0}^{1} \frac{v^{\alpha-1}}{1+\sqrt{1-v^{2}}} \frac{\left(1+\sqrt{1-v^{2}}\right)\left(1-\sqrt{1-v^{2}}\right)}{\sqrt{1-v^{2}}} d v \\
& =\int_{0}^{1} \frac{v^{\alpha-1}\left(1-\sqrt{1-v^{2}}\right)}{\sqrt{1-v^{2}}} d v=\gamma_{\alpha}-\frac{1}{\alpha}
\end{aligned}
$$


onde

$$
\frac{\gamma_{\alpha}}{2}=\int_{0}^{1} \frac{v^{\alpha-1}}{\sqrt{1-v^{2}}} d v=\frac{\Gamma\left(\frac{\alpha}{2}\right) \Gamma\left(\frac{1}{2}\right)}{2 \Gamma\left(\frac{\alpha+1}{2}\right)} .
$$

Assim, de (D.12) e (D.13) concluímos que para $|x| \leq 1$

$$
U^{\nu_{\alpha}}(x)=-\frac{\gamma_{\alpha}|x|^{\alpha}}{2}+\log 2+\frac{1}{\alpha} .
$$

Agora, para $|x| \geq 1$ temos de (D.10) e (D.11) (por simetria podemos assumir $x>1$ )

$$
\begin{aligned}
U^{\nu_{\alpha}}(x) & =\int_{0}^{1} \alpha u^{\alpha-1}\left(\log 2-\log \left(x+\sqrt{x^{2}-u^{2}}\right)\right) d u \\
& =\log 2-\int_{0}^{1} \alpha u^{\alpha-1} \log \left(x+\sqrt{x^{2}-u^{2}}\right) d u
\end{aligned}
$$

Calculando a derivada de $U^{\nu_{\alpha}}$ em relação a $x$ temos

$$
\begin{aligned}
\frac{d}{d x} U^{\nu_{\alpha}}(x) & =-\int_{0}^{1} \alpha u^{\alpha-1} \frac{1+x\left(x^{2}-u^{2}\right)^{-\frac{1}{2}}}{x+\sqrt{x^{2}-u^{2}}} d u=-\int_{0}^{1} \alpha u^{\alpha-1} \frac{1+\frac{x}{\sqrt{x^{2}-u^{2}}}}{x+\sqrt{x^{2}-u^{2}}} d u \\
& =-\int_{0}^{1} \frac{\alpha u^{\alpha-1}}{\sqrt{x^{2}-u^{2}}} d u=-\alpha x^{\alpha-1} \int_{0}^{\frac{1}{x}} \frac{v^{\alpha-1}}{\sqrt{1-v^{2}}} d v \geq-\frac{\alpha \gamma_{\alpha}}{2} x^{\alpha-1}
\end{aligned}
$$

onde a igualdade ocorrendo apenas em $x=1$. Além disso

$$
\begin{aligned}
U^{\nu_{\alpha}}(x) & =\int_{0}^{1} \alpha u^{\alpha-1}\left(\log 2-\log \left(x+\sqrt{x^{2}-u^{2}}\right)\right) d u \\
& =\log 2-\int_{0}^{1} \alpha u^{\alpha-1} \log \left(x+\sqrt{x^{2}-u^{2}}\right) d u \\
& =\log 2-\log \left(x+\sqrt{x^{2}-1}\right)-\int_{0}^{1} \frac{u^{\alpha-1}}{x+\sqrt{x^{2}-u^{2}}} \frac{u^{2}}{\sqrt{x^{2}-u^{2}}} d u \\
& =\log 2-\log \left(x+\sqrt{x^{2}-1}\right)-\int_{0}^{1} \frac{x u^{\alpha-1}}{\sqrt{x^{2}-u^{2}}} d u+\frac{1}{\alpha} \\
& =\log 2+\frac{1}{\alpha}-\log \left(x+\sqrt{x^{2}-1}\right)-x^{\alpha} \int_{0}^{\frac{1}{x}} \frac{v^{\alpha-1}}{\sqrt{1-v^{2}}} d v
\end{aligned}
$$

e, portanto,

$$
\lim _{x \rightarrow 1^{+}} U^{\nu_{\alpha}}(x)=\lim _{x \rightarrow 1^{-}} U^{\nu_{\alpha}}(x)=\log 2+\frac{1}{\alpha}-\frac{\gamma_{\alpha}}{2} .
$$

Assim, temos que $V_{H}(x)$

$$
U^{\nu_{\alpha}}(x)+\frac{V_{H}(x)}{2}=\log 2+\frac{1}{\alpha}
$$


para $x \in \mathbb{S}^{\mu_{H}}=[-1,1] \mathrm{e}$

$$
U^{\nu_{\alpha}}(x)+\frac{V_{H}(x)}{2} \geq \log 2+\frac{1}{\alpha}
$$

para $x \in \mathbb{R}$. Logo este Teorema fica provado aplicando o Teorema (46) e definindo $F^{V}=\log 2+\alpha^{-1}$.

Observação D.5 Se tomarmos um potencial mais geral do tipo

$$
\widetilde{V}_{H}(x)=c|x|^{\alpha}, c>0
$$

basta reescalarmos o resultado anterior:

$$
\widetilde{V}_{H}(x)=c|x|^{\alpha}=\gamma_{\alpha}\left|\frac{x}{s}\right|^{\alpha}=V_{H}\left(\frac{x}{s}\right) \operatorname{com} s=\left(\frac{\gamma_{\alpha}}{c}\right)^{\frac{1}{\alpha}} .
$$

Assim obteremos

$$
\mathbb{S}^{\widetilde{\mu}_{H}}=[-s, s]
$$

e

$$
\widetilde{\mu}_{H}^{\prime}(x)=\frac{1}{s} \mu_{H}^{\prime}\left(\frac{x}{s}\right)=\frac{\alpha}{\pi s} \int_{\left|\frac{x}{s}\right|}^{1} \frac{u^{\alpha-1}}{\sqrt{u^{2}-\left(\frac{x}{s}\right)^{2}}} d u \chi_{\mathbb{S}_{\tilde{\mu}} \tilde{\mu}}(x)
$$

Corolário D.6 (Lei do Semicírculo) Seja $V_{W}: \Sigma=\mathbb{R} \rightarrow \mathbb{R}$ dado por

$$
V_{W}(x)=\frac{x^{2}}{2}
$$

Então, a medida de equilíbrio $\mu_{W}$ associada à $V_{W}$ é tal que sua densidade é dada pela lei do semicírculo

$$
\mu_{W}^{\prime}(x)=\frac{1}{2 \pi} \sqrt{4-x^{2}} \chi_{[-2,2]}(x)
$$

Prova. Consideramos o Teorema anterior e tomemos $\alpha=2$. Assim obtemos

$$
V_{H}(x)=2 x^{2}
$$

pois

$$
\gamma_{2}=\frac{\Gamma\left(\frac{2}{2}\right) \Gamma\left(\frac{1}{2}\right)}{\Gamma\left(\frac{2+1}{2}\right)}=\frac{\Gamma\left(\frac{1}{2}\right)}{\frac{1}{2} \Gamma\left(\frac{1}{2}\right)}=2
$$

e, portanto,

$$
\mu_{H}^{\prime}(x)=\frac{2}{\pi} \sqrt{1-x^{2}} \chi_{[-1,1]}(x)
$$

Logo, como

$$
V_{W}(x)=\frac{x^{2}}{2}
$$


temos da Observação anterior que $c=\frac{1}{2}$ e $s=\left(\frac{2}{\frac{1}{2}}\right)^{\frac{1}{2}}=2$ e, portanto,

$$
\mathbb{S}^{\mu_{W}}=[-2,2]
$$

e

$$
\mu_{W}^{\prime}(x)=\frac{1}{2} \mu_{H}^{\prime}\left(\frac{x}{2}\right)=\frac{1}{\pi} \sqrt{1-\left(\frac{x}{2}\right)^{2}} \chi_{\mathbb{S}^{\mu} W}(x)=\frac{1}{2 \pi} \sqrt{4-x^{2}} \chi_{\mathbb{S}^{\mu} W}(x)
$$

como queríamos. 


\section{Apêndice E}

\section{Ensemble normal de Ginibre-Girko}

Esta seção tem como objetivo dar uma prova completa e detalhada de resultados já conhecidos para este ensemble. Além de ser uma prova que novamente ilustra o método dos polinômios ortogonais, não encontramos na literatura esta prova completa, de forma que alguns dos passos da prova ficam remetidos a outros textos.

O ensemble normal de Ginibre-Girko, cujo potencial associado é

$$
\begin{aligned}
V_{\tau}(z) & =\frac{1}{1-\tau^{2}}\left(|z|^{2}-\tau\left(\frac{z^{2}+\bar{z}^{2}}{2}\right)\right) \\
& =\frac{x^{2}}{1+\tau}+\frac{y^{2}}{1-\tau} .
\end{aligned}
$$

$\operatorname{com} \tau \in] 0,1[$.

Lema E.1 Sejam $\left\{\phi_{j}^{G}\right\}_{j=1}^{N}$ os polinômios ortonormais com respeito ao peso

$$
d \nu^{G}(z)=e^{-N V_{\tau}(z)} d^{2} z
$$

e $V_{\tau}: \mathbb{C} \rightarrow \mathbb{R}$ o potencial dado por (E.1) (notamos que este tipo de potencial satisfaz as hipóteses do Teorema 40). Então

$$
\phi_{j+1}^{G}(z)=\frac{\tau^{\frac{j}{2}} \sqrt{N}}{\sqrt{j ! \pi}\left(1-\tau^{2}\right)^{\frac{1}{4}}} H_{j+1}\left(\sqrt{\frac{N}{\tau}} z\right)
$$

onde $H_{j}$ é o j-ésimo polinômio mônico de Hermite

$$
e^{x \tau^{2}-\frac{\tau^{2}}{2}}=\sum_{j=0}^{\infty} H_{j+1}(x) \frac{\tau^{j}}{j !}
$$

e $H_{j}(z)$ para $z \in \mathbb{C}$ a extensão analítica de $H_{j}(x), x \in \mathbb{R}$. 
Prova. Consideramos a identidade

$$
\int e^{-\frac{1}{2} Z^{*} A Z} \frac{d^{2} z}{\pi}=\frac{2}{\sqrt{(a+d)^{2}-4 c \bar{c}}}
$$

onde $a, c, d, z \in \mathbb{C}$ e

$$
Z=\left(\begin{array}{c}
z \\
\bar{z}
\end{array}\right), A=\left(\begin{array}{ll}
a & c \\
\bar{c} & d
\end{array}\right) \text { e } Z^{*}=\bar{Z}^{t}
$$

Tomando $A$ de modo que

$$
\frac{Z^{*} A Z}{2}=|z|^{2}-\tau\left(\frac{z^{2}+\bar{z}^{2}}{2}\right)
$$

obtemos

$$
A=\left(\begin{array}{cc}
1 & -\tau \\
-\tau & 1
\end{array}\right) \Longrightarrow A^{-1}=\frac{1}{1-\tau^{2}}\left(\begin{array}{cc}
1 & \tau \\
\tau & 1
\end{array}\right)
$$

e, portanto, para esta escolha de $A$ temos

$$
\int e^{-\frac{1}{2} Z^{*} A Z} \frac{d^{2} z}{\pi}=\frac{1}{\sqrt{1-\tau^{2}}} .
$$

Definimos também

$$
X=\left(\begin{array}{l}
x \\
\bar{x}
\end{array}\right), x \in \mathbb{C} \mathrm{e}\langle\cdot\rangle \equiv \frac{\int \cdot e^{-\frac{1}{2} Z^{*} A Z} \frac{d^{2} z}{\pi}}{\int e^{-\frac{1}{2} Z^{*} A Z} \frac{d^{2} z}{\pi}}
$$

Assim

$$
\begin{aligned}
1 & =\frac{\int e^{-\frac{1}{2}\left(Z-A^{-1} X\right)^{*} A\left(Z-A^{-1} X\right) \frac{d^{2} z}{\pi}}}{\int e^{-\frac{1}{2} Z^{*} A Z \frac{d^{2} z}{\pi}}} \\
& =\left\langle e^{\frac{1}{2}\left(X^{*} Z+X Z^{*}\right)-\frac{1}{2}\left(X^{*} A^{-1} X\right)}\right\rangle \\
& =\left\langle e^{\bar{x} z+x \bar{z}-\frac{1}{1-\tau^{2}}\left(x \bar{x}+\frac{\tau}{2}\left(x^{2}+\bar{x}^{2}\right)\right)}\right\rangle .
\end{aligned}
$$

Fazendo a mudança

$$
x=\sqrt{\frac{1-\tau^{2}}{\tau}} u
$$

obtemos

$$
1=\left\langle e^{\sqrt{\frac{1-\tau^{2}}{\tau}}(\bar{u} z+u \bar{z})-\frac{1}{\tau} u \bar{u}+\frac{1}{2 \tau}\left(u^{2}+\bar{u}^{2}\right)}\right\rangle
$$

ou equivalentemente

$$
e^{\frac{1}{\tau} u \bar{u}}=\left\langle e^{\sqrt{\frac{1-\tau^{2}}{\tau}} \bar{u} z+\frac{1}{2} \bar{u}^{2}} e^{\sqrt{\frac{1-\tau^{2}}{\tau}} u \bar{z}+\frac{1}{2} u^{2}}\right\rangle .
$$


Notamos que o lado direito da expressão anterior envolve funções geratrizes para os polinômios de Hermite mônicos (E.3). Assim, expandindo a expressão acima obtemos

$$
\sum_{j=0}^{\infty} \frac{1}{j !}\left(\frac{u \bar{u}}{\tau}\right)^{j}=\sum_{k, l=0}^{\infty} \frac{\bar{u}^{l} u^{k}}{l ! k !}\left\langle H_{k+1}\left(\sqrt{\frac{1-\tau^{2}}{\tau}} \bar{z}\right) H_{l+1}\left(\sqrt{\frac{1-\tau^{2}}{\tau} z}\right)\right\rangle
$$

e pela definição de média (E.6) e da equação (E.5) temos

$$
\int \frac{d^{2} z}{\pi} e^{-\frac{1}{2} Z^{*} A Z} H_{k+1}\left(\sqrt{\frac{1-\tau^{2}}{\tau}} \bar{z}\right) H_{l+1}\left(\sqrt{\frac{1-\tau^{2}}{\tau} z}\right)=\frac{l ! \delta_{k, l}}{\tau^{l} \sqrt{1-\tau^{2}}}
$$

para todo $k, l \in \mathbb{N}$. Escalando $z \operatorname{com} N$ da forma

$$
z \longrightarrow \sqrt{\frac{N}{1-\tau^{2}} z}
$$

temos

$$
e^{-\frac{1}{2} Z^{*} A Z} d^{2} z \longrightarrow \frac{N}{1-\tau^{2}} e^{-N V_{\tau}(z)} d^{2} z
$$

por (E.4), (E.2) e (E.1). Logo,

$$
\int H_{k+1}\left(\sqrt{\frac{N}{\tau}} \bar{z}\right) H_{l+1}\left(\sqrt{\frac{N}{\tau} z}\right) \frac{N}{1-\tau^{2}} e^{-N V_{\tau}(z)} \frac{d^{2} z}{\pi}=\frac{l ! \delta_{k, l}}{\tau^{l} \sqrt{1-\tau^{2}}}
$$

e, portanto,

$$
\phi_{j+1}^{G}(z)=\frac{\tau^{\frac{j}{2}} \sqrt{N}}{\sqrt{j ! \pi}\left(1-\tau^{2}\right)^{\frac{1}{4}}} H_{j+1}\left(\sqrt{\frac{N}{\tau} z}\right)
$$

é tal que

$$
\int \overline{\phi_{j}^{G}(z)} \phi_{k}^{G}(z) e^{-N V_{\tau}(z)} d^{2} z=\delta_{k, l}, k, l \in \mathbb{N}^{+}
$$

como queríamos.

Observação E.2 Podemos encontrar esta prova em [40].

Teorema E.3 Sejam $\left\{\phi_{j+1}^{G}\right\}_{j=1}^{N}$ os polinômios ortonormais

$$
\phi_{j+1}^{G}(z)=\frac{\tau^{\frac{j}{2}} \sqrt{N}}{\sqrt{j ! \pi}\left(1-\tau^{2}\right)^{\frac{1}{4}}} H_{j+1}\left(\sqrt{\frac{N}{\tau}} z\right)
$$

com respeito ao peso

$$
d \nu(z)=e^{-N V_{\tau}(z)} d^{2} z
$$

e $V_{\tau}: \mathbb{C} \rightarrow \mathbb{R}$ o potencial dado por (E.1). Então

$$
K_{N}^{G}(z, w)=\frac{N}{\pi \sqrt{\left(1-\tau^{2}\right)}} e^{-\frac{N}{2} V_{\tau}(z)} e^{-\frac{N}{2} \overline{V_{\tau}(w)}} \sum_{j=0}^{N-1} \frac{\tau^{j}}{j !} H_{j+1}\left(\sqrt{\frac{N}{\tau} z}\right) H_{j+1}\left(\sqrt{\frac{N}{\tau}} \bar{w}\right) .
$$


Prova. Com auxílio do Lema anterior e da definição do núcleo integral temos

$$
\begin{aligned}
K_{N}^{G}(z, w) & =e^{-\frac{N}{2} V_{\tau}(z)} e^{-\frac{N}{2} \overline{V_{\tau}(w)}} \sum_{j=1}^{N} \phi_{j}^{G}(z) \overline{\phi_{j}^{G}(w)} \\
& =e^{-\frac{N}{2} V_{\tau}(z)} e^{-\frac{N}{2} \overline{V_{\tau}(w)}} \sum_{j=0}^{N-1} \frac{\tau^{j} N}{j ! \pi \sqrt{\left(1-\tau^{2}\right)}} H_{j+1}\left(\sqrt{\left.\frac{N}{\tau} z\right) H_{j+1}\left(\sqrt{\frac{N}{\tau}} \bar{w}\right)}\right. \\
& =\frac{N}{\pi \sqrt{\left(1-\tau^{2}\right)}} e^{-\frac{N}{2} V_{\tau}(z)} e^{-\frac{N}{2} \overline{V_{\tau}(w)}} \sum_{j=0}^{N-1} \frac{\tau^{j}}{j !} H_{j+1}\left(\sqrt { \frac { N } { \tau } z ) H _ { j + 1 } } \left(\sqrt{\frac{N}{\tau}} \bar{w} .\right.\right.
\end{aligned}
$$

como queríamos.

\section{E.1 Comportamento assintótico.}

Lema E.4 (Mehler) Seja $H_{j}$ o j-ésimo polinômio mônico de Hermite

$$
e^{x \tau-\frac{\tau^{2}}{2}}=\sum_{j=0}^{\infty} H_{j+1}(x) \frac{\tau^{j}}{j !}
$$

e $H_{j}(z)\left(H_{j}(\bar{z})\right)$ para $z \in \mathbb{C}$ a extensão analítica (anti-analítica) de $H_{j}(x)$ para $x \in \mathbb{R}$. Então

$$
\sum_{j=0}^{\infty} \frac{\tau^{j}}{j !} H_{j+1}(z) H_{j+1}(\bar{w})=\frac{1}{\sqrt{1-\tau^{2}}} e^{\frac{2 z \bar{w} \tau-\tau^{2}\left(z^{2}+\bar{w}^{2}\right)}{2\left(1-\tau^{2}\right)}}
$$

Prova. O Lema segue se provarmos que

$$
\sum_{j=0}^{\infty} \frac{\tau^{j}}{j !} H_{j+1}(x) H_{j+1}(y)=\frac{1}{\sqrt{1-\tau^{2}}} e^{\frac{2 x y \tau-\tau^{2}\left(x^{2}+y^{2}\right)}{2\left(1-\tau^{2}\right)}}
$$

pois a partir desta identidade podemos estender $H_{j}$ de maneira conveniente. Da identidade

$$
\int_{-\infty}^{\infty} e^{-a u^{2}+b u} d u=\sqrt{\frac{\pi}{a}} e^{\frac{b^{2}}{4 a}}
$$

para $a, b \in \mathbb{C}$ com $\operatorname{Re}(a)>0$ obtemos a expressão

$$
e^{-\frac{x^{2}}{2}}=\frac{1}{\sqrt{2 \pi}} \int_{-\infty}^{\infty} e^{-\frac{1}{2} u^{2}+i x u} d u
$$

e da fórmula de Rodrigues

$$
H_{j+1}(x)=(-1)^{j} e^{\frac{x^{2}}{2}} \frac{d^{j}}{d x^{j}} e^{-\frac{x^{2}}{2}}
$$


obtemos

$$
H_{j+1}(x)=\frac{e^{\frac{x^{2}}{2}}}{\sqrt{2 \pi}} \int_{-\infty}^{\infty}(-i u)^{j} e^{-\frac{1}{2} u^{2}+i x u} d u
$$

e, portanto,

$$
\begin{aligned}
& \sum_{j=0}^{\infty} \frac{\tau^{j}}{j !} H_{j+1}(x) H_{j+1}(y) \\
& =\sum_{j=0}^{\infty} \frac{e^{\frac{x^{2}+y^{2}}{2}}}{2 \pi} \int_{-\infty}^{\infty} \int_{-\infty}^{\infty} \frac{(-u v \tau)^{j}}{j !} e^{-\frac{1}{2} u^{2}+i x u} e^{-\frac{1}{2} v^{2}+i y v} d v d u \\
& =\frac{e^{\frac{x^{2}+y^{2}}{2}}}{2 \pi} \int_{-\infty}^{\infty} \int_{-\infty}^{\infty} \sum_{j=0}^{\infty} \frac{(-u v \tau)^{j}}{j !} e^{-\frac{1}{2} u^{2}+i x u} e^{-\frac{1}{2} v^{2}+i y v} d v d u \\
& =\frac{e^{\frac{x^{2}+y^{2}}{2}}}{2 \pi} \int_{-\infty}^{\infty} \int_{-\infty}^{\infty} e^{-u v \tau} e^{-\frac{1}{2} u^{2}+i x u} e^{-\frac{1}{2} v^{2}+i y v} d v d u \\
& =\frac{e^{\frac{x^{2}+y^{2}}{2}}}{2 \pi} \int_{-\infty}^{\infty} e^{-\frac{1}{2} u^{2}+i x u} \int_{-\infty}^{\infty} e^{-\frac{1}{2} v^{2}+(i y-u \tau) v} d v d u \\
& =\frac{e^{\frac{x^{2}+y^{2}}{2}}}{2 \pi} \int_{-\infty}^{\infty} e^{-\frac{1}{2} u^{2}+i x u} \sqrt{2 \pi} e^{\frac{1}{2}(i y-u \tau)^{2}} d u \\
& =\frac{e^{\frac{x^{2}}{2}}}{\sqrt{2 \pi}} \int_{-\infty}^{\infty} e^{-\frac{1-\tau^{2}}{2} u^{2}+i(x-y \tau) u} d u \\
& =\frac{e^{\frac{x^{2}}{2}}}{\sqrt{2 \pi}} \sqrt{\frac{\pi}{\frac{1-\tau^{2}}{2}}} e^{\frac{(i(x-y \tau))^{2}}{4\left(\frac{1-\tau^{2}}{2}\right)}} \\
& =\frac{1}{\sqrt{1-\tau^{2}}} e^{\frac{x^{2}}{2}-\frac{x^{2}-2 \tau x y+\tau^{2} y^{2}}{2\left(1-\tau^{2}\right)}} \\
& =\frac{1}{\sqrt{1-\tau^{2}}} e^{\frac{1}{2\left(1-\tau^{2}\right)}\left(2 \tau x y-\tau^{2}\left(x^{2}+y^{2}\right)\right)}
\end{aligned}
$$

como queríamos. Notamos que a troca da soma com a integral é lícita, pois todas as somas e integrais usadas acima são absolutamente convergentes e nas integrações foi usado a identidade (E.8).

Observação E.5 Esta prova de (E.7) é atribuída a G. H. Hardy por G. N. Watson em [41]. 
Teorema E.6 Sejam $\left\{\phi_{j}^{G}\right\}_{j=1}^{N}$ os polinômios ortonormais com respeito ao peso

$$
d \nu^{G}(z)=e^{-N V_{\tau}(z)} d^{2} z
$$

e $V_{\tau}: \mathbb{C} \rightarrow \mathbb{R}$ o potencial dado por (E.1) (notamos que este tipo de potencial satisfaz o Teorema 40, pois $\tau \in] 0,1[$ ). Então a expansão assintótica em $N$ para o núcleo integral associado a este problema é dada por

$$
K_{N}^{G}(z, w)=\frac{N}{\pi\left(1-\tau^{2}\right)} e^{\frac{N}{1-\tau^{2}}\left(z \bar{w}-\frac{z \bar{z}}{2}-\frac{w \bar{w}}{2}+\frac{\tau}{4}\left(\bar{z}^{2}-z^{2}+w^{2}-\bar{w}^{2}\right)\right)}(1+o(1))
$$

Prova. Do Teorema E.3 e do Lema E.4 temos

$$
\begin{aligned}
K_{N}^{G}(z, w) & =\frac{N}{\pi\left(1-\tau^{2}\right)} e^{-\frac{N}{2} V_{\tau}(z)} e^{-\frac{N}{2} \overline{V_{\tau}(w)}} e^{\frac{1}{2\left(1-\tau^{2}\right)} \frac{N}{\tau}\left(2 \tau z \bar{w}-\tau^{2}\left(z^{2}+\bar{w}^{2}\right)\right)}(1+o(1)) \\
& =\frac{N}{\pi\left(1-\tau^{2}\right)} e^{\frac{N}{1-\tau^{2}}\left(z \bar{w}-\frac{z \bar{z}}{2}-\frac{w \bar{w}}{2}+\frac{\tau}{4}\left(\bar{z}^{2}-z^{2}+w^{2}-\bar{w}^{2}\right)\right)}(1+o(1))
\end{aligned}
$$

como queríamos. Na última passagem foi usado (E.1).

Observação E.7 Este resultado pode ser encontrado em [42].

Observação E.8 Notamos que

$$
\lim _{N \rightarrow \infty} \frac{1}{N} K_{N}^{G}(z, z) d^{2} z=\frac{1}{\pi\left(1-\tau^{2}\right)} d^{2} z=\rho^{V_{\tau}}(z) d^{2} z=d \mu^{V_{\tau}}
$$

onde $\mu^{V_{\tau}}$ é a medida de equilíbrio associada ao potencial de Ginibre-Girko $V_{\tau}$. 


\section{Bibliografia}

[1] E. P. Wigner. "Distribution laws for the roots of a random Hermitian matrix" em "Statistical theories of spectra: fluctuations" de C. E. Porter. Academic Press (1965).

[2] C. E. Porter (ed.): "Statistical Theories of Spectra: Fluctuations", Academic Press (1965).

[3] F. J. Dyson. "Statistical Theory of the Levels of Complex Systems I". Jour. of Math. Phys. 3, 140-156, (1962).

[4] F. J. Dyson. "Statistical Theory of the Levels of Complex Systems III". Jour. of Math. Phys. 3, 166-175, (1962).

[5] D. L. Mehta. "Random Matrices - Revised and Enlarged". 2 ${ }^{\text {nd }}$ Edition. Academic Press. 1991.

[6] P. J. Forrester, N. C. Snaith, J. J. M. Verbaarschot. "Developments in random matrix theory". J. Phys. A: Math. Gen. 36 (2003) R1-R10.

[7] P. Deift. "Orthogonal Polynomials and Random Matrices: A Riemann-Hilbert Approach". American Mathematical Society. Courant Institute of Mathematical Sciences, New York University, New York. Lecture Notes 3, 2000.

[8] J. Ginibre. "Statistical Ensembles of Complex, Quaternion and Real Matrices". Joun. of Math. Phys. 6, Issue 3, pp. 440-449, (1965). Pac. Jour. Math. 193 (2000) no 2 355369.

[9] A. B. de Monvel, L. Pautur e M. Shcherbina. "On the Statistical Mechanics Approach in the Random Matrix Theory: Integrated Density of States". Jour. of Stat. Phys. 79, 585-611 (1995).

[10] B. Rider. "A limit theorem at the edge of a non-Hermitian random matrix ensemble". J. Phys. A: Math. Gen. 36 3401-3409 (2003). 
[11] Z. D. Bai. "Circular Law". The Ann. of Prob. 25 no 1 494-529, (1997).

[12] Z. D.Bai. "Methodologies in spectral analysis of large dimensional random matrices: a review". Statist. Sinica 9 611-677 (1999).

[13] P. Lancaster and M. Tismenetsky. Theory of Matrices, $2^{\text {nd }}$ edition with aplications. Academic Press, San Diego, (1985).

[14] M. Kiessling H. Spohn. "A note on the eigenvalue density of random matrices". Comm. Math. Phys. 199 (1999), no 3, 683-695.

[15] P. Leboeuf. "Random matrices, random polynomials and Coulomb systems". Proceedings of the International Conference on Strongly Coupled Coulomb Systems, Saint-Malo, 1999 (EDP Science, Les Ulis). http://arxiv.org/PS_cache/condmat/pdf/9911/9911222.pdf.

[16] D. Ruelle. "Statistical Mechanics: Rigorous Results". Addison-Wesley Publishing Company, 1989.

[17] P. B. Wiegmann, A. Zabrodin. "Conformal Maps and Integrable Hierarchies". Comm. Math. Phys. 213, 523-538 (2000).

[18] P. Elbau; G. Felder. "Density of eigenvalues of random normal matrices". Comm. Math. Phys. 259, no 2, 433-450 (2005).

[19] Y. V. Fyodorov, B. A. Khoruzhenko, H.-J. Sommer. "Almost Hermitian Random Matrices: Crossover from Wigner-Dyson to Ginibre Eigenvalue Statistics". Phys. Rev. Lett. 79(4), 557-560, (1997).

[20] P. Deift, T. Kriecherbauer, K. T.-R. McLaughlin, S. Venekides e X. Zhou: Commun. Pure Appl. Math. 52, 1335-1425 (1999).

[21] G. Oas. "Normal Matrix Models". Tese de doutorado, Universidade da Califórmia, Davis. 1995.

[22] G. Oas. "Universal cubic eigenvalue repulsion for random normal matrices". Phys, Rev. E 55, 205 (1997).

[23] F. Haake. "Quantum Signatures og Chaos", Springer-Verlag, Berlin, 1991. 
[24] E. B. Saff e V. Totik. "Logarithmic potentials with external fields". Spring, Nes York-Berlin, (1997).

[25] W. Rudin. "Real and Complex Analysis". McGraw-Hill, New York, 2nd edition, 1974.

[26] R. E. Greene. "Function Theory of One Complex Variable". 2nd ed. Graduate studies in Mathematics, ISSN 1065-7339; v. 40, 2001.

[27] H. Hedenmalm, N. Makarov. "Quantum Hele-Shaw flow". arXiv: math. PR/0411437 v1 19/11/2004.

[28] P. J. Davis. "The Schwarz functions and its Applications". The Carus Mathematical Monographs, $\mathrm{n}^{\circ}$ 17. The mathematical association of America.

[29] A. Soshnikov. "Determinantal random point fields". Russian Math. Surveys 55:5 923-975 (2000).

[30] M. Abramovitz e I. A. Stegun. "Handbook of Mathematical Functions". Dover, New York, 1970 .

[31] M. Born, E. Wolf. "Principles of optics". Pergamon press. 1959.

[32] D. G. Figueiredo. "Análise de fourier e equações diferenciais parciais". IMPA - CNPq, 1977.

[33] W. Rudin. "Principles of mathematical analysis". McGraw-Hill, 1964.

[34] J. D. Murray. "Asymptotic analysis". Clarendon Press - Oxford, 1974.

[35] M. Englis. "Asymptotic Behaviour og reproducing kernels og weighted Bergman spaces". Trans. Amer. Math. Soc. 349, 9, 3717-3735, (1997).

[36] G. A. Korn, T. M. Korn. "Mathematical Handbook for Scientists and Engineers: Definitions, Theorems and Formulas for Reference and Review", Dover Publications, INC. 2000.

[37] C. A. Berenstein, R. Gay. "Complex Variables, an Introduction". Graduate Texts in Mathematics. Springer, 1991.

[38] B. Dubrovin, S. Novikov, A. Fomenko. "Géométrie contemporaine. Methodes et application". Parte I seg. ed. editora FS. 
[39] R. Courant, D. Hilbert. "Methods of Mathematical Physics". Vol 1. Interscience Publishers, Inc. New York, 1953.

[40] F. di Francesco, M. Gaudin, C. Itzykson, F.Lesage. "Laughlin wave-funtions, Coulomb gases and expansions of the discriminant", Internat. J. Modern Phys. A 9 (1994), 4257-4351.

[41] G. N. Watson. "Notes on generating functions of polynomials: Hermite polynomials". Journal of the London Mathematical Society 8 (1933), 194-199.

[42] Y. V. Fyodorov, H.-J. Sommers, B. A. Khoruzhenko. "Universality in the random matrix spectra in the regime of weak non-Hermiticity". Annales de l' I. H. Poincaré, sec. A, t. $68, \mathrm{n}^{\circ} 2$ (1998), p. 449-489. 